TOPICAL REVIEW

\title{
Violation of the fluctuation-dissipation theorem in glassy systems: basic notions and the numerical evidence
}

\author{
A Crisanti†and F Ritort \\ † Dipartimento di Fisica, Università di Roma "La Sapienza", INFM Sezione di \\ Roma I and SMC, P.le Aldo Moro 2, 00185 Roma, Italy \\ $\ddagger$ Departament de Física Fonamental, Facultat de Física, Universitat de \\ Barcelona, Diagonal 647, 08028 Barcelona, Spain \\ E-mail: andrea.crisanti@phys.uniroma1.it, ritort@ffn.ub.es
}

\begin{abstract}
This review reports on the research done during the past years on violations of the fluctuation-dissipation theorem (FDT) in glassy systems. It is focused on the existence of a quasi-fluctuation-dissipation theorem (QFDT) in glassy systems and the currently supporting knowledge gained from numerical simulation studies. It covers a broad range of non-stationary aging and stationary driven systems such as structural-glasses, spin-glasses, coarsening systems, ferromagnetic models at criticality, trap models, models with entropy barriers, kinetically constrained models, sheared systems and granular media. The review is divided into four main parts: 1) An introductory section explaining basic notions related to the existence of the FDT in equilibrium and its possible extension to the glassy regime (QFDT), 2) A description of the basic analytical tools and results derived in the framework of some exactly solvable models, 3) A detailed report of the current evidence in favour of the QFDT and 4) A brief digression on the experimental evidence in its favour. This review is intended for inexpert readers who want to learn about the basic notions and concepts related to the existence of the QFDT as well as for the more expert readers who may be interested in more specific results.
\end{abstract}

PACS numbers: 64.40.-i, 64.60.Cn, 75.10.Nr 


\section{Contents}

1 Introduction 3

$\begin{array}{lll}2 & \text { Basic definitions and concepts } & 6\end{array}$

2.1 The microcanonical and canonical ensembles . . . . . . . . . . 6

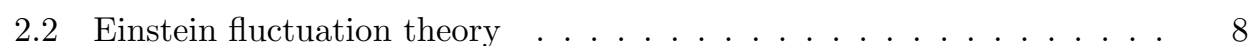

2.3 The Onsager regression principle: a simple derivation of the fluctuation-dissipation theorem (FDT) 9

$3 \quad$ The Master Equation Approach 12

3.1 The Master Equation (ME) . . . . . . . . . . . . . . . . . . . . 12

3.2 Correlations responses and the FDT . . . . . . . . . . . . . . 14

3.3 The Component Master Equation . . . . . . . . . . . . . . . . . . 18

$4 \quad$ FDT extensions to the non-equilibrium regime $\quad 19$

4.1 An intermezzo on aging . . . . . . . . . . . . . . . 19

4.2 The unbiased component ensemble and the master free energv equation 22

4.2.1 Complexity and the effective temperature ......... 24

4.3 The integrated response function (IRF) and fluctuation-dissipation (FD) plots 25

4.4 The concept of neutral observables . . . . . . . . . . . . . . . . 28

4.5 Numerical approach to component dvnamics: Stillinger-Weber decomposition 29

$5 \quad$ Thermodvnamic description of the aging state 32

5.1 Methods to compute the complexity . . . . . . . . . . . . . 32

5.1 .1 Analvtical methods. . . . . . . . . . . . . . . . . 32

5.1 .2 Numerical methods. . . . . . . . . . . . . . . . . . . . 34

5.2 The concept of the effective temperature . . . . . . . . . . . . . . . 37

5.3 The Edwards' measure for granular materials . . . . . . . . . . . . . 41

6 QFDT from exactly solvable models 43

6.1 The Mode-Coupling Theorv . . . . . . . . . . . . . . . . . . . . . 43

6.2 Disordered spin-glass models . . . . . . . . . . . . . . . . . . . . . 45

$6.2 .1 \quad p$-spin spherical model. . . . . . . . . . . . . . . . 45

6.2 .2 The Sherrington-Kirkpatrick model. . . . . . . . . . . . . . 51

6.3 Random manifolds and diffusive models . . . . . . . . . . . . . . . 54

6.4 Trap models . . . . . . . . . . . . . . . . . . . . . . . . 57

6.5 Models with entropv barriers . . . . . . . . . . . . . . . 60

6.5 .1 Oscillator models. . . . . . . . . . . . . . . . . 61

6.5.2 The Backsammon and urn models . . . . . . . . . . . 66

6.6 Ferromagnetic models at criticalitv . . . . . . . . . . . . . . . 67

$\begin{array}{lll}7 & \text { QFDT: the numerical evidence } & 71\end{array}$

7.1 Structural glasses . . . . . . . . . . . . . . . . . . . . . . . . . 71

7.1.1 Mixtures of soft particles of different sizes . . . . . . . . . 71

7.1 .2 Lennard-Jones binarv-mixtures . . . . . . . . . . . . . . 72

7.1 .3 Monoatomic Lennard-Jones svstems . . . . . . . . . . . . . 73

$7.1 .4 \quad$ Finite-size mean-field glasses $\ldots \ldots \ldots$. . . . . . . . . 75

7.2 Spin glasses and other random svstems . . . . . . . . . . . . . . $\quad 78$

$7.2 .1 \quad$ Spin glasses . . . . . . . . . . . . . . . . . . . . . . . . 80

7.2 .2 Other random svstems . . . . . . . . . . . . . . . 86 
7.3 Coarsening svstems . . . . . . . . . . . . . . . . . . 87

7.4 Non-relaxational driven svstems . . . . . . . . . . . . . . . . . . . . . . 92

7.4.1 Sheared svstems . . . . . . . . . . . . . . . . . . . . . . . . . . . . . . 92

7.4 .2 Tapped svstems . . . . . . . . . . . . . . . . . . . 96

7.5 Kinetically constrained models . . . . . . . . . . . . . . . . . . . 101

8 QFDT: the experimental evidence 104

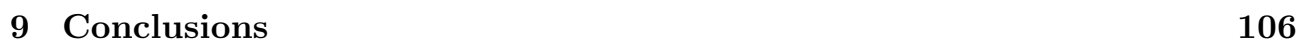

$\begin{array}{ll}10 \text { List of abbreviations } & 108\end{array}$

\section{Introduction}

The search for a general theory of non-equilibrium processes has been a primary goal in modern statistical physics. Despite of many efforts in this direction we have a limited understanding of the basic principles behind non-equilibrium theories. Compared with ensemble equilibrium theory, a general principle like the equal probability Boltzmann principle (that forms the basis of equilibrium statistical mechanics and provides a statistical foundation of thermodynamics) is still lacking. During the last century, the field of non-equilibrium phenomena has grown in two directions: 1) by developing new statistical models as an inspiring source of fruitful new concepts and ideas 2) by establishing partial links among different, apparently disconnected, non-equilibrium phenomena.

Although much progress has been made in the first direction, the second one remains less unexplored. While a general principle governing non-equilibrium systems probably does not exist, substantial progress could be done following the second route in the search for basic principles governing a restricted category or class of systems. The applications of such basic principles may be very important because a priori many different systems can fall into the same category. Hence the interest in the research on the existence of such restricted formulations.

During the past years it has become increasingly clear that glassy systems may constitute one of these large categories where their physical behavior can be rationalized within a restricted formulation. Glassy systems are rather common in nature and many systems such as structural glasses, spin glasses, disordered and granular materials or proteins present what is called glassy behaviors. This means a dramatic slowing down of relaxational processes when some control parameters are varied. A typical signature of glassy behaviour is a power-law or stretched exponential behavior of correlation functions, as opposed to exponential decay. As the characteristic relaxation time may change by several orders of magnitude it can easily exceed the observation time. As a consequence the system ages: the observed static and dynamic properties depend on the age of the system defined as the time since the system was prepared (also called waiting time). For this reason this residual very slow non-equilibrium phenomena is commonly known as aging.

Aging systems include a large variety of materials. In fact, nearly all physical systems, within an appropriate set of conditions and observed during a specific timewindow, display glassy properties. The origin of glassy behaviour, however, can vary from system to system. The most important class of glassy systems (which include window glasses) are glass forming liquids where glassy behaviour is due to 
the appearance, as some external parameter is changed, of a long-lived complex pattern of interacting bonds between their microscopic constituents which strongly inhibits relaxation toward equilibrium. Aging follows from the very-slow motion of such a complex pattern of interacting bonds which induces a slow change of the atomic structure of the liquid. For this reason glass-forming liquids are usually called structural glasses. Our current understanding of the slow glassy relaxation dynamics is greatly limited by the lack of a general non-equilibrium theory that accounts for these phenomena.

Glasses can be generated by the fast cooling of a liquid. Upon cooling from high temperatures down to the melting transition temperature $T_{\mathrm{M}}$, sometimes crystallization does not occur and the liquid continues its way down in temperature beyond $T_{\mathrm{M}}$ by following a line (called supercooled liquid line) which is the continuation of the liquid line. As the liquid line is thermodynamically stable only above $T_{\mathrm{M}}$, the supercooled liquid line is metastable with locally equilibrated properties, so its lifetime can be extremely large. As cooling proceeds it is observed that the supercooled liquid falls out of equilibrium (i.e. departs from the supercooled liquid line), below a temperature $T^{*}(r)$ which depends on the cooling rate $r$. The state reached below $T^{*}(r)$ is called a glass and the corresponding relaxational regime is indistinctly termed as aging or glassy. For small values of $r$ a sharp transition is observed at $T^{*}(r)$, usually referred as structural arrest, where the heat capacity jumps down, indicating the freezing of degrees of freedom. Contrarily to the supercooled state, the glass state is of non-equilibrium nature and $T^{*}(r)$ is observed to decrease with $r$. As $T^{*}(r)$ depends on the cooling rate, no equilibrium phase transition occurs at that temperature. This means that the liquid will eventually equilibrate back to the supercooled state. The equilibration process may take an extremely long time (even for temperatures only a few degrees below $T^{*}$ ) being inaccessible from any practical point of view. Under some conditions the equilibration time can be larger than the age of the universe!. In these conditions the glass state is the only observable state.

Long equilibration times imply that the glass state is characterized by very low energy dissipation rates, also called entropy production. This may give the false impression that the glass is in a stationary state. For instance, a piece of silica glass at room temperature looks pretty stable, indeed its optical, electrical and mechanical properties appear constant in time. However a more careful examination reveals that the physical properties are constant only if observed on time-scales much smaller than the time elapsed since the glass was prepared or formed. Beyond that timescale, the physical properties change revealing that the glass is aging.

Although aging was identified long time ago in the study polymers [1 it has received a renewed interest in connection with the study of spin glasses. Measurements of the magnetization in spin glasses have shown that aging is a general property of the low-temperature spin-glass phase. There are several types of spin glass materials, the most common ones are metallic spin glasses. These are random diluted magnetic systems where glassy behaviour arises from the disordered pattern of exchange interactions, rather than being self-generated as in structural glasses. Indeed random dilution generates exchange interactions with random competing signs, the system is then frustrated since a finite fraction of bonds cannot be satisfied. Aging is consequence of the slow evolution of the pattern of satisfied bonds which becomes strongly inhibited as the temperature is lowered.

Another class of systems with glassy properties are driven systems which, under certain conditions, reach a stationary state characterized by non-Gibbsian probability 
distributions. After applying a time-dependent perturbation of frequency $\omega>1 / t_{\text {eq }}$, upon an initially equilibrated system of relaxation time $t_{\text {eq }}$, a new stationary state is reached which for many aspects is similar to the aging state of the relaxational system of age $\sim 1 / \omega$.

Another important aspect of glassy systems that has received considerable attention for a long time [2] is the idea about the existence of an effective temperature (sometimes also called fictive temperature) describing the non-equilibrium properties of the glassy state. During the last decades it has emerged that a possible way to rationalize the existence of an effective temperature is by measuring violations of the fluctuation-dissipation theorem (FDT). In glassy systems, a new modified relation between correlations and responses that goes under the name of quasi FDT (QFDT) provides a description of the dynamics in the glassy state by quantifying the violations of the FDT. In this new theorem the effective temperature plays the role of the temperature of the bath. Related to the concept of the effective temperature is the idea of the existence of a heat flow from the glass to the thermal bath put in contact with the system. As the glass has an effective temperature larger than that of the bath, the heat flows from the glass to the bath. However, the energy dissipation rate from the glass toward the bath is extremely low (hardly measurable) and, in general, this flow can be also understood in terms of an effective very low thermal conductivity. The reader should be aware that using QFDT is only one among other possible ways of introducing an effective temperature for the description of the glassy state. In general other definitions which use a generalization of different equilibrium relations to the non-equilibrium regime are possible. This arises the problem of the equivalence of all possible definitions. We shall not discuss this point in this review and we will stick to the QFDT definition of an effective temperature.

This review will concentrate on the existence of a QFDT, its physical meaning, in what conditions it can emerge and the numerical evidence reported in favour of its validity. This is a rapidly growing area of research which is attracting new condensed matter and statistical physicists. We will report here the most important results obtained until the summer of the year 2002. Although we have tried to cover most of the published work some contributions may have been overlooked. We apologize in advance to those colleagues. Although some of the results here reviewed are currently well understood many others still lack a full comprehension so it is not exaggerated to say that some of the ideas and suggestions described in this review could be modified in the future to adapt to the forthcoming theoretical, numerical or experimental evidence. Most of the results here reported deal with relaxational aging systems (as compared to driven systems) since these are the ones that have mostly attracted the attention of the researchers in the field. However, future developments in this exciting area of research might compensate this original unbalance as driven systems appear more amenable to experimental research than aging systems. Moreover, in this review we shall only consider the FDT in its classical version. Although most of the the ideas can be extended to the quantum regime, to our knowledge there are neither numerical or experimental works challenging FDT violations in the quantum aging regime. Therefore we shall not address them, the interested reader is referred to a recent review [3].

Many textbooks and article reviews can be useful to complement the contents of this review. Basic reviews on the glass transition since mid 80's until now can be found in [4, 5, 6, 7]. Other accounts dealing with aspects of the glass transition include: thermodynamic theories of the glass transition [8, 9, mode-coupling theory (MCT) 
[10, 11] and numerical simulations [12]. For spin glasses a good selection of review articles can be found in 13. A clear discussion of mode-coupling approximations in the context of disordered systems can be found in [14. A recent discussion of several aspects of concerning FDT violations can be found also in a recent review on kinetically constrained models 15. Finally, a throughout compendium of analytical methods for glassy dynamics has been recently collected in [3] and a review of granular systems in [16. Proceeding articles covering several aspects of glasses and spin-glasses can be found in [17, 18] and for kinetically constrained models in [19].

The contents of this review have been written with two kinds of readers in mind: inexpert and expert. Those inexpert readers who want to understand the most basic ideas as well as the interest of investigating FDT violations must read Sections 2 314 These sections have been written at an introductory level, so expert readers who know about the subject may directly start reading from Section 5 However, a careful reading of section 4 is recommended to those readers who want to have a more physically appealing description of the possible origin of FDT violations. Section 5 deals with some of the thermodynamic consequences of FDT violations. Sections 617 constitute the core of the review. Section 6 describes our knowledge of FDT violations gathered from several exactly solvable models where many aspects of their non-equilibrium behavior can be understood by analytical means. Section[7 covers all evidence collected in the past years in favour of the existence of a QFDT in glassy systems. Many of the model systems described in this section correspond to realistic as well as model systems for which analytical solutions are hardly known. The expert reader who wants to grasp the state of the art concerning these questions will be mainly interested on these two sections. Finally, a brief account of some experimental results on FDT violations is described in Section 8 Section 9 presents some conclusions.

\section{Basic definitions and concepts}

In this section we recall some concepts of equilibrium theory which will be needed later for the description the glassy state.

\subsection{The microcanonical and canonical ensembles}

The foundations of equilibrium statistical mechanics rely on the maximum entropy postulate and the Boltzmann's equal probability hypothesis. An introduction to the basic postulates can be found in the classical books on statistical mechanics, rather excellent are that by $\mathrm{Ma}$ [20] and Callen [21]. Good discussions also comes from the Information Theory, see for example the book of Beck and Schloegel [22].

In what follows we shall denote with $\mathcal{C}$ a generic system configuration in the phase space. The phase space can be either continuous or discrete depending on the particular system. For example, for a system of $N$ particles $\mathcal{C}$ are the positions and momenta in a continuous $6 N$-dimensional space, while for a system of $N 1 / 2$-spins $\mathcal{C}$ is a point in a a discrete $N$ dimensional space with $2^{N}$ points. The system evolves in time following a dynamical rule which generally speaking is a rule that for each configuration $\mathcal{C}$ associates a new configuration $\mathcal{C}^{\prime}$. The set of configurations which can be visited given a dynamical rule, defines the region of motion in the phase space. Let $\Gamma$ be the volume of the region of motion allowed by the invariant quantities. The basic assumption of statistical mechanics asserts that the entropy is the logarithm of $\Gamma$. This makes the entropy computable without having to solve the dynamics. If we 
assume that the system is described by an energy function $E(\mathcal{C})$ then the motion is confined to a region in phase space of constant energy. The calculation of entropy is then reduced to

$$
S(E)=\ln \sum_{\mathcal{C}} \delta(E-E(\mathcal{C}))
$$

where in the case of continuous variables the sum must be read as an integral. This equation defines the microcanonical ensemble. Since all allowed states are included $S(E)$ is clearly a maximum over all possible regions of constant energy $E$ into which the phase space can be divided.

Let us consider now an observable $A(\mathcal{C})$ which we will assume to be neither a constant of motion or a univocal function of the energy $E$. We also assume that $A(\mathcal{C})$ is extensive, i.e., it is proportional to the system size (volume or the number of constituents). We can then divide the phase space according to the value of $A(\mathcal{C})$ and, defining the degeneration $\Omega(E, A)$ of the partition as the total number of configurations $\mathcal{C}$ of energy $E$ and observable value $A$ :

$$
\Omega(E, A)=\sum_{\mathcal{C}} \delta(E-E(\mathcal{C})) \delta(A-A(\mathcal{C})),
$$

introduce the entropy in analogy with (10):

$$
S(E, A)=\ln \Omega(E, A) .
$$

Using the integral representation of delta functions $\Omega(E, A)$ can be rewritten as

$$
\begin{gathered}
\Omega(E, A)=\int_{-\infty}^{\infty} \frac{d \alpha_{1} d \alpha_{2}}{(2 \pi)^{2}} \exp \left(i \alpha_{1} E+i \alpha_{2} A\right) \sum_{\mathcal{C}} \exp \left[-i \alpha_{1} E(C)-i \alpha_{2} A(C)\right] \\
=\int_{-\infty}^{\infty} \frac{d \alpha_{1} d \alpha_{2}}{(2 \pi)^{2}} \exp \left[\mathcal{S}\left(E, A, \alpha_{1}, \alpha_{2}\right)\right]
\end{gathered}
$$

where the function $\mathcal{S}\left(E, A, \alpha_{1}, \alpha_{2}\right)$ is given by,

$$
\mathcal{S}\left(E, A, \alpha_{1}, \alpha_{2}\right)=i \alpha_{1} E+i \alpha_{2} A+\ln \mathcal{Z}\left(\alpha_{1}, \alpha_{2}\right)
$$

and $\mathcal{Z}\left(\alpha_{1}, \alpha_{2}\right)$ is the partition function given by,

$$
\mathcal{Z}\left(\alpha_{1}, \alpha_{2}\right)=\sum_{\mathcal{C}} \exp \left[-i \alpha_{1} E(\mathcal{C})-i \alpha_{2} A(\mathcal{C})\right]
$$

Since both energy and the observable are extensive quantities the sum in (6) is dominated, in the limit of large system size, by the largest contribution, and $\mathcal{Z}$ is exponentially large in the system size. The function $\mathcal{S}\left(E, A, \alpha_{1}, \alpha_{2}\right)$ is then an extensive quantity and, in that limit, the integrations can be done selecting the dominant contribution using the saddle point method:

$$
\frac{\partial \mathcal{S}\left(E, A, \alpha_{1}, \alpha_{2}\right)}{\partial \alpha_{1}}=\frac{\partial \mathcal{S}\left(E, A, \alpha_{1}, \alpha_{2}\right)}{\partial \alpha_{2}}=0
$$

which leads to the saddle point equations

$$
\begin{aligned}
& E=\frac{1}{\mathcal{Z}\left(\alpha_{1}, \alpha_{2}\right)} \sum_{\mathcal{C}} E(\mathcal{C}) \exp \left[-i \alpha_{1} E(\mathcal{C})-i \alpha_{2} A(\mathcal{C})\right] \equiv\langle E\rangle \\
& A=\frac{1}{\mathcal{Z}\left(\alpha_{1}, \alpha_{2}\right)} \sum_{\mathcal{C}} A(\mathcal{C}) \exp \left[-i \alpha_{1} E(\mathcal{C})-i \alpha_{2} A(\mathcal{C})\right] \equiv\langle A\rangle
\end{aligned}
$$


Reality of $\Omega(E, A)$ implies that the solution $\alpha_{1}^{*}(E, A), \alpha_{2}^{*}(E, A)$ of the saddle point equations must be pure imaginary: $\alpha_{1}^{*}=-i \beta, \alpha_{2}^{*}=-i \mu$ with $\beta$ and $\mu$ real numbers. The entropy (3) is given by the value of $\mathcal{S}\left(E, A, \alpha_{1}, \alpha_{2}\right)$ evaluated at the saddle point:

$$
S(E, A)=\mathcal{S}(E, A,-i \beta,-i \mu)=\beta E+\mu A+\ln \mathcal{Z}(\beta, \mu) .
$$

We can now ask the following question. What is best choice of the value of $A(\mathcal{C})$ for which $S$ attains its maximum? Stationarity of $\mathcal{S}$ with respect to $\alpha_{1}$ and $\alpha_{2}$ at the saddle point implies

$$
\begin{aligned}
& \frac{\partial S(E, A)}{\partial E}=\beta \\
& \frac{\partial S(E, A)}{\partial A}=\mu
\end{aligned}
$$

so that the maximum entropy assumption requires $\mu=0$, i.e., the entropy $S(E, A)$ must be stationary with respect to variations of $A$. For any energy $E$ the best choice of $A$ is then:

$$
A=\langle A\rangle=\frac{1}{\mathcal{Z}(\beta)} \sum_{\mathcal{C}} A(\mathcal{C}) \exp [-\beta E(\mathcal{C})]
$$

where

$$
\mathcal{Z}(\beta)=\sum_{\mathcal{C}} \exp [-\beta E(\mathcal{C})]=\exp [-\beta F(\beta)]
$$

The value of the entropy is

$$
S(E)=\beta E+\ln \mathcal{Z}(\beta)
$$

and is independent on $A$ as required from stationarity. Finally from (11) it follows that $\beta^{-1}$ can be identified with the temperature $T$ of equilibrium thermodynamics, while insertion of (14) into (15) yields the thermodynamic relation $F(\beta)=E(T)-T S(E)$ identifying $F(\beta)$ with the Helmholtz free energy. Equations (11), (13), (14) and (15) define the canonical ensemble.

At difference with the microcanonical ensemble the measure of the canonical ensemble is not restricted on states of constant energy. All possible states $\mathcal{C}$ enter but with a weight proportional to $\exp [-\beta E(\mathcal{C})]$. For a given temperature, however, only states with energy $E=\langle E\rangle$ given by [13) for $A(\mathcal{C})=E(\mathcal{C})$ [See [8] ] significantly contribute to the measure. The temperature can be seen as a Lagrange multiplier used to fix the value of the energy. Vice versa each value of $E$ in equilibrium selects a temperature $T$ through (11).

\subsection{Einstein fluctuation theory}

A key contribution in the development of equilibrium statistical mechanics is the statistical theory of fluctuations developed by Einstein [21]. In the previous section we have seen that in equilibrium the value of any observable $A(\mathcal{C})$ is given by (13), which for the purpose of this section will be denoted by $A_{\text {eq }}$. The equilibrium value corresponds to the most probable value of $A(\mathcal{C})$, i.e., to the value of $A(\mathcal{C})$ which has the overwhelming probability to be seen in equilibrium. The same considerations apply to the energy $E(\mathcal{C})$ in the canonical ensemble.

We may then ask what is the probability of observing a value of $A(\mathcal{C})$ different from the equilibrium value. This probability is simply proportional to the number of configurations with $A(\mathcal{C})=A$ which from (31) is

$$
P(\delta A) \propto \Omega\left(E_{\mathrm{eq}}, A=A_{\mathrm{eq}}+\delta A\right) \propto \exp \left[S\left(E_{\mathrm{eq}}, A_{\mathrm{eq}}+\delta A\right)\right]
$$


where following our notation $E_{\text {eq }}$ is equilibrium energy. For small value of the fluctuations $\delta A$ the exponent can be expanded and using stationarity of the entropy with respect to variation of $A$ we get,

$$
S\left(E_{\mathrm{eq}}, A\right)=S\left(E_{\mathrm{eq}}, A_{\mathrm{eq}}\right)+\frac{(\delta A)^{2}}{2}\left(\frac{\partial^{2} S}{\partial A^{2}}\right)_{A=A_{\mathrm{eq}}}+O(\delta A)^{3}
$$

If $A_{\mathrm{eq}}$ is a maximum, then a necessary condition is

$$
\left(\frac{\partial^{2} S}{\partial A^{2}}\right)_{A=A_{\mathrm{eq}}}=-\frac{1}{T \chi_{A}}<0
$$

From (16) we finally obtain,

$$
P(\delta A)=\frac{1}{\sqrt{2 \pi T \chi_{A}}} \exp \left[-\frac{(\delta A)^{2}}{2 T \chi_{A}}\right]
$$

As $\chi_{A}$ is an extensive quantity only subextensive fluctuations $\delta A \sim \sqrt{V}$, where $V$ is the system size, have finite probability in equilibrium. This justifies the most probable character of the equilibrium value $A_{\text {eq }}$.

The quantity $\chi_{A}$ is called susceptibility, and from (19) is related to fluctuations of $A$ through

$$
T \chi_{A}=\left\langle A^{2}\right\rangle-\langle A\rangle^{2} .
$$

This relation is the simplest form of the static Fluctuation-Dissipation Theorem (FDT) which relates the magnitude of thermal fluctuations with the response of the system to a (small) perturbation.

Suppose we add a constant perturbation $-\epsilon A(\mathcal{C})$ to the energy $E(\mathcal{C})$. Then in the new equilibrium value $\langle A\rangle_{\epsilon}$ of $A$ is [See [13)]

$$
\langle A\rangle_{\epsilon}=\frac{\sum_{\mathcal{C}} A(\mathcal{C}) \exp [-\beta E(\mathcal{C})+\beta \epsilon A(\mathcal{C})]}{\sum_{\mathcal{C}} \exp [-\beta E(\mathcal{C})+\beta \epsilon A(\mathcal{C})]} .
$$

The susceptibility $\chi_{A}$ is defined as the variation of $\langle A\rangle$ induced by a small perturbation

$$
\chi_{A}=\left.\frac{\partial\langle A\rangle_{\epsilon}}{\partial \epsilon}\right|_{\epsilon=0}
$$

and hence measures the response of the system to the perturbation. Inserting (21) into (22) a straightforward calculation leads to (20).

The FDT formula (20) is non-trivial result, since it relates different physical processes: the susceptibility describes an extensive $O(V)$ variation of the observable $A$ while the r.h.s. of (20) describes subextensive $O(\sqrt{V})$ thermal fluctuations. This fact is at the basis of Onsager regression principle discussed in the next section.

\subsection{The Onsager regression principle: a simple derivation of the fluctuation-dissipation theorem (FDT)}

Onsager proposed 23 24 a simple derivation of FDT for time-dependent perturbations. The derivation bypasses the more cumbersome analytical developments using linear response theory formalism, the Fokker-Planck equation or the generalized master-equation approach.

Onsager derivation is based on the following regression principle: If a system initially in an equilibrium state 1 is driven by an external perturbation to a different 


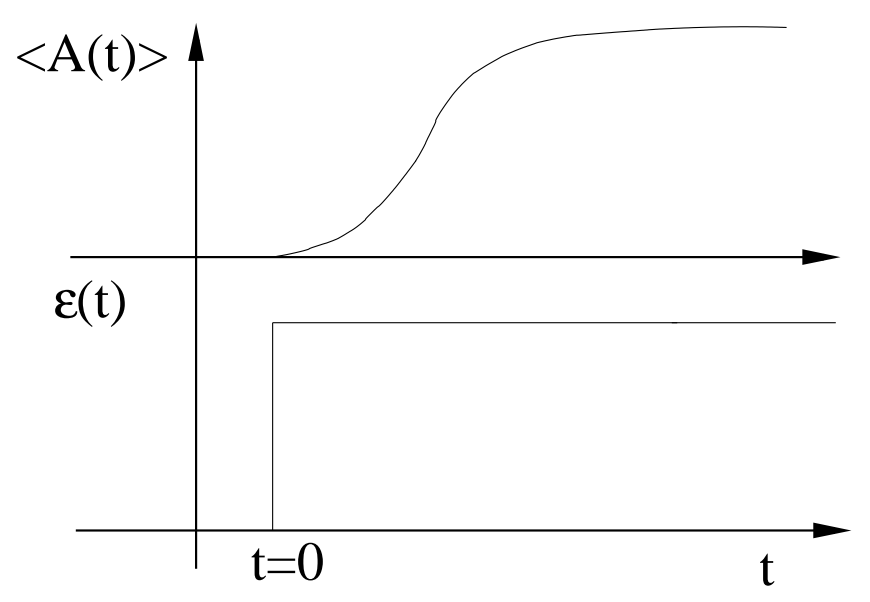

Figure 1. Perturbation $\epsilon(t)$ and typical evolution curve for $\langle A(t)\rangle_{\epsilon}$.

equilibrium state 2 , then the evolution of the system from state 1 toward state 2 in the presence of the perturbation can be treated as a spontaneous equilibrium fluctuation (in the presence of the perturbation) from the (now) non-equilibrium state 1 to the (now) equilibrium state 2 .

Suppose that the system is initially in equilibrium with a thermal bath at temperature $T$, then the probability distribution of system configuration $\mathcal{C}$ in state 1 is given by the canonical ensemble (13):

$$
P_{0}(\mathcal{C})=\frac{\exp [-\beta E(\mathcal{C})]}{\sum_{\mathcal{C}} \exp [-\beta E(\mathcal{C})]}
$$

The subscript ' 0 ' indicates that the system in unperturbed.

At time $t=0$ a constant perturbation coupled to the observable $B(\mathcal{C})$ is applied to the system changing its energy into

$$
E_{\epsilon}(\mathcal{C})=E(\mathcal{C})-\epsilon(t) B(\mathcal{C}),
$$

where $\epsilon(t)=\epsilon$ if $t>0$, and zero otherwise. The effect of the perturbation can be monitored by looking at the evolution of the expectation value $\langle A(t)\rangle_{\epsilon}$ of an observable $A(\mathcal{C})$, not necessarily equal to $B(\mathcal{C})$, from the equilibrium value in state 1 $\langle A(t=0)\rangle_{\epsilon}=\langle A\rangle_{0}$ toward the new equilibrium value in state 2. The shape of $\epsilon(t)$ and a typical evolution of $\langle A(t)\rangle_{\epsilon}$ are shown in Figure 1

The expectation value of $\langle A(t)\rangle_{\epsilon}$ is given by the average over all possible dynamical paths originating from initial configurations weighted with the probability distribution (23):

$$
\langle A(t)\rangle_{\epsilon}=\sum_{\mathcal{C}, \mathcal{C}_{0}} A(\mathcal{C}) P_{\epsilon}\left(\mathcal{C}, t \mid \mathcal{C}_{0}, 0\right) P_{0}\left(\mathcal{C}_{0}\right)
$$

where $P_{\epsilon}\left(\mathcal{C}, t \mid \mathcal{C}_{0}, 0\right)$ is the conditional probability for the evolution from the configuration $\mathcal{C}_{0}$ at time $t=0$ to the configuration $\mathcal{C}$ at time $t$. If $\epsilon=0$ the expectation value becomes time independent since the initial state is in equilibrium and $P_{0}\left(\mathcal{C}, t \mid \mathcal{C}_{0}, 0\right)$ describes spontaneous equilibrium fluctuations.

The Onsager's regression principle asserts that the conditional probabilities after having applied the perturbation are equal to those of spontaneous equilibrium 
fluctuations in state 2. Hence since the state 2 is still described by the canonical ensemble (23), but with the energy (24) now including the perturbation term, then

$$
P_{\epsilon}\left(\mathcal{C}, t \mid \mathcal{C}_{0}, 0\right)=P_{0}\left(\mathcal{C}, t \mid \mathcal{C}_{0}, 0\right) \exp \left\{\beta \epsilon\left[B(\mathcal{C})-B\left(\mathcal{C}_{0}\right]\right\} .\right.
$$

where the r.h.s of this equations is just the product of the spontaneous equilibrium fluctuations conditional probabilities $P_{0}\left(\mathcal{C}, t \mid \mathcal{C}_{0}, 0\right)$ in state 1 corrected by the presence of the perturbation term $\epsilon B(\mathcal{C}) \ddagger$. Inserting (26) into (25) and expanding the exponential up to the linear order we get,

$$
\begin{aligned}
\langle A(t)\rangle_{\epsilon}-\langle A\rangle_{0} & =\beta \epsilon \sum_{\mathcal{C}, \mathcal{C}_{0}} A(\mathcal{C})\left[B(\mathcal{C})-B\left(\mathcal{C}_{0}\right)\right] P_{0}\left(\mathcal{C}_{0}\right) \\
& =\beta \epsilon\left[\langle A(t) B(t)\rangle_{0}-\langle A(t) B(0)\rangle_{0}\right] .
\end{aligned}
$$

If we define the correlation function and time-dependent susceptibility as

$$
\begin{aligned}
& C_{A, B}(t, s)=\langle A(t) B(s)\rangle_{0} \\
& \chi_{A, B}(t)=\lim _{\epsilon \rightarrow 0} \frac{\langle A(t)\rangle_{\epsilon}-\langle A\rangle_{0}}{\epsilon}
\end{aligned}
$$

then from (27) we get the integrated form of the FDT relation

$$
\chi_{A, B}(t)=\beta\left[C_{A, B}(t, t)-C_{A, B}(t, 0)\right]
$$

The static form of the FDT (20) is easily obtained from (30) by taking $A=B$ and the limit $t \rightarrow \infty$. In this case $\chi_{A, A}(t) \rightarrow \chi_{A}$ [cfr. (22)] and $C_{A A}(t, t)=\left\langle A^{2}\right\rangle_{0}$ while $C_{A A}(t, 0) \rightarrow\langle A\rangle_{0}^{2}$ as correlations factorize for infinitely separated times.

Sometimes the FDT relation is written in a differential form by considering the two-times response or retarded Green function:

$$
R_{A, B}(t, s)=\frac{\delta\langle A(t)\rangle}{\delta \epsilon(s)}, \quad t>s
$$

which gives the response to an impulsive perturbation $\epsilon(s)$ acting at time $s$. Causality imposes that the response function $R_{A, B}(t, s)$ is zero for $t<s$ : perturbations cannot propagate backward in time. The susceptibility $\chi_{A, B}(t)$ is the integral of the response function $R_{A, B}(t, s)$ then, using (30):

$$
\begin{aligned}
\int_{0}^{t} R_{A, B}(t, s) d s & =\chi_{A, B}(t) \\
& =\beta\left[C_{A, B}(0)-C_{A, B}(t)\right] \\
& =\beta \int_{0}^{t} \frac{\partial}{\partial s} C_{A, B}(t, s) d s .
\end{aligned}
$$

The last equality, and the arbitrarity of time $t$, implies that

$$
R_{A, B}(t, s)=\beta \frac{\partial}{\partial s} C_{A, B}(t, s) \theta(t-s)
$$

This is the differential form of the FDT relation.

$\ddagger$ The relation 26 is only valid for $\mathcal{C}_{0} \neq \mathcal{C}$. Indeed, the normalization condition of conditional probabilities $\sum_{\mathcal{C}} P_{\epsilon}\left(\mathcal{C}, t \mid \mathcal{C}_{0}, 0\right)=1$ implies that $P_{\epsilon}(\mathcal{C}, t \mid \mathcal{C}, 0) \neq P_{0}(\mathcal{C}, t \mid \mathcal{C}, 0)$ so relation [26] does not hold for $\mathcal{C}_{0}=\mathcal{C}$. The difference between both probabilities $P_{\epsilon}(\mathcal{C}, t \mid \mathcal{C}, 0), P_{0}(\mathcal{C}, t \mid \mathcal{C}, 0)$ does not matter as the transition $\mathcal{C} \rightarrow \mathcal{C}$ does not contribute to the response function in 27] 


\section{The Master Equation Approach}

In this section we introduce the Master equation for the dynamical evolution of a generic system and show how the FDT arises within this approach. Besides the previous derivation in Section 2.3 many other derivations of the FDT exist. We give a few collection of references where these are presented. Derivations can be classified in two families: deterministic or stochastic. Deterministic approaches are linear response theory [25], operator formalism for master equations [26] and quantum statistical mechanics [27. Stochastic approaches are the Langevin and Fokker-Planck equations 28. Here we present a stochastic derivation which is convenient for the purpose of the present review.

\subsection{The Master Equation (ME)}

Any dynamical law describing the evolution of a system is a rule which for each system configuration $\mathcal{C}$ associates a new configuration $\mathcal{C}^{\prime}$. The time is just a label for bookkeeping the sequence so generated. Therefore to simplify the presentation and the notation we shall consider the time as an integer variable giving the equivalent

expressions for the continuous limit when needed. This picture has also the advantage of being more closely related to numerical simulations since all numerical methods use discrete time schemes.

The dynamics can be encoded into the conditional or transition probability $W\left(\mathcal{C}, t \mid \mathcal{C}^{\prime}, t-1\right)$ of going from configuration $\mathcal{C}^{\prime}$ at time $t-1$ to configuration $\mathcal{C}$ at time $t$. Indeed if $P(\mathcal{C}, t)$ denotes the probability that the system at time $t$ is in the configuration $\mathcal{C}$, then from the Bayes theorem it follows that

$$
P(\mathcal{C}, t)=\sum_{\mathcal{C}^{\prime}} W\left(\mathcal{C}, t \mid \mathcal{C}^{\prime}, t-1\right) P\left(\mathcal{C}^{\prime}, t-1\right) .
$$

and the $W\left(\mathcal{C}, t \mid \mathcal{C}^{\prime}, t-1\right)$ together with the initial condition $P(\mathcal{C}, 0)$ fully define the dynamical evolution of the system in the phase space. Equation (34) is an identity valid for all processes and is the first of a hierarchy of equations for joint probabilities. Only if the process is Markovian, i.e., only if the conditional probability is determined entirely by the knowledge of the most recent past, then the hierarchy can be closed. Equation (34) and probability conservation at all times $\left(\sum_{\mathcal{C}^{\prime}} P\left(\mathcal{C}^{\prime}, t\right)=1\right)$ implies that $W\left(\mathcal{C}, t \mid \mathcal{C}^{\prime}, t-1\right)$ must satisfy the normalization condition

$$
\sum_{\mathcal{C}^{\prime}} W\left(\mathcal{C}^{\prime}, t \mid \mathcal{C}, t-1\right)=1 \quad \text { for all } \mathcal{C} \text { and } t .
$$

In the continuous time limit (34) is not well defined. To have an expression valid in this limit one then considers the variation of $P(\mathcal{C}, t)$ between two successive times which, using the normalization condition (35), reads

$$
\begin{aligned}
& P(\mathcal{C}, t+1)-P(\mathcal{C}, t)= \\
& \quad \sum_{\mathcal{C}^{\prime}} W\left(\mathcal{C}, t+1 \mid \mathcal{C}^{\prime}, t\right) P\left(\mathcal{C}^{\prime}, t\right)-\sum_{\mathcal{C}^{\prime}} W\left(\mathcal{C}^{\prime}, t+1 \mid \mathcal{C}, t\right) P(\mathcal{C}, t) .
\end{aligned}
$$

Dividing both sides of this equality for the time increment $\Delta t$ and and taking it to zero we get the Master Equation (ME)

$$
\frac{\partial P(\mathcal{C}, t)}{\partial t}=\sum_{\mathcal{C}^{\prime}} W\left(\mathcal{C} \mid \mathcal{C}^{\prime} ; t\right) P\left(\mathcal{C}^{\prime}, t\right)-\sum_{\mathcal{C}^{\prime}} W\left(\mathcal{C}^{\prime} \mid \mathcal{C} ; t\right) P(\mathcal{C}, t)
$$


where $W\left(\mathcal{C}^{\prime} \mid \mathcal{C} ; t\right)=\lim _{\Delta t \rightarrow 0} W\left(\mathcal{C}, t+\Delta t \mid \mathcal{C}^{\prime}, t\right) / \Delta t$ is called transition rate and gives the transition probability per unit of time. Solving the ME is often an extremely difficult and unfordable task, even in the Markovian case.

Loosely speaking the transition probability $W\left(\mathcal{C}, t \mid \mathcal{C}^{\prime}, t-1\right)$ can be seen as a transition rate for a unit time interval $(\Delta t=1)$, thus in what follows we shall not make distinction between transition probabilities and transition rates and shall call them generically transition rates using for both the notation $W\left(\mathcal{C}^{\prime} \mid \mathcal{C} ; t\right)$. Which one is the appropriate will be clear from the context.

Transition rates depend on the specific dynamical rules and hence by the Hamiltonian and eventual constraints (holonomic or non-holonomic). Let the system under consideration be described by an Hamiltonian which can depend on time through a set of time-dependent external parameters $\lambda_{t}^{j}$. For instance, $\lambda_{t}$ may denote an time-dependent external pressure applied to a liquid or a time varying electric or magnetic field applied in a dielectric or a magnetic medium. We shall denote the set of these parameters by the vector $\boldsymbol{\lambda}_{t}$ and the Hamiltonian by $\mathcal{H}_{\boldsymbol{\lambda}_{t}}(\mathcal{C})$ to indicate the time dependence through $\boldsymbol{\lambda}_{t}$. Accordingly we shall also denote $W\left(\mathcal{C} \mid \mathcal{C}^{\prime}, t\right)$ by $W_{\boldsymbol{\lambda}_{t}}\left(\mathcal{C} \mid \mathcal{C}^{\prime}\right)$. If the Hamiltonian is time-independent either $\boldsymbol{\lambda}_{t}$ or just the subindex $t$ will be dropped, depending on the context.

Regardless of their form the transition rates must satisfy the following requirements:

- Non-negativeness and normalization. The $W_{\boldsymbol{\lambda}_{t}}\left(\mathcal{C} \mid \mathcal{C}^{\prime} ; t\right)$ are probabilities so they must be non-negative and satisfy the normalization condition (35).

- Ergodicity. Transition rates must be such that starting from any configuration any other configuration of a finite system can be visited in a finite time. For continuous variables the condition is stated by considering an arbitrary finite phase space region around a given point ('neighborhood').

- Detailed balance. If the $\boldsymbol{\lambda}$ are time-independent the equilibrium distribution $P_{\boldsymbol{\lambda}}^{\mathrm{eq}}(\mathcal{C})$ is the stationary solution of the ME (37). A sufficient condition for this is that the transition rates be time-independent and

$$
\frac{W_{\boldsymbol{\lambda}}\left(\mathcal{C}^{\prime} \mid \mathcal{C}\right)}{W_{\boldsymbol{\lambda}}\left(\mathcal{C} \mid \mathcal{C}^{\prime}\right)}=\frac{P_{\boldsymbol{\lambda}}^{\mathrm{eq}}\left(\mathcal{C}^{\prime}\right)}{P_{\boldsymbol{\lambda}}^{\mathrm{eq}}(\mathcal{C})}
$$

This condition receives the name of detailed balance. Different equilibrium ensembles are thus encoded into the different forms of transition rates. The Perron-Frobenius theorem assures [29] that this condition, together with nonnegativeness, normalization and ergodicity guarantees that the equilibrium distribution in a finite system is reached in a finite time.

- Causality. This is an important assumption for the time-dependent transition rates and means that future is only determined by the past, i.e., a perturbation applied at a given time can only propagate forward in time and not backward. Consequence of this is that the transition rates must depend only on the values of $\boldsymbol{\lambda}_{t}$ taken at the lowest time $t$.

For arbitrary time-dependent $\boldsymbol{\lambda}_{t}$ the stationary solution of ME will in general also depend on time. In this case, however, the previous conditions, and in particular the detailed balance condition, are not enough for determining the stationary state which in general will not be Gibbsian, i.e., not described by the Boltzmann-Gibbs distribution. Only when $\boldsymbol{\lambda}_{t}$ can be treated as small perturbations some predictions can be obtained from the linear response theory. 


\subsection{Correlations, responses and the FDT}

Consider two arbitrary observables $A(\mathcal{C}), B(\mathcal{C})$ which for simplicity are assumed timeindependent. These can be either local or global quantities defined over a microscopic or macroscopic region respectively. For instance, in a liquid an observable could be the local density at a point or the total mass of a given macroscopic region. In systems with discrete variables such as magnetic systems, it can be a spin of a given magnetic atom or the magnetization of a macroscopic part of the system.

The two-times correlation function $C_{A, B}(t, s)$ between $A(t)$ and $B(s)$ is defined as the average $\langle A(t) B(s)\rangle$ over all possible dynamical paths from time 0 to time $t$ and all possible initial conditions weighted by the probability distribution $P(\mathcal{C}, 0)$,

$$
C_{A, B}(t, s)=\langle A(t) B(s)\rangle=\sum_{\mathcal{C}, \mathcal{C}^{\prime}} A\left(\mathcal{C}^{\prime}\right) P\left(\mathcal{C}^{\prime}, t \mid \mathcal{C}, s\right) B(\mathcal{C}) P(\mathcal{C}, s)
$$

where $P\left(\mathcal{C}^{\prime}, t \mid \mathcal{C}, s\right)$ is the conditional probability to evolve from $\mathcal{C}$ at time $s$ to $\mathcal{C}^{\prime}$ at later time $t$. Unless otherwise stated, in what follows we shall adopt the convention that $t \geq s$.

To simplify the notation we switch to discrete (integer) time variable so that a dynamical path from time 0 to time $t$ is given by a sequence of $t$ configurations $\left\{\mathcal{C}_{0}, \mathcal{C}_{1}, \ldots, \mathcal{C}_{t}\right\}$. along which $\boldsymbol{\lambda}$ takes the sequence of values $\left\{\boldsymbol{\lambda}_{0}, \boldsymbol{\lambda}_{1}, \ldots, \boldsymbol{\lambda}_{t}\right\}$. In this case, using (34), the correlation can be easily rewritten in term of transition rates as

$$
C_{A, B}(t, s)=\sum_{\mathcal{C}_{s}, \ldots, \mathcal{C}_{t}} A\left(\mathcal{C}_{t}\right)\left[\prod_{k=s}^{t-1} W_{\boldsymbol{\lambda}_{k}}\left(\mathcal{C}_{k+1} \mid \mathcal{C}_{k}\right)\right] B\left(\mathcal{C}_{s}\right) P\left(\mathcal{C}_{s}, s\right)
$$

where we have used the short-hand notation $W_{\boldsymbol{\lambda}_{k}}\left(\mathcal{C}_{k+1} \mid \mathcal{C}_{k}\right) \equiv W_{\boldsymbol{\lambda}_{k}}\left(\mathcal{C}_{k+1} \mid \mathcal{C}_{k} ; k\right)$.

In equilibrium correlations satisfy the time-translational invariance (TTI) property: $C_{A, B}(t, s)=C_{A, B}(t-s)$. Indeed in this case $P\left(\mathcal{C}_{s}, s\right)$ is replaced by $P^{\mathrm{eq}}\left(\mathcal{C}_{s}\right)$ and the transition rates satisfy the detailed balance condition (38):

$$
W_{\boldsymbol{\lambda}}\left(\mathcal{C}_{k+1} \mid \mathcal{C}_{k}\right)=W_{\boldsymbol{\lambda}}\left(\mathcal{C}_{k} \mid \mathcal{C}_{k+1}\right) \frac{P_{\boldsymbol{\lambda}}^{\mathrm{eq}}\left(\mathcal{C}_{k+1}\right)}{P_{\boldsymbol{\lambda}}^{\mathrm{eq}}\left(\mathcal{C}_{k}\right)} .
$$

Inserting this relation into (40) the factors $P^{\mathrm{eq}}\left(\mathcal{C}_{k}\right)$ in the numerator and denominator of the product cancel one by one and, exchanging the indexes $t \leftrightarrow s$, we obtain

$$
\begin{aligned}
C_{A, B}(t, s)= & \sum_{\mathcal{C}_{s}, \ldots, \mathcal{C}_{t}} A\left(\mathcal{C}_{t}\right) P_{\boldsymbol{\lambda}}^{\mathrm{eq}}\left(\mathcal{C}_{t}\right)\left[\prod_{k=s}^{t-1} W_{\boldsymbol{\lambda}}\left(\mathcal{C}_{k+1} \mid \mathcal{C}_{k}\right)\right] B\left(\mathcal{C}_{s}\right) \\
& =(t \leftrightarrow s) \\
& =\sum_{\mathcal{C}_{t}, \ldots, \mathcal{C}_{s}} B\left(\mathcal{C}_{t}\right)\left[\prod_{k=t}^{s-1} W_{\boldsymbol{\lambda}}\left(\mathcal{C}_{k} \mid \mathcal{C}_{k+1}\right)\right] A\left(\mathcal{C}_{s}\right) P_{\boldsymbol{\lambda}}^{\mathrm{eq}}\left(\mathcal{C}_{s}\right) \\
& =\langle B(t) A(s)\rangle \\
& =C_{B, A}(t, s) .
\end{aligned}
$$

which implies that $C_{A, B}(t, s)=C_{A, B}(t-s)$.

The correlation function $C_{A, B}(t, s)$ is a measure of how the system loses memory of its previous past history and hence decays for large time separations. To measure how a system responds to external perturbations one introduces the response functions. Similar to correlations, responses also tend to decay with time because the effect of the perturbation is progressively forgotten in a thermal environment. However there 
is an important difference between correlations and responses: causality. While two observables can be correlated forward or backward in time, a perturbation cannot propagate backward in time and the response of the system for times before the perturbation is applied must be zero. Nevertheless despite this difference response and correlations can be treated on equal footing by employing a supersymmetric formalism. The interested reader can find more details, e.g. in the classical book by Zinn-Justin 28].

To study the response of the system to an external perturbation we assume that at time $s$ an impulsive perturbation of small intensity $\epsilon$ is applied to the observable $B(\mathcal{C})$ and measure the variation of the average value of an observable $A(\mathcal{C})$ at later times. The response function $R_{A, B}(t, s)$ is defined in the limit of vanishing perturbation strength as

$$
R_{A, B}(t, s)=\lim _{\epsilon \rightarrow 0} \frac{\langle A(t)\rangle_{\epsilon_{s}}-\langle A(t)\rangle_{0}}{\epsilon}, \quad t>s
$$

with

$$
\begin{aligned}
\langle A(t)\rangle_{\epsilon_{s}} & =\sum_{\mathcal{C}} A(\mathcal{C}) P_{\epsilon_{s}}(\mathcal{C}, t), \\
\langle A(t)\rangle_{0} & =\sum_{\mathcal{C}} A(\mathcal{C}) P_{0}(\mathcal{C}, t) .
\end{aligned}
$$

where $P_{\epsilon_{s}}(\mathcal{C}, t)$ and $P_{0}(\mathcal{C}, t)$ are the probability that the system is in the configuration $\mathcal{C}$ at time $t>s$ in the perturbed and unperturbed case, respectively. If the system is described by the unperturbed Hamiltonian $\mathcal{H}_{0}(\mathcal{C})$, then in presence of the perturbation the Hamiltonian becomes:

$$
\mathcal{H}_{\epsilon_{s}}(\mathcal{C})=\mathcal{H}_{0}(\mathcal{C})-\delta_{t, s} \epsilon B(\mathcal{C})
$$

By using (34) and the fact that the Hamiltonians only differ at time $s$ when the impulse is applied, the probabilities $P_{\epsilon_{s}}(\mathcal{C}, t)$ and $P_{0}(\mathcal{C}, t)$ can be written as:

$$
\begin{aligned}
& P_{\epsilon_{s}}\left(\mathcal{C}_{t}, t\right)=\sum_{\mathcal{C}_{s}, \cdots, \mathcal{C}_{t-1}}\left[\prod_{k=s+1}^{t-1} W_{0}\left(\mathcal{C}_{k+1} \mid \mathcal{C}_{k}\right)\right] W_{\epsilon}\left(\mathcal{C}_{s+1} \mid C_{s}\right) P_{0}\left(\mathcal{C}_{s}, s\right) \\
& P_{0}\left(\mathcal{C}_{t}, t\right)=\sum_{\mathcal{C}_{s}, \cdots, \mathcal{C}_{t-1}}\left[\prod_{k=s+1}^{t-1} W_{0}\left(\mathcal{C}_{k+1} \mid \mathcal{C}_{k}\right)\right] W_{0}\left(\mathcal{C}_{s+1} \mid C_{s}\right) P_{0}\left(\mathcal{C}_{s}, s\right)
\end{aligned}
$$

where $W_{0, \epsilon}$ denotes the transition rates in the unperturbed/perturbed case, and we have used the short-hand notation $W\left(\mathcal{C}_{k+1} \mid \mathcal{C}_{k}\right) \equiv W\left(\mathcal{C}_{k+1} \mid \mathcal{C}_{k} ; k\right)$.

Because we are interested in the $\epsilon \rightarrow 0$ limit the transition rates in the perturbed and unperturbed case can be related by expanding the detailed balance condition (38) for the perturbed state around $\epsilon=0$ up to the first order in $\epsilon$,

$$
\begin{aligned}
\frac{W_{\epsilon}\left(\mathcal{C}^{\prime} \mid \mathcal{C}\right)}{W_{\epsilon}\left(\mathcal{C} \mid \mathcal{C}^{\prime}\right)}= & \frac{P_{\epsilon}^{\mathrm{eq}}\left(\mathcal{C}^{\prime}\right)}{P_{\epsilon}^{\mathrm{eq}}(\mathcal{C})} \\
& =\frac{W_{0}\left(\mathcal{C}^{\prime} \mid \mathcal{C}\right)}{W_{0}\left(\mathcal{C} \mid \mathcal{C}^{\prime}\right)}\left\{1+\left.\epsilon \frac{\partial}{\partial \epsilon} \ln \left[P_{\epsilon}^{\mathrm{eq}}\left(\mathcal{C}^{\prime}\right) / P_{\epsilon}^{\mathrm{eq}}(\mathcal{C})\right]\right|_{\epsilon=0}+\mathcal{O}\left(\epsilon^{2}\right)\right\}
\end{aligned}
$$

where $P_{\epsilon}^{\text {eq }}(\mathcal{C})$ is the equilibrium probability distribution in the perturbed state $\dagger$. In general we can write,

$$
\log \left[P_{\epsilon}^{\mathrm{eq}}(\mathcal{C})\right]=\log \left[P_{0}^{\mathrm{eq}}(\mathcal{C})\right]+\phi_{0}-\phi_{\epsilon}+\beta \epsilon B(\mathcal{C})
$$

$\dagger$ An observation concerning (49) and its relation with the Onsager postulate 26) is important. Ideally, in order to demonstrate FDT, one would like to have a relation similar to 26 relating the unperturbed and the perturbed rates rather than a relation between the forward and backward 
where $\phi$ denotes the corresponding thermodynamic potential. For instance, in the canonical ensemble it corresponds to minus the Helhmoltz free energy $F$ while in the grandcanonical ensemble it corresponds to the grandcanonical potential given by the pressure times the volume.

Using (49) we finally obtain to the leading order in $\epsilon$,

$$
\begin{aligned}
W_{\epsilon}\left(\mathcal{C}^{\prime} \mid \mathcal{C}\right)-W_{0}\left(\mathcal{C}^{\prime} \mid \mathcal{C}\right) & =\left[\frac{W_{\epsilon}\left(\mathcal{C} \mid \mathcal{C}^{\prime}\right)}{W_{0}\left(\mathcal{C} \mid \mathcal{C}^{\prime}\right)}-1\right] W_{0}\left(\mathcal{C}^{\prime} \mid \mathcal{C}\right) \\
& +\left.\epsilon \frac{\partial}{\partial \epsilon} \ln \left[P_{\epsilon}^{\mathrm{eq}}\left(\mathcal{C}^{\prime}\right) / P_{\epsilon}^{\mathrm{eq}}(\mathcal{C})\right]\right|_{\epsilon=0} W_{0}\left(\mathcal{C}^{\prime} \mid \mathcal{C}\right)
\end{aligned}
$$

The physical meaning of the two terms appearing in this expression is different. The first term, absent in the Onsager's postulate (26), accounts for the variation due to the change in the transition rates and does not directly depend upon the particular form of the equilibrium distribution. The second term depends directly on the equilibrium distribution. This distinction is important since they give different contributions to the response. For the response function (43) to be well defined the difference $\langle A(t)\rangle_{\epsilon}-\langle A(t)\rangle_{0}$ must be at least linear in $\epsilon$. This requirement is at the roots of the applicability of linear response theory and implies that both terms in (51) must be at least linear in $\epsilon$. Concerning the first term, it is required that the difference $W_{\epsilon}\left(\mathcal{C} \mid \mathcal{C}^{\prime}\right) / W_{0}\left(\mathcal{C} \mid \mathcal{C}^{\prime}\right)-1$ to be linear in $\epsilon$, so the transition rates must change linearly with $\epsilon$ when the system is perturbed. This means that, during the dynamics, and after applying the perturbation, one cannot switch arbitrarily from one class of dynamics to another class of dynamics in a random fashion. To better illustrate what this means let us consider a Monte Carlo stochastic dynamics. The are different possible algorithms or transition rules which fulfill detailed balance (e.g. Metropolis, heat-bath, Glauber). One could imagine of switching randomly from one algorithm to another one while doing the dynamics. Nothing forbids this quite artificial choice. But, when measuring the response function, it is required that the same time sequence of algorithms must be used for the unperturbed and perturbed dynamical evolutions. Usually, the same algorithm is chosen in a given simulation so one does not care about this subtlety. For the second term, we require that $\left.(\partial / \partial \epsilon) \ln \left[P_{\epsilon}^{\mathrm{eq}}\left(\mathcal{C}^{\prime}\right) / P_{\epsilon}^{\mathrm{eq}}(\mathcal{C})\right]\right|_{\epsilon=0}$ be finite. Inspection of (50) reveals that this holds if the observable $B(C)$ does not jump discontinuously when the perturbation is switched on. This condition requires that the system is not at a first order transition point. This situation is encountered, for instance, by perturbing the Ising model at zero temperature with a uniform magnetic field.

Inserting (51) into (43) the response function decomposes into two parts,

$$
R_{A, B}(t, s)=R_{A, B}^{(1)}(t, s)+R_{A, B}^{(2)}(t, s)
$$

where,

$R_{A, B}^{(1)}(t, s)=\lim _{\epsilon \rightarrow 0} \frac{1}{\epsilon} \sum_{\mathcal{C}_{s}, \ldots, \mathcal{C}_{t}} A\left(\mathcal{C}_{t}\right)\left[\prod_{k=s+1}^{t-1} W_{0}\left(\mathcal{C}_{k+1} \mid \mathcal{C}_{k}\right)\right]$

rates as given in (49). However, such a relation does not exist as it depends upon the type of dynamics through the particular form of the transition rules. For instance, by considering rates of the type $W_{\boldsymbol{\lambda}}\left(\mathcal{C}^{\prime} \mid \mathcal{C}\right) \propto P_{\boldsymbol{\lambda}}^{\text {eq }}\left(\mathcal{C}^{\prime}\right)$, i.e. depending only upon the final configuration, one finds that (26) automatically holds. However such rates cannot be used to derive the FDT [33, (55) as they lead to the trivial identity $0=0$ because any impulse does not affect dynamics at later times $R(t, s)=0$ and there are no time correlations. In other words, the Onsager postulate extended to the non-equilibrium regime generates violation terms different to those obtained in the present approach (for instance the term $R^{(1)}$ in the r.h.s of [59]). 
Violation of FDT in glassy systems

$$
\begin{gathered}
\times\left[\frac{W_{\epsilon}\left(\mathcal{C}_{s} \mid \mathcal{C}_{s+1}\right)}{W_{0}\left(\mathcal{C}_{s} \mid \mathcal{C}_{s+1}\right)}-1\right] W_{0}\left(\mathcal{C}_{s+1} \mid \mathcal{C}_{s}\right) P_{0}\left(\mathcal{C}_{s}, s\right) \\
R_{A, B}^{(2)}(t, s)=\sum_{\mathcal{C}_{s}, \ldots, \mathcal{C}_{t}} A\left(\mathcal{C}_{t}\right)\left[\prod_{k=s+1}^{t-1} W_{0}\left(\mathcal{C}_{k+1} \mid \mathcal{C}_{k}\right)\right] \\
\times\left.\frac{\partial}{\partial \epsilon} \ln \left[P_{\epsilon}^{\mathrm{eq}}\left(\mathcal{C}^{\prime}\right) / P_{\epsilon}^{\mathrm{eq}}(\mathcal{C})\right]\right|_{\epsilon=0} W_{0}\left(\mathcal{C}_{s+1} \mid \mathcal{C}_{s}\right) P_{0}\left(\mathcal{C}_{s}, s\right)
\end{gathered}
$$

Inserting (50) for the second term we get

$$
\begin{aligned}
R_{A, B}^{(2)}(t, s)=\left\langle\left. A(t) \frac{\partial \ln P_{\epsilon}^{\mathrm{eq}}\left(\mathcal{C}_{s+1}\right)}{\partial \epsilon}\right|_{\epsilon=0}\right\rangle_{0} \\
-\left\langle\left. A(t) \frac{\partial \ln P_{\epsilon}^{\mathrm{eq}}\left(\mathcal{C}_{s}\right)}{\partial \epsilon}\right|_{\epsilon=0}\right\rangle_{0}
\end{aligned}
$$

which using (50) becomes

$$
R_{A, B}^{(2)}(t, s)=\beta\langle A(t) B(s+1)\rangle_{0}-\beta\langle A(t) B(s)\rangle_{0}
$$

In the limit of continuous time $R^{(2)}$ in (56) must be replaced by

$$
R_{A, B}^{(2)}(t, s)=\beta \frac{\partial}{\partial s}\langle A(t) B(s)\rangle_{0}
$$

The first term $R^{(1)}$ cannot be expressed in a simple form. Only in equilibrium it is possible to show that it vanishes. To prove it requires the following steps: first use the identity

$$
\prod_{k=s+1}^{t-1} W_{0}\left(\mathcal{C}_{k+1} \mid \mathcal{C}_{k}\right)=\left[\prod_{k=s+1}^{t-1} W_{0}\left(\mathcal{C}_{k} \mid \mathcal{C}_{k+1}\right)\right] \frac{P_{0}^{\mathrm{eq}}\left(\mathcal{C}_{t}\right)}{P_{0}^{\mathrm{eq}}\left(\mathcal{C}_{s+1}\right)}
$$

and then the normalization (35). Collecting all terms we finally obtain for the response function

$$
R_{A, B}(t, s)=R_{A, B}^{(1)}(t, s)+\beta \frac{\partial}{\partial s}\langle A(t) B(s)\rangle_{0}
$$

In equilibrium, besides $R^{(1)}=0$, the correlation function satisfy TTI, so from (59) we get the equilibrium FDT

$$
R_{A, B}^{\mathrm{eq}}(t-s)=\beta \frac{\partial}{\partial s} C_{A, B}^{\mathrm{eq}}(t-s)=-\beta \frac{\partial}{\partial t} C_{A, B}^{\mathrm{eq}}(t-s), \quad t>s
$$

The term $R^{(1)}$ may also vanish in the non-equilibrium state if the first term in (51) vanishes faster than linearly with $\epsilon$ for $\epsilon \rightarrow 0$. In this case (59) reduces to the usual FDT relation (33).

$$
R_{A, B}(t, s)=\beta \theta(t-s) \frac{\partial}{\partial s} C_{A, B}(t, s)
$$

Therefore, the lowest time $s$ has a special role in the relation (59) between correlations and responses. This is not a surprise since the relation has been obtained by assuming causality which privileges the lowest time. In equilibrium, the role of the lowest time disappears because the system is TTI. 


\subsection{The Component Master Equation}

Let us now divide the phase space into different non-overlapping subsets $\mathcal{R}$ that can be called regions, phases, components or domains. In what follows, if not stated otherwise, we shall use the term component. Later in Section 4.2 we will see that the reduction of the phase space by a suitable partitioning can be relevant for the study of the non-equilibrium regime in glassy systems and in particular for the understanding of the FDT relations. For the moment, however, we do not attach any physical meaning to such a partitioning, and assume it to be completely arbitrary postponing the identification of a suitable partitioning scheme for glassy systems to Section 4.5

For each partition of the phase space the probability $\mathcal{P}(\mathcal{R}, t)$ that system be in the component $\mathcal{R}$ at time $t$ is given by,

$$
\begin{aligned}
& \mathcal{P}(\mathcal{R}, t)=\sum_{\mathcal{C} \in \mathcal{R}} P(\mathcal{C}, t), \\
& \sum_{\mathcal{R}} \mathcal{P}(\mathcal{R}, t)=1
\end{aligned}
$$

where the normalization condition (63) follows from normalization of $P(\mathcal{C}, t)$ and the non-overlapping assumption on $\mathcal{R}$. The probability distribution $\mathcal{P}(\mathcal{R}, t)$ obeys the master equation obtained by projecting the (microscopic) master equation (37) over the component space. The Markovian character of the dynamics is preserved under projection. Summing (37) over all configurations $\mathcal{C}$ belonging to a given component $\mathcal{R}$ we get the Component Master Equation:

$$
\frac{\partial \mathcal{P}(\mathcal{R}, t)}{\partial t}=\sum_{\mathcal{R}^{\prime}} \mathcal{W}\left(\mathcal{R} \mid \mathcal{R}^{\prime} ; t\right) \mathcal{P}\left(\mathcal{R}^{\prime}, t\right)-\sum_{\mathcal{R}^{\prime}} \mathcal{W}\left(\mathcal{R}^{\prime} \mid \mathcal{R} ; t\right) \mathcal{P}(\mathcal{R}, t)
$$

where $\mathcal{W}\left(\mathcal{R}^{\prime} \mid \mathcal{R} ; t\right)$ are the component transition rates which in terms of the original transition rates $W\left(\mathcal{C}^{\prime} \mid \mathcal{C} ; t\right)$ read,

$$
\mathcal{W}\left(\mathcal{R}^{\prime} \mid \mathcal{R} ; t\right)=\frac{\sum_{\mathcal{C}^{\prime} \in \mathcal{R}^{\prime}, \mathcal{C} \in \mathcal{R}} W\left(\mathcal{C}^{\prime} \mid \mathcal{C}, t\right) P(\mathcal{C}, t)}{\mathcal{P}(\mathcal{R}, t)} .
$$

The component transition rates satisfies the same normalization conditions as $W$. For example in the case of discrete time we have [Cfr. [35]],

$$
\sum_{\mathcal{R}^{\prime}} \mathcal{W}\left(\mathcal{R}^{\prime}, t \mid \mathcal{R}, t-1\right)=1 \quad \text { for all } \mathcal{R} \text { and } t .
$$

However there is an important difference between the (microscopic) master equation (37) and component master equation (64): the transition rates (65) are time-dependent even in for time-independent Hamiltonians since are computed with the run-time configuration probability distribution function $P(\mathcal{C}, t)$. Direct consequence of this is that, while the properties of non-negativeness, normalization, ergodicity and causality discussed in section 3.1 for $W$ do apply to $\mathcal{W}$, the detailed balance is not valid anymore in the component space. Indeed from (65) it follows that

$$
\frac{\mathcal{W}\left(\mathcal{R}^{\prime} \mid \mathcal{R} ; t\right)}{\mathcal{W}\left(\mathcal{R} \mid \mathcal{R}^{\prime} ; t\right)}=\frac{\sum_{\mathcal{C} \in \mathcal{R}, \mathcal{C}^{\prime} \in \mathcal{R}^{\prime}} W\left(\mathcal{C}^{\prime} \mid \mathcal{C}\right) P(\mathcal{C}, t)}{\sum_{\mathcal{C} \in \mathcal{R}, \mathcal{C}^{\prime} \in \mathcal{R}^{\prime}} W\left(\mathcal{C} \mid \mathcal{C}^{\prime}\right) P\left(\mathcal{C}^{\prime}, t\right)} \times \frac{\mathcal{P}\left(\mathcal{R}^{\prime}, t\right)}{\mathcal{P}(\mathcal{R}, t)}
$$

The detailed balance condition is recovered, however, at equilibrium where $\mathcal{W}$, accordintg to 65), becomes time-independent:

$$
\mathcal{W}^{\mathrm{eq}}\left(\mathcal{R}^{\prime} \mid \mathcal{R}\right)=\frac{\sum_{\mathcal{C} \in \mathcal{R}, \mathcal{C}^{\prime} \in \mathcal{R}^{\prime}} W\left(\mathcal{C}^{\prime} \mid \mathcal{C}\right) P^{\mathrm{eq}}(\mathcal{C})}{\mathcal{P}^{\mathrm{eq}}(\mathcal{R})}
$$


where $\mathcal{P}^{\mathrm{eq}}(\mathcal{R})$ denotes the equilibrium probability distribution function in the component space associated through (62) to the phase space equilibrium probability distribution function $\mathcal{P}^{\mathrm{eq}}(\mathcal{C})$. Using the detailed balance condition (38) we obtain,

$$
\frac{\mathcal{W}^{\mathrm{eq}}\left(\mathcal{R}^{\prime} \mid \mathcal{R}\right)}{\mathcal{W}^{\mathrm{eq}}\left(\mathcal{R} \mid \mathcal{R}^{\prime}\right)}=\frac{\mathcal{P}^{\mathrm{eq}}\left(\mathcal{R}^{\prime}\right)}{\mathcal{P}^{\mathrm{eq}}(\mathcal{R})}
$$

which is the detailed balance condition in the component space.

In conclusion, in the component space the rates $\mathcal{W}$ satisfy the same set of conditions as the rates $W$ except for the detailed balance condition which in the component space only holds at equilibrium. In general, rates $\mathcal{W}$ do not satisfy detailed balance so the FDT derived in the previous sections for the microscopic master equation will not be valid in the component space. They are, however, valid in equilibrium so, for example, after an appropriate redefinition of correlations and responses the equilibrium fluctuation-dissipation theorem (60) still holds in the component space.

\section{FDT extensions to the non-equilibrium regime}

In this section we discuss how to extend the previous ideas to the non-equilibrium glassy regime. After a brief introduction of aging (intended for the non-specialist) we discuss how to derive a free-energy master equation by introducing the configurational entropy and the notion of the effective temperature. We then discuss how to extend the FDT beyond equilibrium by introducing the fluctuation-dissipation ratio and the quasi-FDT. This requires the notion of neutral observables. Finally, a possible partitioning scheme is presented.

\subsection{An intermezzo on aging}

In this section we discuss one of the main signatures of non-equilibrium regime of glassy systems, i.e. the existence of aging and the quantifying of FDT violations through a modified FDT in terms of a set of effective temperatures. This discussion is intended for the inexpert reader who wants to have a glance on the key aspects of glassy systems before entering into the more detailed exposition. Therefore, the level of this discussion is highly introductory and the expert reader can move directly into the next section.

In relaxational glassy systems the two basic properties of correlations and response, time-translational invariance (TTI) 421) and the FDT (60), do not hold anymore. In particular, TTI is observed to be violated in the following way: both correlations and responses decay slower as the system gets older, i.e. the system is aging. This fact stems from several experimental observations in polymers and deformable materials [1, structural glasses [30] and spin glasses [13]. In driven systems the situation appears less complicated as TTI holds and only the FDT is violated. Some of the aspects described below carry over also to driven systems, however for sake of clarity we will stick in what follows to the case of aging systems.

Experimentally aging is manifest through the measurement of the so called integrated response function (IRF) or time-dependent susceptibility described in Section 4.3 In aging systems the weak long term memory makes response functions $R(t, s)$ hardly measurable as they asymptotically decay to zero, instead it is easier to measure the cumulative response or time-dependent susceptibility $\chi(t, s)=$ 
$\int_{s}^{t} R\left(t, t^{\prime}\right) d t^{\prime}$ (defined after perturbing the system at the waiting time $s$-often denoted as $\left.t_{w^{-}}\right)$which in general are finite: in dielectric measurements of glasses the IRF corresponds to the polarizability of the sample after cutting or applying an electric field; in magnetic systems it corresponds to the thermoremanent or zero-field cooled susceptibility; in mechanical systems aging is observed by measuring the deformation of the sample after applying a tensile load. All these measurements reveal that $\chi(t, s)$ is well approximated by the sum of two contributions,

$$
\chi(t, s)=\chi_{\mathrm{st}}(t-s)+\chi_{\mathrm{ag}}(t, s)
$$

where the first contribution $\chi_{\mathrm{st}}(t-s)$ stands for an stationary part that asymptotically decays to a finite value or plateau and $\chi_{\mathrm{ag}}(t, s)$ is the aging part that is well approximated by the scaling relation,

$$
\chi_{\mathrm{ag}}(t, s)=\widehat{\chi}\left(\frac{t}{s}\right)
$$

This scaling behavior, that is obtained within many solvable models, is known as full aging or simple $t / s$ scaling $\ddagger$. However, deviations from full aging have been reported in many experiments suggesting that this simple scaling behavior does not fully account for the experimental data. In particular, spin glass measurements clearly favour a subaging scenario where $\hat{\chi}$ has as scaling argument the variable $\frac{t}{s^{\delta}}$ with $\delta<1$ (we note, however, that experiments report values around 0.95 , i.e. very close to 1 ). The physical origin of these deviations is still unknown. For both correlation and response functions similar decompositions as in (70), (71) are expected to be valid but replacing $\chi$ by $C$ or $R$ (however, for the aging part of the response there is an additional factor $1 / t$ multiplying $\left.R_{\text {ag }}(t, s)\right)$. Correlations and responses are difficult to experimentally access. In theoretical or numerical simulation studies, the calculation of correlation functions is always preferred. Typical curves for the susceptibilities or correlations are depicted in Figure 2 Within the full aging scenario (71) it is usually shown that, in the asymptotic limit where both $s$ and $t$ are large, the FDT (60) is violated depending on the ratio $(t-s) / s$. If $(t-s) / s \ll 1, C(t, s) \simeq C_{\text {st }}(t-s)$ and FDT holds,

$$
\frac{\partial C_{\mathrm{st}}(t-s)}{\partial s}=T R_{\mathrm{st}}(t-s)
$$

However, in the other case $(t-s) / s \geq 1$, then $C(t, s) \simeq C_{\mathrm{ag}}(t, s)$ and the FDT (60) is violated according to the new relation,

$$
\frac{\partial C_{\mathrm{ag}}(t, s)}{\partial s}=T_{\mathrm{eff}}(s) R_{\mathrm{ag}}(t, s)
$$

where $T_{\text {eff }}(s)$ is a new parameter that enters into the new relation playing the role of an effective temperature. The particular way in which the FDT is violated suggests calling the new relation (73) a quasi fluctuation-dissipation theorem (QFDT). Although other possible terms have been used to refer to the modified FDT, here we will adhere to the term QFDT, as this is the one originally introduced by Horner [32 that expresses the idea of partial equilibration among a subset of degrees of freedom.

Aging carries associated the decomposition of time into time sectors. In the previous example of full aging (71) there are two time sectors depending on the ratio $(t-s) / s$ as described in (72), (73). However, as we already said, deviations from the full aging behavior are expected to be present in general. In those cases, the stationary

$\ddagger$ Coarsening system also deviate from the simple form (71) in favour of $\chi_{\mathrm{ag}}(t, s)=s^{-a} \widehat{\chi}(t / s)$ with $a \geq 0$. 


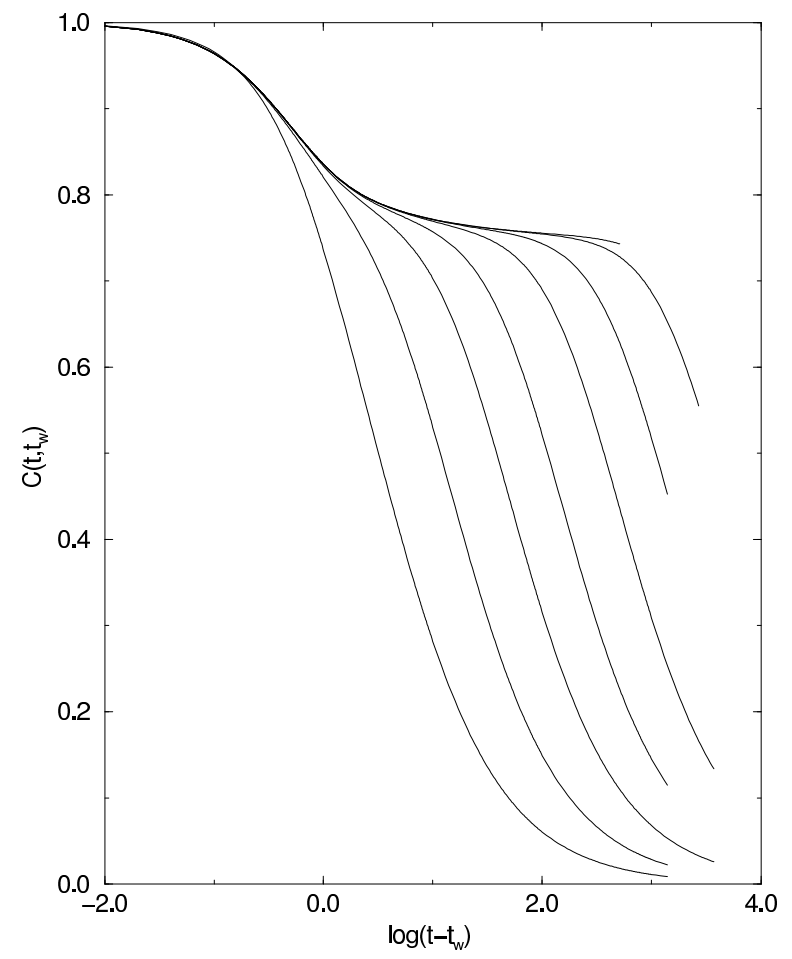

Figure 2. Typical shape of a two-time correlation function $C\left(t, t_{w}\right)$ (the same would be valid for the integrated susceptibility $\left.\chi\left(t, t_{w}\right)\right)$, plotted as a function of $t-t_{w}$ (in log-scale) with system age $t_{w}$ increasing from left to right. The decomposition (70) shows the two separate time sectors: in the first fast part of the relaxation $C\left(t, t_{w}\right) \simeq C_{\text {st }}\left(t-t_{w}\right)$ is independent of $s$ and obeys TTI while the slow second part $C_{\mathrm{ag}}\left(t-t_{w}\right)$ decays from the plateau in a timescale growing with $t_{w}$. This Figure has been taken from a lattice gas coarsening model 31 with properly normalized correlations at equal times $C(t, t)=1$.

result (72) for the short-time sector still holds but (73) is replaced by the more general relation,

$$
\frac{\partial C_{\mathrm{ag}}(t, s)}{\partial s}=T_{\text {eff }}\left(C_{\mathrm{ag}}(t, s)\right) R_{\mathrm{ag}}(t, s)
$$

where the new effective temperature $T_{\text {eff }}\left(C_{\text {ag }}(t, s)\right) \equiv T_{\text {eff }}(C(t, s))$, since $C \equiv C_{\text {ag }}$ when $(t-s) / s \sim O(1)$, and depends on both times only through the value of $C$. In this case, the aging part develops time sectors defined as those values of $t, s$ where $t / s \sim O(1)$ 33 34. Each sector is then labeled by the value of the correlation function $C(t, s)$ and many effective temperatures arise in the description of the non-equilibrium regime, the QFDT (74) quantifying FDT violations within each sector. Glassy systems are often classified into three different groups according to the dependence of $T_{\text {eff }}(C)$. For coarsening systems $T_{\text {eff }}(C)$ only takes two values: $T$ and infinite for the stationary and aging regimes respectively. $\S$. For structural glasses $T_{\text {eff }}(C)$ also takes two values: $T$ and $T_{\text {eff }}(s)>T$ for the stationary and aging regimes respectively. These are often referred as two-timescales systems. Finally for spin-glass systems $T_{\text {eff }}(C)$ takes a

$\S$ We note however that in some special cases coarsening systems can display more complex behaviour of $T_{\text {eff }}(C)$, see Sections 6.67.3 and the discussion in Section 13.1 in [3]. 
continuous spectrum of values extending from a lower bound $T^{*}>T$ up to infinity. These are known as many-timescales systems. All these three limit cases correspond to a well known static low-temperature description in the framework of spin-glass theory 35 in terms of replica-symmetry breaking (RSB): coarsening systems are those where replica symmetry (RS) is unbroken, structural glass systems correspond to one-step of RSB while spin-glass systems corresponds to full RSB. As a particular example of one-step systems there are some models (such as entropy barrier models, see section 6.5) that display glassy behavior only at zero temperature. The stationary regime is then absent in these models and their non-equilibrium dynamics is characterized by a single effective temperature, the bath temperature being zero. Along this review, we will often refer to them as one-timescale models. The three possible scenarios are depicted in figure 3 (see section 4.3 for a more detailed exposition). The experimental challenge of these ideas remains one of the most awaited results.

\subsection{The unbiased component ensemble and the master free energy equation}

One of the key ideas behind the existence of a quasi fluctuation-dissipation theorem (QFDT) in aging systems is the emergence of a non-equilibrium ensemble in the asymptotic long-time regime of the relaxation process. A related non-equilibrium ensemble could also emerge in driven stationary systems. Although the nature of this ensemble is yet to be understood we can anticipate some of its main properties. Some of these ideas have been already presented [36. Here we present more elaborated work.

In a description of glassy phenomena where the system is kept in contact with a thermal bath at temperature $T$ the energy is not constant and equipartition does not necessarily hold. Therefore this ensemble is neither microcanonical or canonical but of a more complicated nature.

The possible existence of a non-equilibrium ensemble traces back to Palmer 37. who introduced the unbiased component ensemble to characterize the equilibrium sampling of phase space components. Let us consider a given partition of phase space into components (see Section [3.3) and let us define the free energy $F(\mathcal{R})$ of a given component $\mathcal{R}$ by,

$$
F(\mathcal{R})=-T \log \left(\sum_{\mathcal{C} \in \mathcal{R}} \exp (-\beta \mathcal{H}(\mathcal{C}))\right)
$$

It is possible to extend this idea to the non-equilibrium regime by assuming an equal probability hypothesis: different components with identical free energy $F(\mathcal{R})$ do have the same probability,

$$
\mathcal{P}(\mathcal{R}, t)=\mathbf{P}(F(\mathcal{R}), t) .
$$

In what follows, we will use the letter $\mathcal{F}$ (as opposed to $F(\mathcal{R})$ ) to denote component free energies defined in (75) after dropping off the explicit argument $\mathcal{R}$. The existence of the unbiased ensemble tantamount to the appearance of a new measure based on free energy rather than on energy. Contrarily to Palmer [37] (who assumes an equilibrium probability distribution for $\mathbf{P}(\mathcal{F}, t)$, see (87) below) the probability distribution $\mathbf{P}(\mathcal{F}, t)$ is unknown, time dependent and must be found as a solution of a master equation (ME) as follows. To derive the free-energy ME we define the probability density,

$$
\mathbf{P}(\mathcal{F}, t)=\sum_{\mathcal{R}} \mathcal{P}(\mathcal{R}, t) \delta(\mathcal{F}-F(\mathcal{R}))=\mathcal{P}(\mathcal{F}, t) \Omega(\mathcal{F}, T)
$$


where we have used (76) and the definition,

$$
\Omega(\mathcal{F}, T)=\sum_{\mathcal{R}} \delta(\mathcal{F}-F(\mathcal{R}))
$$

and we have introduced explicitly the temperature dependence in $\Omega$ to stress the temperature dependence of the free energy (75). Although consistency requires to add the $T$ dependence also to $\mathcal{F}$ here we drop this dependence in order to lighten the notation. Equation (77) describes the probability for the system to be in a component of free energy $\mathcal{F}$ at time $t$. We have indicated it in bold to distinguish it from the probability $\mathcal{P}(\mathcal{R}, t)$. Summing (64) over components having identical free energy $\mathcal{F}$ we get,

$$
\frac{\partial \mathbf{P}(\mathcal{F}, t)}{\partial t}=\sum_{\mathcal{F}^{\prime}} \mathbf{P}\left(\mathcal{F}^{\prime}, t\right) Z_{t}\left(\mathcal{F} \mid \mathcal{F}^{\prime}\right)-\sum_{\mathcal{F}^{\prime}} \mathbf{P}(\mathcal{F}, \mathbf{t}) Z_{t}\left(\mathcal{F}^{\prime} \mid \mathcal{F}\right)
$$

with the conditioned probabilities $Z_{t}$ defined by,

$Z_{t}\left(\mathcal{F} \mid \mathcal{F}^{\prime}\right)=\frac{1}{\mathbf{P}\left(\mathcal{F}^{\prime}, t\right)} \sum_{\mathcal{R}, \mathcal{R}^{\prime}} \mathcal{W}\left(\mathcal{R} \mid \mathcal{R}^{\prime} ; t\right) \delta(\mathcal{F}(\mathcal{R})-\mathcal{F}) \delta\left(\mathcal{F}^{\prime}\left(\mathcal{R}^{\prime}\right)-\mathcal{F}^{\prime}\right) P\left(\mathcal{R}^{\prime}, t\right)$

where the $\mathcal{W}$ have been already defined in 676). Note that both $\mathcal{W}$ and $Z_{t}$ are time-dependent rates. Again, as for the transition probabilities [65), the new rates $Z_{t}\left(\mathcal{F} \mid \mathcal{F}^{\prime}\right)$ do not satisfy detailed balance but satisfy the other requirements (nonnegativeness, ergodicity and causality). Expression (80) is exact but intractable. As we are postulating the existence of the unbiased component ensemble (76), consistency in the component master equation (64) implies that the rate $\mathcal{W}\left(\mathcal{R}, \mathcal{R}^{\prime} ; t\right)$ is a timedependent function of the initial and final components, only through the value of their free-energies $\mathcal{F}\left(\mathcal{R}^{\prime}\right), \mathcal{F}(\mathcal{R})$,

$$
\mathcal{W}\left(\mathcal{R}, \mathcal{R}^{\prime} ; t\right)=\mathcal{W}_{t}\left(\mathcal{F}(\mathcal{R}), \mathcal{F}\left(\mathcal{R}^{\prime}\right)\right)
$$

The transition rates (80) can be further simplified,

$$
Z_{t}\left(\mathcal{F} \mid \mathcal{F}^{\prime}\right)=\mathcal{W}_{t}\left(\mathcal{F} \mid \mathcal{F}^{\prime}\right) \Omega(\mathcal{F}, T)
$$

The quantity $\Omega(\mathcal{F}, T)$ is exponentially large with the volume of the system and defines what we will denote as the configurational entropy or complexity $S_{c}(\mathcal{F}, T) \dagger$,

$$
\Omega(\mathcal{F}, T)=\exp \left(S_{c}(\mathcal{F}, T)\right)
$$

Therefore, all the information on the master equation (79) goes into the density of components $S_{c}(F, T)$ and the rates $\mathcal{W}_{t}\left(\mathcal{F} \mid \mathcal{F}^{\prime}\right)$. These contain all the information about the properties of the unbiased ensemble.

The description of glassy dynamics in terms of a master free-energy equation such as (79) has been wandering around for many years in the literature of the field. Several equations have appeared scattered in the literature during the last decades, but generally written in terms of the energy instead of the free energy, see for instance 38. These equations describe what are usually known as trap models (see Section [6.4). Examples master equations proposed by Dyre [39] and Bouchaud [40]. Other attempts include granular media [1].

$\dagger$ The term configurational entropy has often been used with different meanings, leading to confusion. Originally, as used by Adam and Gibbs for their thermodynamic theory, it denotes the part of the total entropy including only the configurational degrees of freedom. More recently, in the context of spin-glass theory, this concept has been coined to denote that part of the configurational entropy that counts the number of metastable states rather than configurations. It is with this last meaning that we understand it here. For a throughout discussion of this concept see section 5.1 
4.2.1. Complexity and the effective temperature Before closing the present discussion let us note that, in equilibrium, both the transition rates $Z_{t}\left(\mathcal{F} \mid \mathcal{F}^{\prime}\right)$ and $\mathcal{W}_{t}\left(\mathcal{F} \mid \mathcal{F}^{\prime}\right)$ are time-independent and satisfy detailed balance,

$$
\begin{aligned}
& \frac{Z^{\mathrm{eq}}\left(\mathcal{F}^{\prime} \mid \mathcal{F}\right)}{Z^{\mathrm{eq}}\left(\mathcal{F} \mid \mathcal{F}^{\prime}\right)}=\exp \left(-\beta\left(\Phi\left(\mathcal{F}^{\prime}, T\right)-\Phi(\mathcal{F}, T)\right)\right) \\
& \frac{\mathcal{W}^{\mathrm{eq}}\left(\mathcal{F}^{\prime} \mid \mathcal{F}\right)}{\mathcal{W}^{\mathrm{eq}}\left(\mathcal{F} \mid \mathcal{F}^{\prime}\right)}=\exp \left(-\beta\left(\mathcal{F}^{\prime}-\mathcal{F}\right)\right)
\end{aligned}
$$

with

$$
\Phi(\mathcal{F}, T)=\mathcal{F}-T S_{c}(\mathcal{F}, T)
$$

where $\Phi(\mathcal{F}, T)$ is a new potential where the free energy appears balanced by the complexity. The probability (77) assumes the simple form,

$$
\mathbf{P}^{\mathrm{eq}}(\mathcal{F})=\frac{\exp (-\beta \Phi(\mathcal{F}, T))}{\mathcal{Z}(\beta)}
$$

where $\mathcal{Z}(\beta)$ is given by (14). We will describe in Section 5.1.2 how this relation provides us with a tool to obtain the configurational entropy as function of both the free energy and the temperature.

The hints at the existence of this new potential were found by Kirkpatrick, Thirumalai and Wolyness 42, 43, 44 who identified the marginal transition $T_{\mathrm{A}}$ ("A" standing for activated) as the temperature below which different metastable states concur in such a number to compensate the lower equilibrium free energy of the paramagnetic or liquid state. The subindex in $T_{\mathrm{A}}$ stands for the fact that below that temperature activation is dominant and relaxation occurs in the form of activated jumps from one metastable state to another. $T_{\mathrm{A}}$ is identified with the mode-coupling transition temperature $T_{c}$ of mode-coupling theories (see section 6.1) and corresponds to a spinodal instability [42, 43, 44]. The mathematical argument behind the compensation of the free-energy of metastable states by the complexity is as follows. Let us decompose the canonical partition function of the system as a sum over a set of non-overlapping components $\mathcal{R}$ (as explained in section 3.31),

$$
\begin{aligned}
& \mathcal{Z}(T)=\sum_{\mathcal{C}} \exp (-\beta \mathcal{H}(\mathcal{C}))=\sum_{\mathcal{R}} \exp (-\beta \mathcal{F}(\mathcal{R})) \\
& =\sum_{\mathcal{F}} \Omega(\mathcal{F}, T) \exp (-\beta \mathcal{F})=\sum_{\mathcal{F}} \exp (-\beta \Phi(\mathcal{F}, T))
\end{aligned}
$$

where we have used the definition (75) for the free energy of components $\mathcal{F}(\mathcal{R})$ and (83), (86). Due to the extensive character of the variables $\mathcal{F}, S_{c}(\mathcal{F}, T)$ and $\Phi(\mathcal{F}, T)$, the dominant contribution to the sum in (88) is evaluated through the saddle point method. At each temperature $T$ there is a free energy $F^{*}(T)$ such that its contribution to the exponent (88) is dominant, i.e. $\mathcal{Z}(T) \sim \exp \left(-\beta \Phi^{*}(T)\right)$ where we have defined $\Phi^{*}(T)=\Phi\left(F^{*}(T), T\right)$. The behavior of this solution depends on the shape of the function $S_{c}(\mathcal{F}, T)$. In general this function is a monotonically increasing function of the free energy $\mathcal{F}$. Because the exponential is a positive definite function we have $\Phi(F, T) \geq F_{\text {para }}(T)$ where $\mathcal{Z}(T)=\exp \left(-\beta F_{\text {para }}(T)\right)$ and $F_{\text {para }}(T)$ denotes the paramagnetic free energy.

Above $T_{A}$ there is no solution $F^{*}(T)$ which can compensate the equilibrium paramagnetic free-energy and $\Phi(F, T)>F_{\text {para }}(T)$. Below $T_{A}$ a solution appears $F^{*}(T)$ 
such that $\Phi^{*}(T)=F_{\text {para }}(T)$ and gives the dominant contribution to (88) so $F^{*}(T)$ satisfies the saddle-point relation,

$$
\frac{1}{T}=\left.\frac{\partial S_{c}(\mathcal{F}, T)}{\partial \mathcal{F}}\right|_{\mathcal{F}=F^{*}(T)}
$$

The identity $\Phi^{*}(T)=F_{\text {para }}(T)$ implies $F_{\text {para }}(T)=F^{*}(T)-T S_{c}\left(F^{*}(T), T\right)$. This means that for $T \leq T_{\mathrm{c}}$ there is a band of components with free energy $F^{*}(T) \geq$ $F_{\text {para }}(T)$ (therefore with free energy above the equilibrium one) whose difference with $F_{\text {para }}(T)$ is compensated by the complexity $S^{*}(T)=S_{c}\left(F^{*}(T), T\right)$. This solution exists as long as $S^{*}(T)>0$. Because $S_{c}(F, T)$ is a monotonically increasing function of $F$ and both $F^{*}(T), S^{*}(T)$ decrease with $T$ there is a temperature $T_{\mathrm{K}}$ at which $S^{*}\left(T_{\mathrm{K}}\right)=0$. Below this temperature, the complexity vanishes and the solution $F^{*}(T)$ ceases to change with temperature (so equation (89) does not hold anymore) but sticks to its minimum value $F^{*}\left(T_{\mathrm{K}}\right)$. This is the entropy crisis scenario where $T_{\mathrm{K}}$ corresponds to the Kauzmann temperature [45].

In mean-field models it has been shown [46 that the complexity $S_{c}(F, T)$ defines a free-energy dependent effective temperature through the relation,

$$
\frac{1}{T_{\text {eff }}(\mathcal{F})}=\frac{\partial S_{c}(\mathcal{F}, T)}{\partial \mathcal{F}}
$$

From this relation, it emerges that the configurational entropy plays the role of the thermodynamic potential associated to the effective temperature. Similarly, the entropy is the potential conjugated to bath temperature in the microcanonical ensemble. However, (90) has been only derived in mean-field models close to the asymptotic free-energy threshold where lower free-energy states are inaccessible 46. 47]. In models that do not have a marginal free-energy threshold above the equilibrium value, it is possible to show that free-energies are uncorrelated random variables exponentially distributed [48, 35. The extension of these results beyond mean-field where all free energy states are accessible through activated processes is at the roots of the existence of the unbiased component ensemble. Some attempts have been proposed in [49, 50, 51]. In particular it would be very interesting to understand the general form of the transition probabilities $Z_{t}$ as these lead to very specific predictions amenable of numerical checks. The exponential character of the free-energy distribution of the lower free-energy states below the threshold, and the fact that this distribution is time dependent (as shown by the fact that $Z_{t}$ is itself time dependent) appear as the two crucial ingredients to understand the emergence of effective temperatures in glassy systems. These features are present in many models of glasses such as, mean-field spin-glass models, trap models (see Section 6.4) or entropy barrier models (see Section 6.5.1).

\subsection{The integrated response function (IRF) and fluctuation-dissipation (FD) plots}

When a system is in a non-equilibrium state its response to a external perturbation cannot be described, in general, by FDT relations such as (60) since this has been derived assuming that the system is in a stationary state. However, the glassy state is a particular non-equilibrium state characterized by extremely slow relaxation processes. Hence, while the system is not in a real equilibrium state, it may be thought as being in a sort of quasi-equilibrium regime over time-scales much longer than the microscopic time-scales but still smaller than the typical relaxation time-scales of 
the slow processes. In this quasi-equilibrium regime the evolution of the system is quasi-stationary since non-stationary effects are seen only for times of the order of the time-scales of the slow processes. In this situation we may think that relations similar to (60) can still be valid, even if TTI cannot be assumed anymore. Thus a possible generalization of FDT to the glassy regime requires to introduce the following nondimensional quantity:

$$
X_{A, B}(t, s)=\frac{T R_{A, B}(t, s)}{\frac{\partial}{\partial s} C_{A, B}(t, s)}, \quad t>s
$$

where $X_{A, B}(t, s)$ is called the Fluctuation-Dissipation Ratio (FDR). In equilibrium $X_{A, B}(t, s)=1$ whatever times $t, s$ and observables $A, B$ are used [Cfr. [60)]. Thus $X$ is a measure of the violation of the true equilibrium in the quasi-equilibrium glassy state. The validity of (91), i.e. the proportionality between the response and the time derivative of correlation function, can only be checked a posteriori since it is based on the quasi-equilibrium hypothesis that up to now it has been proved only in mean-field models. Eliminating one time in favour of the correlation function, the time dependence of $X_{A, B}(t, s)$ can be recast in the form

$$
X_{A, B}(t, s) \equiv X_{A, B}\left[C_{A, B}(t, s), s\right] .
$$

The FDR was first studied in spin glasses where analytical results have shown that glassy systems in general satisfy the weak ergodicity breaking scenario [40, discussed in Section 6.2 For the present purpose it is enough to note that calculations in mean-field spin-glass models [52, 53] have shown that in the limit $s \rightarrow \infty$ the FDR is a non-trivial function which depends on the relation between the times $t$ and $s$ only through the correlation function $C_{A, B}(t, s)$. The following specific form of FDT violations has been proposed to be generically valid in the non-equilibrium regime of glassy systems,

$$
\lim _{s \rightarrow \infty} X_{A, B}(t, s)=X_{A, B}\left[C_{A, B}(t, s)\right]
$$

Using (93) and (91) we obtain the differential form of the Quasi-FDT (QFDT) relation [32,

$$
X_{A, B}(C)=\left[\frac{T R_{A, B}(t, s)}{\frac{\partial}{\partial s} C_{A, B}(t, s)}\right]_{C_{A, B}(t, s)=C} t>s .
$$

which describes the response of the system in the (quasi)-equilibrium state to a impulsive perturbation at time $s<t$. Experiments and numerical simulations usually measure integrated response functions $\dagger$ (IRF), i.e., the response at time $t$ to a perturbation switched on or off at time $s<t$. According to the definition (43) the variation of the observable $\langle A(t)\rangle_{\epsilon}$ to linear order in the perturbation intensity $\epsilon$ is given by,

$$
\langle A(t)\rangle_{\epsilon_{s}}=\langle A(t)\rangle_{0}+\epsilon R_{A, B}(t, s)+\mathcal{O}\left(\epsilon^{2}\right), \quad t>s .
$$

Assuming that the perturbation acts for all times $t>s$ and that its intensity is small enough for the accumulated response to be linear in $\epsilon$ we get

$$
\begin{aligned}
\langle A(t)\rangle_{\epsilon_{s}} & =\langle A(t)\rangle_{0}+\sum_{t^{\prime}=s}^{t} \epsilon_{t^{\prime}} R_{A, B}\left(t, t^{\prime}\right) \\
& =\langle A(t)\rangle_{0}+\int_{s}^{t} d t^{\prime} \epsilon\left(t^{\prime}\right) R_{A, B}\left(t, t^{\prime}\right)
\end{aligned}
$$

$\dagger$ The integrated response functions are also called time dependent or non-equilibrium susceptibilities. 
where $\epsilon_{t^{\prime}}\left(\right.$ or $\left.\epsilon\left(t^{\prime}\right)\right)$ is the perturbation at time $t^{\prime}$. In the particular case of $\epsilon\left(t^{\prime}\right)=$ $\epsilon \theta\left(t^{\prime}-s\right)$, i.e., of a constant perturbation, one gets

$$
\chi_{A, B}(t, s)=\lim _{\epsilon \rightarrow 0} \frac{\langle A(t)\rangle_{\epsilon_{s}}-\langle A(t)\rangle_{0}}{\epsilon}=\int_{s}^{t} d t^{\prime} R_{A, B}\left(t, t^{\prime}\right) .
$$

which is also called zero-field cooled (ZFC) susceptibility. The name zero-field cooled follows from the experimental protocol used in spin glass measurements to distinguish it from the thermoremanent magnetization (TRM). To measure the TRM susceptibility a constant external perturbation is applied to the system at time $t=0$ and removed at time $s>0$ and the subsequent decay of $\langle A(t)\rangle$ is recorded. The TRM susceptibility is given by

$$
\chi_{A, B}^{\mathrm{TRM}}(t, s)=\lim _{\epsilon \rightarrow 0} \frac{\langle A(t)\rangle_{\epsilon_{s}}-\langle A(t)\rangle_{0}}{\epsilon}=\int_{0}^{s} d t^{\prime} R_{A, B}\left(t, t^{\prime}\right)
$$

In the large $t, s$ limit the ZFC (97) and TRM (98) susceptibilities are equivalent since they are related by,

$$
\chi_{A, B}(t, s)+\chi_{A, B}^{\mathrm{TRM}}(t, s)=\chi_{A, B}(t, 0)
$$

and $\chi_{A, B}(t \rightarrow \infty, 0)=\chi_{A, B}^{\mathrm{eq}}$.

Inserting the QFDT relation (94) in (97) we obtain the formula

$$
\chi_{A, B}(t, s)=\frac{1}{T} \int_{C_{A, B}(t, s)}^{C_{A, B}(t, t)} d C^{\prime} X_{A, B}\left(C^{\prime}\right)
$$

which relates $\chi_{A, B}(t, s)$ to the FDR $X_{A, B}$ and provides a simple way to calculate $X_{A, B}$ from measurements of $C_{A, B}(t, s)$ and $\chi_{A, B}(t, s)$ in the time sector $t>s$. Suppose indeed we fix the lowest time $s$ and plot $\chi_{A, B}(t, s)$ as function of $C_{A, B}(t, s)$ for different values of $t$, then the value of the FDR can be obtained from the slope of the resulting curve. In many of the examples considered in this review the equal times correlation function is time-independent, for instance $C_{A, B}(t, t)=1$. In this case the slope can be simply obtained by derivation of $\chi_{A, B}(t, s)$ with respect to $C_{A, B}(t, s)$ for fixed $s$ which from (100) yields,

$$
X_{A, B}(C)=-\left.\beta \frac{\partial \chi_{A, B}(t, s)}{\partial C_{A, B}(t, s)}\right|_{C_{A, B}(t, t)=\text { const, } s \text { fixed }}
$$

Typical FD plots are shown in Figure 3 for the three possible scenarios (see section 4.1). In the general case in which $C_{A, B}(t, t)$ is time-dependent one needs to be more careful in computing the FDR. Sollich and coworkers have proposed [54 [55] to construct FD plots $\chi$ versus $C$ with $t$ kept constant and varying the lowest time $s$. From (91) we have,

$$
X_{A, B}(C)=-\left.\beta \frac{\partial \chi_{A, B}(t, s)}{\partial C_{A, B}(t, s)}\right|_{t \text { fixed }}
$$

If $C(t, t)$ changes with time it is convenient to normalize correlations and the IRF by the equal times correlation $C_{A, B}(t, t)$ :

$$
\widetilde{C}_{A, B}(t, s)=\frac{C_{A, B}(t, s)}{C_{A, B}(t, t)}, \quad \widetilde{\chi}_{A, B}(t, s)=\frac{\chi_{A, B}(t, s)}{C_{A, B}(t, t)} .
$$

With these definitions,

$$
X_{A, B}(C)=-\left.\beta \frac{\partial \widetilde{\chi}_{A, B}(t, s)}{\partial \widetilde{C}_{A, B}(t, s)}\right|_{t \text { fixed }}
$$




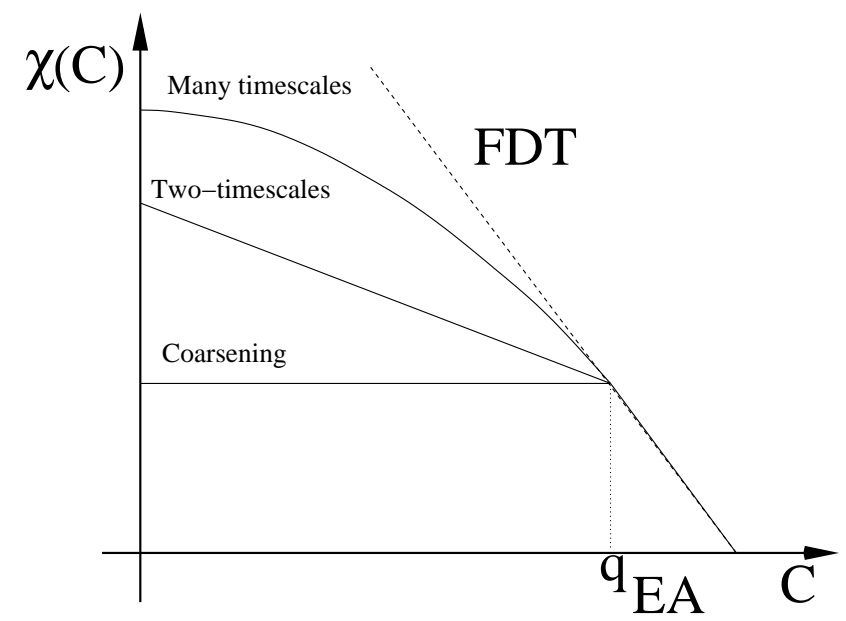

Figure 3. FD plots for the three possible scenarios. From bottom to top: 1) RS models or coarsening systems with two-timescales and two-temperatures (one identical to the bath, the other infinite), 2) one-step RSB models with two-timescales and two-temperatures (one identical to the bath, the other finite but higher than that of the bath), 3) full RSB models with many timescales and temperatures. For $C>q_{\mathrm{EA}}$ (the Edwards-Anderson parameter), i.e. the stationary regime, all models satisfy FDT (the dashed line).

The importance of normalizing the raw FD plots (102) is well appreciated in trap models discussed in section 6.4 or in kinetically constrained models discussed in section 7.5] In this last case, for example, raw FD plots can lead to awkward representations as the one shown in Figure 36.

\subsection{The concept of neutral observables}

If the FDR (91), (94) has the physical interpretation of a temperature (as has been suggested, see the discussion in Section (5.2) then one would expect the FDR to be independent on the observables $A, B$ used to construct correlations and responses. In fact, this is true in equilibrium where $X_{A, B}=1$ whatever $A, B$. However, although $X_{A, B} \neq 1$ observable independence is not at all required in the glassy regime. In this section we present a brief digression on which conditions the observables $A, B$ must satisfy for the FDR to be observable independent. This issue is yet unresolved, so the present discussion is quite speculative.

Albeit restricted, for simplicity we will consider here the case of a glassy system with only two-timescales where $A=B$ (so we will denote $X_{A, A}$ simply by $X_{A}$ ) in the time sector where $(t-s) / s \sim \mathcal{O}(1)$ or $X_{A} \neq 1$. In equilibrium one could argue that the equality $X_{A}=1$ is related to the fact that the entropy $S(E, A)$, as defined in the microcanonical ensemble Section 2.1] is maximum (12) for $A$ equal to its equilibrium value. This property, is observable independent as well as it is the identity $X_{A}=1$. A similar argument, but extended to the glassy regime, would require to define the configurational entropy $S_{c}(\mathcal{F}, \mathcal{A})$ (i.e. the equivalent generalization (2) of (83)) where $\mathcal{F}, \mathcal{A}$ denote the component averaged values of the free-energy (75) and the corresponding restricted Gibbs average for the observable $A$. We could then say that $A$ is a neutral observable if its dynamically averaged value at all times $<A(t)>$ 


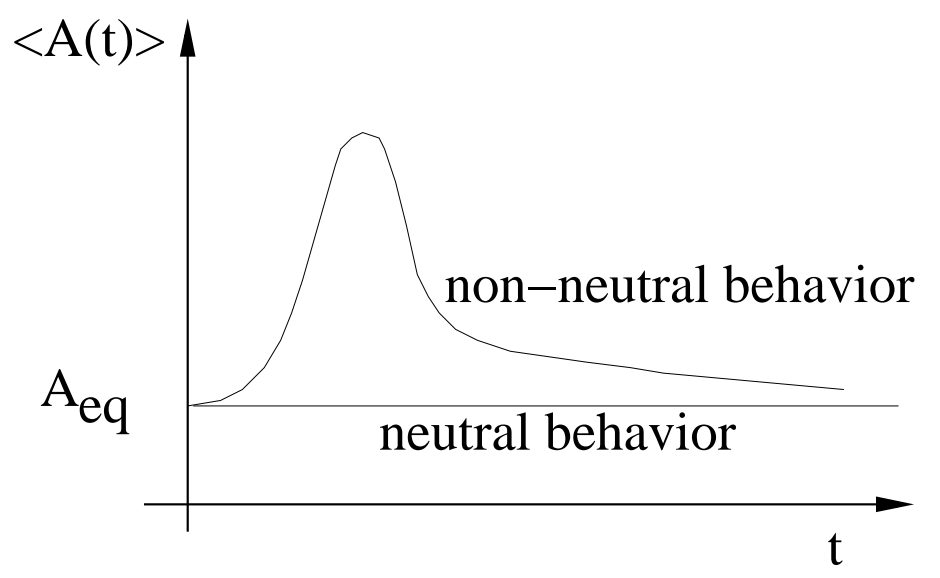

Figure 4. Illustration of the neutrality property of observables. The value of a neutral observable remains fixed to its initial (equilibrium) value despite of the fact that the initial state is non-equilibrium.

coincides with the stationary maximum of the function $S_{c}(\mathcal{F}, \mathcal{A})$ as $\mathcal{A}$ is varied. Of course, if this were not true the value of $X_{A}$ would then depend on the value of $A$ in the same way that the value of the temperature $1 / T=\beta$ in the microcanonical ensemble, and for a given value of the energy $E$, would depend on the value of the observable $A$ if $\mu$ in (83) were not zero.

For instance, the magnetization in mean-field spin-glass models is known to be a neutral observable and nearly all computations of the FDR have used this observable (see Sections 6.2). In fact, in the framework of the TAP approach it can be shown that the configurational entropy evaluated as a function of the free-energy and magnetization of the TAP states is maximum at zero magnetization. Indeed, the fact that the magnetization is not a good order parameter in these models (it vanishes in both the paramagnetic and the spin-glass phase) is related to its neutral character. Not by chance the majority of numerical studies in glassy systems use the magnetization as the central observable to investigate and measure FDT violations.

A non-neutral observable $A$ would correspond to a situation where, starting at time zero from a non-equilibrium initial state where the value of $A$ is taken to coincide with its equilibrium value (this is not a contradiction, since $A$ could coincide with its equilibrium value but not the value of any other observable) the subsequent evolution of $A$ deviates from its initial equilibrium value. In this case $A$ is not neutral because its time evolution is correlated to that of the other observables. On the contrary, if $A$ were a neutral observable, then it would stick to the value $A$ forever (as happens for the magnetization in mean-field spin-glass models). Fig团illustrates this behavior. The definition of neutrality can be easily adapted in trap models by assuming that observable values of traps are uncorrelated with their energies [54] (see also Section 6.4).

\subsection{Numerical approach to component dynamics: Stillinger-Weber decomposition}

The increase of computational power and the recent developments in the theory of disordered systems has pushed forward an approach to the glass transition based on the analysis of a reduced dynamics in the component space. The underlying ideas date 
back to more than 30 years ago to a seminal, but talkative, paper of Goldstein [56]. The glass transition is of purely dynamical origin and hence must reflect the properties of the dynamical evolution of the system. Goldstein suggested that the dynamics of a supercooled liquid can be understood in terms of a diffusive process between different basins of the potential energy surface. At low temperature the dynamics slows down since the system gets trapped for a long time in a basin. This approach, which focus on the topological properties of the energy surface is rather appealing since it naturally leads to a convenient framework for understanding the complex phenomenology of glassy systems.

The implementation of these (qualitative) ideas came some years later by Stillinger and Weber [57, 58, 59] (SW) who formalized the concept of basin in configuration space identifying it with a component in the component space, and proposed a procedure for identifying them: the set of all configurations connected to the same local energy minimum by a steepest-descent path on the energy surface uniquely defines the basins of the minimum. Stillinger and Weber (SW) called the local minimum inherent structure (IS) to stress its intrinsic nature. Since the identification of ISs is unique, the mapping from configurations to local minima gives a unique well defined decomposition of the phase space into a disjoint set of basins. The SW decomposition defines a mapping of the phase space to the component space in which each basin, usually labeled by the energy $E_{\mathrm{IS}}$ of the local minimum, is a component.

This decomposition does not cover completely the configuration space since it leaves out the boundaries between different basins. However, under the assumption that those configurations do non contribute to the thermodynamic of the system, e.g., the boundaries between basins are sub-extensive, it does cover almost all the phase space and the partition function can be written as a sum of contributions from different components:

$$
\mathcal{Z}(T) \simeq \sum_{E_{\mathrm{IS}}} \mathcal{Z}_{\mathrm{IS}}\left(E_{\mathrm{IS}}, T\right)
$$

Let $\Omega(E)$ denote the number of IS with energy $E_{\mathrm{IS}}=E$, then collecting all components with the same value of $E_{\mathrm{IS}}$

$$
\begin{aligned}
\mathcal{Z}(T) & \simeq \sum_{E} \Omega(E) \sum_{E_{\mathrm{IS}}=E} \mathcal{Z}_{\mathrm{IS}}(T) / \Omega(E) \\
& =\sum_{E} \exp \left[S_{c}(E)-\beta F_{b}(T, E)\right] .
\end{aligned}
$$

The term

$$
S_{c}(E)=\log \Omega(E),
$$

which accounts from the entropic contribution arising from the number of different basins with the same IS energy, is called the SW configurational entropy of complexity. This quantity is strongly related to the partitioning, so we add the adjective SW to distinguish it to other definitions of configurational entropy taken from mean-field concepts.

The second term $F_{b}(T, E)$ is defined as

$$
F_{b}(T, E)=-T \log \left[\frac{1}{\Omega(E)} \sum_{E_{\mathrm{IS}}=E} \mathcal{Z}_{\mathrm{IS}}(T)\right] .
$$

In general this quantity differs from the average free energy of components with $E_{\mathrm{IS}}=$ $E$, however if all these components have similar statistical properties, then $F_{b}(T, E)$ 
is the free energy of the system when constrained to any one of the components with $E_{\mathrm{IS}}=E$. In the thermodynamic limit the system populates components with energy $E_{\mathrm{IS}}=E_{\mathrm{IS}}(T)$ fixed by the condition

$$
-\beta F(E)=S_{c}(E)-\beta F_{b}(T, E)=\text { maximum over } E
$$

and the free energy of the system can be calculated using

$$
F(T)=F_{b}\left[T, E_{\mathrm{IS}}(T)\right]-T S_{c}\left[E_{\mathrm{IS}}(T)\right]
$$

The condition (109) is equivalent to that of $F(T)$ being minimal, i.e.,

$$
\frac{\partial F}{\partial E}=\frac{\partial F_{b}(T, E)}{\partial E}-T \frac{\partial S_{c}(E)}{\partial E}=0 .
$$

Note that the minimum condition follows from the balance between the contribution from the change with the energy of the shape of the basins $\left(\partial F_{b}(T, E) / \partial E\right)$ and its corresponding number $\left(\partial S_{c}(E) / \partial E\right)$. Often the free energy is written as

$$
F_{b}\left(T, E_{\mathrm{IS}}\right)=E_{\mathrm{IS}}+F_{v}\left(T, E_{\mathrm{IS}}\right)
$$

The first term in (112) takes into account the average energy of IS visited in equilibrium at temperature $T$, as can be seen from (110): $U(T)=\partial\left(\beta F_{b}\right) / \partial \beta=$ $E_{\mathrm{IS}}(T)+\partial\left(\beta F_{v}\right) / \partial \beta$. It can be shown 60, 61 that if the density of states $\Omega(E)$ is Gaussian and the basins have approximately the same shape then $E_{\mathrm{IS}} \propto 1 / T$. The second term in (112) describes the volume of the corresponding components and is called the "vibrational" contribution.

To understand the success and limitation of the IS approach we have to analyze the idea behind the SW approach. It is clear that even if the phase space can be always partitioned, not all possible partitions will lead to a physically relevant dynamics in the component space. This is a well known problem in the theory of dynamical systems, where the component dynamics is called symbolic dynamics, see e.g. Ref. [22]. To prove that the SW is a physically good partition for a given system is a problem of the same hardness as proving ergodicity. One then adopts a constructive point of view, along the same lines of equilibrium statistical theory: based on some reasonable hypothesis one first assumes that the SW partition is a good partition and then check if this reproduces the desired features of the dynamics.

The physical motivation behind the SW proposal follows from the observation that the potential energy surface of a super-cooled liquid contains a large number of local minima and that the time evolution can be separated into two different processes: thermal relaxation into basins (intra-basin motion) and thermally activated potential energy barrier crossing between different basins (inter-basin motion). This scenario has been recently confirmed from numerical analysis 60, 61, 62, 63, 64. The timescales separation of the two processes strongly depends on temperature. When the temperature is lowered down to the order of the critical mode-coupling theory (MCT) temperature $T_{\mathrm{c}}$ the typical barrier height is of the order of the thermal energy $k_{B} T_{\mathrm{c}}$, and the slow inter-basin motion dominates the relaxation dynamics. If the temperature is further reduced the relaxation time eventually becomes of the same order of the physical observation time and the system falls to a non-equilibrium state since there is not enough time to cross barriers and equilibrate. With this picture in mind it is natural to view the IS partitioning as the natural elements to describe the slow glassy dynamics. This approach is rather appealing since naturally leads to universality: all glassy systems with similar IS dynamics must have similar glassy behaviour. Recent IS analysis performed on disordered spin systems displaying a transition of fragile 
glass type do support this conclusion [65, 66, 67, 68, 36]. It should be noted 69] that the definition of IS for spin systems is more subtle than for systems with continuous variables. Indeed usually for spin systems IS are defined as one-spin flip stable states, however these may be not stable for two-spin or higher number of spin flips. One possibility of making IS well defined also for spin systems is to define them directly from the $T=0$ limit of the dynamics, i.e., as states which are stable under the $T=0$ dynamics [70]. This is the definition used in this review when discussing IS for spin systems.

\section{Thermodynamic description of the aging state}

We saw in section 4.2 how the self-generated dynamical measure allows a description of the aging dynamics in terms of a probabilistic master equation with transition rates characterized by an extensive quantity that was defined as a configurational entropy or complexity (83). This quantity has received considerable attention in studies of spin-glasses since the seminal paper of Thouless, Anderson and Palmer (TAP) [71] on the SK model where a way to compute the configurational entropy was proposed 72. Later studies in the context of structural glasses [42, 43, 44] have shown its importance as the mechanism for an entropy crisis of the supercooled liquid as proposed by Kauzmann many years ago [45].

\subsection{Methods to compute the complexity}

In this section we present a schematic overview of some of the analytical and numerical methods that have appeared in the literature to compute the configurational entropy. In the absence of a full solution of the dynamics in many systems, and under the assumption that there is a connection between the effective temperature and the configurational entropy (see the discussion in Section 4.2.1), the calculation of the later, by using equilibrium methods taken from statistical physics, appears as an alternative way of quantifying FDT violations. In mean-field theories metastable states give a natural partition of the phase space since their life-time diverges in the thermodynamic limit. For systems with short-range interactions, however, metastable states can be defined unambiguously only referring to some reference time-scale. Therefore the identification of metastable states for real systems can be a very hard task. In section 4.5 we have presented a partition scheme, proposed by Stillinger and Weber, which in principle can be applied to any system. The scheme essentially uses a zero-temperature dynamics and thus it is free from the ambiguities due to the finite metastable life-time. The results described in next sections must be seen as instructive attempts to evaluate a quantity (the complexity) that governs the slow dynamics of relaxational glassy systems. The extension of these equilibrium concepts to other non-equilibrium systems beyond aging systems (e.g. driven systems) remains an open problem.

5.1.1. Analytical methods. Bray and Moore [72] calculated $S_{c}(\mathcal{F}, T)$ for the Sherrington-Kirkpatrick model within the TAP approach. The TAP equations give the local magnetization $m_{i}$ in a system confined to a metastable state, which for mean-field models have infinite life-time. As a consequence the number of metastable states (i.e., components) can be readily obtained just counting the number $\mathcal{N}_{s}(\mathcal{F}, T)$ 
of solutions of the TAP equations at temperature $T$ with a free energy $\mathcal{F}$ :

$$
\begin{aligned}
\mathcal{N}_{s}(\mathcal{F}, T)=\int_{-1}^{1} \prod_{i=1}^{N} d m_{i} \mid & \operatorname{det} H\left(\left\{m_{i}\right\}\right) \mid \\
& \times \delta\left(\mathcal{F}-\mathcal{F}\left(\left\{m_{i}\right\}, T\right)\right) \delta\left(g_{i}\left(\left\{m_{i}\right\}\right)\right)
\end{aligned}
$$

where $\mathcal{F}\left(\left\{m_{i}\right\}, T\right)$ if the TAP free energy at temperature $T$ as function of the local magnetizations $m_{i}, g_{i}\left(\left\{m_{i}\right\}\right)=\partial \mathcal{F}\left(\left\{m_{i}\right\}, T\right) / \partial m_{i}=0$ are the TAP equations and $H_{i j}=\partial g_{i}\left(\left\{m_{i}\right\}\right) / \partial m_{j}$ the Hessian. This type of calculation has been done for other mean-field models, such as $p$-spin [73] and Random Orthogonal Model (ROM) [74, 75]), finding in all cases that $\mathcal{N}_{s}(\mathcal{F}, T)$ increases exponentially fast with the system size system $N$. This remains true if the number of free-energy minima $\mathcal{N}_{m}(\mathcal{F}, T)$, instead of the number of stationary points $\mathcal{N}_{s}(\mathcal{F}, T)$, is considered [76]. Although these type of calculations can be done only in exactly solvable mean-field models the exponential growth with the system size of the number of free energy local minima or stationary points is generally applicable to any system (mean-field or not) displaying glassy behaviour. The knowledge of the number of minima allows to define the complexity (78) as,

$$
S_{c}(\mathcal{F}, T)=\log \mathcal{N}_{m}(\mathcal{F}, T)
$$

and hence the thermodynamic potential $\Phi(\mathcal{F}, T)=\mathcal{F}-T S_{c}(\mathcal{F}, T)$ as described in section 4.2

A general framework to evaluate the complexity has been devised by Monasson 77. The starting point in his procedure is to consider $m$ interacting copies or replicas of the original system, with an attractive interaction term of the form $\epsilon \sum_{a, b=1}^{m} Q\left(\mathcal{C}_{a}, \mathcal{C}_{b}\right)$ where $Q\left(\mathcal{C}_{a}, \mathcal{C}_{b}\right)$ is a suitable overlap function which takes its maximum value only if $\mathcal{C}_{a}=\mathcal{C}_{b}$. The free energy of the replicated system is then,

$$
e^{-\beta F(T, m)}=\sum_{\mathcal{C}_{1}, \ldots, \mathcal{C}_{m}} \exp \left[-\beta \sum_{a=1}^{m} \mathcal{H}\left(\mathcal{C}_{a}\right)+\beta \epsilon \sum_{a, b=1}^{m} Q\left(\mathcal{C}_{a}, \mathcal{C}_{b}\right)\right]
$$

If the thermodynamic limit is taken before the limit $\epsilon \rightarrow 0^{+}$then the configurations $\mathcal{C}_{a}, \mathcal{C}_{b}$ tend to lie as close as possible since maximization of the coupling term minimize the global free energy $F^{(m)}(T)$ and hence, given a phase space partition, the replicas tend to "condensate" into the same component. Thus collecting all components with the same free energy the partition function $\mathcal{Z}(T, m)$ can be decomposed as

$$
\begin{aligned}
\mathcal{Z}(T, m) & =\sum_{\mathcal{F}} \Omega(\mathcal{F}, T) \exp (-m \beta \mathcal{F}) \\
& =\sum_{\mathcal{F}} \exp [-\beta \Phi(\mathcal{F}, T, m)]
\end{aligned}
$$

where $\Phi(\mathcal{F}, T, m)=m \mathcal{F}-T S_{c}(\mathcal{F}, T)$ is basically the potential $\Phi(\mathcal{F}, T)$ discussed in section 4.2 with the term $\mathcal{F}$ multiplied by $m$ (the order of limits, first volume $\rightarrow \infty$ and then $\epsilon \rightarrow 0^{+}$, enforces the $m$ replicas to occupy the same component $\left.\mathcal{R}\right)$. In the limit $m \rightarrow 1$ we recover the potential $\Phi(\mathcal{F}, T): \Phi(\mathcal{F}, T, m=1)=\Phi(\mathcal{F}, T)$. The knowledge of $\Phi(\mathcal{F}, T, m)$ allows to compute the configurational entropy. In the thermodynamic limit the sum in (116) is dominated by the free energy $F^{*}(T, m)$ that satisfies the relation,

$$
\frac{m}{T}=\left.\frac{\partial S_{c}(\mathcal{F}, T)}{\partial \mathcal{F}}\right|_{\mathcal{F}=F^{*}(T, m)}
$$


Inserting the solution $F^{*}(T, m)$ into $\Phi(\mathcal{F}, T, m)$ we obtain the free-energy potential $\Phi^{*}(T, m)=\Phi\left(F^{*}(T, m), T, m\right)$. If $m$ (originally an integer value) is continued to real values then it can be show that the following relations are satsified,

$$
\begin{aligned}
& \frac{\partial}{\partial m} \Phi^{*}(T, m)=F^{*}(T, m), \\
& \frac{\partial}{\partial m}\left[\frac{\Phi^{*}(T, m)}{m}\right]=\frac{T}{m^{2}} S_{c}\left(F^{*}, T\right) .
\end{aligned}
$$

Varying $m$ allows to compute the configurational entropy $S_{c}(\mathcal{F}, T)$ as function of the two variables $\mathcal{F}$ and $T$. The potential $\Phi(\mathcal{F}, T, m)$ can be explicitly evaluated with the sole knowledge of the microscopic Hamiltonian of the system and using the replica method. Although this procedure was initially applied only to mean-field disordered systems [77, 78], more recently it has been extended to more realistic interacting potentials such as Lennard-Jones liquids [79, 80, and binary mixtures [81, 82.

This method of computing the configurational entropy can be easily implemented in the framework of the standard replica method for mean-field disordered systems. This has been worked in some detail in [83, 84]. The starting point is free energy at one-step level of replica symmetry breaking $F\left(q_{0}, q_{1}, m\right)$, where $q_{0}, q_{1}$ and $m$ are the parameters that describe the Parisi matrix [35] in the one-step replica symmetry breaking scheme. By expanding the free energy around $m=1$ one gets, $F\left(q_{0}, q_{1}, m\right)=F_{R S}\left(q_{0}\right)+F^{(1)}\left(q_{0}, q_{1}\right)(m-1)+\mathcal{O}\left((m-1)^{2}\right)$ where $q_{0}$ stands for the overlap among replicas belonging to different subboxes and $F_{R S}\left(q_{0}\right)$ is the free energy in the replica symmetric approximation, i.e., in the limit $m \rightarrow 1$. Extremization of $F_{R S}\left(q_{0}\right)$ yields $q_{0}(\beta)$ which inserted into $F\left(q_{0}, q_{1}, m\right)$ allows to find $F_{R S}(\beta), F^{(1)}\left(\beta, q_{1}\right)$. The knowledge of these functions fully determines the configurational entropy of the system for temperature $T_{\mathrm{RSB}}<T<T_{\mathrm{c}}$ (where $T_{\mathrm{c}}$ corresponds to the MCT, see Section [6.1). Indeed the dynamical transition $T_{\mathrm{c}}$ is found solving the equations $\left(\partial / \partial q_{1}\right) F^{(1)}\left(\beta, q_{1}\right)=\left(\partial^{2} / \partial q_{1}^{2}\right) F^{(1)}\left(\beta, q_{1}\right)=0$ while the static transition $T_{\mathrm{RSB}}$, where the configurational entropy vanishes, follows from the solution of $F^{(1)}\left(\beta, q_{1}\right)=\left(\partial / \partial q_{1}\right) F^{(1)}\left(\beta, q_{1}\right)=0$. Finally the complexity in the region $T_{\mathrm{RSB}}<T<T_{\mathrm{c}}$ is given by the value of $F^{(1)}\left(\beta, q_{1}\right)$ evaluated for $q_{1}(\beta)$ solution of the equation $\left(\partial / \partial q_{1}\right) F^{(1)}\left(\beta, q_{1}\right)=0$. This approach gives a detailed description of the metastable properties in the range $T_{\mathrm{RSB}}<T<T_{\mathrm{c}}$. Below $T_{\mathrm{RSB}}$ more sophisticated methods are needed to describe the metastable behavior.

The potential method has been proposed by Franz and Parisi 85$]$ in the framework of the replica approach. The starting point in this procedure is to write down the partition function of a generic system at temperature $T$ whose configurations $\mathcal{C}$ are constrained to have an overlap $Q\left(\mathcal{C}, \mathcal{C}_{0}\right)$ with a reference configuration $\mathcal{C}_{0}$. The free energy of the constrained system is then averaged, using the replica method, over the reference configuration $\mathcal{C}_{0}$ thermalized at a temperature $T^{\prime}$ in general different from $T$. This yields the potential $V\left(Q, T, T^{\prime}\right)$. For $T=T^{\prime}$, and in a given range of temperatures, the potential $V$, as a function of $Q$, has two local minima. The difference of the potential at these two values yields the configurational entropy at that temperature. The method has been applied to evaluate the configurational entropy in the hypernetted chain approximation usually employed for liquids 86, 87.

5.1.2. Numerical methods Among numerical approaches, Speedy [88 has proposed a method that consists in estimating what he defines as the statistical entropy (basically identical to the intrastate entropy in the inherent-structures approach discussed 


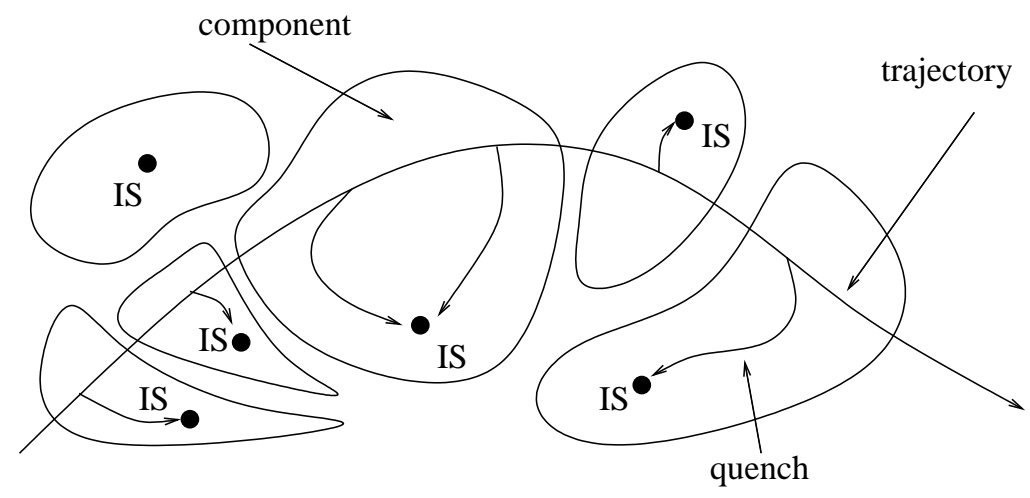

Figure 5. Stillinger and Weber decomposition

in section 4.5) and comparing it to the thermal or total entropy obtained from integration of the specific heat. The difference between the thermal entropy and the statistical entropy is the complexity. To compute the statistical entropy the method considers different reference configurations representative of an amorphous glass state and introduces a coupling term between a reference configuration and the system that forces it to stay within a given distance of that reference configuration. By progressively slowly changing the intensity of the coupling the energy of the system can be evaluated for each value of the coupling. The entropy associated to a particular reference state is then estimated by integrating the energy as function of the intensity of the coupling. Speedy has applied this approach [88, 89, 90, to hard sphere systems where the center of the hard spheres are tethered to a spherical region with a variable diameter that regulates the intensity of the coupling.

Probably up to now the most powerful method to compute numerically the configuration entropy numerically is the one based on the IS formalism. Moreover, due to its relatively simple implementation, the IS formalism has become an important tool in the numerical analysis of models. For this reason we shall give a more detailed presentation. The calculation of IS, summarized in Figure 5 follows directly from the definition. First the system is equilibrated at a given temperature $T$, then starting from an equilibrium configuration the system is instantaneously quenched down to $T=0$ by decreasing the energy along the steepest descent path. The procedure is repeated several times starting from uncorrelated equilibrium configurations. In this way the IS are identified and their probability distribution can be computed. In equilibrium at temperature $T$ the system explores the IS of energy $E_{\mathrm{IS}}=E$ with probability [see (106), 112

$$
\mathcal{P}(E, T)=\exp \left[S_{c}(E)-\beta E-\beta F_{v}(T, E)+\beta F(T)\right]
$$

where $F(T)$ is the equilibrium free energy. Then the SW configurational entropy can be computed just inverting this relation:

$$
S_{c}(E)=\ln \mathcal{P}(E, T)+\beta E+\beta F_{v}(T, E)-\beta F(T)
$$

and using the computed IS probability distribution. If the energy dependence of $F_{v}(T, E)$ can be neglected, then curves for different temperatures can be superimposed and the resulting curve is, except for an unknown constant, the SW complexity $S_{c}(E)$. The unknown constant can be fixed either comparing the numerical results with 
known theoretical predictions, or using the method described below. This method works rather well for some disordered spin systems as the Random Orthogonal Model (ROM), where the data collapse is rather good for a quite large energy interval [65]. The vibrational contribution $F_{v}$ follows from the motion inside the component. Its independence from the energy of IS means that all components are equivalent, i.e., have similar shapes. In general this is not the case and its contribution must be included. For systems with continuous variables $F_{v}$ can be calculated at low $T$ in the harmonic approximation by expanding the energy about the IS configuration [91, 92].

An alternative numerical method consists in computing directly the configurational entropy as function of temperature. This method is free from unknown constants but does not resolve the configurational entropy as function of IS energy. Considering (110) and (112) we have

$$
\begin{aligned}
F(T) & =E(T)-T S(T) \\
& =E_{\mathrm{IS}}(T)+E_{v}(T)-T S_{c}(T)-T S_{v}(T) .
\end{aligned}
$$

where $E_{\mathrm{IS}}(T)$ is the average energy of IS seen at equilibrium at temperature $T$. The total entropy is then the sum of two contributions

$$
S(T)=S_{c}(T)+S_{v}(T)
$$

The first term accounts for the multiplicity of components of energy $E_{\mathrm{IS}}(T)$ while the second for their "volume". The SW configurational entropy can then be computed as difference between the total and the vibrational entropy.

The total entropy $S$ can be evaluated via thermodynamic integration of the total energy at temperature $T$ from a known reference point:

$$
\Delta S=S(T)-S\left(T^{*}\right)=\int_{T^{*}}^{T} \frac{d E}{T} .
$$

To compute the vibrational contribution is more difficult, however at low temperature the system mainly explores the bottom of the components, near the IS. If the system is described by continuous variables then the vibrational contribution can be computed in the harmonic approximation by expanding about the IS. This leads to

$$
S_{v}(T) \simeq S_{\text {harm }}(T)=\mathcal{N}-\sum_{i=1}^{\mathcal{N}} \log \left[\frac{\hbar \omega_{i}(T)}{k_{B} T}\right]
$$

where $\omega_{i}$ is the (average) frequency of the $i$-th normal mode and $\mathcal{N}$ the number of normal modes. It is possible to refine this approximation by adding terms which take into account the basins anharmonicities, however usually these are negligible when compared with (124) 92. For systems with discrete variables, as for example disordered Ising-spin systems, the vibrational contribution can be estimated from the $T \rightarrow 0$ expansion of the Thouless Anderson Palmer entropy, which leads to

$$
S_{v}(T) \simeq \sum_{i=1}^{N} 2 \beta\left|h_{i}\right| \exp \left(-2 \beta\left|h_{i}\right|\right)
$$

where $N$ is the number of spins, and $h_{i}$ is the local field acting on $i$-th spin evaluated at the IS configuration 93 .

These methods have been successfully applied to several model systems with both continuous variables such as Lennard-Jones glasses 91, 94, 92 or discrete variables like the ROM [65] or the SK model 65, 95]. 
Recently in [96] it has been introduced a numerical method to compute directly $S_{c}(\mathcal{F}, T)$ within the IS decomposition scheme based on the probabilistic definition of the component free energy. The dynamical evolution of the system in equilibrium defines a probability measure $p_{\mathcal{R}}$ over the components. In the case of an ergodic dynamics, and assuming that the observation time $\tau_{\text {obs }}$ is larger than the equilibration time, the statistical weight of the component $\mathcal{R}$ is:

$$
p_{\mathcal{R}}(T)=\frac{\tau_{\mathcal{R}}}{\tau_{\text {obs }}}=\exp [-\beta F(\mathcal{R})+\beta F(T)]
$$

where $\tau_{\mathcal{R}}$ denote the time spent by system in the component $\mathcal{R}$ during the total observation time $\tau_{\text {obs }}, F(T)$ the equilibrium free energy and $F(\mathcal{R})$ the component free energy [See (75)]. The probability to find at temperature $T$ a component with free energy equal to $\mathcal{F}$ is

$$
\begin{aligned}
\mathcal{P}(\mathcal{F}, T) & =\sum_{\mathcal{R}} p_{\mathcal{R}}(T) \delta(\mathcal{F}-F(\mathcal{R})) \\
& =\exp \left[S_{c}(\mathcal{F}, T)-\beta \mathcal{F}+\beta F(T)\right]
\end{aligned}
$$

so that

$$
S_{c}(\mathcal{F}, T)=\ln \mathcal{P}(\mathcal{F}, T)+\beta[\mathcal{F}-F(T)] .
$$

If the number of different components is not too large $F(\mathcal{R})$ can be estimated directly using (126) and the frequency with which a given component $\mathcal{R}$ appears in a (long) simulation at temperature $T$ :

$$
F(\mathcal{R})=-T \ln \left(\frac{\tau_{\mathcal{R}}}{\tau_{\text {obs }}}\right)+F(T)
$$

The equilibrium free energy $F(T)$ can be computed by performing simulations at different temperatures and integrating the energy $E(T)$ of the system from $T=\infty$ down to $T$ :

$$
\beta F(T)=\int_{0}^{\beta} d \beta^{\prime} E_{\mathrm{eq}}\left(\beta^{\prime}\right)-S(\beta=0) .
$$

where $S(\beta=0)$ is the infinite temperature entropy of the system. From the value of $F(\mathcal{R})$ it is now easy to construct the histogram $\mathcal{P}(\mathcal{F}, T)$ and using (128) compute $S_{c}(\mathcal{F}, T)$. Because the system is equilibrated, in this approach components with identical free energy are sampled with the same probability. This differs from the previous method where components with the same energy $E_{\mathrm{IS}}$ are assumed to be equally probable which is clearly an approximation. The two methods coincide only if components have similar volumes so that the component entropy is the same.

This method has been successfully applied in 96 to the study of the ROM and the SK model, two cases with completely different critical behaviour. In both cases the computed potential $\Phi$ allows for a very precise calculation of critical temperatures using relatively small systems giving confidence on $S_{c}(\mathcal{F}, T)$. Moreover the form of $\Phi$ clearly discriminates between the two different types of transitions.

\subsection{The concept of the effective temperature}

There is the long standing idea that the non-equilibrium regime in aging or driven systems can be characterized by the FDR $X_{A, B}(C)$ (94) that has the meaning of a 
temperature in the thermodynamic sense. This suggestion stems from the observation that (94) can be recast in the following form,

$$
\frac{1}{T_{\mathrm{eff}}^{(A, B)}(C)}=\frac{X_{A, B}(C)}{T}=\left.\frac{R_{A, B}(t, s)}{\frac{\partial}{\partial s} C_{A, B}(t, s)}\right|_{C_{A, B}(t, s)=C}, \quad t>s(131)
$$

defining an effective temperature through the relation

$$
T_{\mathrm{eff}}^{(A, B)}(C)=\frac{T}{X_{A, B}(C)}
$$

As defined in (131), (132) the effective temperature is nothing else than a suitable parameter which tells that the QFDT becomes the usual FDT by replacing the bath temperature with the effective temperature. In many cases $X_{A, B}(C)<1$ so the effective temperature $T_{\text {eff }}^{(A, B)}$ is larger than the bath temperature.

The idea that some concepts of thermodynamic systems can be applied also to non-equilibrium systems has been wandering around in the literature for a long time (in the context of turbulence see [97] or in the context of structural glasses [2, 98, 99]). The statement that the effective temperature (132) has indeed a thermodynamic meaning faces some conceptual problems and difficulties not found in equilibrium theory. $T_{\mathrm{eff}}^{(A, B)}$ should satisfy the following properties,

- Observable independence. $T_{\mathrm{eff}}^{(A, B)}(C)$ must be independent of the observables $A, B$ used to construct correlations and responses. If this is not always possible, as the present numerical evidence suggests, at least one would still like to know beforehand which set of "good" observables endow (132) with a physical meaning. These observables have received the name of neutral observables and have been discussed in section 4.4 From many perspectives, this condition appears quite strong. It could be relaxed by only requiring independency of $T_{\text {eff }}$ upon the measured observable $A$ for a given perturbation $B$ (rather than on both $A, B$ ).

- Zero ${ }^{\text {th }}$ law. If the slow degrees of freedom of a system described by effective temperature $T_{\text {eff }}(C)$ (we have dropped the $A, B$ dependence) are put in contact with a thermal bath at temperature $T$, the net heat flow between the system and the bath should vanish only if $T_{\text {eff }}(C)=T$, where $C$ determines the relevant timescale (as described in section 4.1) at which the thermal bath, acting as a thermometer, responds. The same conclusion must hold between two glassy systems described by two effective temperatures $T_{\text {eff }}^{(1)}(C), T_{\text {eff }}^{(2)}(C)$. After putting them in contact the net heat current between them, at the relevant timescale defined by the correlation $C$, vanishes only if $T_{\mathrm{eff}}^{(1)}(C)=T_{\mathrm{eff}}^{(2)}(C)$. This definition, apparently reasonable, encounters some difficulties that we will describe below. In particular, systems with identical effective temperatures $T_{\text {eff }}^{(1)}(C)=T_{\text {eff }}^{(2)}\left(C^{\prime}\right)$ but at different timescales $\left(C \neq C^{\prime}\right)$ cannot be in mutual equilibrium.

- Existence of a non-equilibrium measure. The zero $^{\text {th }}$ law carries associated a maximum principle. In standard thermodynamics the zero $^{\text {th }}$ law establishes that after putting in contact two systems at different temperatures the global system reaches a stationary state with a unique temperature. This stationary state is the one that maximizes the global entropy of the compound system compatible with a given total energy. Moreover fluctuations around this maximum entropy state are ruled by the temperature. By the same token, the aging state of relaxational systems and the stationary state of driven systems must exhibit some fluctuations 
or deviations around the aging (or driven) state that are described by the effective temperature $T^{\mathrm{eff}}(C)$. The full characterization of these fluctuations is presently unknown.

In Section 4.2 we tried to fortify the idea that a thermodynamic description is indeed possible and that the effective temperature shares some properties of thermodynamic temperatures. Cugliandolo, Kurchan and Peliti [100] have emphasized these aspects showing that the effective temperature can be defined only regarding the timescale under consideration. They considered a small thermometer that can be mimicked by a single harmonic oscillator of frequency $\omega$ that is put in contact with the original system. For definitiveness let us consider that $x$ denotes the oscillator coordinate and $O(y)$ an observable of the system (described by the variable $y$ ) to which the oscillator is coupled by an interaction term, $-\epsilon x O(y)$ where $\epsilon$ is the intensity of the coupling. If $\epsilon$ is small enough, then the interaction between the oscillator and the system can be treated within the linear-response theory and the energy of the oscillator evaluated in the stationary state. The equipartition theorem relates this energy to the temperature measured by the oscillator acting as a thermometer. In aging systems, the effective temperature is given by the FDT in the frequency domain (also called Nyquist formula),

$$
T_{\mathrm{eff}}^{(O)}\left(\omega, t_{w}\right)=\frac{\pi}{2} \frac{\omega S_{O}\left(\omega, t_{w}\right)}{\chi_{O}^{\prime \prime}\left(\omega, t_{w}\right)}
$$

where $S_{O}\left(\omega, t_{w}\right)$ is the power spectrum or correlation $\left\langle O(t) O\left(t_{w}\right)\right\rangle$ expressed in Fourier space (see (275) ) and $\chi_{O}^{\prime \prime}\left(\omega, t_{w}\right)$ the corresponding out-of-phase susceptibility. A similar expression is valid for the stationary non-equilibrium state of driven systems, however because TTI holds the $t_{w}$ dependence in (133) drops off. The connection between (132) and (133) appears when translating the meaning of $\omega$ and $t_{w}$ into the many timescales scenario. According to that $\omega$ corresponds to $1 /\left(t-t_{w}\right)$ and therefore we can define $C^{*} \equiv C\left(t, t_{w}\right)=C\left(t_{w}+1 / \omega, t_{w}\right)$. This means that a thermometer put in contact with the system at time $t_{w}$ and responding at a given frequency $\omega$ measures the temperature $T_{\text {eff }}\left(C^{*}\right)=T / X\left(C^{*}\right)$. Equivalently, to measure the temperature $T_{\text {eff }}\left(C^{*}\right)=T / X\left(C^{*}\right)$ in an aging system a thermometer responding to a time scale $t^{*}=1 / \omega$ with $C^{*} \equiv C\left(t_{w}+1 / \omega, t_{w}\right)$ should be used. In aging systems with twotimescales (such as structural glasses) characterized by the full aging $t / t_{w}$, in order to measure the effective temperature associated to the slow process, the frequency of the thermometer must be $\omega \sim 1 / t_{w}$. The thermometer must respond in a timescale of the order of the waiting time!!. In this scenario, effective temperatures can be extremely difficult to measure and this raises the question about their true meaning as the system drifts away from that state in the time required for a single measurement. To cope with this problem it has been proposed [101] that an ensemble of small thermometers is needed for the measurement. However, this does not solve the problem of how to measure, using this procedure, the effective temperature of a vitrified piece of glass quenched one thousand years ago. These difficulties are inherent to aging systems but not necessarily in driven systems that reach a stationary TTI state. For these reasons, experimental measurements of FDT violations and effective temperatures could be more suitable in driven rather than aging systems (concerning experiments see discussion in section 8 .

Zero $^{\text {th }}$ law aspects of the effective temperature have been considered in 100, 102 within the spherical $p$-spin model. It has been shown that in the large $t_{w} \rightarrow \infty$ limit and low frequency limit $\omega \rightarrow 0$ there are only two possible Ansatz solutions 
that close the dynamical equations in the aging state: either the two systems remain uncoupled with different effective temperatures or they thermalize and reach a common temperature. These results have been endorsed by a systematic study of these coupled solutions in the framework of the oscillator (OSC) model [103. The OSC model is characterized by a single timescale corresponding to the slow process at zero temperature (see section 6.5.1). It has been shown that, in the presence of an interaction term in the Hamiltonian that describes a compound system formed by two ensembles of the OSC model, the dynamics behaves in two different ways: either the effective temperatures equalize or they are different as if the systems were uncoupled. Dynamics remains always uncoupled (independently of the coupling intensity) if dynamics is sequential on both systems, i.e., the updating is done sequentially over the two ensembles $1 \rightarrow 2 \rightarrow 1 \rightarrow 2 \ldots$. In this case the effective temperatures of the two systems differ, each one corresponding to that of the non-interacting OSC ensemble. If dynamics is parallel, i.e., updating is done simultaneously over the two OSC ensembles $1+2 \rightarrow 1+2 \rightarrow 1+2 \ldots$ and so on, then the effective temperatures of both models coincide even for a zero value of the coupling $\epsilon$ in the Hamiltonian. This result shows that, in general, two glassy systems interacting through a coupling term in the Hamiltonian do not necessarily reach the same effective temperature on timescales of the order of the waiting time.

In all these studies the same question remains always unanswered: why fast and slow degrees of freedom decouple into different effective temperatures (in twotimescales systems, one is the bath temperature, the other the (higher) effective temperature). A necessary condition is that the relaxation rate of the energy (or entropy production) decays to zero slow enough [104]. To better understand this question, in 105 the thermal current between the oscillator model and a thermometer was analyzed. There it was shown that the measured temperature $T_{\mathrm{m}}$, which makes the net current flow between system and the thermometer vanish, coincides with the effective temperature if $\omega t \sim 1$. However, in the limit $\omega t \ll 1$ the measured temperature is much smaller than the effective temperature, while in the other extreme $\omega t \gg 1$ the thermometer measures tolerably well the effective temperature. Discrepancies between the measured and the effective temperature have been also reported in the SK model in the presence of asymmetric interactions as an example of a driven system [106]. Another important aspect is that the zero ${ }^{\text {th }}$ law is hardly effective as transport coefficients (such as the thermal conductivity) are exceedingly small, in agreement with the uncoupling of degrees of freedom occurring in glassy systems with many timescales. From another point of view Nieuwenhuizen [107, 49, 108, has formulated a theory to describe the aging regime of glassy systems with two-timescales assuming, right from the beginning, that the effective temperature is indeed associated to a thermodynamic potential. In this formulation, the configurational entropy is an extensive thermodynamic potential conjugated to the effective temperature. The first law of thermodynamics, that expresses energy conservation reads $d E=d W+T d S_{\text {eq }}+T_{\text {eff }} d S_{c}$ where $S=S_{\text {eq }}+S_{c}$ is the total entropy that receives contributions from the equilibrium (or intrastate) entropy and the configurational entropy. The configurational entropy and the effective temperature manifest in the reported experimental failure of the second Ehrenfest relation (while the first is automatically satisfied by construction) leading to values of the Prigogine-Defay ratio larger than 1 [109]. 

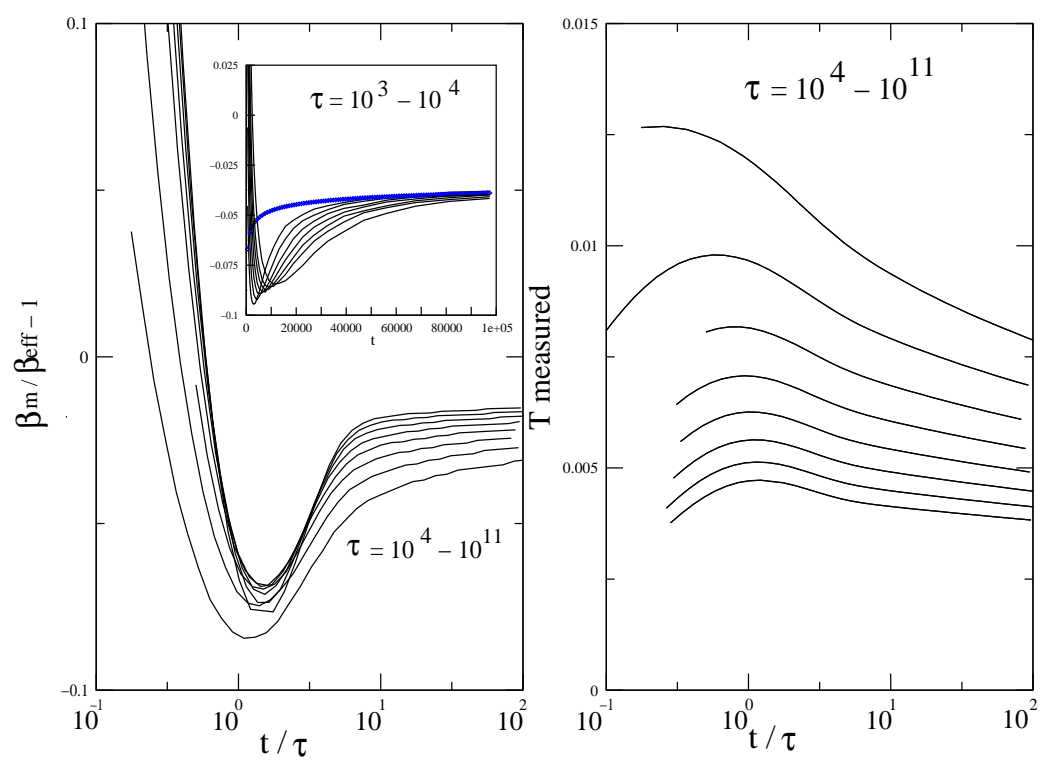

Figure 6. Left panel: Relative difference between the measured temperature by a thermometer responding in a timescale $\tau=1 / \omega$ and the glassy OSC model aged at $t$, plotted against $t / \tau$ for different values of $\tau=$ $10^{4}, 10^{5}, 10^{6}, 10^{7}, 10^{8}, 10^{9}, 10^{1} 0,10^{1} 1$ (from bottom to top). The measured temperature coincides with $T_{\text {eff }}$ for $t / \tau \sim \mathcal{O}(1)$. In the inset, the same relative difference but plotted against $t$ for $\tau=10^{3}, 10^{4}$. Right panel: Measured temperature plotted as a function of $t / \tau$. From 105.

\subsection{The Edwards' measure for granular materials}

Granular materials are systems made of a large number of individual grains such as sands or powders. At first sight granular systems look quite different from thermodynamical systems since for example they interact mainly through frictional forces and hence the energy is not conserved. Moreover power is supplied to them by tapping, shearing or shaking, all mechanisms quite different from thermal contact with a thermal bath. However, despite these differences, granular materials share with thermal systems the property that their properties are reproducible given the same set of extensive operations, i.e., operations acting upon the system as a whole and not on individual grains. For example if some sand is poured uniformly and at low density into a container one expects to have a sand of a certain reproducible density. Based on these facts it is then reasonable to hypothesize that granular systems can be described at a macroscopic level by a small number of parameter analogous, e.g., to temperature or pressure, using some ideas of statistical mechanics [110, 111, 112, 113, 114].

The most important variable describing the state of a granular system is its density, or equivalently its volume $V$. The thermal energy of a granular system at room temperature is indeed negligible. The volume $V$ is the actual volume occupied by the system, for example measured by the position of a piston in the container, and hence it depends on the configurations (position and orientation) of the grains. In principle other variables should be necessary to describe the state of the system, but if we assume that the grains are rigid then all other microscopic details can be neglected. Thus the only valid configurations of grains are stable arrangements where grains can 
remain at rest under the influence of confining forces, and with no overlap among them. We are then led to a statistical description of all possible stable configurations of grains in the real space, i.e., to a configurational statistical mechanical theory for the random packing of grains. Since in a stable configuration grains cannot move these configurations are also called blocked states.

The next step to develop a statistical mechanical description for granular systems consists in the introduction of a volume function $W$ [110, 111] which plays the role of energy in statistical mechanics. The function $W$ specifies the volume of the system in term of the configuration of grains: $V=W(\boldsymbol{q})$, where $\boldsymbol{q}$ denotes all the grain positions and orientations in a blocked state. Under the assumption that for a given volume $V$ all configurations for which $V=W(\boldsymbol{q})$ are equally probable a statistical description can be developed through a process completely analogous to that of conventional statistical mechanics. It is then possible to introduce a microcanonical ensemble with distribution function

$$
\mathrm{e}^{-S} \delta(V-W(\boldsymbol{q}))
$$

where $S=S(V)$ is the entropy defined as usual in terms of the total number of blocked states

$$
\Omega=\int d \boldsymbol{q} \delta(V-W(\boldsymbol{q})), \quad S=\ln \Omega
$$

Similar to its thermodynamic counterpart, the entropy $S$ is an extensive quantity as can be seen, for example, in simple toy models [111, 115. The measure (134)-(135) is usually called Edwards' measure for granular systems.

To define a canonical ensemble it is necessary to define a parameter analogous to temperature which characterizes the state of the system. This parameter is the compactivity $X$ defined as

$$
\frac{1}{X}=\frac{\partial S}{\partial V}
$$

and thus the partition function is

$$
\mathcal{Z}=\mathrm{e}^{-Y(X) / X}=\int d \boldsymbol{q} \mathrm{e}^{-W(\boldsymbol{q}) / X}
$$

where potential $Y(X)$ is called the effective volume and plays the role of a free-energy:

$$
Y(X)=V(X)-X S
$$

The analogy can be pushed forward and many other relations similar to that of conventional statistical mechanics can be derived. We shall not pursuit this here, however, before concluding we shall spend some more words on the compactivity $X$. The compactivity $X$ measures the packing of grains, indeed from its definition it is clear that it may be interpreted as being characteristic of the number of possible ways of arranging the grains of a system by changing the volume in an amount $\Delta V$, the change in entropy being equal to $\Delta S$. Consequently the two limits of $X$ are 0 and $\infty$ corresponding to the most compact the least compact arrangements, respectively. The compactivity $X$ also describes the balance between the tendency of the system to increase or decrease its volume and the tendency to increase or reduce its entropy.

At first sight blocked states in granular systems resemble IS discussed for glasses. Indeed a connection between the two can be drawn 116, 117, 118, 41] introducing a "tapping" dynamics for glasses, i.e., a dynamics in which each tap consists in raising the temperature and, after a short time, quenching it to zero. Similar to what has 
been done for other glassy systems one can try to describe the dynamics of the slow degrees of freedom through an effective temperature defined from the FDR 100, 119. At the mean field level this temperature turns out to coincide with the Edwards compactivity, which is related to the derivative of the entropy of blocked configurations of a given density. The Edwards ensemble immediately leads to the definition of an entropy $S_{\mathrm{Edw}}(\rho)$ as the logarithm of the number of blocked configurations of given $\rho$. The soundness of the Edwards approach encounters difficulties reminiscent of those present in the IS approach. At the present the correspondence between the Edwards' construction and the long-time slow dynamics for non mean-field models can only be checked "a posteriori" and it is presently unknown how to derive it from first principles.

\section{QFDT from exactly solvable models}

In the structural glass problem the spatial randomness is self-generated rather than put in by hand as in random spin glass models. This suggest that there should be a connection also with frustrated but regular models. In the last years several spin models, both with and without randomness, displaying structural glass transition like properties have been found. Interestingly, some of them can be solved in closed form, offering an important tool for understanding the glass transition.

In this section we shall summarize the main results on violation of FDT obtained from the exact solution of some spin models.

\subsection{The Mode-Coupling Theory}

A model Hamiltonian or an effective Lagrangian capable of describing relaxation processes in supercooled liquids and structural glasses is difficult to obtain. Early studies based on both dynamical mode-coupling theories or equilibrium densityfunctional theories suggested that there may be a close connection with mean-field spin-glass models [120]. They thus provide a set of microscopical models where glassy dynamics can be studied analytically. The basic simplification occurring in mean-field models is that after averaging over the disorder and making the number of spins very large $(N \rightarrow \infty)$ one is left with a closed set of equations for the two-times correlation and response functions. Above the a critical temperature $T_{\mathrm{c}}$ those equations admit a TTI solution satisfying the FDT. In this regime they are basically equivalent to the schematic mode-coupling equations introduced by Leutheusser, Götze and others 121, 122, 123] as a model for the ideal glass transition. Below $T_{\mathrm{c}}$ the ergodicity is broken and the FDT is violated. This is signaled by the appearance of a finite Edwards-Anderson order parameter $q_{\mathrm{EA}}$ at $T_{\mathrm{C}}$.

The fundamental quantities in the dynamical mode-coupling theory (MCT) are the local particle density correlation functions $\langle\delta \rho(\boldsymbol{x}, t) \delta \rho(\boldsymbol{x})\rangle$ where $\delta \rho(\boldsymbol{x})=\rho(\boldsymbol{x})-\rho_{0}$, with

$$
\rho(\boldsymbol{x})=\sum_{i=1}^{N} \delta\left(\boldsymbol{x}-\boldsymbol{x}_{i}\right)
$$

the local particle density, and $\rho_{0}$ the uniform fluid density

$$
\langle\rho(\boldsymbol{x})\rangle=\rho_{0} \quad \text { (homogeneous state) }
$$

where the angular brackets denote an ensemble average. In the glassy phase the system is trapped into metastable states with nonuniform (average) local density field 
$\langle\rho(\boldsymbol{x})\rangle \neq \rho_{0}$ and the density-fluctuations correlation functions do not decay to zero for $t \rightarrow \infty$ :

$$
\lim _{t \rightarrow \infty}\langle\delta \rho(\boldsymbol{x}, t) \delta \rho(\boldsymbol{x})\rangle \neq 0 .
$$

The complete mode-coupling theories lead to the time-evolution equations for the normalized correlation functions

$$
\phi_{q}(t)=\frac{\left\langle\delta \rho(\boldsymbol{q}, t)^{*} \delta \rho(\boldsymbol{q}, 0)\right\rangle}{N S_{q}}
$$

where $S_{q}=\left\langle|\delta \rho(\boldsymbol{q})|^{2}\right\rangle / N$ is the static structure factor, and $\rho(\boldsymbol{q})$ are the Fourier components of the density field $\rho(\boldsymbol{x})$

$$
\rho(\boldsymbol{q})=\int \exp (-i \boldsymbol{q} \cdot \boldsymbol{x}) \rho(\boldsymbol{x}) d \boldsymbol{x}=\sum_{i=1}^{N} \exp \left(-i \boldsymbol{q} \cdot \boldsymbol{x}_{i}\right) .
$$

The basic idea of MCT is to derive the equations of motion for the slow relaxing modes integrating out all fast modes. This leads to a set of self-consistent equations involving only slow modes variables in which all informations from fast modes are buried into density-fluctuations memory kernels of the form

$$
M_{q}(t)=i \nu_{q}+\Omega_{q}^{2} m_{q}(t)
$$

where $\nu_{q}$ is a (white-noise) frictional term arising from fast modes, $\Omega_{q}>0$ gives the frequency or time-scale of microscopic motion and $m_{q}(t)$ accounts for slow modes couplings arising from the integration of the fast modes. The general form of the MCT equations is 123 .

$\partial_{t}^{2} \phi_{q}(t)+\nu_{q} \partial_{t} \phi_{q}(t)+\Omega_{q}^{2} \phi_{q}(t)+\Omega_{q}^{2} \int_{0}^{t} d s m_{q}(t-s) \partial_{s} \phi_{q}(s)=0$

which must be solved with initial conditions:

$$
\phi_{q}(t=0)=1, \quad \partial_{t} \phi_{q}(t=0)=0 .
$$

The fundamental mechanism for the glass transition in the MCT is the feedback between slow density fluctuations expressed through $m_{q}(t)$. The solution of these equations is a formidable task since the kernel $m_{q}(t)$ involves higher-order correlations between density-fluctuation modes. Therefore when these theories are implemented approximations are generally made. The simplest approximation consists of replacing the average of products with products of averages to obtain a set of closed equations. This is some sort of mean-field approximation. Indeed within this scheme the memorykernel $m_{q}(t)$ can be expressed as a functional of the $\phi_{q}$

$$
m_{q}(t)=\mathcal{F}_{q}\left[\mathbf{V},\left\{\phi_{q}\right\}\right]
$$

with some vertex functions $\mathbf{V}$. Despite this rather strong approximation, similar to a mean-field approach, the theory contains the basic mechanism of the glass transition. We note that due to this approximation, the MCT is not capable of describing activated process, in the same way they cannot be discussed within mean-field theories. Therefore the appearance of activated-process dominated regimes is signaled in this theory by the divergence of some quantities. Activated process could in principle be included as perturbative terms, however consistent theories which account for them are not yet available. 
The main properties of the MCT can best be seen using a simplified version of the theory called schematic mode-coupling theory in which only one relaxation function is considered 121, 122, 123:

$$
\partial_{t}^{2} \phi(t)+\nu \partial_{t} \phi(t)+\Omega^{2} \phi(t)+\Omega^{2} \int_{0}^{t} d s m(t-s) \partial_{s} \phi(s)=0 .
$$

The simplest model describing an idealized structural glass transition is the one specified by the two coupling constants $\left(v_{1}, v_{2}\right)$ :

$$
m(t)=v_{1} \phi(t)+v_{2} \phi(t)^{2} .
$$

This theory predicts a transition from an ergodic liquid phase, where $\phi(t \rightarrow \infty) \rightarrow 0$, to a glass phase, where the ergodicity is broken and $\phi(t \rightarrow \infty) \rightarrow f>0$, as the parameter $\left(v_{1}, v_{2}\right)$ are varied. Depending on the values of $\left(v_{1}, v_{2}\right)$ the nature of the transition can be either continuous (type A) with $f$ growing continuously from zero or discontinuous (type B) with $f$ jumping from zero to a finite value as the transition line is crossed.

\subsection{Disordered spin-glass models}

Mean-field spin-glass models can be classified into two broad classes depending on the value of the Edwards-Anderson parameter $\left(q_{\mathrm{EA}}\right)$ at the transition (for the structural glass transition this can be identified with the long-time limit of the density correlation functions). The first class, called discontinuous models, includes models for which a finite $q_{\mathrm{EA}}$ appears discontinuously at $T_{\mathrm{c}}$. The prototype model in this class, which we discuss here, is the spherical $p$-spin model. Other models included in this family are, Potts-glasses with more than four components [124, 42, quadrupolar glass models 124,125 and $p$-spin interaction spin-glass models [126, 127, 128, 43, 44]. The second class includes models, such as the Sherrington-Kirkpatric (SK) model [129] 130, for which $q_{\mathrm{EA}}$ starts continuously from 0 at $T_{\mathrm{c}}$. Those models are termed continuous models.

6.2.1. p-spin spherical model. Among the mean-field spin-glass model with a discontinuous spin glass transition an important role has been played by the spherical $p$-spin spin-glass model. Spin-glass models with multispin interactions were first considered in the eighties for both Ising [126, 127, 128] as well as soft spins [43, 44]. However, while the static properties could be computed for arbitrary values of $p$, dynamical properties were limited to values of $p$ close to 2 [43, 44]. An important step forward came with the introduction of the spherical $p$-spin spin-glass model 131 132, since its statics and dynamics can be solved in closed form for any value of $p$.

The spherical $p$-spin interaction spin-glass model is defined by the Hamiltonian

$$
\mathcal{H}=-\sum_{1 \leq i_{1}<\cdots<i_{p} \leq N} J_{i_{1} \ldots i_{p}} \sigma_{i_{1}} \cdots \sigma_{i_{p}}-h \sum_{i=1}^{N} \sigma_{i}
$$

where $h$ is an external field, which in the following we shall take equal to zero for simplicity. It describes a system of $N$ continuous spins $\sigma_{i}$ interacting via randomly quenched infinite range $p$-spin interactions $J_{i_{1} \ldots i_{p}}$ which are taken to be Gaussian with zero mean and variance

$$
\overline{\left(J_{i_{1} \ldots i_{p}}\right)^{2}}=\frac{J^{2} p !}{2 N^{p-1}}, \quad 1 \leq i_{1}<\cdots<i_{p} \leq N .
$$


The overbar stands for the average over the couplings. The scaling with $N$ is chosen such that there is a well defined thermodynamic limit $N \rightarrow \infty$. The spins can vary continuously from $-\infty$ to $+\infty$, but are subject to the global spherical constraint

$$
\sum_{i=1}^{N} \sigma_{i}^{2}=N
$$

which must be satisfied at any time. The relaxational dynamics for $\sigma_{i}(t)$ is given by the set of Langevin equations 133

$$
\Gamma_{0}^{-1} \partial_{t} \sigma_{i}(t)=-r(t) \sigma_{i}(t)-\frac{\delta \beta \mathcal{H}}{\delta \sigma_{i}(t)}+\eta_{i}(t)
$$

The kinetic coefficient $\Gamma_{0}$ sets the time scale of the microscopic dynamics, and will be henceforth set to 1 without loss of generality, while $\beta=1 / T$. The last term in (153) $\eta_{i}(t)$ is a Gaussian random field with zero mean and variance

$$
\left\langle\eta_{i}(t) \eta_{j}\left(t^{\prime}\right)\right\rangle=2 \Gamma_{0}^{-1} \delta_{i j} \delta\left(t-t^{\prime}\right)
$$

representing the effects of thermal noise. The average over thermal noise is denoted as usual by angular brackets $\langle\cdots\rangle$. The first term in (153) enforces the spherical constraint at any time, and must be fixed self-consistently. In the mean-field limit $N \rightarrow \infty$ the sample-averaged dynamics is entirely described by the evolution of the two-times correlation and linear response functions

$$
\begin{aligned}
& C(t, s)=\overline{\left\langle\sigma_{i}(t) \sigma_{i}(s)\right\rangle} \\
& R(t, s)=\left.\frac{\delta \overline{\left\langle\sigma_{i}(t)\right\rangle}}{\delta h_{i}(s)}\right|_{h_{i}=0}
\end{aligned}
$$

which vanishes for $t<s$ due to causality. The dynamical equations for $C$ and $R$ are obtained from (153) 132] through standard functional methods [134, 43, 44]. At high temperatures the system is ergodic, thus for initial time $t_{\mathrm{i}} \rightarrow-\infty$ both TTI and FDT hold. Using the FDT relation the equation for $C(t-s)$ reads [132]

$$
\partial_{\tau} C(\tau)+C(\tau)+\int_{0}^{\tau} d \tau^{\prime} m\left(\tau-\tau^{\prime}\right) \partial_{\tau^{\prime}} C\left(\tau^{\prime}\right)=0
$$

where $\tau=t-s$ and

$$
m(\tau)=\mu C(\tau)^{p-1}
$$

which has the same structure of the schematic MCT equation (148). The correlation always decays to zero for large $t$. However slightly above $T_{\mathrm{c}}$ is develops a plateau at $C \sim q_{\mathrm{EA}}$ before eventually decaying to zero, see Figure $\mathbf{7}$ The length $\tau_{p}(T)$ of the plateau increases as a power of $T-T_{\mathrm{c}}$ and diverges at $T_{\mathrm{c}}$. Near the plateau one finds that:

$$
\begin{aligned}
& C(\tau) \sim q_{\mathrm{EA}}+c_{a} \tau^{-a}, \quad C \gtrsim q_{\mathrm{EA}} \\
& C(\tau) \sim q_{\mathrm{EA}}-c_{b} \tau^{b}, \quad C \lesssim q_{\mathrm{EA}}
\end{aligned}
$$

where the exponent $a$ and $b$ are related by:

$$
\frac{\Gamma^{2}(1-a)}{\Gamma(1-2 a)}=\frac{\Gamma^{2}(1+b)}{\Gamma(1+2 b)}=\frac{(p-2)\left(1-q_{\mathrm{EA}}\right)}{2 q_{\mathrm{EA}}}
$$

The plateau length scale $\tau_{p}(T)$ sets the equilibration time-scale. Therefore, as the temperature is lowered down to $T_{\mathrm{c}}$ the system undergoes a transition since the length of 


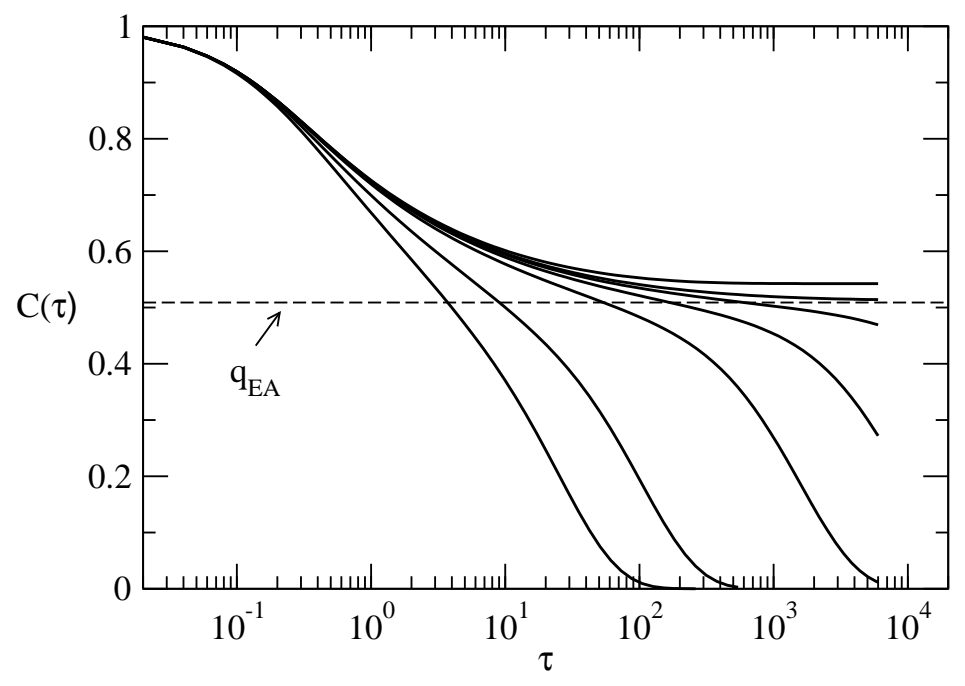

Figure 7. $C(\tau)$ as function of time for the spherical $p$-spin model with $p=3$. Left to right: $T=0.7,0.65,0.62,0.615,0.613,0.612$ and 0.61 . The horizontal line is $q_{\mathrm{EA}}\left(T=T_{\mathrm{c}}\right) \sim 0.509034$ and $T_{\mathrm{c}}=0.612372$

the plateau diverges and the correlation fails to decay to zero. In the low-temperature spin-glass phase $\left(T<T_{\mathrm{c}}\right)$ the system cannot equilibrate and the ergodicity is broken so the state of the system may depend on its initial state. In this scenario it is clear that even a mean-field theory can be highly non-trivial. To discuss the dynamics in the low-temperature phase it is convenient to have an understanding of the lowtemperature structure of the phase-space. A standard method to deal with such a problem is the so called replica trick, see e.g. 35. The breaking of ergodicity results in a breaking of the permutation symmetry between replicas 135]. The general form of the breaking is, however, not known. For $p$-spin models it has been found that the solution is given by the so called one-step replica symmetry breaking (1RSB) form [127, 128, 44, 131]. Physically this means that the phase space is broken into equivalent ergodic components separated by infinite (for $N \rightarrow \infty$ ) barriers and the equilibrium solution is described by only three parameters: the overlap between two different ergodic components, the overlap inside an ergodic component (the EdwardsAnderson order parameter) and the probability that two different replicas will be found in the same ergodic component. For the $p$-spin spherical model this solution is valid everywhere in the low-temperature phase [131. Ising-spin $p$-spin models present an even lower temperature phase with a more complex structure described by an infinite step replica symmetry breaking ( $\infty$-RSB) 128. A similar scenario is also found in Potts glasses with more than four components 124 .

Besides the replica approach, which gives essentially information on the lowestlying states which mostly contribute to the equilibrium measure, a good understanding of the landscape topology is given by the TAP approach. Using these methods we have now a rather good knowledge of the landscape 136, 73, 85, 137, 138, 139, 140. Roughly speaking the picture that emerges for the $p$-spin spherical model is that equilibrium states, whose energy and free energy difference is $O(1)$, are separated by $O(N)$ barriers. As the temperature is changed the solutions neither merge nor bifurcate, and their free energy smoothly changes. The TAP equations for the $p$-spin spherical model have 
non-trivial solutions in the free energy range $\left(F_{1 \mathrm{rsb}}, F_{\mathrm{thr}}\right)$, where the threshold free energy $F_{\text {thr }}$ is larger that the equilibrium free energy $F_{1 \text { rsb }}$ by an $O(N)$ quantity, the difference being the complexity, see section [5.1] which is maximal for $F=F_{\text {thr }}$ and vanishes when $F=F_{1 \mathrm{rsb}}$. Below the threshold the equilibrium states are local minima of the TAP free energy separated by $O(N)$ barriers, while above the threshold there are no minima.

To have a meaningful investigation of the non-ergodic phase some regularization scheme of the dynamics on a very long time-scale is required. One possibility is to refer to a large finite system. The finiteness of $N$ guarantees ergodicity by allowing the penetration of barriers whose height would diverge for $N \rightarrow \infty$ limit, and the system can equilibrate. This approach was first proposed by Sompolinsky for the Sherrington-Kirkpatrick model [141]. In the Sompolinsky's scheme TTI holds and FDT is satisfied up to some time-scale $t_{0}$, which diverges as $N \rightarrow \infty$, related to the (free)energy barrier crossing but it is violated for time-scales larger than $t_{0}$ where it is replaced by a modified form called quasi fluctuation-dissipation theorem (QFDT). See section 6.2 .2 for a more detailed discussion of the Sompolinsky's scheme. In order to consider the motion on the two different time regime $\left(t \ll t_{0}\right.$ and $\left.t \gg t_{0}\right)$ one writes 32, 132 .

$$
C(\tau)=C_{1}(\tau)+C_{0}(\xi), \quad R(\tau)=R_{1}(\tau)+\frac{1}{t_{0}} R_{0}(\xi)
$$

where $\xi=\tau / t_{0}$. The functions $C_{1}(\tau)$ and $R_{1}(\tau)$ describe the motion in a single ergodic component and vary on time-scales $\ll t_{0}$, while the functions $C_{0}(\xi)$ and $R_{0}(\xi)$ describe the motion among different minima and hence vary on time-scales $\gg t_{0}$. Continuity imposes

$$
\begin{array}{ll}
C_{1}(\tau=0)=1-q_{\mathrm{EA}}, & C_{1}(\tau \rightarrow \infty)=0 \\
C_{0}(\xi=0)=q_{\mathrm{EA}}, & C_{0}(\xi \rightarrow \infty)=0
\end{array}
$$

thus $C_{0}$ describes the slow decay of correlation function from $q_{\mathrm{EA}}$ to zero. The initial conditions for $R_{0}, R_{1}$ are obtained from the FDT, the QFDT and the continuity condition for $C$.

On time-scales $\tau \ll t_{0}$ FDT holds, so $C_{1}(\tau)$ obeys a dynamical equations similar to (157). The equations for $C_{0}(\xi)$ and $R_{0}(\xi)$ are more complex, however it can be proved [132] that they are related by the QFDT relation

$$
R_{0}(\xi)=-\beta x \theta(\xi) \partial_{\xi} C_{0}(\xi)
$$

with $0 \leq x \leq 1$ given by:

$$
x=\frac{(p-2)\left(1-q_{\mathrm{EA}}\right)}{q_{\mathrm{EA}}}
$$

Within the replica formalism the parameter $x$ corresponds to the location of the discontinuity in the order parameter $q(x)$. However at difference with the static calculation, where $x$ is fixed by the requirement of stationarity of the replica free energy $F$ with respect to $x$, the dynamical calculation requires $\partial_{x} F$ be maximal (marginal condition) 132. This condition is equivalent to the condition of a maximal configurational entropy [42, 73, 77], so that the dynamics is dominated by the states with the largest degeneracy (threshold states).

The Sompolinsky approach has several similarities with the static approach, and indeed in the static limit it correctly reproduces the static results obtained within the Parisi scheme. However it suffers of some problems which are difficult to amend since 
it would require the detailed knowledge of the dynamics for a large but finite system. One among the most serious inconsistencies of the Sompolinsky dynamics is that FDT violations, as given in (165), satisfy TTI. This is untenable in the aging regime of purely relaxational systems (although not necessarily in driven systems) where TTI is clearly violated. It must be noted though, that the Sompolinsky approach was never proposed to explain aging, since it is eliminated from the theory at the beginning.

For this reason there have been various attempts to amend the solution. One possibility, proposed by Horner [32, 142, 143, 132, consists in cooling the system, at finite cooling rate the system from $T>T_{\mathrm{c}}$ to $T<T_{\mathrm{c}}$. This introduces a regularization time scale, the inverse of cooling rate, which is eventually sent to infinity at the end. Another approach [32, consist in making the disorder time-dependent hence restoring ergodicity on time-scales larger than the disorder typical time-scale.

The above methods assume in one way or another equilibrium, thus cannot describe non-equilibrium properties typical of glasses such as aging, see section 4.1 To tackle them a different scheme has been proposed by Cugliandolo and Kurchan [52]. The main difference lies in that the thermodynamic limit is taken before any large time limit, including the initial limit $t_{\mathrm{i}} \rightarrow-\infty$. At difference with the Sompolinsky's approach this is a non-equilibrium scheme since the system evolves from a nonequilibrated initial configuration. As the system evolves in time the dynamical freeenergy density decreases, and the systems explores an ever decreasing portion of phase space. The weak ergodicity breaking [40] describes this non-equilibrium regime before equilibrium is reached. In this scenario the important parameter is the waiting time $t_{w}$, i.e., the time elapsed since the quench into the low-temperature phase. For longer waiting times the system can explore deeper minima becoming less susceptible to external perturbations an hence ages. The weak ergodicity breaking scenario can be summarized in the following assumptions.

(i) After any time $t_{w}$ the system continues to drift away and asymptotically reaches the maximum allowable distance. Thus the correlation functions satisfy

$$
\partial_{\tau} C\left(\tau+t_{w}, t_{w}\right) \leq 0, \quad \partial_{s} C(t, s) \geq 0, \quad(t>s)
$$

and in the absence of external magnetic fields

$$
\lim _{\tau \rightarrow \infty} C\left(\tau+t_{w}, t_{w}\right)=0 \quad \text { for any fixed } t_{w}
$$

(ii) The response to a constant small magnetic field applied from $s=0$ to $s=t_{w}-$ i.e., the TRM $M^{\mathrm{TRM}}\left(t, t_{w}\right)$ - decays to zero after long enough times

$$
\lim _{t \rightarrow \infty} \int_{0}^{t_{w}} d t^{\prime} R\left(t, t^{\prime}\right)=\lim _{t \rightarrow \infty} M^{\mathrm{TRM}}\left(t, t_{w}\right)=0
$$

for any fixed $t_{w}$.

(iii) The evolution of the two-times correlation function presents two distinct regimes. After a long time $s$, but $\tau=t-s$ small, the correlation decay from 1 at equal time to a plateau value $q_{\mathrm{EA}}$ defined as:

$$
q_{\mathrm{EA}}=\lim _{\tau \rightarrow \infty} \lim _{s \rightarrow \infty} C(\tau+s, s) .
$$

This fast decay corresponds to a fast relaxation toward a local minimum. In this time-sector the system behaves as if it were in a local equilibrium, and both TTI and FDT hold. The value of $q_{\mathrm{EA}}$ measures the size of the local minima or, equivalently, the width of the channel through which the system evolves. This 
fast relaxation is followed by a slow decay of $C$ below $q_{\mathrm{EA}}$ and the exploration of different minima. Since the depth of minima increases with time, $C$ decays from the plateau in a manner that depends on both $s$ and $\tau$. To show the two processes (i.e. the contribution from fast and slow motion) the response and correlation functions are split in two different terms, in a fashion similar to that used in the Sompolinsky scheme:

$$
C(t, s)=C_{\mathrm{st}}(t-s)+C_{\mathrm{ag}}(t, s), \quad R(t, s)=R_{\mathrm{st}}(t-s)+R_{\mathrm{ag}}(t, s)
$$

with

$$
\begin{array}{lc}
C_{\mathrm{st}}(t-s=0)=1-q_{\mathrm{EA}}, & C_{\mathrm{st}}(t-s \rightarrow \infty)=0 \\
C_{\mathrm{ag}}(t, t)=q_{\mathrm{EA}}, & \lim _{t \rightarrow \infty} C_{\mathrm{ag}}(t, s)=0 .
\end{array}
$$

The assumption of local equilibrium implies that FDT is satisfied by the fast motion:

$$
R_{\mathrm{st}}(t-s)=\beta \theta(t-s) \partial_{s} C_{\mathrm{st}}(t-s) .
$$

On long time-scales, however, FDT is violated and replaced by [cfr. eq (165)]

$$
R_{\mathrm{ag}}(t, s)=\beta X\left[C_{\mathrm{ag}}(t, s)\right] \theta(t-s) \partial_{s} C_{\mathrm{ag}}(t, s)
$$

with the Ansatz that $X$ depends on times only through $C_{\mathrm{ag}}$. The two forms of FDT can conveniently condensed into one extending the definition of $X$ as $X(z)=1$ for $q_{\mathrm{EA}} \leq z \leq 1$. Then we can write

$$
R(t, s)=\beta X[C(t, s)] \theta(t-s) \partial_{s} C(t, s)
$$

where $C$ and $R$ are the full correlation and response functions. For the FDT part the MCT-like equations (157) are recovered. To derive the equations for the non-FDT part the time derivatives are neglected since $C_{\mathrm{ag}}$ and $R_{\mathrm{ag}}$ are slow varying functions:

$$
\partial_{t} C_{\mathrm{ag}}(t, s) \sim \partial_{s} C_{\mathrm{ag}}(t, s) \sim 0 \quad \text { for large } t, s .
$$

As a consequence the solutions are time-reparametrization invariant, i.e., the solutions are invariant under the transformation

$$
C_{\mathrm{ag}}(t, s) \Rightarrow C_{\mathrm{ag}}(h(t), h(s)), \quad R_{\mathrm{ag}}(t, s) \Rightarrow\left[\frac{d h(s)}{d s}\right] R_{\mathrm{ag}}(h(t), h(s))
$$

where $h(t)$ is an arbitrary (well-behaved) function. The full dynamical solution obviously does not have such an invariance. This ambiguity stems from the fact that equations are only the first order equations of an asymptotic expansion. If higher order terms are included the ambiguity is removed, however we shall not discuss this problem. Motivated by the fact that the relevant time scale in slow relaxation motion is given by the waiting time, one looks for a solution of the form [52]:

$$
C_{\text {ag }}(t, s) \rightarrow C_{\text {ag }}(h(t) / h(s)) .
$$

The selection of the correct function $h(t)$ is still an open problem that requires the matching of the short and long-time regimes. Numerical solution of the dynamical equations [52] suggest a power law $h(t) \sim t^{\lambda}$.

An analysis of the correlation function near the plateau $q_{\mathrm{EA}}$ [144], similar to that done in the high-temperature phase [132], reveals the following scenario. As found in the high-temperature phase, the decay of the correlation function to the plateau $q_{\mathrm{EA}}$ is a power law with a temperature dependent exponent. The subsequent departure 
from the plateau is still a power law with another temperature dependent exponent, but at difference with the high-temperature phase it is also $t_{w}$ dependent:

$$
\begin{aligned}
C\left(\tau+t_{w}, t_{w}\right) \sim q_{\mathrm{EA}}+c_{a} \tau^{-a}, & & C \gtrsim q_{\mathrm{EA}} \\
C\left(\tau+t_{w}, t_{w}\right) \sim q_{\mathrm{EA}}-c_{b}\left(\frac{\tau}{\mathcal{T}_{w}}\right)^{b}, & & C \lesssim q_{\mathrm{EA}}
\end{aligned}
$$

with $\mathcal{T}_{w}=\left[d \ln h\left(t_{w}\right) / d t_{w}\right]^{-1}$ an effective waiting time. The exponent $a$ and $b$ are related:

$$
\frac{\Gamma^{2}(1-a)}{\Gamma(1-2 a)}=X \frac{\Gamma^{2}(1+b)}{\Gamma(1+2 b)}=\frac{(p-2)\left(1-q_{\mathrm{EA}}\right)}{2 q_{\mathrm{EA}}}
$$

where $q_{\mathrm{EA}}$ is determined by the marginal condition

$$
\begin{aligned}
& \mu(p-1) q_{\mathrm{EA}}^{p-2}=\frac{1}{\left(1-q_{\mathrm{EA}}\right)^{2}} \\
& X(C)=\frac{(p-2)\left(1-q_{\mathrm{EA}}\right)}{q_{\mathrm{EA}}} \quad \text { if } C<q_{\mathrm{EA}}
\end{aligned}
$$

and $X(C)=1$ otherwise

Here we did not consider the case of non-zero external field, this was studied in [131 132]. Another extension of the $p$-spin spherical model is the case of multiple phases treated in 145, 146, 147, 148. It is interesting to note that depending on the degree of non-linearity of the interaction three different scenarios for the transition can be observed [147. Finally, it is worth to note that the $p=2$ version of the model has the property of being solvable even for intermediate time-scales 149, 150, 151, 152, 153. or finite sizes 154.

6.2.2. The Sherrington-Kirkpatrick model. The Sherrington-Kirkpatrick (SK) model 129 130 belongs to the class of continuous spin-glass models characterized by a lowtemperature spin-glass (SG) phase of $\infty$-RSB type with a continuous order parameter function $q(x)$. The transition to the SG phase is continuous with a $q(x)$ which grows continuously from zero as $T$ is lowered below $T_{\mathrm{c}}$. Other models in this class are, e.g., the case of a particle in a long-range correlated random potential [155] or spherical models with mixture of $p=2$ and $p>3$ interactions 145] 147. For $T>T_{\mathrm{c}}$ and large times the correlation function decays to zero in the absence of external fields. However, at difference with the discontinuous SG models, it does not exhibit a plateau at $q_{\mathrm{EA}}$ for $T$ slight above $T_{\mathrm{c}}$. The SK model without external fields is defined by the Hamiltonian

$$
\mathcal{H}=-\sum_{i<j} J_{i j} \sigma_{i} \sigma_{j}
$$

where the interaction $J_{i j}$ are independent quenched Gaussian variables of zero mean and variance

$$
\overline{\left(J_{i j}\right)^{2}}=\frac{J^{2}}{N}
$$

As done previously $J$ can be set equal to 1 by rescaling the temperature. The spin variables can be either Ising spins $(\sigma= \pm 1)$ or soft spins in which case and extra term may be added to the Hamiltonian to control fluctuations. The first choice is used in static calculations, while the second is preferred in a dynamical approach. 
The $\infty$-RSB structure of the SG phase reflects a complete different topology of the phase space. Again below $T_{\mathrm{c}}$ the phase space is broken into a large number (exponentially in $N$ ) states, but now the overlap between states can take any value from 0 to $q_{\mathrm{EA}}$. The equilibrium states are organized in an ultrametric fashion with non-extensive barriers - $O\left(N^{\alpha}\right)$ with $\alpha \sim 1 / 3$ - between them. The TAP analysis shows that the TAP solutions tends to split as the temperature is lowered is a fashion similar to a second-order transition. Moreover the spectrum of the Hessian matrix of the solution extends down to zero, leaving the possibility of finite free-energy barriers. All these facts lead to a dynamical scenario quite different from that discussed above in section 6.2.1

The relaxational dynamics of the soft-spin version is given by Langevin equations similar to (153)-(154) and the self-consistent dynamical equations for the two-times correlation and response functions were first derived by Sompolinsky and Zippelius [156] 134. These are more involved than those of the spherical case because spin variables cannot be integrated away and hence will not be reported here. However, near the critical point the dynamical equations can be written in the MCT form (157), (147) with suitable $v_{1}$ and $v_{2}$ [157. The two parameters $v_{1}$ and $v_{2}$ are not independent and their particular form leaves only the possibility for the type A transition with $q_{\mathrm{EA}}$ growing continuously from zero at $T_{\mathrm{c}}$.

Above the critical temperature $T_{\mathrm{c}}$ no ergodicity breaking occurs, and the solution are TTI and satisfy FDT. Below $T_{\mathrm{c}}$ the ergodicity is broken and some scheme must be adopted for the long-time dynamics. In the Sompolinsky approach [141], as discussed in the preceding section, one assumes that the initial time is sent to $-\infty$ keeping the system size large but finite so that the system can equilibrate, and only then $N$ is sent to $\infty$. Two-time quantities as correlations and response are then trivially TTI, but FDT may not be necessarily satisfied for the infinite size system due to the emergence of infinitely high (free)energy barriers for $N \rightarrow \infty$ where freezing of some degrees of freedom confine the system to a portion of the available phase space. In the finite system, on the contrary, all barriers can be surmounted in a finite (but large) time, so the degrees of freedom can be frozen only for times smaller than the typical time-scale for barrier crossing. The large number and complex structure of states in the phase space led Sompolinsky to postulate a set of very long time-scales, eventually diverging for $N \rightarrow \infty$, for (free)energy barrier crossing. The times are organized hierarchically, i.e., denoting them with $t_{x}$, where $x$ is an index varying for convenience in $[0,1]$, then

$$
\lim _{N \rightarrow \infty} t_{x}=\infty \text {, but } \quad \frac{t_{x^{\prime}}}{t_{x}} \rightarrow \infty \quad \text { if } x>x^{\prime} .
$$

to account for the ultrametric organization of states. When the system is observed at time $t_{x} \ll t \ll t_{x^{\prime}}$ all degrees of freedom with relaxation times $t_{x}$ shorter then $t$ will have relaxed completely, while those with longer relaxation times will remain essentially frozen and cannot contribute to the response at time $t$ to an external perturbation at time zero. The FDT must then be modified to account for the missing contribution of the frozen degrees of freedom. This leads to an anomalous response term in the response function which measures the degree of FDT violation. Since barriers with time-scale larger than $t=t_{x}$ cannot be crossed the correlation function cannot decay to zero but relaxes to

$$
q\left(t_{x}\right)=\overline{\left\langle\sigma_{i}\left(t_{x}\right) \sigma_{i}(0)\right\rangle}
$$

In the thermodynamic limit these partially relaxed states will become stable states of typical size $q(x)=q\left(t_{x}\right)$ since barriers with time-scale larger than $t_{x}$ cannot 
be surmounted anymore, while those with time-scale smaller than $t_{x}$ have been already crossed several times. The overlap $q(x)$ is a non-decreasing function of $x$ and corresponds in the thermodynamic limit to the Parisi's order parameter function of the static calculation. The correlation time-persistent part $q\left(t_{x}\right)$ can be written as a sum of contributions from unrelaxed degrees of freedom [141] [cfr. (162) for one time-scale]:

$$
q\left(t_{x}\right)=\sum_{x^{\prime}<x} q_{x^{\prime}}^{\prime}
$$

Since the system is equilibrated for time-scales smaller that $t_{x}$ FDT must be satisfied on that time-scales. The presence of a time-persistent part in the correlation function and the requirement of FDT for time-scales smaller that $t_{x}$ leads to an extra term [cfr. (162) for one time-scale], called anomalous response term and denoted by $\Delta_{x}^{\prime}$, in the response function [141, 158].

Like Parisi's, Sompolinsky's derivation of the self-consistent equations for the overlap and the anomalous response term is heuristic but allows for a dynamical theory which presents many similarities with the static solution. At difference with the usual Parisi's solution, however, the Sompolinsky's solution is expressed in terms of two order parameter functions: the overlap $q(x)$ and the integrated response function $\Delta(x)$, sum of the anomalous response function terms. This extra freedom, called "gauge invariance", reflects the time-reparametrization invariance of the Sompolinsky's solution: any reparametrization $t_{x} \rightarrow h\left(t_{x}\right)$ where $h(t)$ is an arbitrary well behaved function preserving the relations (187) will lead to an acceptable solution. We have already encountered this properties when discussing non-equilibrium solutions of the spherical $p$-spin model in section 6.2.1 This fact should not be surprising since it just reflects our missing of knowledge on how the barrier-crossing time-scales diverge in the thermodynamic limit. Viceversa we may also say that this invariance is intrinsic in any mean-field solution since the details on how barriers diverge are irrelevant, the only important point is that they diverge. We note indeed that the gauge-invariance of the Sompolinsky's solution has its static counterpart in the invariance of the replica solution under replica permutations. The Parisi's solution corresponds to the "special" gauge 141]

$$
\Delta^{\prime}(x)=-\beta x q^{\prime}(x)
$$

where the prime means derivation, which relates the anomalous response term to the derivative of the overlap (or time-persistent correlation) at time-scale $t_{x}$ [cfr. (1750)]. Equation (190) known with the name of Parisi's gauge is actually an FDT relation.

As already noted in section 6.2.1 the Sompolinsky's solution, and its variants, are equilibrium solutions and cannot account for aging phenomena found in spin-glass experiments. To deal with them Cugliandolo and Kurchan have proposed a nonequilibrium scheme in which the thermodynamic limit $N \rightarrow \infty$ is taken before any large-time limit, including the initial time limit $t_{\mathrm{i}} \rightarrow-\infty$, to force the system to a nonequilibrium state [52]. This procedure drives the system to a non-equilibrium regime named weak ergodicity breaking in which TTI is lost and the system displays aging, see section 6.2.1 for more details. The TTI is recovered only for small time separations (the so called FDT regime) where the dynamics is described by Sompolinsky-like equations and the correlation function approaches the plateau $C=q_{\mathrm{EA}}$ with the power law form (180) with a temperature dependent exponent $a$.

The departure from the plateau, i.e., the aging or non-FDT regime, is more complex since the presence of many different time-scales related to different 
(free)energy barriers must be taken into account, and representations like (179) or (181) cannot be adequate. Adapting the Sompolinsky's picture of many time-scales to the weak ergodicity breaking regime the non-equilibrium relaxation from time $s$ to time $t$ is due to the crossing of (free)energy barriers with time-scales between $s$ and $t$. Then, using the time-reparametrization invariance of mean-field solutions, (179) is replaced by the asymptotic form valid for large times [53] [cfr. (189)]:

$$
C_{\mathrm{ag}}(t, s) \sim \sum_{i} C_{\mathrm{ag}}^{(i)}\left(h_{i}(t) / h_{i}(s)\right)
$$

where each contributing term $C_{\mathrm{ag}}^{(i)}$ will vary in each separate time sector defined by two successive barrier crossing and, as in the case of one time-scale, the functions $h_{i}(t)$ could be power law with a time sector dependent exponent $h_{i}(t) \sim t^{\lambda_{i}}$. Using the fact that correlations decrease as times become more and more separated, it is possible to show that for large times the following relation must hold

$$
C\left(t_{3}, t_{1}\right)=f\left[C\left(t_{3}, t_{2}\right), C\left(t_{2}, t_{1}\right)\right], \quad t_{1} \leq t_{2} \leq t_{3}
$$

where $f$ is an associative function which defines the geometry of the triangles described by the trajectory in the phase space [53]. Next one defines the fixed point $q_{i}$ of $f$ as $f\left(q_{i}, q_{i}\right)=q_{i}$. The intermediate value of the correlation between two successive fixed points defines a time-sector. We note that triangles whose side belongs to different time-scales, e.g., $C\left(t_{3}, t_{2}\right)<q_{i}$ but $C\left(t_{2}, t_{1}\right)>q_{i}$, are isosceles with $C\left(t_{3}, t_{1}\right)=\min \left[C\left(t_{3}, t_{2}\right), C\left(t_{2}, t_{1}\right)\right]$. This defines an ultrametric geometry analogous to what is found in equilibrium calculations:

$$
C\left(t_{3}, t_{1}\right)=\min \left[C\left(t_{3}, t_{2}\right), C\left(t_{2}, t_{1}\right)\right],
$$

if at least one $C\left(t_{3}, t_{2}\right), C\left(t_{2}, t_{1}\right)$ is less then $q_{\mathrm{EA}}$.

The set of fixed points $q_{i}$ can be either discrete or continuous. In the latter case the correlation (191) is the limit case of a continuous sum of infinitely many scaling functions $C_{\mathrm{ag}}^{i}$. As for the Sompolinsky's approach, the term (191) in the correlation function implies an analogous term in the response function [see (171)] which can be related to the correlation function through (175) with an $X(C)$ no more constant for $C<q_{\mathrm{EA}}$ but which coincides with the function $x(q)$ of the static treatment [159].

We note that for the spherical $p$-spin model $x(q)$ evaluated from statics is different from that evaluated from dynamics [131, 132, 52. It can be shown that a sufficient condition for the equality between the static $x(q)$ and $X(q)$ is that the system is stochastically stable [160, 161, i.e., the overlap probability distribution $P_{\epsilon}(q)$ of the system in the presence of a small perturbation must smoothly converge towards the probability distribution of the unperturbed system when $\epsilon \rightarrow 0$. Moreover the limit $\epsilon \rightarrow 0$ must also commute with the limit of large times in the dynamics. If this is the case, $x(q)$ and $X(q)$ are then equal. This result holds for mean-field spin glasses with continuous RSB such as the SK model, but not for the spherical $p$-spin model where the dynamics is dominated by long lived metastable states.

\subsection{Random manifolds and diffusive models}

The basic ingredient of a glass behavior is the appearance of a multitude of long lived states, that prevents exploration of the whole phase space. This situation is not restricted to glasses but may be present in several, apparently unrelated, far from equilibrium problems. Typical examples have been discussed in the previous sections, here we shall comment a bit on random manifolds and diffusion models. 
A typical situation where glassy behavior shows up is when studying the dynamics of an elastic manifold, with or without internal structure, in a random quenched environment. This problem appears, for example, in flux lattices in high$T_{\mathrm{c}}$ superconductors 162, interfaces in random fields 163, charge density waves, and surface growth on disordered substrates [164, 165. The competition between elastic stress and disorder produces a state with many characteristics of a glass: slow dynamics, nonlinear macroscopic response, aging and so on [166, 144]. In the meanfield limit a manifold in a random media is described by a field theory with a large number of components. In this case it is possible to derive a closed set of dynamical equations of the type discussed for spin-glass models. The model of a manifold of dimension $d$ embedded into a random medium of dimension $N$ is described by the Hamiltonian

$$
\mathcal{H}[\phi(\boldsymbol{x})]=\int d^{d} x\left(\frac{c}{2}|\nabla \boldsymbol{\phi}(\boldsymbol{x})|^{2}+V[\boldsymbol{\phi}(\boldsymbol{x}), \boldsymbol{x}]+\frac{\mu}{2} \boldsymbol{\phi}(\boldsymbol{x})^{2}\right)
$$

where the $N$ component field $\phi=\left(\phi_{1}, \phi_{2}, \ldots, \phi_{N}\right)$ gives the displacement of the manifold. The mass term $\mu$ constrains the fluctuations of the manifold to a restricted volume of the embedding space. The potential term $V$ is a Gaussian variable of zero mean and correlations

$$
\overline{V[\boldsymbol{\phi}, \boldsymbol{x}] V\left[\boldsymbol{\phi}^{\prime}, \boldsymbol{x}^{\prime}\right]}=-N \delta\left(\boldsymbol{x}-\boldsymbol{x}^{\prime}\right) \mathcal{V}\left[\frac{\left(\boldsymbol{\phi}-\boldsymbol{\phi}^{\prime}\right)^{2}}{N}\right]
$$

A common choice for $\mathcal{V}$ is

$$
\mathcal{V}(z)=\frac{(\theta+z)^{1-\gamma}}{2(1-\gamma)}
$$

where $\theta$ is the free-energy fluctuation exponent. The models are divided into "longrange" models if $\gamma<1$ and "short range" models for $\gamma>1$ since in the first case correlations grow with distance, while in the second case decay.

The study of the static (equilibrium) properties of the $d=0$ limit [167, i.e. the case of a particle moving in a random potential, reveals that the short-range case is solved by a 1RSB Ansatz, while in the opposite case of long range the full RSB scheme is needed.

The manifold dynamics is given by the usual Langevin equations:

$$
\frac{\partial}{\partial t} \boldsymbol{\phi}(\boldsymbol{x}, t)=-\frac{\delta \mathcal{H}[\phi(\boldsymbol{x})]}{\delta \phi(\boldsymbol{x}, t)}+\boldsymbol{\eta}(\boldsymbol{x}, t)
$$

where $\boldsymbol{\eta}$ is a Gaussian random process of zero mean and variance

$$
\left\langle\eta_{\mu}(\boldsymbol{x}, t) \eta_{\nu}\left(\boldsymbol{x}^{\prime}, t^{\prime}\right)\right\rangle=2 T \delta_{\mu \nu} \delta\left(\boldsymbol{x}-\boldsymbol{x}^{\prime}\right) \delta\left(t-t^{\prime}\right) .
$$

To study the long-time dynamics one introduces two-time quantities, which for the simple case of $d=0$ are the usual correlation and response functions

$$
C(t, s)=\frac{1}{N} \overline{\langle\phi(t) \cdot \phi(s)\rangle}, \quad R(t, s)=\frac{1}{N} \frac{\overline{\delta\langle\phi(t)\rangle}}{\delta \boldsymbol{h}(s)}
$$

where $\boldsymbol{h}(s)$ is a small perturbation applied at time $s<t$. In addition one also considers the mean-squared displacement correlation function

$$
\begin{aligned}
B(t, s) & =\frac{1}{N} \overline{\left\langle[\phi(t)-\phi(s)]^{2}\right\rangle} \\
& =C(t, t)+C(s, s)-2 C(t, s)
\end{aligned}
$$




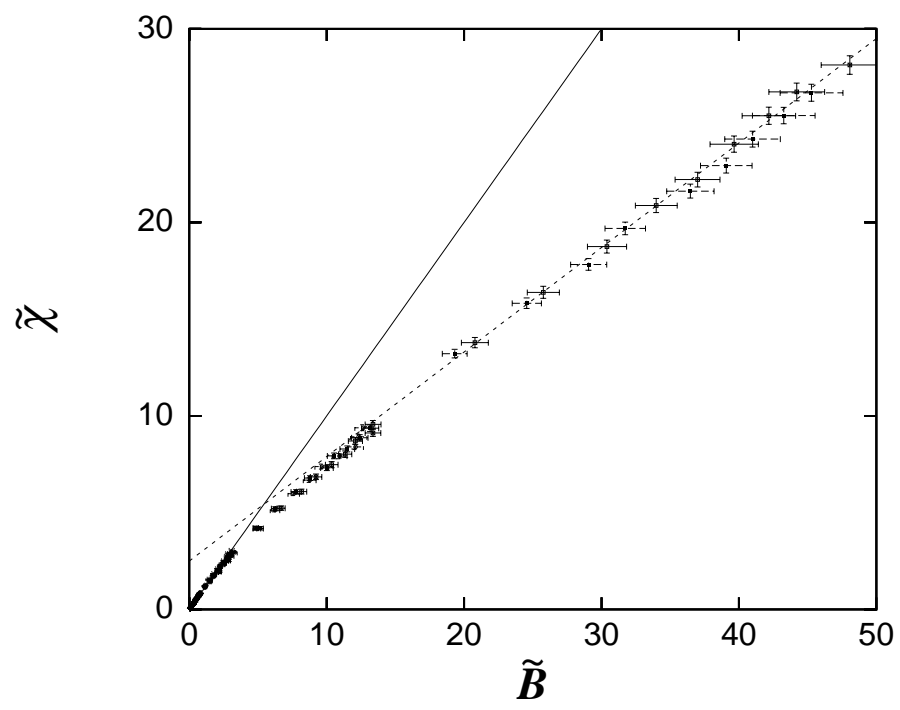

Figure 8. FD plot in the model of directed polymer in random media revealing the 1RSB or two-timescales character of the dynamics. From [168.

The analysis of the long-time dynamics for $N \rightarrow \infty$ reveals that in both cases (long and short range models), two regimes can be defined in the relaxation from an initial random configuration: (i) FDT regime for large waiting time $t_{w}$ and not too large time difference; (ii) non-FDT regime for large $t_{w}$ and time differences. Under the assumption of a weak ergodicity breaking scenario, the FDT in the non-FDT regime can be replaced by the generalized form (176) with a function $X$ which is different for short and long-range models [144]. For short range models, $X(C)$ is solved with the two-timescales Ansatz, i.e., it is 1 in the stationary sector while it is a constant smaller than 1 in the aging sector. For long-range models many time-scales are needed, and $X(C)$ is a non-trivial non-constant function, as found for the SK model. This scenario has been extended to the $d>0$ case in reference [166.

Related studies have analyzed FDT violations in polymer models. Yoshino 168 considered the directed polymer model in random media (i.e. the random manifold (194) with $c=0$ and $N=d=1$ ) and through numerical simulations confirmed the two-timescales character of aging dynamics, see figure 8 Pittard and Shakhnovich [169] have considered a heteropolymer model with uncorrelated monomer-monomer interactions. By analyzing the mode-coupling equations they found a two-timescales solution that violates FDT as reported for the random manifold model in the shortrange case.

The dynamics of the directed polymer reduces to pure diffusion in the absence of disorder. This is the well known random walk which in the continuum limit is represented by a stochastic variable $x(t)$ satisfying the (stochastic) differential equation

$$
\frac{d}{d t} x(t)=\eta(t)
$$

where $\eta$ is a Gaussian random process of zero mean and correlation

$$
\langle\eta(t) \eta(s)\rangle=2 T \delta(t-s)
$$


and $T$ is the bath temperature. A simple calculation gives for the two-time correlation and response functions

$$
\begin{aligned}
& C(t, s)=\langle x(t) x(s)\rangle=2 T \min (t, s) \\
& R(t, s)=\frac{\delta\langle x(t)\rangle}{\delta h(s)}=\theta(t-s)
\end{aligned}
$$

where $h(t)$ is an external field added to the l.h.s of eq. (201). As a consequence we see that for any $t$ and $s$

$$
X(t, s) \equiv T R(t, s) /[\partial C(t, s) / \partial s]=1 / 2
$$

a constant value different from 1 so that the FDT is violated for all times. Despite violation of FDT, the model is simpler than those discussed above, indeed the correlation and the IRF show a rather simple form as a function of the waiting time:

$$
C(t, s)=2 T s, \quad \chi(t, s)=\int_{0}^{s} d t^{\prime} R\left(t, t^{\prime}\right)=s .
$$

They both depend on $s$ but not on $t$ (as required by causality). This is a rather extreme example, however other less trivial cases, such as spinodal decomposition, scalar fields at criticality and so on also do exhibit non trivial non-equilibrium behavior [170]. More complicated non-linear diffusion effective models have been shown 171, 172, to display FDT violations compatible with a one-timescale aging scenario with a single valued FDR $X\left(t_{w}\right)<1$ that monotonically converges to 1 like in entropic models (Section 6.5).

\subsection{Trap models}

A successful family of models to describe the glass transition are phenomenological trap models. The dynamics in the aging regime can be understood in terms of jumps among different phase space components, each jump corresponding to a new rearrangement of a cooperative spatially localized region. The dynamics of the system can then be viewed as an intermittent motion where some regions remain inactive for a long time (and no net heat current is present between the system and its surroundings) until an activated jump occurs and thermal heat is released from the system to the surroundings, and from there, to the thermal bath. Phenomenological trap models, contrarily to mode-coupling theories, are based on the activated nature of glassy dynamics. Although trap models have appeared from time to time in the literature (see [39] and references therein) the most recent and successful study is due to Bouchaud [40] who has considered its relevance to describe aging phenomena in glassy systems.

The trap model corresponds to a set of unstructured energy (or free energy) traps that live in a "free energy space" without any explicit reference to real-space configurations. It corresponds, in many aspects, to the coarse-grained description developed in section 4.2 where activated processes are represented as transitions between different phase space components $\mathcal{R}$ that here could be visualized as traps. The number of traps, like the number of components $\mathcal{R}$ in the coarse grained description of the phase space, is exponentially large with the volume of the system. The model is defined by a set of traps of different depths $E$ (with $E>0$ ) with a density $\rho(E)$ and a distribution of escape times given by the Arrhenius expression $\tau(E)=\tau_{0} \exp (E / T)$ where $\tau_{0}$ is a microscopic time and $T$ is the temperature of the bath. Note that, in this last expression, the top-level for all barriers is fixed at zeroheight. The dynamics of the trap model is then described by the ME (79) discussed in 
section 4.2 in terms of the probability function $P(E, t)$ that specifies the probability that the system stays in a trap of energy $E$ at time $t$,

$$
\frac{\partial P(E, t)}{\partial t}=\sum_{E^{\prime}} P\left(E^{\prime}, t\right) Z\left(E \mid E^{\prime}\right)-\sum_{E^{\prime}} P(E, t) Z\left(E^{\prime} \mid E\right)
$$

The rates $Z\left(E \mid E^{\prime}\right)$ are assumed to be given by (82),

$$
Z\left(E \mid E^{\prime}\right)=\mathcal{W}\left(E \mid E^{\prime}\right) \rho(E)=\frac{\rho(E)}{\tau\left(E^{\prime}\right)} ; \quad \int_{0}^{\infty} \rho(E) d E=1
$$

where we have identified $\Omega\left(\mathcal{F}^{\prime}, T\right) \equiv \rho\left(E^{\prime}\right)$ and where the bare rate $\mathcal{W}\left(E \mid E^{\prime}\right)=$ $1 / \tau\left(E^{\prime}\right)$ has the dimensions of a frequency. Note that this bare rate only depends on the energy of the departure trap but not on the energy of the arrival trap. Other versions of the trap model include a dependence on the energy on the arrival trap, see for instance [173]. Inserting (208) into (207) we obtain,

$$
\frac{\partial P(E, t)}{\partial t}=\omega(t) \rho(E)-\frac{P(E, t)}{\tau(E)}
$$

where

$$
\omega(t)=\int_{0}^{\infty} d E^{\prime} \frac{P\left(E^{\prime}, t\right)}{\tau\left(E^{\prime}\right)} .
$$

The rates (208) satisfy detailed balance if $P^{\mathrm{eq}}(E) \propto \rho(E) \tau(E)$, where $\lim _{t \rightarrow \infty} P(E, t)=P^{\mathrm{eq}}(E)$. The Bouchaud trap model (BTM) [40] is described by the distribution $\rho(E)=\left(1 / T_{\mathrm{g}}\right) \exp \left(-E / T_{\mathrm{g}}\right)$. The static formulation of such a model corresponds to the random-energy model (REM) of Derrida [174, 175]. Other trap models have considered a Gaussian distribution of energies [39, 176]. However, the main interest of the model proposed by Bouchaud is the existence of a critical temperature $T_{\mathrm{g}}$ where the distribution $P^{\mathrm{eq}}(E)$ ceases to be normalizable. In general, for any distribution $\rho(E)$ the temperature $T_{0}$ that marks the onset of the nonnormalizability of $P^{\mathrm{eq}}(E)$ is given by [177,

$$
\frac{1}{T_{0}}=-\lim _{E \rightarrow \infty} \frac{\log (\rho(E))}{E}
$$

the BTM corresponding to the case $T_{0}=T_{\mathrm{g}}$. Let us note that, in the trap model, energies are not extensive but finite. Comparing the BTM with the REM, where energies are extensive, we observe that the finite $T$ dynamics in the Bouchaud model occurs in a range of finite energies around $E_{0}$, the value at which the energy freezes in the REM below $T_{\mathrm{g}}$. The same exponential distribution of states, over a finite free-energy interval, is found in the SK model 48, 35.

Dynamics in the trap model has been exhaustively investigated in many works. In particular, it offers a rather good explanation of magnetic relaxation phenomena observed in spin-glasses [40, 178, 179, viscosity anomalies in glasses 177, 176, 180] and, more recently, it has been used as a test model to check whether FDT violations are well described by the Ansatz (91) and whether FD plots are meaningful [54, 55. Correlation and response functions can be defined in the BTM by assigning magnetizations to the different traps as is done to analyze the statics of the REM. The effect of the magnetic field on the traps is to modify the escape time by the relation, $\tau_{h}(E)=\tau_{0} \exp ((E+m h) / T)=\tau(E) \exp (m h / T)$. The resulting FD plots have been analyzed by Sollich and coworkers [54, 55. As remarked in reference [54, 55, equal time correlations can be unbounded so proper FD plots are constructed from the raw 

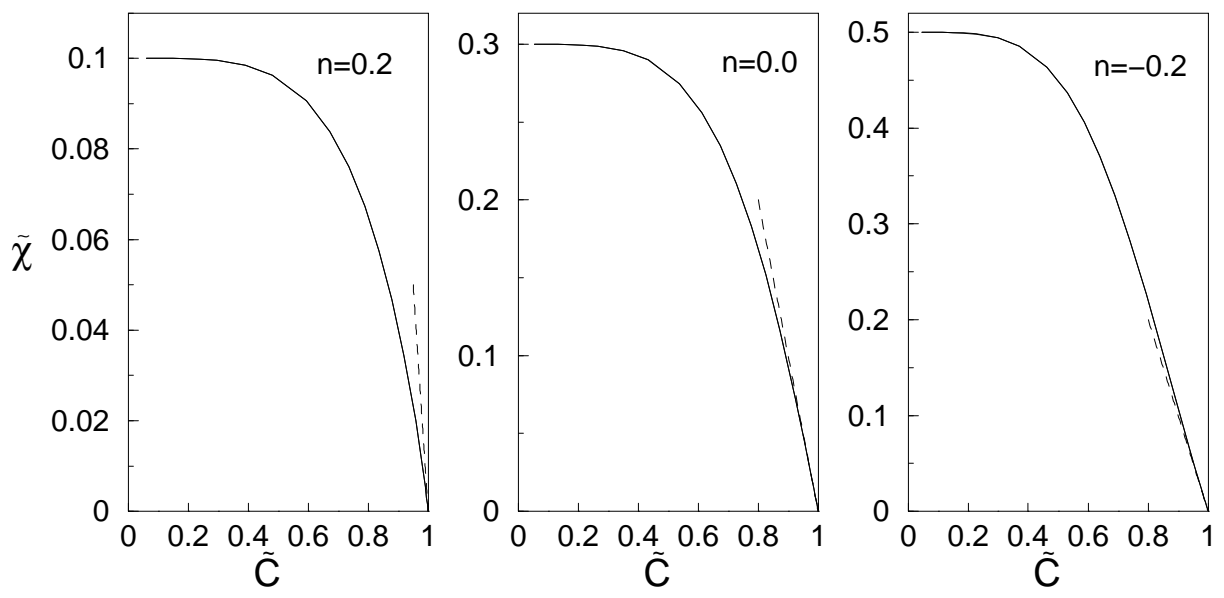

Figure 9. Normalized FD plots (104) for the BTM for a Gaussian distribution of trap magnetizations with zero mean and variance $\Delta(E)=\exp (n E / T)$ for different values of $n$ and $T=0.3\left(\tau_{0}=T_{\mathrm{g}}=1\right)$. For each $n$ times shown are $10^{6}, 10^{7}$ which are indistinguishable since the limiting FD plot has been reached. Note, however, that a temperature factor has been absorbed in $\tilde{\chi}$ in such a way that the slope is -1 at equilibrium (dashed line). From [54 55].

plots (102) by normalizing correlations and IRF by the equal times correlation at the later time $C_{A, B}(t, t)$ as described in (104). The ME (209), modified to include the effect of the field, is described by a probability distribution $P(E, m, t)=P(E, t) \sigma(m \mid E)$ where $\sigma(m \mid E)$ is the probability that a trap of depth $E$ has magnetization $m$. This probability is assumed to be a Gaussian parametrized by its mean $\bar{m}(E)$ and variance $\Delta^{2}(E)$. As there is no specific meaning attached to the observable $m$ one can think of the two functions $\bar{m}(E), \Delta^{2}(E)$ as describing different class of observables. Therefore, observable dependence in the BTM refers to dependence of the FD plots on the choice of these functions. The following cases have been considered [54, [55]: $\bar{m}(E)=\exp (n E / 2 T), \Delta^{2}(E)=0$ or $\bar{m}(E)=0, \Delta^{2}(E)=\exp (n E / T)$ with $n>T-1$ in both cases. Figure 9 shows some typical FD plots.

Three are the main results of this study: 1) The FD plots strongly depend on the average and the variance of the Gaussian distribution, therefore the FDR and the effective temperature are observable dependent; 2) Most importantly, for a given choice of observables the effective temperature (132) smoothly changes along time within a given time sector. In fact, in the trap model the scaling $t / t_{w}$ is fulfilled in the glassy phase $T<T_{\mathrm{g}}$ but two straight lines (typical of two-timescales glassy systems) are not observed in the FD plots; 3) For all observables $X_{\infty}=0$, see (231), supporting the conjecture that this quantity is indeed universal and may have some physical meaning (see section 6.6).

Among these results 2) seems particularly interesting. Why FD plots do not display the characteristic two-step form of relaxational systems with two-timescales? Still for observables with zero mean and finite (but $E$ independent) variance where $C(t, t)=\Delta^{2}$, i.e. for observables that can be considered neutral (see discussion in section 4.4), the one-step shape of the limiting FD plot is absent. The origin of this discrepancy is presently unknown and the finding of a trap model that shows a two 
straight-lines FD plot remains an interesting open problem $\ddagger$. Let us finish these considerations by noting that FD plots, such as the one depicted in Figure 9 are more characteristic of systems with full RSB where $X(C)$ is a non-trivial function. Quite interestingly, it has been shown [179] that the BTM at the critical temperature $T=T_{\mathrm{g}}$ has correlations that do not fulfill the simple $t / t_{w}$ scaling but a more complicated dependence with many time sectors and ultrametricity. However, the resulting FD plot at criticality shows only very small deviations from the equilibrium behavior $X=1$ being very similar to the FD plot observed in the Ising chain [182] (see section 6.6).

Just before ending this section, let us remind that these results have been endorsed by considering the corresponding driven version of the BTM introduced in [183, 184] in the context of rheology. In this case, as explained in section 7.4.1 the equivalent of the waiting time is the inverse of the shearing rate. In the stationary state TTI is satisfied but FDT violations persist. For the driven model [185], as well as for the purely relaxational model, the same relationship between correlations and responses holds and the two models the non-driven and the driven one) show similar behavior.

\subsection{Models with entropy barriers}

Many of the results described in the previous subsections deal with disordered models with a complex thermodynamics. However, many aspects of the violations of the equilibrium FDT as well as the existence of an QFDT can be investigated in the framework of simpler models that are exactly solvable but still retain the key ingredients for the emergence of these new properties. In turn, this can help to identify the basic ingredient that any sensible general theory must incorporate.

The scope if this section is mainly illustrative as it intends to present some of the basic ideas of previous section 4.2 applied to very simple examples. We will focus our discussion on the oscillator (OSC) and the Backgammon (BG) models. A comprehensive account of other results about these models can be found in a recent review 15]. Both models have a simple energy landscape and dynamics is determined by the presence of entropy barriers. The intuitive meaning of this term is the following. In general, relaxation in glassy models proceeds by activated jumps over energy barriers that allow the system to escape from a given trap after reaching a barrier of height $B$, the typical time needed in this process being given by the Arrhenius law $\tau \sim \exp (B / T)$. Activated dynamics is strongly temperature dependent, and for $T=0$ the dynamics is completely arrested, the system remains trapped forever and correlations do not decay to zero anymore (ergodicity is broken). When the dynamics is dominated by entropy barriers the relaxational mechanism is different. The system escapes from a trap through a process which involves a timescale $\tau$ which does not directly depend on the temperature but, for instance, on the typical energy $E$ of the trap itself, $\tau(E)$ which usually is a decreasing function of the energy $E$ (see Figure [10) $\S$. At $T=0$ relaxation is not arrested but proceeds slower as the energy decreases. This type of dynamics is sometimes called marginal dynamics [186] as the system wanders around saddle point configurations,

$\ddagger$ After completion of this work, it has been shown that the influence of the dependence of the perturbed rates in a field (upon the observable value taken at the arrival trap) are crucial to get well defined limiting FDRs and effective temperatures [181]

$\S$ Now we assume the standard convention for energies being negative rather than positive as in the trap model. 


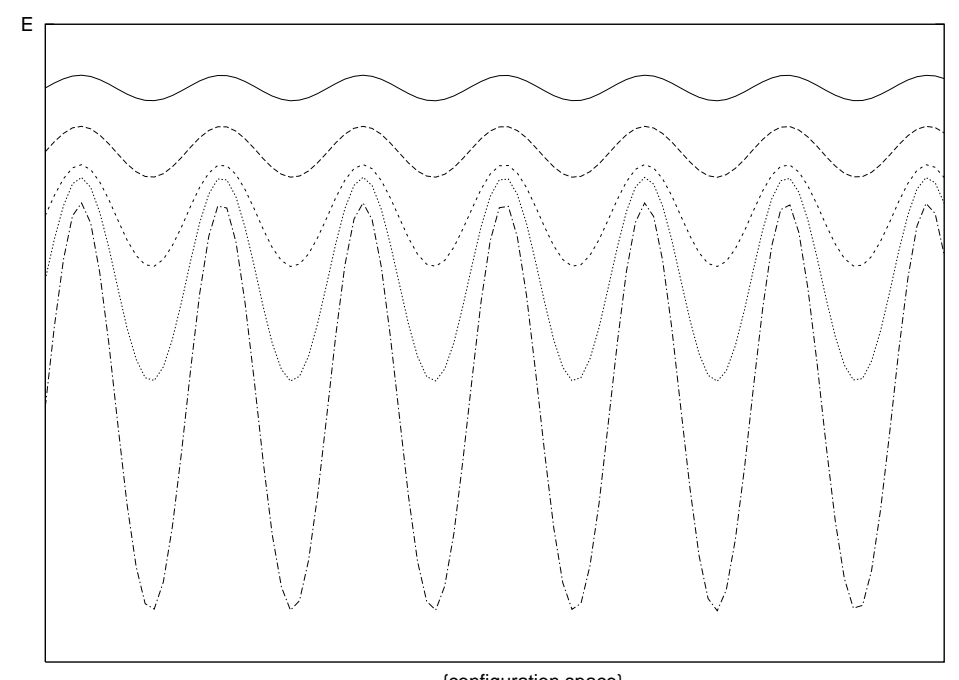

\{configuration space\}

Figure 10. A typical energy landscape in a glassy model determined by the presence of entropy barriers. The effective barrier $\log (\tau(E))$ increases as $E$ decreases. From 187.

hence is never arrested. Of course, the temperature dependence of the relaxation time in entropic models can appears as effectively activated: at finite (but low) temperatures the maximum value of $\tau(E)$ corresponds to the lowest energy reached, i.e. $\tau\left(E_{\text {eq }}(T)\right), E_{\text {eq }}(T)$ denoting the equilibrium energy. The temperature dependence of the relaxation time in equilibrium $\tau\left(E_{\mathrm{eq}}(T)\right)$ is activated in most entropy barrier models. A phenomenological description of these entropy models has been introduced by Barrat and Mezard [173] who have generalized the BTM to the case where the distribution of trapping times is itself a time-dependent function. The oscillator and Backgammon models described in this section are microscopic versions of this entropic trap model. According to the scenario presented in section 4.2 the role of entropy appears to be important as relaxation in many glassy systems is accompanied by the emergence of a non-equilibrium microcanonical ensemble which determines fluctuations and responses in the aging state, leading to the existence QFDT and an effective temperature. This fact suggests that a deep comprehension of the glassy dynamics in exactly solvable entropy barrier models can be a first step toward grasping the leading aspects behind the behavior of more realistic systems, where both entropy and energy barriers simultaneously intervene.

6.5.1. Oscillator models We begin our discussion by describing the OSC model in its simplest version. A review of some results can be found in 68, 188, 15]. Originally, oscillator models where introduced indirectly in the analysis of the Monte Carlo dynamics of the spherical Sherrington-Kirkpatrick model, which can be mapped to a set of disordered harmonic oscillators [189, 190. The OSC model is obtained by simplifying the previous one to an ensemble of identical harmonic oscillators 191. The OSC model is defined by the following energy function

$$
E=\frac{K}{2} \sum_{i} x_{i}^{2}
$$


where the $x_{i}$ are real-valued displacement variables for the $N$ oscillators and $K>0$ is the Hooke constant. The equilibrium properties are trivial due to the absence of interactions, but the Monte Carlo dynamics couples the oscillators in a nontrivial way. Moves are proposed according to the following rule,

$$
x_{i} \rightarrow x_{i}^{\prime}=x_{i}+\frac{r_{i}}{\sqrt{N}}
$$

where the $r_{i}$ are Gaussian random variables with zero mean and variance $\Delta^{2}$. Moves are accepted according to the usual Metropolis rule. Each move is a parallel update of the whole set of oscillators. Both the energy function (212) and the dynamics (213) are invariant under rotations in the $N$-dimensional space of the $x_{i}$. This symmetry makes the dynamics exactly solvable, and many non-equilibrium quantities e.g., effective temperatures and FDT violations can be tackled analytically.

The OSC model is a classical model where the equilibrium entropy is given by $S(T)=\frac{1}{2} \log (T)$, thereby diverging when $T \rightarrow 0$ as expected for a continuous model (the ground state is a zero-measure state corresponding to the absolute global minimum of (212), i.e. the configuration $\left.x_{i}=0, \forall i\right)$. At $T=0$ only those moves that decrease the energy are accepted, therefore as the system approaches the global minimum the frequency of accepted moves (213) dramatically decreases. However, that frequency never vanishes so dynamics is never arrested. Dynamics slows down because phase space directions where energy decreases are exceedingly difficult to find. The full solution of the OSC model has been presented in [191].

The main physical quantity containing detailed information about the dynamical evolution is the probability density of energy changes $P(\Delta E)$. This quantity expresses the probability density that a proposed move (213) changes the total energy of the ensemble by the amount $\Delta E . P(\Delta E)$ was originally derived in [191] using standard integration tools. Here we present two other alternative derivations which help to understand the mechanisms behind slow relaxation.

The first method relies on the Gaussian form of the distribution while the second one uses a microcanonical argument to count the number of accessible configurations from a reference configuration with a given energy $E$. The first derivation is rather simple as the distribution for $\Delta E$ can be easily obtained. Indeed using (212) and (213)

$$
\Delta E=\frac{K}{\sqrt{N}} \sum_{i} x_{i} r_{i}+\frac{K}{2 N} \sum_{i} r_{i}^{2} .
$$

and the Gaussian property of $r_{i}$, it follows that $\Delta E$ has a Gaussian distribution. The mean and variance of the distribution are $M_{\Delta E}=\overline{\Delta E}=K \Delta^{2} / 2, \sigma_{\Delta E}=$ $\overline{(\Delta E)^{2}}-(\overline{\Delta E})^{2}=2 E K \Delta^{2} / N$, yielding [191,

$$
P(\Delta E)=\left(4 \pi e K \Delta^{2}\right)^{-\frac{1}{2}} \exp \left[-\frac{\left(\Delta E-\frac{K \Delta^{2}}{2}\right)^{2}}{4 e K \Delta^{2}}\right]
$$

where $e=E / N$ is the energy per oscillator.

The second method to derive (215) is based on a microcanonical computation. In Figure [1] we depict a schematic two-dimensional representation of the motion of a representative configuration in phase space. The reference configuration $\left\{x_{i}^{0}\right\}$ at a given time has an energy $E$ and lies on the spherical hypersurface of radius $R=\sqrt{2 E / K}$ (depicted as the point $P$ in the figure) with center at the origin $\left\{x_{i}=0\right\}$ (point $O$ in the figure). The smaller dashed circle represents the region of points accessible from $\left\{x_{i}^{0}\right\}$ according to the dynamics (213). All accessible points 


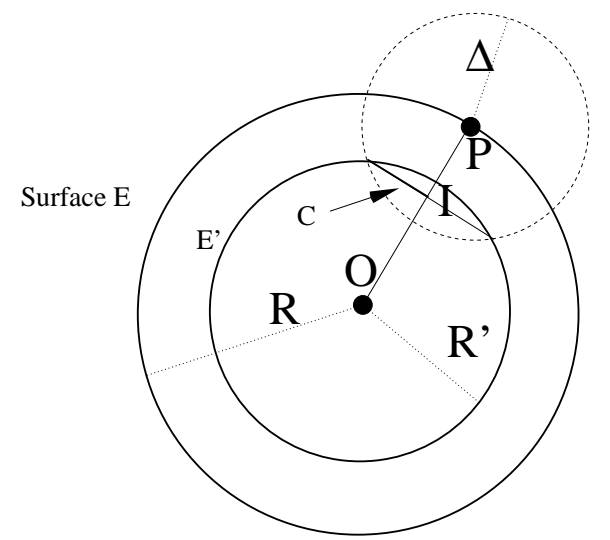

Figure 11. Geometrical construction to compute $P(\Delta E)$. The thick lines denote the departing and final energy hypersurfaces centered at $O$. The dashed circle indicates the hypersurface accessible from point $P$. The intersection region between the accessible hypersphere centered at $P$ and the final hypersurface of energy $E^{\prime}$ defines a hypersurface $I$ of radius $C$ (the radius is represented by a thick line). See the text for explanation.

$\left\{x_{i}\right\}$ satisfy $\sum_{i}\left(x_{i}-x_{i}^{0}\right)^{2}=\Delta^{2}$ i.e. lie at a distance $\Delta$ from $\left\{x_{i}^{0}\right\}$ which is the radius of the smaller dashed circle. The accessible configurations in a single move lie in a spherical hypersurface of dimension $N-2$ corresponding to the intersection of the hypersurface of energy $E^{\prime}$ and the smaller spherical hypersurface of radius $\Delta$. We call this region the intersecting region $I$ as shown in Figure 11] The final configurations contained in $I$ lie at a distance $R^{\prime}=\sqrt{2 E^{\prime} / K}$ to the origin $O$. The change in energy associated to this transition is $\Delta E=E^{\prime}-E$. The probability of this jump is therefore proportional to the surface of the intersection region, $P(\Delta E) \propto C^{N-2}$ where $C$ is the radius of the intersecting region. The computation of $C$ is quite straightforward as can be deduced from the triangle including the points $\mathrm{P}, \mathrm{O}$ as vertices and whose three sides are $R, R^{\prime}, \Delta$. In terms of $R, R^{\prime}$ and $\Delta$, the distance $C$ is given by the relation,

$$
C^{2}=\Delta^{2}-\frac{K}{8 E}\left(\frac{2 \Delta E}{K}+\Delta^{2}\right)^{2} .
$$

The surface $\Omega(E, \Delta E)$ corresponding to the region $I$ of radius $C$, relative to the energy $E$ of the reference configuration $\boldsymbol{x}^{0}$ is,

$$
\Omega(E, \Delta E) \propto C^{N-2}=\left[\Delta^{2}-\frac{K}{8 E}\left(\frac{2 \Delta E}{K}+\Delta^{2}\right)^{2}\right]^{\frac{N-2}{2}} .
$$

Using the fact that $E$ is extensive with $N$ this expression can be rewritten as,

$$
\Omega(E, \Delta E) \propto \exp \left[-\frac{\left(\Delta E-\frac{K \Delta^{2}}{2}\right)^{2}}{4(E / N) K \Delta^{2}}\right]
$$

which is proportional to the probability distribution (215).

The distribution $P(\Delta E)$ is depicted in figure 12 for different values of the energy. As $E$ decreases the number of moves with $\Delta E<0$ shrinks as the total area under the curve with $\Delta E<0$ decreases. From (215) the dynamical equations immediately follow by defining a Monte Carlo step as a collection of $M$ elementary moves (each elementary 


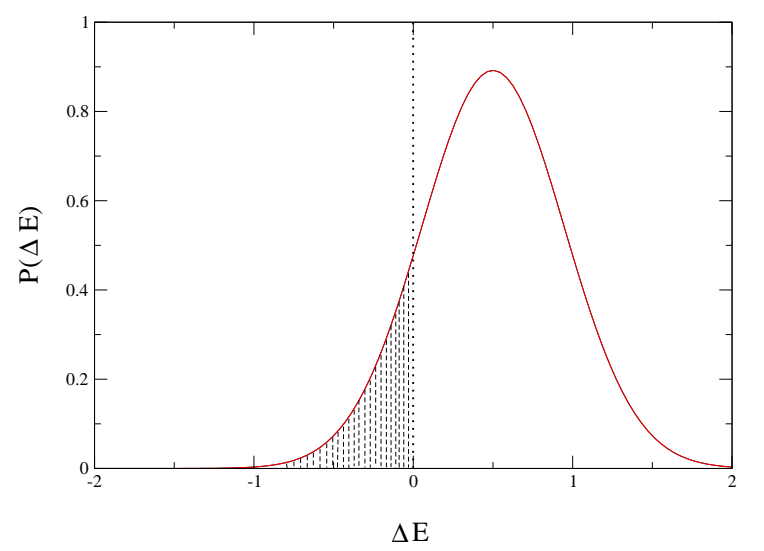

Figure 12. Probability density of the energy of the proposed moves for different values of the energy $E$ as defined in Figure 11

move corresponds to a global change of all oscillator coordinates as described in (213)). Because the average change of energy $\Delta E$ is finite in an elementary move and the total energy (212) is extensive, the number of moves $M$ in a Monte Carlo step must be proportional to $N$. For simplicity we will take $M=N$. In the limit $N \rightarrow \infty$, time becomes a continuous variable. This allows to write a closed equation for the energy $E$ and acceptance rate $A$, i.e., the average number of accepted moves in a Monte Carlo step,

$$
\begin{aligned}
& \frac{\partial E}{\partial t}=\int_{-\infty}^{\infty} x P(x) W(x) d x \\
& \frac{\partial A}{\partial t}=\int_{-\infty}^{\infty} P(x) W(x) d x
\end{aligned}
$$

where $P(x)$ is the probability distribution (215) and $W(x)$ is the transition rate which ensures that detailed balance is obeyed. For instance, according to the Metropolis rule $W(x)=\min [1, \exp (-\beta x)]$ or in heat-bath $W(x)=1 /[1+\exp (\beta x)]$. At zero temperature the transition rate rules $W(x)$ converge to $W(x)=\theta(x)$. In this limit (219), (220) become

$$
\begin{aligned}
& \frac{\partial E}{\partial t}=\int_{-\infty}^{0} x P(x) d x \\
& A(t)=\int_{-\infty}^{0} P(x) d x
\end{aligned}
$$

As shown in Figure 12 the acceptance is given by the shaded area enclosed in the negative tail of the distribution. At zero temperature, according to (215), both the mean and the width of the Gaussian decrease as well as the shaded area in Figure 12 implying a systematic decrease of the acceptance rate.

We do not want to dwell here on all results one can learn by solving the dynamical equations (221), (222) (see 191, 108]). Interestingly, in the OSC model the FDR (131) for the magnetization $M=\sum_{i=1}^{N} x_{i}$ can be exactly computed at any temperature [191,

$$
T_{\text {eff }}(s)=2 E(s)+\frac{1}{f(s)} \frac{\partial E(s)}{\partial s}
$$


and the QFDT is satisfied. At $T=0$ in the large time limit one gets $T_{\text {eff }}(s)=2 E(s)$ plus subleading corrections. This result shows that FD plots are straight-lines starting at $C(s, s)=(2 E(s) / K), \chi(s, s)=0$ and finishing at $C=0, \chi=1 / K$. The relation between the effective temperature and the dynamical energy is exactly the same as the equilibrium relation given by the equipartition theorem. The aging system is in a quasistationary state where relations between dynamical quantities are formally the same as in equilibrium. This allows to define a time-dependent configurational entropy $S_{c}(E)$ through the relation (90),

$$
\frac{1}{T_{\text {eff }}(E)}=\frac{\partial S_{c}(E)}{\partial E} \quad ; \quad S_{c}(t)=S_{c}(E(t))=\frac{1}{2} \ln (E(t)) \quad .
$$

Until now we have discussed some of the analytical results obtained by solving the dynamical equations of the OSC model. However, an interesting question is the following: can we determine the value of the effective temperature from the sole evolution of the energy $E$ without having to analyze correlations and responses in the framework of the QFDT as described in (223)? Ideally we would like to apply the ideas presented in section 4.2 to identify the value of $T_{\text {eff }}$ solely from the off-equilibrium transition rates $Z\left(F \mid F^{\prime}\right)$. In that description dynamics proceeds by activated jumps over different states, whose dynamics is described by the free-energy ME (79). What are the states in the reduced description of the OSC model? As the energy landscape is a single parabolic well it appears that a reduced description is not possible. The clue to this question is easy to understand if one realizes that at zero temperature the acceptance of the dynamics goes to zero with time, therefore each time a proposed elementary move is accepted we can effectively consider that the system has jumped from one state to another, the typical time for this jump steadily growing with the time elapsed since the system was quenched. In this view, each state corresponds to a configuration and the reduced dynamics simplifies. Moreover, the free energy of the state is simply the energy of the corresponding configuration. In the asymptotic regime $(d E / d t) / E<<1$ where finite size effects are not important, i.e. $\Delta \ll \sqrt{2 E / K}$, the probability distribution describing the energy change after the first jump is given by,

$$
P(\Delta E) \sim \exp \left(\frac{\Delta E}{4 e}\right) \theta(-\Delta E)
$$

where we used (215) and expanded it around $\Delta E=0$ up to the linear order. Using relation (224) we can recast (225) in the following form,

$$
P(\Delta E)=\frac{1}{2 T_{\mathrm{eff}}(E)} \exp \left(\frac{\Delta E}{2 T_{\mathrm{eff}}(E)}\right) \theta(-\Delta E)
$$

showing that the statistics of energy jumps is an exponential with a width that directly depends on the effective temperature. This result has two consequences: 1) It shows that the OSC model is a microscopic version of the trap model proposed by Barrat and Mezard [173] 2) The effective temperature could be computed from the statistics of the first free-energy jumps among components (here corresponding to configurations).

Before finishing, let us note that a number of variants of the oscillator model have been considered, all sharing the feature that oscillators do not interact. For example, Nieuwenhuizen and Leuzzi [108, 192, 193, 194] have considered a model with spin variables in addition to oscillators. The new variables are discrete and used to mimic fast relaxational processes not contained in the original formulation; aging, slow relaxation in these models, can be still described in terms of entropy barriers. 
6.5.2. The Backgammon and urn models Another instructive model where relaxation is determined by entropic barriers is the Backgammon (BG) model [195. The model belong to a large class of models under the name of urn models where $N$ particles or balls are distributed among $M$ urns or boxes. The BG model is defined by the energy function,

$$
E=-\sum_{r=1}^{M} \delta_{n_{r}, 0}=-N_{\text {empty }}
$$

where $n_{r}$ stand for the occupancies for each box and $N_{\text {empty }}$ stands for the number of empty boxes. The model has different versions [196] according to whether particles are distinguishable (Maxwell-Boltzmann statistics) or not (Bose-Einstein statistics). The easiest way to compute the thermodynamic properties of the BG model is by expressing the partition function in terms of the occupancies $n_{r}$ rather than in terms of the set of boxes occupied by the particles. In the Maxwell-Boltzmann case, thermodynamics needs to be corrected by dividing the partition function by the usual $N$ ! term to avoid Gibbs' paradox. In the model with Bose-Einstein statistics this is not necessary. The dynamical rules of the BG model directly depend on the type of statistics considered. In the Maxwell-Boltzmann case, a departure box $d$ is selected with a probability proportional to the occupancy $n_{d}$ of that box and a new arrival box $n_{a}$ is selected with uniform probability. The proposed move is given by $n_{d} \rightarrow n_{d}-1, n_{a} \rightarrow n_{a}+1$ and accepted according to the standard Metropolis rule. Instead, in the Bose-Einstein case, the proposed move and the transition rate are the same as for the MaxwellBoltzmann case but both the departure and the arrival box $d$ and $a$ are selected with a uniform probability $1 / M$.

The resulting dynamics of the model is strongly dependent on the type of statistics considered, the interesting one corresponding to the Maxwell-Boltzmann case which displays a strong separation of time-scales. In what follows, although otherwise stated, we will concentrate in the analysis of the model with $M=N$.

The thermodynamics of the model is exactly solvable and, the entropy being given by $S(e)=\log (1+e)$ where $e=E / N$ denotes the entropy per box (or per particle). To analyze the dynamics from the perspective of the scenario described in section 4.2 we follow a similar reasoning as we did for the OSC model. Let us consider a system quenched down to $T=0$ and the aging regime reached in the asymptotic long-time regime where $(d E / d t) / E<<1$. In that limit, the system has a number of empty boxes $N_{\text {empty }}=-E$ and further decrease of that number by one unit $\Delta E=-1$ requires a time that exceedingly grows as $E$ decreases toward its minimum value $E=-N+1$ (all particles condensed into a single box) 197, 198. Therefore, as relaxation slows down, for a long time the system wanders through the constant energy surface by exchanging particles among occupied boxes. When a new box is emptied the energy decreases by one unit. As we did in the OSC model, also in the $T=0$ dynamics of the BG model each component $\mathcal{R}$ corresponds to a single configuration with free energy equal to the energy of that configuration.

The transition rate for going from a configuration of energy $E$ to a configuration of energy $E-1$ is sole function of the number of available configurations in the initial and final state,

$$
\frac{Z_{t}(E-1 \mid E)}{Z_{t}(E \mid E)}=\frac{\Omega(E-1)}{\Omega(E)}
$$


where the rate frequency $1 / t(E)$ associated to $Z_{t}$ (for its definition, see Section 4.2) has canceled out from the numerator and denominator in the left hand side of (228). As $E$ is extensive with $N$, and using (83) we obtain,

$$
\frac{Z_{t}(E-1 \mid E)}{Z_{t}(E \mid E)}=\exp \left(-\frac{\partial S(E)}{\partial E}\right)=\exp \left(-\frac{1}{T_{\text {eff }}(t)}\right)
$$

Equation (229) provides a way to estimate the effective temperature by looking at the number of moves required for a move to decrease the energy by one unit. Inverting (229) yields

$$
T_{\text {eff }}(t)=\left(\ln \left[\frac{Z_{t}(E \mid E)}{Z_{t}(E-1 \mid E)}\right]\right)^{-1}=\frac{1}{N^{*}(t)}
$$

Therefore the ratio $Z_{t}(E \mid E) / Z_{t}(E-1 \mid E)$ is simply given by the inverse of the number of accepted moves $N^{*}$ required to decrease the energy by one unit. We stress that $N^{*}$ is not the number of MC steps but rather the number of accepted elementary moves with $\Delta E=0$ required until a move with $\Delta E=-1$ is found. This number $N^{*}$ is independent of the size of the system. Note also that the rate of rejected moves (which gives the acceptance) does not enter into the expression of the effective temperature but rather into the value of the typical timescale $t(E)$ associated to the jump. This is an essential ingredient required in any "quasi-equilibrium" or microcanonical description of the relaxation. The value of the effective temperature at a given time only depends on the number of accessible states with lower free energy (energy in the present model) and not on the time necessary to escape from that state. This expression is very amenable to numerical calculations.

Note that the energy levels in the BG model are discrete, therefore the relation (226) cannot be applied. However, one can consider a non-degenerate disordered BG model where boxes are assigned different energies [187]. In this case the distribution $P(\Delta E)$ has been derived and shown to have a exponential time-dependent behavior characteristic of trap models. However, the computation of the effective temperature in that model, and the verification of (226) has not yet been done.

The Backgammon model as well as some of its variants has been solved by the technique of the generating function [199, 196, 200, 201, 202, 203, 204, 205. A discussion of some of the main results and analytical techniques has been presented in 15. The origin of the effective temperature as derived from the QFDT remains yet not completely understood. No neutral observable has been yet identified in the BG model. Although all studied observables show that the QFDT (94) is verified in a onetimescale scenario, there seem to be different effective temperatures depending upon the observable considered [202, 204, 206]. On the other hand, none of the different effective temperatures associated to these observables appears to be linked to the effective temperature obtained within the adiabatic approximation [199. An interest variant of urn models are zeta urn models introduced in the context of quantum gravity that show a finite-temperature Bose-Einstein condensation transition [207, 208, see section 6.6

\subsection{Ferromagnetic models at criticality}

A new emerging line of research related to the mainstream of researchs on FDT violations in glassy systems, is the study of FDT violations in critical points. In critical points the relaxation time diverges and one can investigate, for instance through field 
theoretical methods, the time-dependence of correlations, responses and the resulting FDR (911) in the asymptotic regime $s \rightarrow \infty$. These type of investigations are not different of those undertaken in the study of coarsening behavior in ordered phases. The main difference between the slow dynamics in a critical point and coarsening is that, in the former case, critical slowing down is at the origin of the slow dynamics. Interfaces have no stiffness tension and their motion is subdiffusive (or diffusive) and only consequence of the curvature of the interface. Growing domains are not islands of up or down spins but regions of spatially and temporally correlated spins of zero net magnetization. At $T_{\mathrm{c}}$ dynamics is described by the renormalization-group dynamical equations of the corresponding finite-temperature fixed point. However, in coarsening systems below $T_{\mathrm{c}}$ activated processes are important and interfaces have non-zero tension as they separate domains of up and down spins. Competition between the curvature of the surface and its tension leads to different growth laws, described by the zero-temperature fixed point. Because the origin of slow dynamics at $T_{\mathrm{c}}$ is different than in standard glassy systems, the main Ansatz (91) may not hold. An indeed, many studies of the FDR at criticality reveal that $X(t, s)$ is not a single function of the correlation $C(t, s)$.

One among the first studies of ferromagnetic models at criticality is the Ising chain solved by Glauber in 1963 209. Strictly speaking the dynamics of this model is that of a coarsening model as the system orders at the critical point $T=0$ where the magnetization discontinuously jumps from 0 (for $T>0$ ) to 1 (at $T=0$ ), i.e. the critical exponent $\beta$ vanishes. We will see below that the ferromagnetic Ising chain has some peculiar properties. Starting from a random initial configuration the coarsening dynamics at $T=0$ has long been studied in [210] and revisited in 211. In the asymptotic long-time limit, the aging part of the two-times correlation functions scales like $C_{\mathrm{ag}}(t, s) \sim F(L(t) / L(s))$ (252) with $L(t) \sim t^{1 / 2}$ corresponding to a diffusive process of the interfaces (see section 7.3 ). More recently, and nearly at the same time, the FDR has been analytically computed 212, 182] for a random staggered perturbation finding $X(C)=1 /\left[2-\sin ^{2}(\pi C / 2)\right]$ in agreement with the Ansatz (911). Or, in terms of times, $X(t, s)=(1 / 2)(1+s / t)$ showing that $X \rightarrow 1 / 2$ if $t \rightarrow \infty$ or $C \rightarrow 0$. This last result coincides with that found in the random walk or in the Gaussian model [170, all models characterized by a diffusive dynamics. This has led to the proposal [205] 213] that, in systems at criticality, the limiting value $X(t, s)$ for $t \rightarrow \infty$ is a universal quantity,

$$
X_{\infty}=\lim _{s \rightarrow \infty} \lim _{t \rightarrow \infty} X(t, s) .
$$

This conclusion has been substantiated by the exact solution of the ferromagnetic spherical model in general $d$ dimensions [205, 213. The authors have noticed how $X_{\infty}$ is only a function of amplitude ratios describing the leading scaling behavior of correlations and responses. This conclusion is endorsed by the following result: $X_{\infty}=1 / 2$ for $d>d_{\mathrm{ucd}}=4$ and $X_{\infty}=1-2 / d$ for $2<d<d_{\mathrm{ucd}}=4$ where $d_{\mathrm{ucd}}$ stands for the upper critical dimension (below $d=2$ there is no finite $T$ transition in the model). Interestingly, for $d=2$ the FDTR $X(C)$ has a non-trivial form 214], similar to what is found for the ferromagnetic Ising chain. Numerical results in the Ising model show that $X_{\infty} \simeq 0.26$ in $2 \mathrm{~d} \|$ and preliminary results in $3 \mathrm{~d}$ give $X_{\infty} \simeq 0.4$. In figure 13 we show the the IRF corresponding to the TRM susceptibility (98) as function of $C$ in the $2 \mathrm{~d}$ Ising model at the critical point. The general scenario about

$\|$ After completion of this review we learned from recent results finding a slightly different value $X_{\infty} \simeq 0.34$ in $2 \mathrm{~d}$ [215]. More work is necessary to accurately estimate that number. 


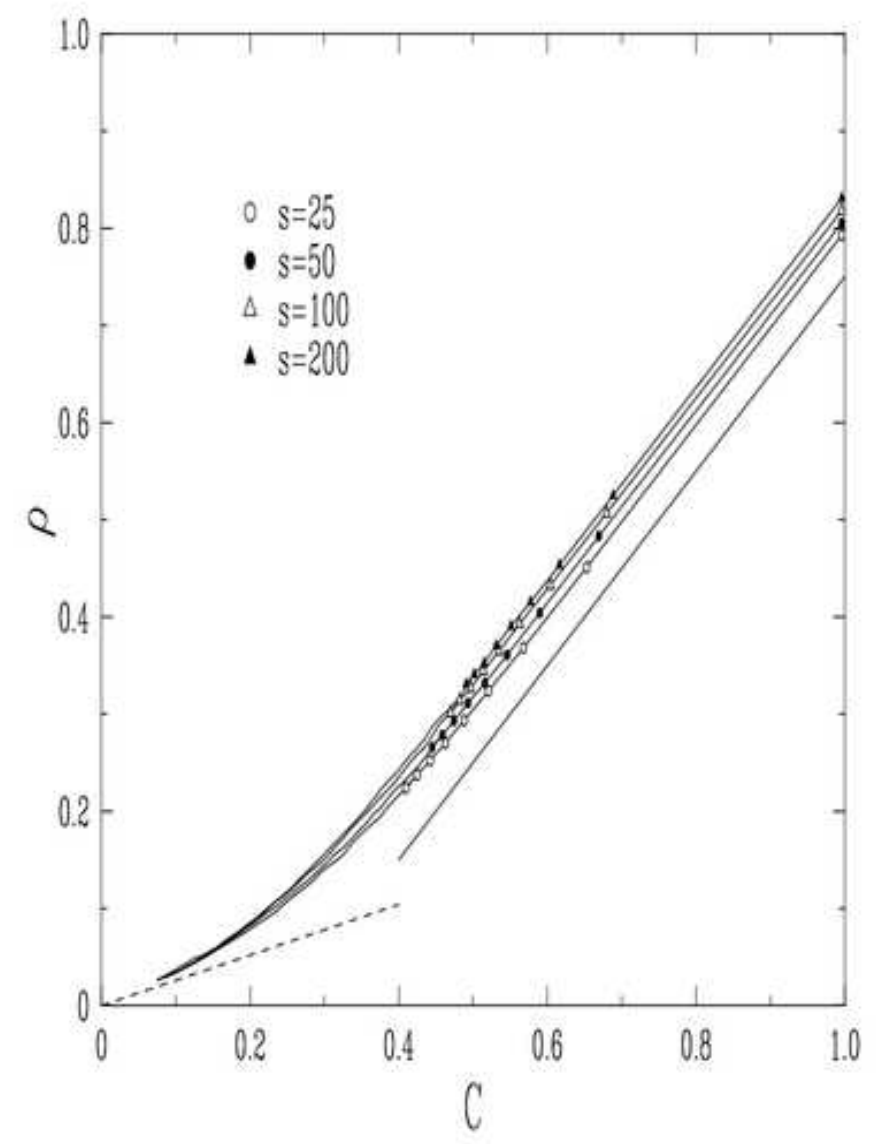

Figure 13. IRF 98 corresponding to the thermoremanent magnetisation in the $2 \mathrm{~d}$ Ising model at criticality. The full and broken line correspond to the quasi stationary regime $X=1$ and $X_{\infty}=0.26$ respectively. From 205].

ferromagnetic models at criticality is as follows 213. Let us consider a system that is quenched from a random configuration to a temperature below $T_{\mathrm{c}}$. If $\tau=t-s \ll s$ the system is in equilibrium, TTI holds and FDT is not violated $(X(t, s)=1)$. However, if $t / s \sim \mathcal{O}(1)$ the system ages, and both TTI and FDT are violated. A solution for the correlation $C_{\mathrm{ag}}(t, s)$ and response $R_{\mathrm{ag}}(t, s)$ that matches the intermediate regime between the stationary $X=1$ and the aging regimes is given by,

$$
C_{\mathrm{ag}}(t, s)=m_{\mathrm{eq}}^{2} \widehat{C}\left(\frac{t}{s}\right), \quad R_{\mathrm{ag}}(t, s)=s^{-a-1} \widehat{R}\left(\frac{t}{s}\right)
$$

where $a>0$ is a coarsening exponent. From (232) $X(t, s) \sim s^{-a} / m_{\text {eq }}^{2}$, therefore $X \rightarrow 0$ for coarsening systems where $m_{\text {eq }}$ is finite. The same expression is valid for the critical point but replacing $m_{\mathrm{eq}}$ by its time-dependence at $T_{\mathrm{c}}$. Using $m_{\mathrm{eq}} \sim\left(T_{\mathrm{c}}-T\right)^{\beta}, \xi \sim$ $\left(T_{\mathrm{c}}-T\right)^{-\nu}, t \sim \xi^{z}$ where $\beta, \nu, z_{c}$ are the, correlation length and dynamical exponents respectively. Substituting these relations into (232) and using $a_{c}=2 \beta / z_{c} \nu$ gives at 
$T_{\mathrm{c}}$

$$
C_{\mathrm{ag}}(t, s)=s^{-a_{c}} \widehat{C}\left(\frac{t}{s}\right), \quad R_{\mathrm{ag}}(t, s)=s^{-a_{c}-1} \widehat{R}\left(\frac{t}{s}\right)
$$

leading to $X(t, s)=\hat{X}(t / s)$. This result has two consequences: 1) for $t \rightarrow \infty$, $X(t, s) \rightarrow X_{\infty}$ and 2) only for $a_{c}=0$, according to the left expression in (233), $X(t, s)$ can be expressed as sole function of $C_{\mathrm{ag}}$. This is the case of the aforementioned Ising chain where the Ansatz (91) is valid because $\beta=0$. It has been suggested [216] that $R_{\text {ag }}(t, s)$ covariantly scales under conformal transformations of time leading to specific predictions for the scaling function $\widehat{R}$. An important implication of the result $a_{c}=0$ is that the aging part of the IRF (97) does not vanish in the asymptotic long-time limit. As noted in 217 the non-vanishing of the aging part of IRF in the large $s$ limit is related to the failure of the scheme that links static and dynamical properties [160] 161 in the Ising chain. In fact, as discussed in section 6.2.2 the stochastic stability property links the equilibrium $P(q)$ with the behavior of IRF,

$$
P(q)=-\left.T \frac{d^{2} \chi(C)}{d C^{2}}\right|_{C=q}=\left.\frac{d X(C)}{d C}\right|_{C=q}
$$

where we have used (100). In the Ising chain $P(q)=\delta(q-1)$. Equation (234) is not fulfilled in the Ising chain as can be easily checked inserting the result $X(C)=1 /\left(2-\sin ^{2}(\pi C / 2)\right)$ (discussed in the paragraph preceding (231) $)$. As soon as $T_{\mathrm{c}}$ is finite $(d>1), X(C)=\theta\left(C-m_{\mathrm{eq}}^{2}\right)$ for $T<T_{\mathrm{c}}$ and (234) is again satisfied. Despite its simplicity, the Ising chain appears to be an interesting solvable example that allows to check many results. For instance, observable independence has been also recently addressed [55] showing that $X_{\infty}=0$ for a large class of observables. Recent progress in the study of the FDR at criticality has been achieved by Calabrese and Gambassi [218, 219] who have considered the FDR in momentum space $X_{\vec{q}}(t, s)$. The study of $O(N)$ models using field-theoretical techniques yields estimates for the value of $X_{\infty}$ (231) in an $\epsilon=4-d$ expansion. Two-loops computations 219 give, for the Ising case $N=1$, the following values: $X_{\infty}(3 d)=0.429(6), X_{\infty}(2 d)=0.30(5)$ compatible with the results obtained from numerical simulations [205] 215].

Other studies of models at criticality include the XY model with a KosterlitzThouless transition [220]. This model has a low-temperature phase where correlation functions decay algebraically, therefore correlations are critical below $T_{\mathrm{c}}$. A failure of the stochastic stability property, similar to that reported in the Ising chain, appears in the $2 \mathrm{~d} X Y$ model at low temperatures where the density of vortices increases.

Finally, let us comment on results for zeta-urn models that have confirmed the validity of the relation (231) [207, 208, 15]. Zeta urn models show a quite rich phase diagram described in terms of the density $\rho$ of balls (i.e. the number of balls divided by number of boxes) and the temperature. There is a critical line $\rho_{c}(\beta)$ that separates a fluid regime $\left(\rho<\rho_{c}(\beta)\right)$ from a condensed regime $\left(\rho>\rho_{c}(\beta)\right)$ with glassy dynamics. Along the so called regular part of the critical line $(\beta>3) X_{\infty}=\frac{\beta+1}{\beta+2}$ is temperature dependent. This number lies in the interval $[4 / 5,1]$ quite far from the values $X_{\infty}<1 / 2$ found in ferromagnets. In the condensed phase $X_{\infty} \rightarrow T^{1 / 2}$ at low temperatures. It vanishes at $T=0$ as in coarsening systems, however the condensation dynamics in urn models is totally inhomogeneous in contrast to the homogeneous character of coarsening in ferromagnets. 


\section{QFDT: the numerical evidence}

Computer simulations have the great advantage, over the real experiment, of direct access to the microscopic level, even if only relatively small timescales and system sizes can be studied. Although this can be a serious limitations, the true fact is that the numerical study of aging phenomena and FDT violations has been successfully done during the last years for several systems.

\subsection{Structural glasses}

Using ideas developed in the field of spin glasses, many conjectures have been formulated concerning the structure of the phase space of glassy systems. However, obtaining direct information, either from experiments or from numerical simulations, is a difficult challenge. Relaxation times in a glass are so long as to preclude equilibration within experimental times. Numerical or experimental exploration of the phase space in these systems is therefore necessarily incomplete. The increase in computational power and the recent developments in the theory of disordered systems has pushed forward an approach which should not suffer from these limitations. The idea, which was actively developed in the study of spin glasses, is that relevant information on the phase space structure should be hard-encoded into the non-equilibrium dynamics of glassy systems.

According to the conjecture of the similarity between structural glasses and some spin-glass models, $X(C)$ for structural glasses is a two valued function with $X(C)=1$ at short times, and $X(C)=m<1$ in the long-time aging regime. This scenario has been largely studied using numerical simulations.

In a numerical investigation of aging effects not only the waiting time must be changed over several order of magnitude, but for a given waiting time the subsequent dynamics must be studied over a long time. For these reasons aging phenomena have been studied for models that are simple enough to be simulated over long times, but at the same time still catch the essential features of real glasses. Moreover, to maintain the systems in a non-equilibrium state for very long times, crystallization must be strongly inhibited. This is obtained either with a particular choice of interaction potential parameters or by adding a (small) extra term in the potential. In the following we discuss results obtained for several models.

\subsubsection{Mixtures of soft particles of different sizes 221, 222, 223, 224, 225, 226.}

This system consists of $N$ particles half of which are of type $A$ and half of type $B$ interacting via the Hamiltonian

$$
\mathcal{H}=\sum_{i<j}\left(\frac{r_{i}+r_{j}}{\left|\boldsymbol{x}_{i}-\boldsymbol{x}_{j}\right|}\right)^{12}
$$

where the radius $r_{i}$ depends on the type of particle. It is known that the choice of two different types of radius such that $r_{B} / r_{A}=1.2$ prevents crystallization and the system can be brought into a glassy state.

Due to the simple scaling of the potential, the thermodynamic quantities depend only on $\Gamma=\rho \beta^{4}$, where $\rho$ is the density which can be taken equal to one. This model presents a glass transition at about $\Gamma_{c}=1.45$ [222. In figure 14] we report the response 


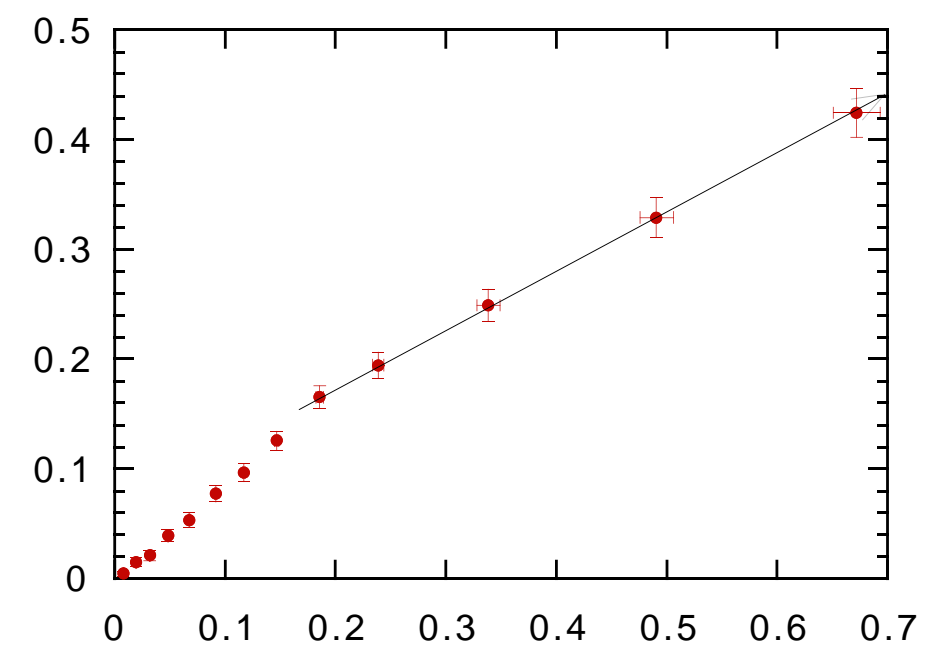

Figure 14. $\chi$ versus $\beta \Delta$ at $\Gamma=1.6$ for $t_{w}=t_{w}=8192$. and $t_{w}=2048$. From [225].

of the particle to a force of strength $\epsilon$

$$
\chi\left(t_{w}+\tau, t_{w}\right) \simeq \frac{1}{N \epsilon} \sum_{i=1}^{N}\left\langle\boldsymbol{f}_{i} \cdot \boldsymbol{x}_{i}\left(t_{w}+\tau\right)\right\rangle
$$

where $\boldsymbol{f}_{i}$ is a random Gaussian vector of squared length equal to the space dimension $d$, versus the self-diffusion function

$$
\Delta\left(t_{w}+\tau, t_{w}\right)=\frac{1}{N} \sum_{i=1}^{N}\left\langle\left|\boldsymbol{x}_{i}\left(t_{w}+\tau\right)-\boldsymbol{x}_{i}\left(t_{w}\right)\right|^{2}\right\rangle
$$

The average is over different initial states at $t_{w}$ and realization of $\boldsymbol{f}$. Two linear regions with different slopes, one with $X(C)=1$ and one with $X(C)=m<1$ are clearly seen, in agreement with the two-timescales scenario. The dependence of $m$ with $T$ is well fitted by the spin-glass model prediction $m(T)=T / T_{\mathrm{c}}$, see figure 15 . Similar results have been obtained in monoatomic Lennard-Jones glasses [227, 228], see section (7.1.3).

\subsubsection{Lennard-Jones binary-mixtures 229, 230, 231, 232,}

The system consist of a mixture of particles of type $A$ and $B$ of equal mass $m$ interacting via a $12-6$ Lennard-Jones potential of the form

$$
V_{\alpha \beta}(r)=4 \epsilon_{\alpha \beta}\left[\left(\frac{\sigma_{\alpha \beta}}{r}\right)^{12}-\left(\frac{\sigma_{\alpha \beta}}{r}\right)^{6}\right]
$$

where $\epsilon_{\alpha \beta}$ and $\sigma_{\alpha \beta}$ depend on the particle pair type and are chosen to prevent crystallization. For a $80: 20$ mixture, and using $\epsilon_{A A}$ and $\sigma_{A A}$ as units of energy and length and $\left(m \sigma_{A A}^{2} / 48 \epsilon_{A A}\right)^{1 / 2}$ as the unit of time, these are $\epsilon_{A A}=1, \sigma_{A A}=1$, $\epsilon_{A B}=1.5, \sigma_{A B}=0.8, \epsilon_{B B}=0.5$ and $\sigma_{B B}=0.88$. The atomic dynamics of this model is well described by the mode-coupling theory with a critical temperature of $T_{\mathrm{c}}=0.435$ in reduced units. 


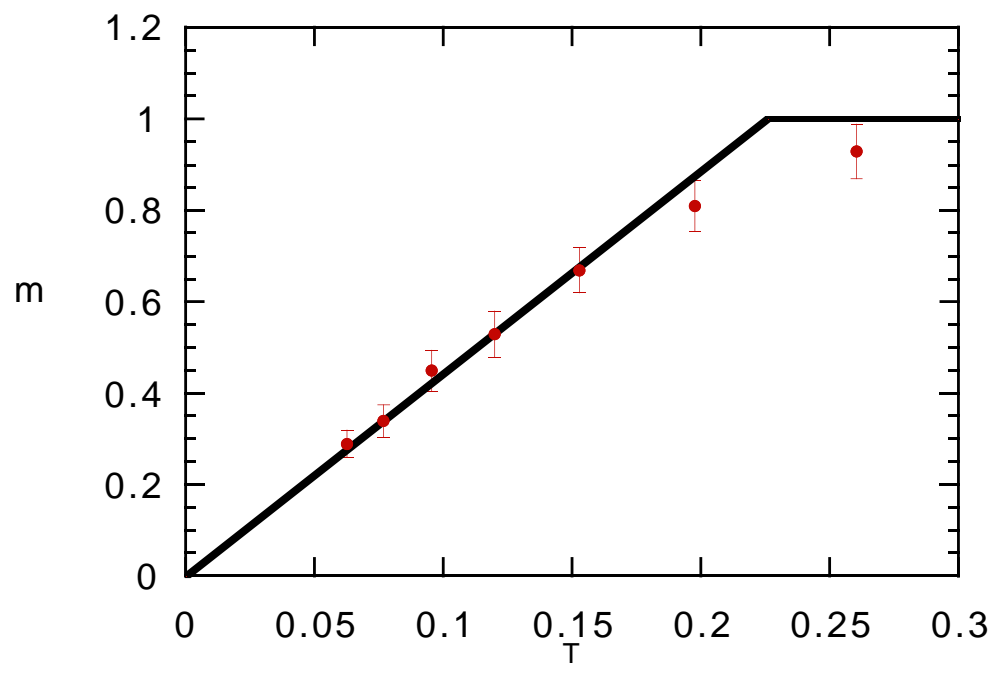

Figure 15. The quantity $m \equiv \frac{\partial \chi}{\partial \beta \Delta}$ as $t_{w}=2048$ as function of the temperature. The straight line is the prediction of the approximation $m(T)=T / T_{\mathrm{C}}$. From 225].

Typical FD plots numerically obtained by Barrat and Kob [233, 234, 235] are shown in figure 16. The correlation is given by the incoherent scattering function for a wave vector $\boldsymbol{k}$

$$
C_{k}\left(t_{w}+\tau, t_{w}\right)=\frac{1}{N} \sum_{j} e^{i \boldsymbol{k} \cdot\left[\boldsymbol{r}_{j}\left(t_{w}+\tau\right)-\boldsymbol{r}_{j}\left(t_{w}\right)\right]}
$$

while the response is measured by adding to the potential a term of the form

$$
\delta V=V_{0} \sum_{j} \epsilon_{j} \cos \left(\boldsymbol{k} \cdot \boldsymbol{r}_{j}\right)
$$

where $\epsilon_{j}= \pm 1$ with equal probability and $V_{0}<T$. Again a two-timescales scenario is clearly seen. Moreover the effective temperature in the slow regime where $m<1$ is in reasonable agreement with the glass transition temperature $T_{\mathrm{g}}$ of the system. $\dagger$

Thus the FDT is broken as the system fails to equilibrate, as expected from spin-glass models.

\subsubsection{Monoatomic Lennard-Jones systems [236, 237, 227, 228.}

The system consists of equal particles interacting via the potential $V=V_{\mathrm{LJ}}+\delta V$, where $V_{\mathrm{LJ}}$ is the usual $12-6$ Lennard-Jones potential (expressed in reduced units), and $\delta V$ is a many-body term that inhibits crystallization:

$$
\delta V=\frac{\alpha}{2} \sum_{\boldsymbol{q}} \theta\left(S(\boldsymbol{q})-S_{0}\right)\left(S(\boldsymbol{q})-S_{0}\right)^{2}
$$

where $S(\boldsymbol{q})$ is the static structure function, $\alpha=0.8$ and $S_{0}=1$. The sum is made over all $\boldsymbol{q}$ with $q_{\max }-\Delta<|\boldsymbol{q}|<q_{\max }+\Delta$, where $q_{\max }=7.12 \rho^{1 / 3}$ and $\Delta=0.34, \rho$ being the particle density.

$\dagger$ The glass transition $T_{\mathrm{g}}$ is defined as the temperature below which the system fails to equilibrate on the experimental time-scale. For structural glasses $T_{\mathrm{g}}$ is defined as the temperature at which the viscosity is equal to $10^{13}$ Poise or, equivalently, a relaxation time of $100 \mathrm{~s}$. 


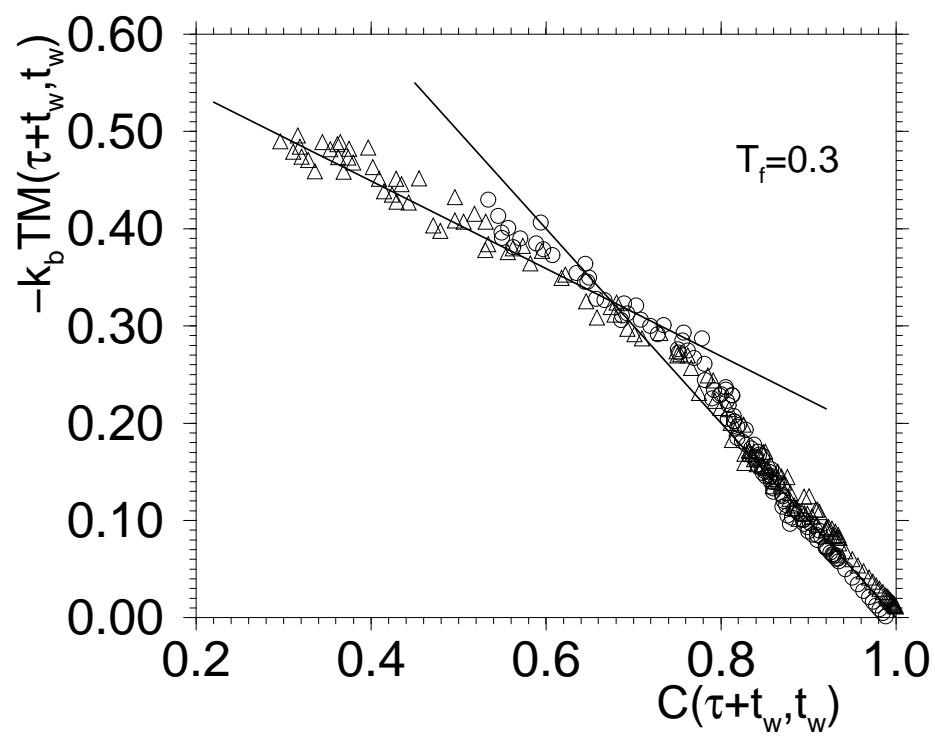

Figure 16. Parametric plot of the integrated response function $M\left(t_{w}+\tau, t_{w}\right)$ and the correlation function $C\left(t_{w}+\tau, t_{w}\right)$ for $k=7.25$. Final quench temperature $T_{\mathrm{f}}=0.3, t_{w}=1000$. Circles: $t_{w}=10000$. The straight lines have slopes -1.0 and $-0 . .45$. From 234 .

In figure 17] we report the parametric plot of the mean square displacement $\Delta$ and IRF in a crunch experiment. It is important to note that the temperature below which $m<1$ does coincide with the glass transition temperature of the system at the density reached after the crunch. This shows that the breaking of FDT does not depend on the initial state nor on the path followed in the $(T, \rho)$ plane, but only on the final (non-equilibrium) state to which the system is brought.

The FD plots for glass-forming liquids discussed here reveal the typical twotimescales (or 1RSB) scenario found in some spin-glass models. This supports the original Goldstein's idea [56] that the phase space of supercooled liquids is divided by high barriers into different valleys each with its own statistical properties. This picture has been recently confirmed by a a direct analysis of the motion of a glass forming liquids in terms of IS 62 .

In the right panel of figure 18 238 we show the temporal behavior of the average energy of minima for a binary mixture Lennard-Jones system visited in the nonequilibrium motion following a quench to a low temperature. As a consequence when the system is quenched from an high-temperature state to temperature $T$, the fast intra component degrees of freedom will quickly equilibrate with the thermal bath temperature T. Applying the condition of minimum free energy to the system, constrained to stay in components of depth $E_{\mathrm{IS}}$, allows to define an effective temperature

$$
T_{\text {eff }}\left(E_{\mathrm{IS}}, T\right)=\frac{\left(\partial / \partial E_{\mathrm{IS}}\right) F_{v}\left(T, E_{\mathrm{IS}}\right)}{\left(\partial / \partial E_{\mathrm{IS}}\right) S_{c}\left(E_{\mathrm{IS}}\right)}
$$

which reflects the non-equilibrium net heat flow from the system to the thermal bath [100]. This expression coincides with that proposed in [46] in the context of $p$-spin models, once the components are identified with the Thouless-Andreson-Palmer states 


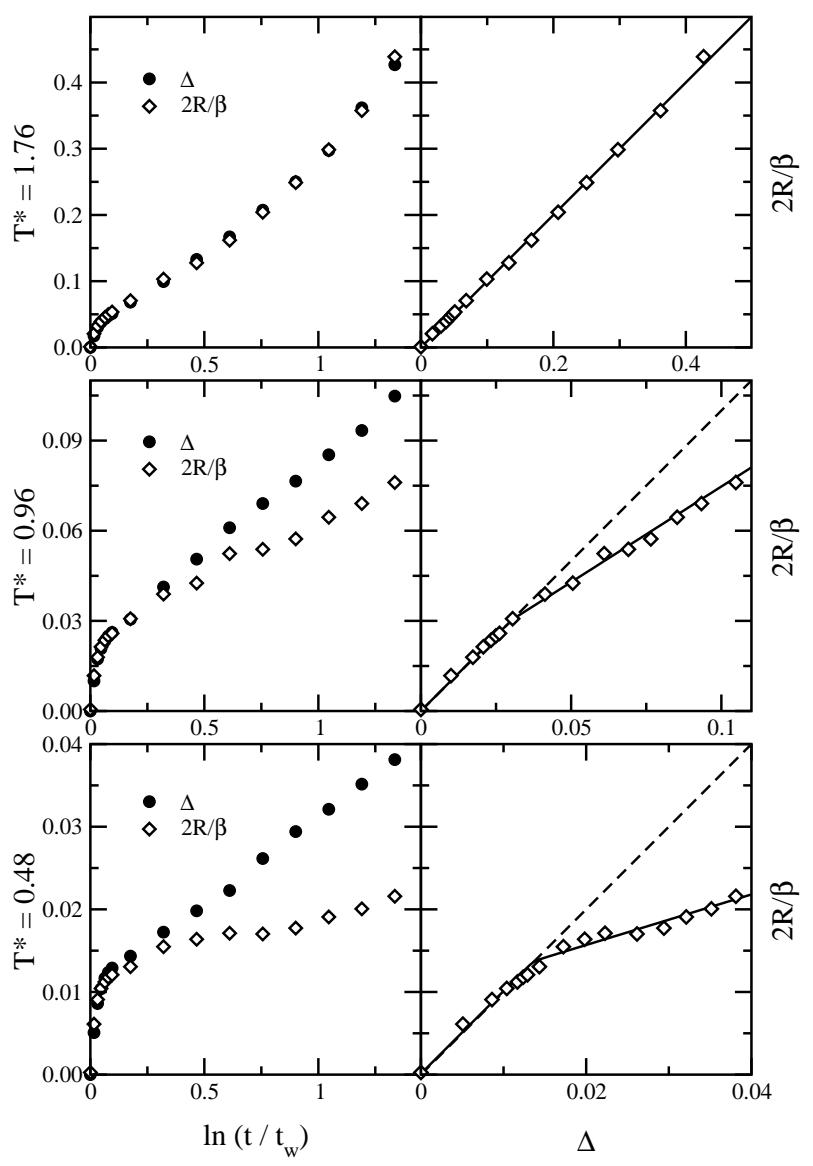

Figure 17. Mean square displacement $\Delta$ and integrated response function $2 R / \beta$ in reduced units at $\rho_{2}^{*}=1.24$ for three temperatures $T^{*}=0.48,0.96,1.76$. The left side shows the log time dependence of the two quantities. In the right side the response function vs $\Delta$. Dashed lines indicate equilibrium FDT, while full lines fit the off-equilibrium aging region. From 227 .

[71, 73, see also the discussion after (90). Inserting into (242) the value of $E_{\mathrm{IS}}$ as function of time one finally gets the function $T_{\text {eff }}(t)$. The definition of $T_{\text {eff }}$ is shown graphically in figure 18 238.

The two-timescales scenario is rather well confirmed by numerical results, as shown in the FD plots of figure 19 238, where the FD plot for the binary mixture Lennard-Jones system is reported. The full lines are the prediction from (242), the agreement is rather good.

\subsubsection{Finite-size mean-field glasses 65, 66, 67, 36].}

We have seen in the previous sections that the essential features of MCT for glass-forming systems are also common to some fully connected spin-glass models, called mean-field $p$-spin glasses with $p>2$. In the thermodynamic limit, the hightemperature paramagnetic phase is described by the schematic mode MCT for supercooled liquids. At the critical temperature $T_{\mathrm{c}}$ an ergodic to non-ergodic transition 


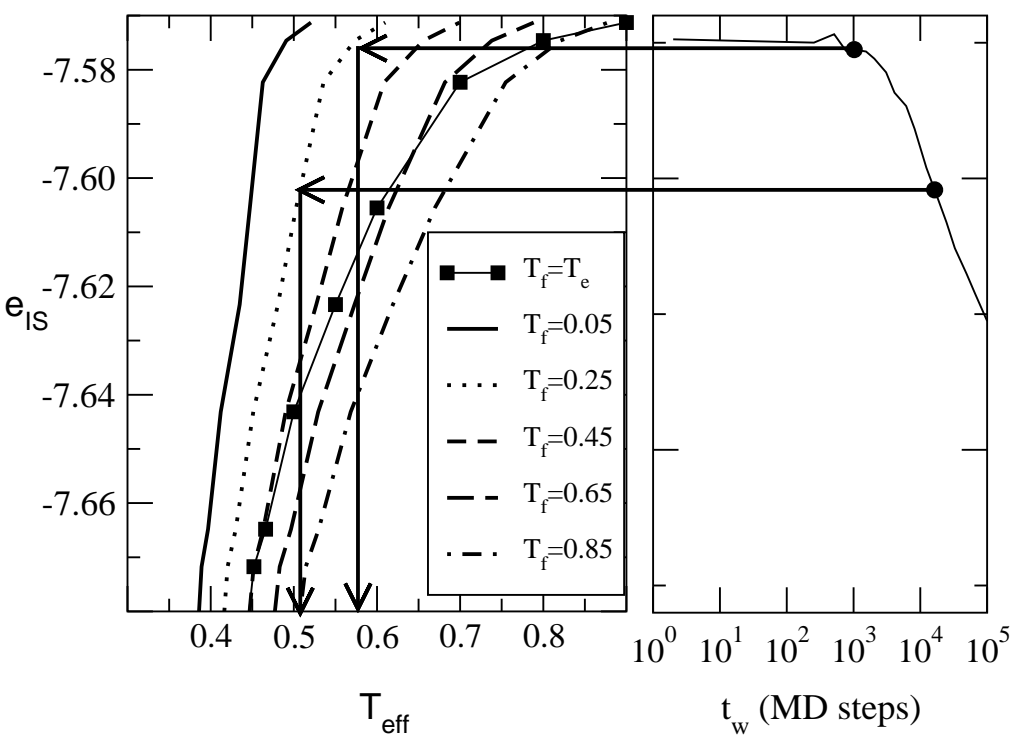

Figure 18. Left: Solutions of 242) for several values of of the final quench temperature $T_{\mathrm{f}}$ for the BMLJ system. Right: $e_{\mathrm{IS}}$ as a function of time, following the temperature quench. The arrows show graphically the procedure which connects the $e_{\mathrm{IS}}(t)$ value to $T_{\text {eff }}$ value, once $T_{\mathrm{f}}$ is known. [Data courtesy of F. Sciortino and P. Tartaglia, see also Ref. [238]]. From [36].

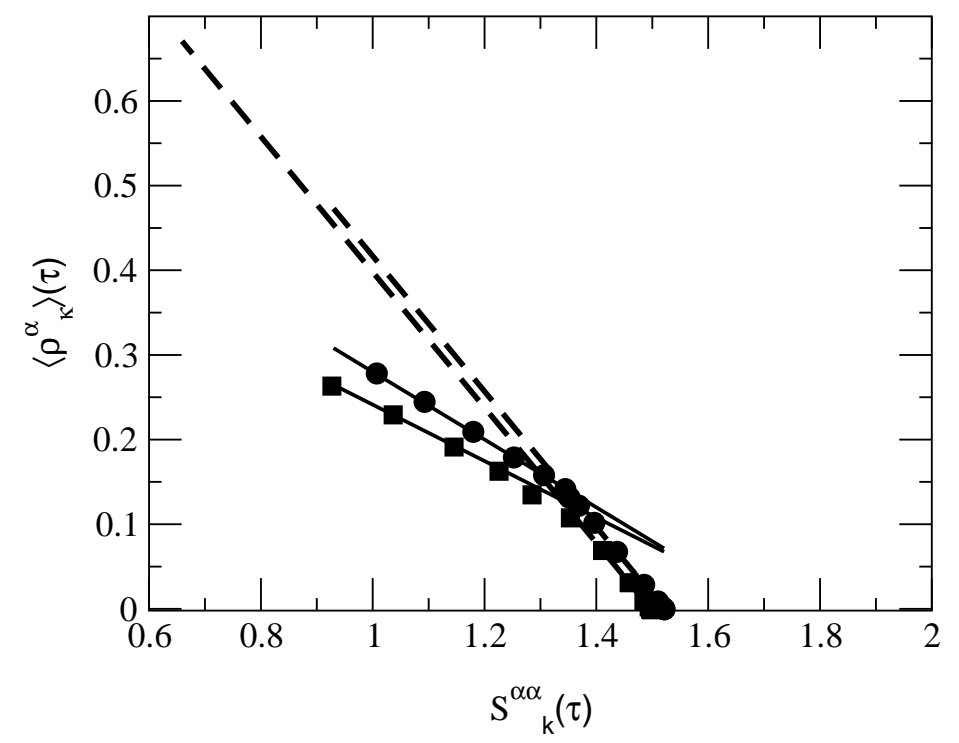

Figure 19. Response $\left\langle\rho_{\mathbf{k}}^{\alpha}(\tau)\right\rangle$ versus the dynamical structure factor $S_{\mathbf{k}}^{\alpha \alpha}(t) \equiv$ $\left\langle\rho_{\mathbf{k}}^{\alpha}(t) \rho_{\mathbf{k}}^{\alpha *}(0)\right\rangle$, where $\rho_{\mathbf{k}}^{\alpha}$ is the Fourier transform component of the density of $\alpha=$ $A, B$ particles at wave-vector $\mathbf{k}$, for the binary mixture Lennard-Jones particles system for a quench from initial temperature $T_{\mathrm{i}}=0.8$ to final temperature $T_{\mathrm{f}}=0.25$ and two waiting times $t_{w}=1024$ (square) and $t_{w}=16384$ (circle). Dashed lines have slope $T_{\mathrm{f}}^{-1}$ while thick lines have slope $T_{\mathrm{eff}}^{-1}$. [Data courtesy of F. Sciortino and P. Tartaglia, see also Ref. [238]]. From [36] 
takes place. In mean-field models the relaxation time diverges at $T_{\mathrm{c}}$ as barriers separating different ergodic components become proportional to the system size, thereby diverging in the thermodynamic limit. In real systems, or glass models just described, the barriers are of finite height and the transition to a glassy state appears at the glass transition temperature $T_{\mathrm{g}}<T_{\mathrm{c}}$, where the typical activation time over barriers is of the same order as the observation time.

Despite these differences mean-field models, having the clear advantage of being analytically tractable, are a very useful tool to study the phase space structure of glassy systems, especially between the dynamical temperature $T_{\mathrm{c}}$ and the static temperature $T_{\mathrm{RSB}}$ (Kauzmann temperature $T_{\mathrm{K}}$ in glass language). The main drawback is that, since activated processes are not captured by mean-field models, the picture that emerges is not complete. To go beyond mean-field it is necessary to include activated processes, a very difficult task since it implies the knowledge of the excitations involved in the dynamics. A simple approach is to include finite-size effects in the dynamics of an infinite mean-field system just extending the analysis to finite-size mean-field. This approach has been suggested by Nieuwenhuizen 49] and is somehow reminiscent of the dynamical approach of Sompolinsky [141], see also section 6.2.2 We stress that the assumption that finite-size mean-field models capture the physics of glasses beyond MCT is not trivial. In fact, activated process in finite-size mean-field models could be different from those of supercooled liquids, making the behavior different. This, for example, seems to be the case of the Potts Glass model, where recent studies on a finite-size version indicate some differences with the fragile-glass scenario [239, 240].

A spin-glass model in the $p$-spin universality class, that displays a fragile-glass behavior, is the Random Orthogonal Model (ROM) [241, 74]. The model is defined by the Hamiltonian $\dagger$

$$
\mathcal{H}=-2 \sum_{i j} J_{i j} \sigma_{i} \sigma_{j}
$$

where $\sigma_{i}$ are $N$ Ising spin variables $(\sigma= \pm 1)$ and $J_{i j}$ is a random $N \times N$ symmetric orthogonal matrix with zero diagonal elements. We note that at difference with previously discussed spin-models, the condition of orthogonality leads to a strong correlation among the matrix-elements. In the limit $N \rightarrow \infty$ this model has the same thermodynamic properties as the $p$-spin model. The dynamical transition is at $T_{\mathrm{c}}=0.536$ with threshold energy per spin $e_{\mathrm{th}}=-1.87$. A static transition occurs at $T_{\mathrm{RSB}}=0.256$ and the critical energy per spin is $e_{1 \mathrm{rsb}}=-1.936$ where the complexity vanishes 241, 74]. The analysis in the mean-field limit gives a rather clear "geometrical" interpretation of the two transitions. The phase space is composed by an exponentially large (in $N$ ) number of components, separated by infinitely large (for $N \rightarrow \infty)$ barriers. Each component is labeled by the energy density $e$ of its minimum and the largest allowable value of $e$ is $e_{\mathrm{th}}$ 76, 242]. Components with $e$ equal to $e_{\mathrm{th}}$ have the largest (exponentially with $N$ ) statistical weight and become dominant, in thermodynamic sense, at the dynamical transition $T=T_{\mathrm{c}}$. Since components with $e$ smaller than $e_{1 r s b}$ have negligible statistical weight [73, 74, the static transition is ruled by components with $e=e_{1 r s b}$, i.e., the lowest accessible ones [42, 73].

For finite $N$ the scenario is different since not only basins with $e<e_{\mathrm{th}}$ acquire statistical weight, but basins with $e>e_{\text {th }}$ with few negative directions [76] may

$\dagger$ The factor 2 in 243 is set only for convenience to match the values of all relevant temperatures with those reported in the original paper [241]. The Hamiltonian studied in 74 differs in a factor 4 from the present definition. To compare the results discussed here with those in 74 temperatures and energies must be properly scaled by a factor 4 . 
become stable, simply because for finite $N$ there are not enough degrees of freedom to hit them. The ROM for finite $N$ has been largely studied during the last years 65, 66, 67, 36, 243 and its behavior has been compared with that of supercooled liquids finding a remarkable agreement.

In the left panel of figure 20 it is shown the average energy-minima of basins (IS) as function of temperature for the ROM with $N=300$ obtained from a Monte Carlo simulation. It can be shown 60, 61 that if the density of states $\Omega(E)$ is Gaussian and if the basins have approximately the same shape or are, to a good degree, harmonic, then the IS energy density $e_{\mathrm{IS}} \propto 1 / T$. The data in the figure can be well fitted by

$$
e_{\mathrm{IS}}=e_{\infty}+e_{1} T^{-1}+O\left(T^{-2}\right)
$$

indicating that for a relatively large energy range the basins are roughly of the same shape. This means that the contribution $f_{v}\left(T, e_{\mathrm{IS}}\right)$ to the free energy density of the system is of the form $f_{v}\left(T, e_{\mathrm{IS}}\right)=e_{\mathrm{IS}}+\delta f_{v}(T)$, with the second term independent of $e_{\mathrm{IS}}$, i.e., of the component 65]. This in turn implies that the effective temperature for the ROM is completely determined once the complexity (density) $s_{c}(e)$ is known [66]. Indeed from (90, 242) we have

$$
\frac{1}{T_{\text {eff }}\left(e_{\mathrm{IS}}\right)}=\frac{\partial s_{c}\left(e_{\mathrm{IS}}\right)}{\partial e_{\mathrm{IS}}}
$$

Furthermore, in an aging experiment $T_{\text {eff }}$ depends on time only through $e_{\mathrm{IS}}(t)$. For each time $t$ the effective temperature $T_{\text {eff }}$ can be obtained graphically as shown in figure 20 The left panel in that figure shows the average $e_{\mathrm{IS}}$ energy as a function of time in a typical aging experiment. We note the two-regimes decay also observed in supercooled liquids 244. The two regimes are associated with different relaxation processes. In the first part the system has enough energy and relaxation is mainly due to path search out of basins through saddles of energy lower than $T$, where $T$ is the temperature after the quench. This part depends only on the temperature of the equilibrium state from which the system has been quenched. This process stops when all barrier heights become of $O(T)$ and relaxation slows down since it must proceed via activated inter component processes. In figure 21] it is shown the response versus correlation plot for the ROM. Correlations and responses were computed by projecting over the IS, the corresponding FDT also holds in equilibrium as discussed in section 3.3 The figure clearly show the two-timescales scenario with $X=1$ at short times and $X=T / T_{\text {eff }}<1$ at later times, with $T_{\text {eff }}$ in very good agreement with the value predicted by (242). Also notorious is the fact that the effective temperature shifts with time as expected.

\subsection{Spin glasses and other random systems}

As we have explained in section [6] spin glasses represent the most important motif of many results regarding FDT violations. Particularly, numerical simulations have been the most widespread tools to investigate many aspects of the equilibrium behavior of spin glasses that cannot be tackled by analytic means (for a review see [245]). It is usually said that the advantage that numerical simulations offer in the study of nonequilibrium properties, as compared to equilibrium ones, relies on the fact that systems do not need to be equilibrated. However this observation is naive and deceitful as many dynamical aspects cannot be observed in the range of accessible time-scales. Indeed, it is widely believed that many dynamical results in spin glasses are suspect because the asymptotic dynamical regime, defined as that regime where the dynamic correlation 


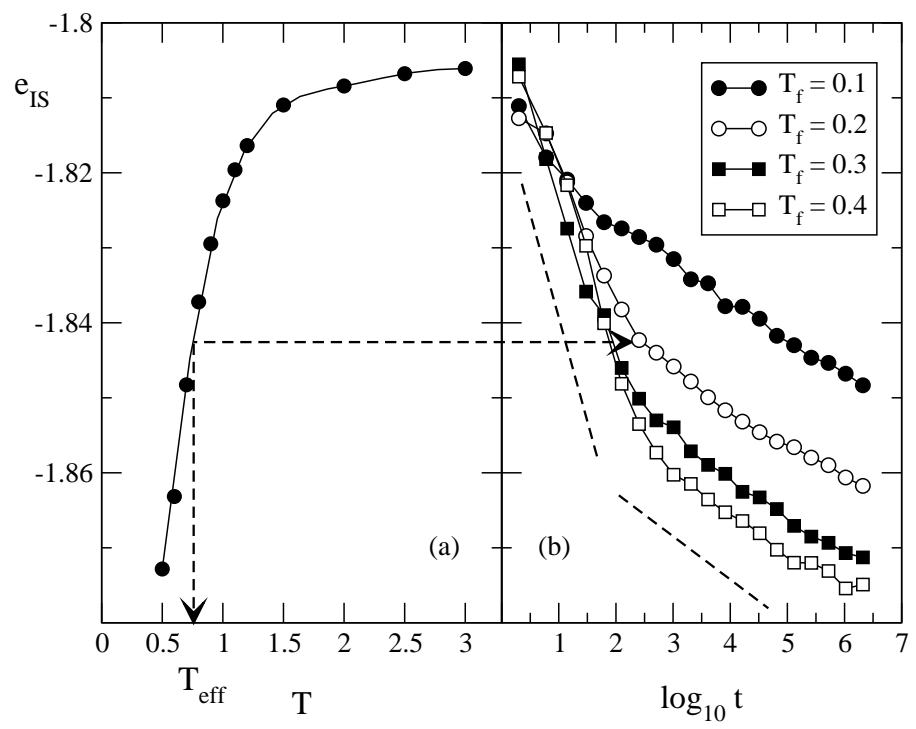

Figure 20. Panel (a): Equilibrium average $e_{\mathrm{IS}}$ a function of temperature. The arrows indicate the construction of the effective temperature $T_{\text {eff }}\left(e_{\text {IS }}\right)$. Panel (b): Average inherent structure energy for the ROM as function of time for initial equilibrium temperatures $T_{\mathrm{i}}=3.0$ and final quench temperatures $T_{\mathrm{f}}=0.1,0.2$, 0.3 and 0.4 . The average is over 300 initial configurations. The system size is $N=300$. The lines denotes the two regimes. [see also Ref. 66]] From [36.

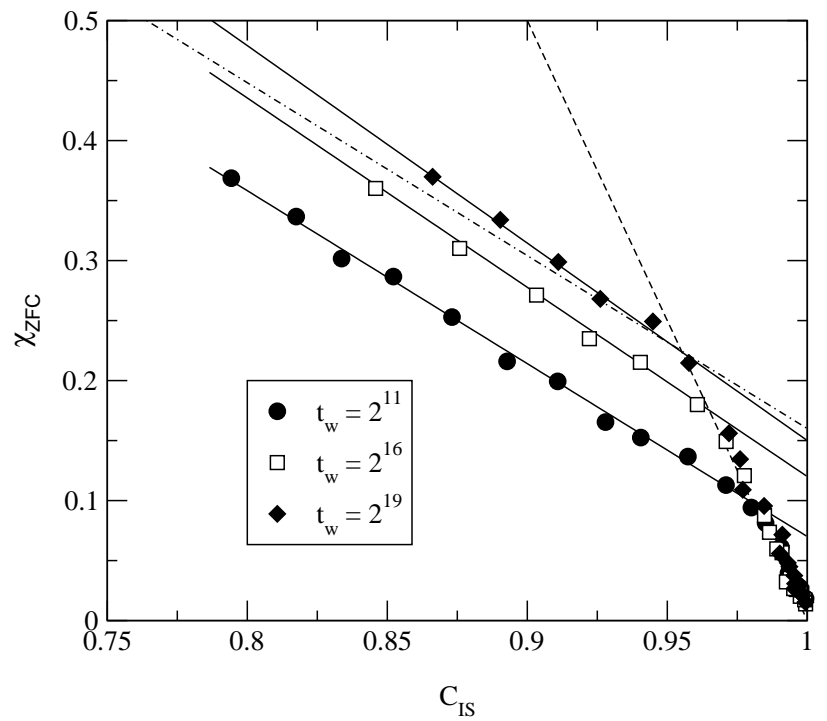

Figure 21. Integrated response function as a function of IS correlation function, i.e, the correlation between different IS configurations, for the ROM. The dash line has slope $T_{\mathrm{f}}^{-1}=5.0$, where $T_{\mathrm{f}}$ is the final quench temperature, while the full lines are the prediction 245): $T_{\text {eff }}\left(2^{11}\right) \simeq 0.694, T_{\text {eff }}\left(2^{16}\right) \simeq 0.634$ and $T_{\text {eff }}\left(2^{19}\right) \simeq 0.608$. The dot-dashed line is $T_{\text {eff }}$ for $t_{\mathrm{w}}=2^{11}$ drawn for comparison. [see also Ref. [66]. From [36]. 
length $\xi$ is many lattice spacings, is usually not reached. Establishing whether the range of simulated time-scales reaches the asymptotic long-time regime is at the heart of a present controversy in the field. Indeed, not by chance, this controversy is quite reminiscent of another parallel ongoing discussion concerning the magnitude of finite-size effects in the equilibrium properties. Stochastic stability arguments linking non-equilibrium with static properties [160, 161] confirm that any strong finite-size corrections to the equilibrium properties should manifest as strong finite time-scales corrections in dynamical experiments. Unfortunately, a precise theory that quantifies (even in an approximate way) these corrections is presently unknown. We will not deal here with the difficult issue of ascertaining in which cases simulations do reach the asymptotic time regime, but present the evidence on FDT violations for the accessible simulated time-scales.

7.2.1. Spin glasses We begin our tour by reporting the first numerical evidence of FDT violations in 3d EA spin glasses [246] and their representation in the form of FD plots [247]. In [246] it was shown that deviations from the equilibrium FDT appear at timescales comparable or larger than the age of the system. In figure 22 we show TRM measurements by Franz and Rieger [247] on the 3d EA model. In those measurements the systems starts from a random initial configuration and a magnetic field $h$ is applied for time $t_{w}$. The field is cut off at $t_{w}$ and the subsequent decay of the $M_{\mathrm{TRM}}\left(t, t_{w}\right)$ recorded. In the linear response regime this experiment is equivalent 248 to a ZFC setup where the field is initially zero and switched on at $t_{w}$, the resulting $M_{\mathrm{ZFC}}\left(t, t_{w}\right)$ being given by $M_{\mathrm{TRM}}\left(t, t_{w}\right)+M_{\mathrm{ZFC}}\left(t, t_{w}\right)=M_{F C}$ for $t$ large enough (see (99)) where $M_{F C}$ is the equilibrium magnetization. Most of the numerical simulations use the ZFC procedure. The most extensive simulations and the most clarifying FD plots, as described in section 4.3] have been done in $d=3$ and $d=4$ [249, 250, 251, 252, 253. In those papers, the authors consider the IRF associated to the global and the spin-spin autocorrelation function as described in section 4.3 The system is quenched at low temperatures for a time $t_{w}$ and a small magnetic field is subsequently applied and the $M(t)$ measured. Typical FD plots in the 3d Edwards-Anderson (EA) model using this construction are shown in figure 23. There we show $S(C)$ at two different magnetic field intensities as well as two different waiting times. FD plots reveal that a constant slope $X(C)$ for $C<q_{E A}$ is a good approximation to the data (although more accurate data in $4 \mathrm{~d}$ hint at the existence of a curvature in $S(C)$ 251, 250]). This constancy of the slope $X(C)$ (i.e. the linearity of $S(C)$ in the region where FDT is violated) can be also interpreted as complementary evidence of the accuracy of the $t / t_{w}$ scaling in the correlation function [254]. Stochastic stability arguments [160] 161] state that the dynamical $X(C)$ is related to the static function $x(q)$ by the relation $P(q)=x^{\prime}(q)$ or,

$$
x(q)=\int_{0}^{q} d q^{\prime} P\left(q^{\prime}\right)
$$

where $P(q)$ is the probability distribution of overlaps between replicas of the same system. This identity offers a way to obtain $S(C)$ from equilibrium data. The derivative of the relation (101) respect to $C$ yields $P(C)=-d^{2} S(C) / d C^{2}$. Inverting this identity and inserting an estimate of $P(C)$ as obtained from equilibrium simulations allows an alternative way to compute $S(C)$. The applicability of this method is shown in figure 23. A quantitative evidence of the mean-field character of the FDT violations has been also reported by checking the accuracy of the Parisi-Toulouse approximation in spin glasses (this approximation states that the order-parameter 

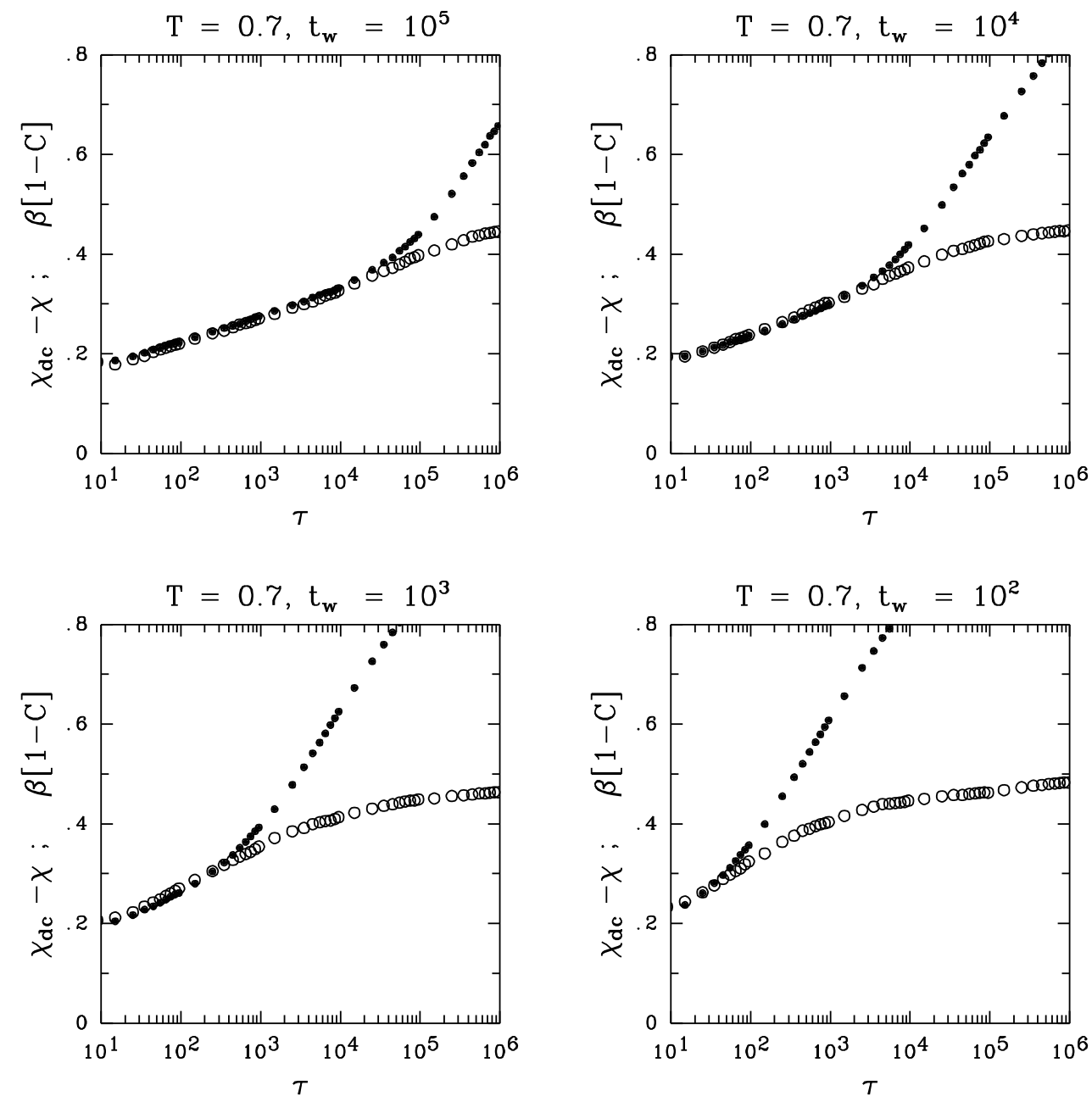

Figure 22. Zero-field cooled $\operatorname{IRF} \chi_{\mathrm{ZFC}}\left(t, t_{w}\right)=M_{\mathrm{ZFC}}\left(t, t_{w}\right) / h=\chi_{d c}-$ $\chi_{F C}\left(t, t_{w}\right)$ (empty circles) in the $3 \mathrm{~d}$ EA model plotted as function of the time $t \equiv \tau$ for different values of $t_{w}$ at $T=0.7$. For times $t \equiv \tau>t_{w}$ deviations from the FD relation $\chi_{\mathrm{ZFC}}\left(t, t_{w}\right)=\beta\left(1-C\left(t, t_{w}\right)\right)$ (filled circles) are noticeable. From [247.

function $q(x)$ is a function of the argument $x / T$, see 255, 256 for an exposition). Within this approximation (which works pretty well in MF spin glasses) it can be shown 249, 253 that $\chi\left(t, t_{w}\right) \equiv \chi(C)=\beta S(C)$ is independent of temperature in the region $C<q_{E A}$ where FDT is violated. In the SK model it can be proved that this function $\chi(C) \simeq \sqrt{(1-C)}$. In general, for any short-range system one can assume the following behavior: a) $\chi(C)=\beta(1-C)$ for $C>q_{E A}$ when FDT holds and b) $\chi(C)=A(1-C)^{B}$ for $C<q_{E A}$. Multiplying $\chi(C)$ by $T^{1-\phi}$ with $\phi=1 /(1-B)$ one finds that the resulting quantity is a sole function of the argument $(1-C) T^{-\phi}$ : $T^{1-\phi} \chi(C)=\hat{\chi}\left((1-C) T^{-\phi}\right)$ thereby showing that data for different waiting times and temperatures should collapse on a single master curve. Bot in $d=3,4$ a best collapse is obtained taking $B=0.41[249,253]$ which is quite close to the mean-field result 


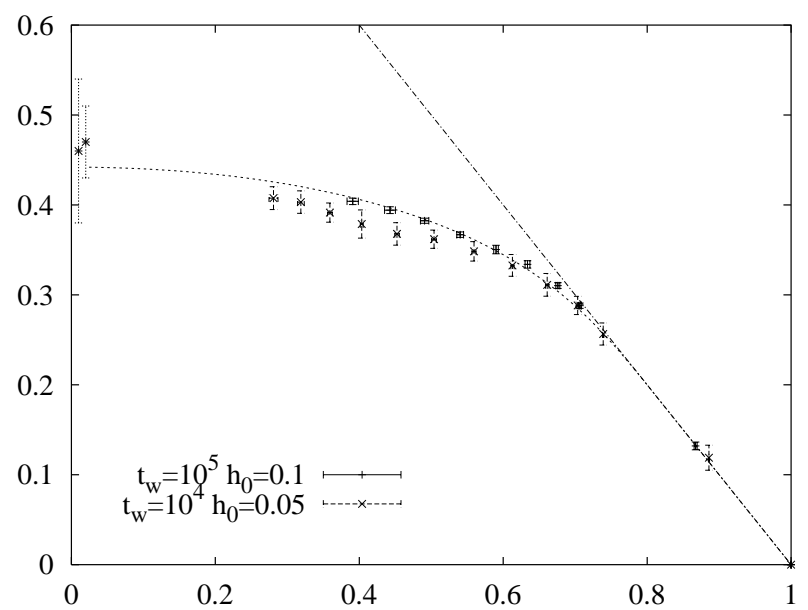

Figure 23. $S(C)$ versus $C$ in the $3 \mathrm{~d}$ EA model at $T=0.7<T_{\mathrm{c}} \simeq 1$ for $L=64$. The continuous line is the prediction obtained from equilibrium data for $L=16$ (averaged over 900 samples) as explained in the text. The straight line is the FDT prediction. From [249].

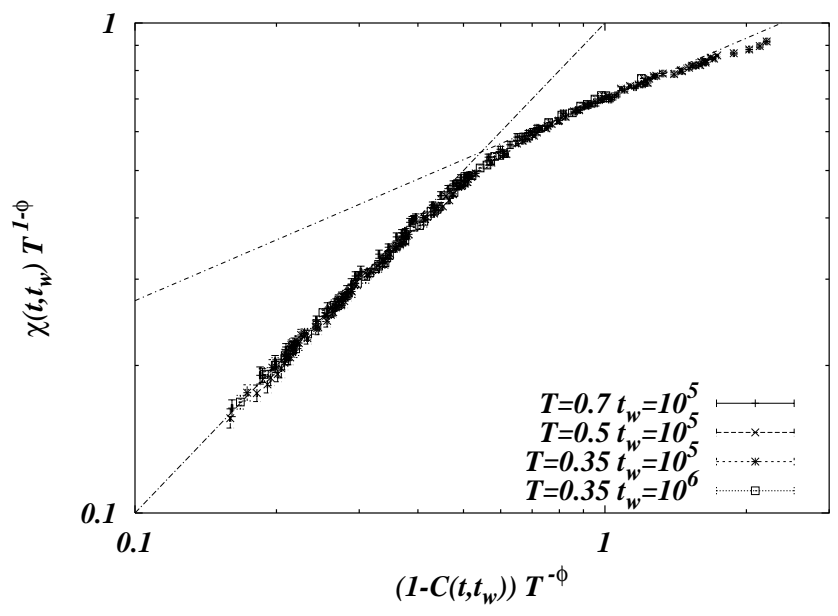

Figure 24. Scaling plot as described in the text for a cubic lattice, $d=3$ with $L=64$ showing the existence of two dynamical regimes described by two different scalings for the scaling function $\hat{\chi}(x)$ where $x=(1-C) T^{-\phi}$. In the FDT regime $\hat{\chi}(x) \sim x$ while for $x>1, \hat{\chi}(x) \sim x^{B}$ with $B \simeq 0.41$. From [253.

$B=1 / 2$, see figure 24

Apart form the EA model many other results have been obtained studying shortrange versions of the disordered p-spin model. Two different models have been considered. On the one hand there is the so called disordered plaquette model 257. where spins occupy the vertexes of a finite-dimensional lattice and the interaction occurs between $p$ spins belonging to a given plaquette. The Hamiltonian of this model 


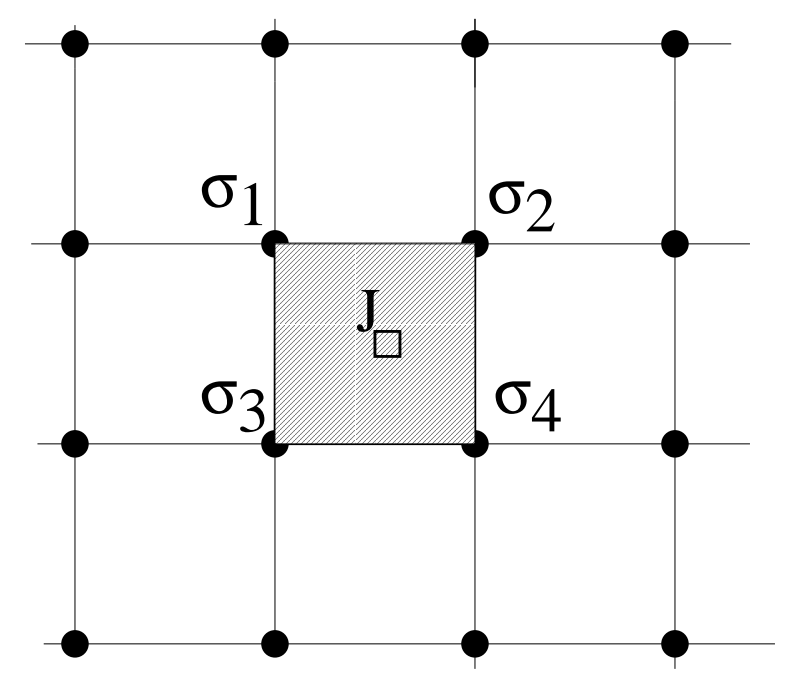

Figure 25. Schematic figure of the disordered plaquette model in $d=2$.

reads,

$$
\mathcal{H}=-\sum_{\square} J_{\square} \prod_{i \in \square} \sigma_{i}
$$

where ' $\square$ ' denotes a plaquette (not necessarily a square plaquette) that connects different spins. As usual, $J_{\square}$ are quenched variables of zero mean and finite variance and $\sigma_{i}= \pm 1$ denote Ising variables. The simplest case, the one considered in [257, is a disordered version of the $p=4$ model and consists of a regular lattice of side $L$ and dimension $D$ where spins occupy the vertexes and plaquettes correspond to the different faces of the lattice. Each face contains four spins and each spin belongs to $4\left(\begin{array}{l}D \\ 2\end{array}\right)$ plaquettes. A schematic picture of the lattice in $d=2$ is shown in figure 25 The study of the static and dynamics properties of this model revealed that, although there was no compelling evidence in favour of a finite $T$ spin-glass transition, the relaxation time shows superactivation effects and stretching of correlation functions characteristic of fragile glasses. The relaxation time can be fitted both to a VTF law with $T_{0}=0.65$ or to an exponential inverse temperature squared law with $T_{0}=0$. The study of the equilibrium properties confirmed both possible scenarios $\left(T_{\mathrm{c}} \sim T_{0}=0.65\right.$ or $\left.T_{c} \simeq 0\right)$ but show that, whatever scenario holds, the relaxation time $\tau$ and the equilibrium correlation length $\xi$ are linked by the relation, $\tau=A \exp (B \xi / T)$ supporting a scenario of cooperative dynamics. Also, the trap-like character of the dynamics was confirmed by studying the overlap among to identical replicas at $t_{w}$ but evolving with different noises, $Q\left(t_{w}, t_{w}+t\right)$. This quantity should coincide with $C\left(t_{w}, t_{w}+2 t\right)$ if jumps among configurations are uncorrelated or entropically driven. Numerical results are compatible with this prediction. Accordingly, the FD plot (figure 26) measured at $T=0.7$ (just above the suspected finite $T_{\mathrm{c}}$ ) showed strong deviations from the equilibrium line $\chi=\beta(1-C)$ with a FDR $x \sim 0.4$, and in agreement with the one-step pattern characteristic of structural glasses (see section 7.1).

On the other hand there is another short-range version of the $p$-spin model 258, 259, 152, 260, 261] where $M$ spins $\left(s_{i}^{1}, . ., s_{i}^{M}\right)$ occupy the different sites $i$ of a cubic lattice. For each two adjacent sites $i$ and $j$ one considers all possible groupings 


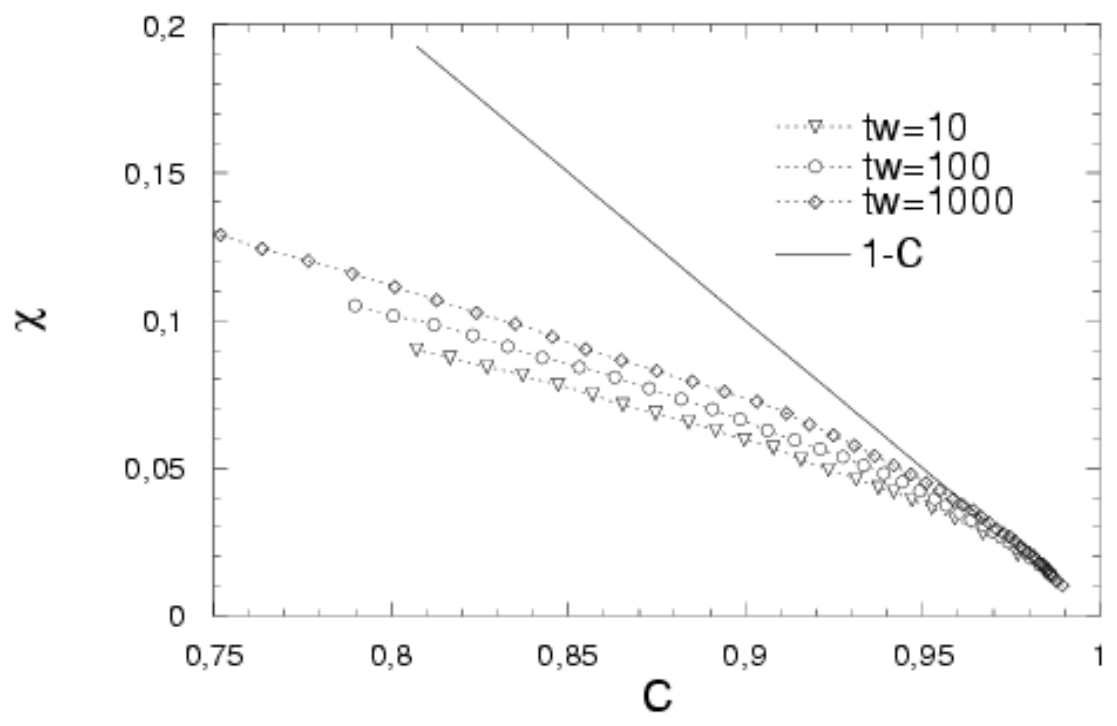

Figure 26. FD plot for the disordered plaquette model (with $p=4$ ) in a cubic lattice of lattice size $L=20$ at $T=0.7$ and three different waiting time values. From [257].

of different $p$ spins that can be formed by taking $k$ spins from site $i$ and $p-k$ spins from site $j$. In an obvious abuse of notation we can write,

$$
\mathcal{H}=-\sum_{(i, j)} \sum_{g \in(i, j)} J_{g} \prod_{k \in g} \sigma_{k}
$$

where the sum runs over all possible nearest neighbours $(i, j)$ and all different groups of $p$ spins as described above. Again, the $J^{\prime} s$ are quenched variables with zero mean and finite variance. Two cases have been considered: $M=2, p=3$ [258, 261] and $M=3,4, p=4$ [259] 260] (for $M=2, p=4$ the model reduces to the standard EA model 259]). In the first case the model is not time-reversal invariant while it is in the second case. FD violations have been measured in this last case [260]. Finite-size scaling studies of the model shows some evidence of a second order phase transition at $T_{\mathrm{c}}=2.6$ characterized by a divergent spin-glass susceptibility. This result is confirmed by a study of the FDT violations in this model that show the existence of a non-trivial $X(C)$ characteristic of a full RSB scenario. As for the EA model, the $X(C)$ appears to be linked to the static $P(q)$ via the relation $P(q)=X^{\prime}(q)$, see figure 27. The main message conveyed by most of these results is that FDT violations are qualitatively and quantitatively well described in the framework of MF theories of spin glasses. However, the implications of these similarities must not be taken too far. In particular, the already old but recurrent issue about the validity of the many state picture in finite-dimensional spin glasses cannot be answered from such a point of view. As discussed previously, the precise link between statics and dynamics proposed by stochastic stability arguments confirms that the dynamic $X(C)$ is related to the static $x(q)$ after introducing a coherence length $l\left(t_{w}\right)$ (related to the spin-glass correlation length $\xi\left(t_{w}\right)$ obtained from the two-point replica correlation function) which depends 


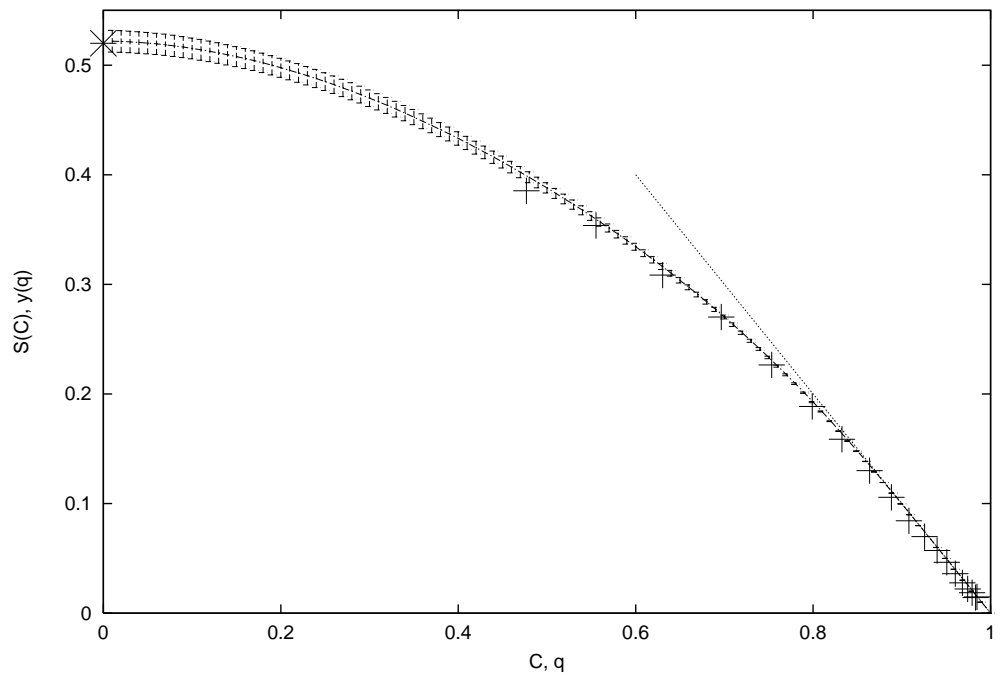

Figure 27. FD plot for the short-range version of the $p$-spin model (with $p=4, M=3$ ) in a cubic lattice of lattice size $L=16$ at $T=2.0=0.77 T_{\mathrm{c}}$ and $t_{w}=2^{1} 7$. The continuous line with error bars is the shape of $X(C)$ derived by numerical integration of the relation $P(q)=x^{\prime}(q)$ and measuring the equilibrium $P(q)$ for $L=5$, the dotted line is the equilibrium line $S(C)=1-C$ and the isolated cross at $C=0$ correspond to the FC magnetization. From [260].

on the waiting time $t_{w}$ according to the relation $x\left(q, l\left(t_{w}\right)\right)=X\left(C\left(t, t_{w}\right)\right)$. A numerical study of FDT violations in $d=2$ [262] shows that such an assumption is indeed true and $l\left(t_{w}\right) \propto \xi\left(t_{w}\right)$. More important, the resulting FD plots are extremely similar to those found in $d=3,4$ and the scaling Ansatz for $S(C)$, as derived from the assumption that the low $T$ phase has many states, also works pretty well. However, as in $d=2$ there is no finite $T$ transition these results show that statements in favour of the validity (or not) of the mean-field picture in the accessible range of timescales (in off-equilibrium experiments) or sizes (in equilibrium measurements) are inconclusive. The stochastic stability property is well satisfied also in the XY model at not too low temperatures [220] where critical fluctuations dominate, the overall resulting behavior being quite similar to that of the $3 \mathrm{~d}$ EA model. From a completely different perspective, the overall presence of mean-field aspects in the analysis of offequilibrium data suggests that the many state picture is effectively valid and that only for experimentally inaccessible sizes or timescales (therefore irrelevant from a practical point of view) the true scenario (whatever mean-field or droplet) is recovered.

We finish this subsection by commenting some recent results 263 aiming to identify and quantify the low-energy fluctuations that locally result in deviations from the average QFDT as measured in the bulk. Local deviations from the bulk QFDT curve $\chi(C)$ are the equivalent of fluctuations from the average magnetization in a Heisenberg magnet, where transverse fluctuations correspond to low-energy spinwave excitations and the longitudinal fluctuations that modify the length of the magnetization vector being the massive ones. Numerical results in the $3 d$ EA model show that local correlations $C_{l}\left(t, t_{w}\right)$ and IRFs $\chi_{l}\left(t, t_{w}\right)$ measured over local boxes spread over the whole lattice generate a two-dimensional surface $\rho\left(C_{l}, \chi_{l}\right)$ with a prominent maximum centered around the bulk curve. The contour lines of this density 
map gently deform along the QFDT bulk curve $\chi(C)$ and deviations far away from that curve appear to be penalized. This study offers the possibility to understand the connections between the mean-field character of the FDT violations and the existence of deviations due to short scale cooperative processes in an eventual (but yet unclear) heterogeneous scenario.

7.2.2. Other random systems Apart from spin glasses other lattice models with quenched randomness have been considered in the literature aiming to elucidate whether off-equilibrium studies can tell something about the character of the lowtemperature phase. Many of the conclusions of these numerical studies need to be taken cautiously as no conclusive evidence in support of a given scenario or in refusal of other ones is ever reached.

Let us start the discussion with the ferromagnetic diluted and random-field Ising models (RFIMs). The 3d version of both models has been investigated in 250. Simulations in the low $T$ phase and in the Griffiths phase (i.e. the region of temperatures between the critical temperature of the pure system and the transition of the random system) show that FD plots in ferromagnetic diluted and RFIMs are very similar one each other but quite different to those measured in spin-glass systems (see the preceding section [7.2.1). The former ones are characteristic of a ferromagnetic phase with $X=0$ while the latter are described by a non-trivial function $X(C)$. These studies exclude the possibility of a spin-glass and Griffiths phase in both models described by a mean-field like RSB solutions.

Other finite-dimensional model with interesting behavior is the frustrated Ising lattice gas (FILG) 264 defined by the Hamiltonian,

$$
\beta \mathcal{H}=-J \sum_{\langle i, j\rangle}\left(\epsilon_{i j} \sigma_{i} \sigma_{j}-1\right) n_{i} n_{j}-\mu \sum_{i=1}^{V} n_{i}
$$

where the $\sigma_{i}= \pm 1$ are Ising spins and $n_{i}=0,1$ are occupancies which may take the value 1 or 0 depending whether site $i$ is occupied by a spin or not. The sum is over near-neighbours on a $d$-dimensional lattice. The $\epsilon_{i}$ are quenched random variables that may take the values \pm 1 and $\mu$ stands for a chemical potential. The average particle density $\rho=\frac{1}{V} \sum_{i} n_{i}$ is a monotonically increasing function of $\mu$. In the limit $J \rightarrow \infty$ the model converges to the site frustrated percolation problem 264] (a variant of the standard percolation problem where clusters are made out of sites connected by non frustrated links). This model has been simulated in 3d 265] 266 where different regimes have been singled out. The percolation transition occurs at a given value of the chemical potential $\mu_{p}$ and manifests in the onset of two different relaxational regimes (a fast exponential relaxation followed by a slow stretched decay). A second transition is observed at a higher value of $\mu, \mu_{d}>\mu_{p}$ where the relaxation time grows extremely fast and dynamics arrests. However, it is unclear whether the relaxation time diverges at $\mu_{d}$. Less clear is, in the case of the existence of a dynamical singularity at $\mu_{d}$, whether this is associated to a thermodynamic singularity. For the FILG (249) different correlation functions can be constructed depending on whether spin variables $\sigma$ or occupancies $n$ are considered. A study of FDT violations in this model leads to the following conclusions 266: 1) In the glassy regime $\mu>\mu_{d}$ dynamics is one-step like, i.e. a two-timescale scenario with two temperatures describes pretty well the relaxational behavior; 2) The effective temperature $T_{\text {eff }}$, as derived from the slope of the FD plots, is pretty independent on 


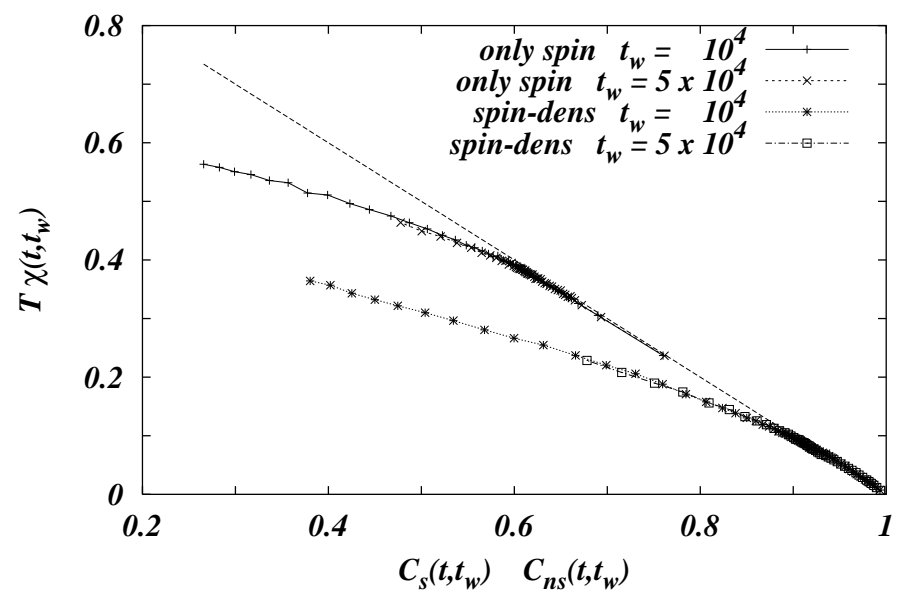

Figure 28. FD plot for the 3d FILG 249) with lattice size $L=30$ at $T=1, J=10, \mu \simeq 5.5$. Spin-density and spin autocorrelations (see the text) yield FD plots compatible with the same effective temperature. From [266].

the observable, whether this corresponds to spin variables $\left(C\left(t, t_{w}\right)=\left\langle\sigma(t) \sigma\left(t_{w}\right)\right\rangle\right)$ or mixed spin-occupancy variables $\left(C\left(t, t_{w}\right)=\left\langle\sigma(t) n(t) \sigma\left(t_{w}\right) n\left(t_{w}\right)\right\rangle\right)$ and 3$) T_{\text {eff }}$ is apparently independent on the waiting time. However, this last result has to be taken with caution as the range of waiting times considered in [266] may not be large enough $\ddagger$ to display such a small effect (compare for instance with the results described in section 7.1 figure 211). The observable independence of $T_{\text {eff }}$ is shown in figure 28 It should be noted that the above scenario is reminiscent of 1RSB behavior and occurs in finite $D$ rather than in the mean-field limit $D \rightarrow \infty$. Actually the mean-field version of the model does not have a 1RSB low temperature phase [267].

\subsection{Coarsening systems}

Although coarsening has been briefly sketched in section 6.6 here we present a more detailed account of results. Coarsening systems are the paradigm of systems which do not reach equilibrium. In such systems TTI does not hold, and all time-dependent correlation functions for large times are of the form $C(t, s)=C(L(t) / L(s))$ where $L(t) \propto t^{1 / z}$ is the typical size of the coarsening regions [268. The dynamic exponent $z$ is characteristic of the universality class of the system and its value depends on whether dynamics conserves or not the value of the order parameter. This functional form is similar to that found for the long-time correlation functions of glasses in the aging regime, and indeed a certain type of coarsening has been advocated as responsible for slow relaxation in glasses [269, 270, 271, 51]. The difference between the two systems only becomes manifest when one also considers the response functions associated to the correlation functions. Glasses, such as spin glasses or molecular glasses, are characterized by long term memory which results in a non-zero FDR $X$. On the other hand, for systems stochastically stable [160 161] the FDR $X(C)$ coincides with the static Parisi's function (246). For a ferromagnetic system $P(q)$ is trivial

$$
P(q)=\delta\left(q-m^{2}\right)
$$

$\ddagger$ In fact, the range of waiting times explored does not even cover one order of magnitude. 
where $m=m(T)$ is the magnetization a temperature $T$, and hence $X$ is 1 if $1>C>m^{2}$ and 0 if $C<m^{2}$. The argument can be easily extended to the case of few separate phases. Therefore we expect that in systems in which two (or few) phases separate, $X$ should vanish for long times, signaling the presence of weak long term memory.

The simplest model displaying domain-growth is a ferromagnetic Ising model on a square or cubic lattice of linear size $L$ with a single-spin-flip Glauber dynamics. When the system is quenched at time $t=0$ from a random configuration $(T=\infty)$ to a finite temperature $T$ below the critical temperature $T_{\mathrm{c}}$ domains of "up" and "down" spins start to form and grow. This is well reflected by the behavior of the two-times spin-spin correlation function

$$
C(t, s)=\frac{1}{N} \sum_{i=1}^{N}\left\langle\sigma_{i}(t) \sigma_{i}(s)\right\rangle
$$

which for times $t-s \ll s$ (assuming $s<t$ ) is TTI and rapidly decays from 1 to $m^{2}$, $m$ being the average magnetization at temperature $T$. Later, for more separate times $t-s \gg s$ the TTI is lost, the aging part of the correlation scaling like

$$
C_{\text {ag }}(t, s)=F\left[\frac{L(t)}{L(s)}\right]
$$

where $L(t)$ is the typical size of the domains at time $t$. The calculation of the linear response proceeds as usual, i.e., at a certain waiting time $t_{w}$ a small magnetic field $h_{i}$ is applied and the induced magnetization is computed. For disordered systems, such as spin glasses, the applied field can be either uniform or random. The advantage of an uniform field is that averaging over different realizations of the field is avoided. However, for systems without disorder, such as ferromagnetic systems, an uniform field would favor one of the phases making it growing faster. In this case a random field must be used and the correct quantity to measure is the staggered magnetization 272]:

$$
M(t, s)=\frac{1}{N} \sum_{i=1}^{N} \overline{\left\langle\sigma_{i}(t) h_{i}\right\rangle}
$$

where $h_{i}$ is the local (quenched) random field, and the over-bar denote overage over the field realizations.

In figure 29] we show the curves $\chi\left(t, t_{w}\right)=M\left(t, t_{w}\right) / h$ versus $C\left(t, t_{w}\right)$ obtained with a bimodal field distribution $h_{i}= \pm h$ [272]. The FDT region and the flattering of the curve are well evident. Two aspect of these curves are worth to be noted. First of all the value of the plateau reached by the magnetization decreases as $t_{w}$ increases. Moreover, for fixed $t_{w}$ the magnetization first grows in the non-aging part as $h(1-C) / T$, then saturates and eventually goes down again. Indeed, the comparison 273] with the equilibrium response function shows that the equilibrium value of the response lies rather below the plateau. The study of a soft spin version with Langevin dynamics [272, 273] leads to similar results.

There are two contribution to the staggered magnetization: one from the domain walls, the other from the domain bulks. The difference between the plateau and the equilibrium value of $M\left(t, t_{w}\right)$ can be attributed to the domain wall response. After a time $t_{w}$ the domains have reached a certain typical size, and the domain walls have a certain total length. The effect of the random field is to try to flip some spins. 

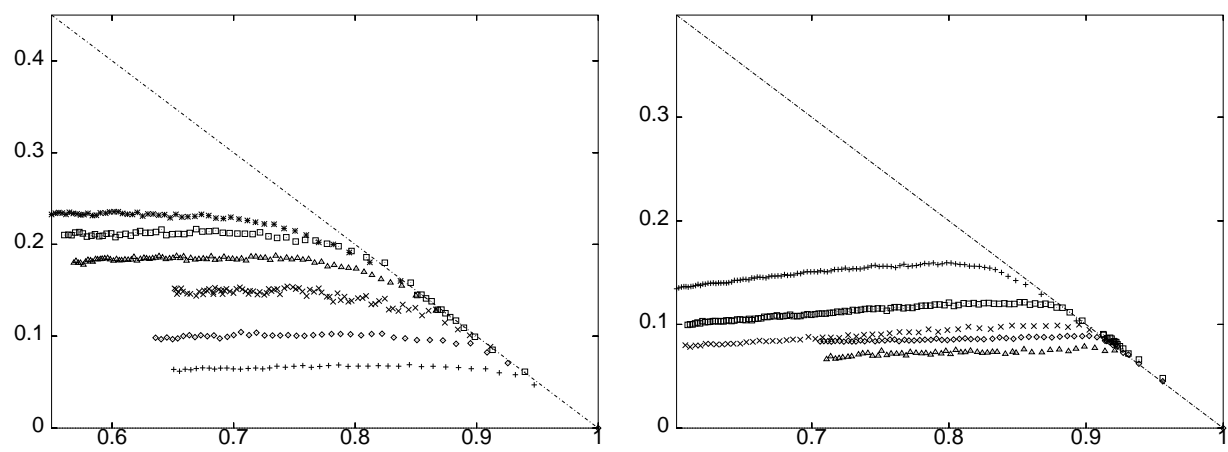

Figure 29. $T M\left(t, t_{w}\right) / h$ versus $C\left(t, t_{w}\right)$ domain growth. Left Panel: $2 \mathrm{~d}$ case with $T_{\mathrm{c}}=2.27$, at temperatures (from top to bottom) $T=1.7$ and $t_{w}=200,400,800,2000, T=1.3$ and $t_{w}=800, T=1$ and $t_{w}=800$. Right panel: $3 \mathrm{~d}$ case with $T=2.5\left(T_{\mathrm{c}} \approx 3.5\right), t_{w}=100,300,600,1000,1500$. The straight line is $M=1-C$ : we see that FDT holds at short times $t$, and the violation of FDT with $X=0$ at longer time separation. From 272

Clearly the flipping is easier at the domain walls where the spins are less constrained by their neighbors. As the time proceeds the domains grow increasing the bulks at the expense of the total domain wall length. Therefore the contribution from the interfaces decreases with time. On the other hand, the contribution of the bulk is almost independent of $t_{w}$ since the effect of a random field on "up" and "down" domains is the same on average. Therefore, after the initial quasi-equilibrium growth, the total staggered magnetization decreases as $t_{w}$ increases and, for a fixed value of $t_{w}$ it decreases as $t$ increases. Analytical results [273] show that the aging part of the IRF is of the form

$$
M_{\mathrm{ag}}(t, s) \sim A(s) F\left[\frac{L(t)}{L(s)}\right] \quad C_{\mathrm{ag}}(t, s) \sim F\left[\frac{L(t)}{L(s)}\right]
$$

where

$$
\begin{aligned}
A(t) & \sim \frac{1}{L(t)} \quad d>2 \\
& \sim \frac{\ln (L(t))}{L(t)} \quad d=2 .
\end{aligned}
$$

Because $X(s) \sim\left|\frac{\partial A(L(s))}{\partial L(s)}\right|$ the results (255) explain the slower decrease of the IRF for the two-dimensional case observed in figure 29. The numerical test of the scaling law (255) in $2 \mathrm{~d}$ is shown in figure 30.

This scenario leads to the conclusion that coarsening systems do not display a non-trivial $X(C)$. Recent results [217], however, indicate the possibility of non-trivial $X(C)$ also in these systems. The motion of the domain wall in the presence of an external random field follows from two competing processes: the tendency to reduce the interface curvature due to surface tension and the pinning of the domain wall in favorable positions introduced by the external field. This introduces a dependence on the space dimension since the curvature process, which dominates at large enough dimensions, weakens as the dimension decreases. When dimension reaches the lower critical dimension the curvature process disappears and the response of the system becomes non-trivial [217, 214] as it is indeed seen for the ferromagnetic Ising chain 


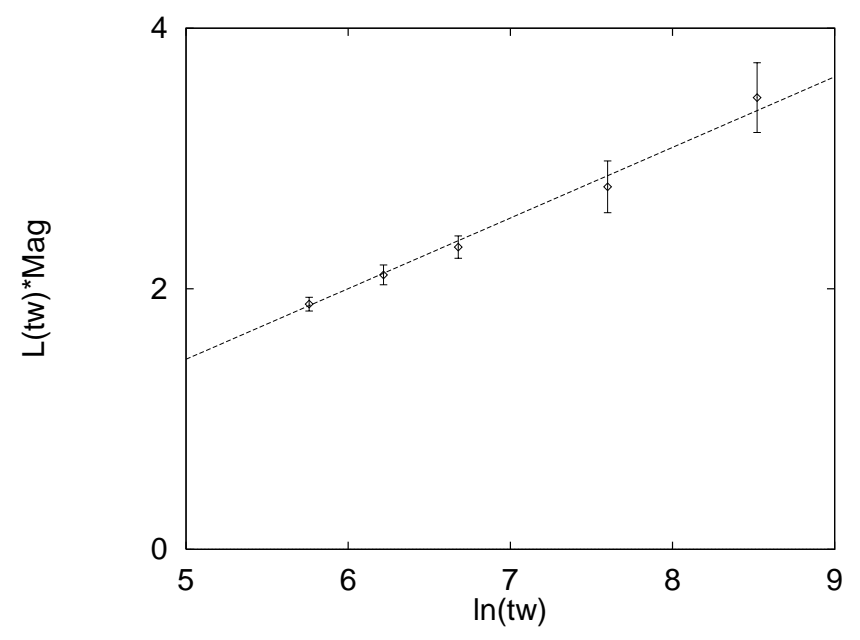

Figure 30. Test of the scaling (254) - 255) which predicts a linear dependence of $L(s) M_{a g}(t, s)$ w.r.t. $\ln (s)$ with $s=t_{w}$. The dashed line fits this dependence very well. From 273 .

$(d=1)[212,182 \S$.

The domain walls may give a large contribution to the response also at the early FDT part, but almost exclusively from their deformation on relatively short lengths. These fluctuations can be considered thermalized and hence do not spoil the $1 / T$ behavior but, on the contrary, contribute to make longer the $1 / T$ slope of the initial part in the FD plot. A generalization of the Ising model to include some frustration via long-range anti-ferromagnetic interactions has been studied in [274]. Models of this type have been proposed to study, among others, the avoided phase transition in supercooled liquids [275] and charge density waves in doped antiferromagnets [276] $\|$. They are described by the Hamiltonian

$$
\mathcal{H}=-\delta \sum_{\langle i, j\rangle} \sigma_{i} \sigma_{j}+\sum_{(i, j)} \frac{\sigma_{i} \sigma_{j}}{r_{i j}^{3}}
$$

where $\sigma_{i}$ are Ising spins, the first sum runs over all pairs of nearest-neighbor sites of the lattice, the second over all distinct pairs, and $r_{i j}$ is the distance between sites $i$ and $j$. The parameter $\delta$ represents the local/non-local exchange ratio. It is known 278] that for a two-dimensional square lattice the ground state of the model is anti-ferromagnetic for $\delta<0.85$. For $\delta>0.85$ the anti-ferromagnetic state becomes unstable with respect to formations of striped domains. The study of the non-equilibrium dynamics reveals a crossover from a logarithmic decay for $\delta<\delta_{c} \sim 2.7$ to an algebraic decay for $\delta>\delta_{c}$ 274]. However despite this richer scenario, the FD plot leads in both cases to a vanishing $X$, and hence to a coarsening scenario. Similar results are found in other coarsening-like systems such as the Migdal-Kadanoff spin glass [279] although in that model the definition of the dynamics appears rather tricky as most of the spins occupy the deepest hierarchical layer in the model.

$\S$ See also the discussion in section 6.6]. Similar effects are also observed in kinetically constrained models described in Sec. [7.5]

|| These models are of interest for the information storage in ultra-thin ferromagnetic films [277] 
We conclude this section on coarsening discussing a hard-sphere lattice gas model in the spherical approximation originally introduced by Lebowitz and Percus (LP) 280]. The interest in this model is twofold. On the one hand, its dynamical behavior can be solved analytically, hence allowing a detailed investigation of the non-equilibrium behavior. On the other hand, it is known that hard-sphere models have a fragile-glass behavior [90.

Lattice-gas models are defined on a lattice of finite dimensions. On each site there can be a density $\rho(\boldsymbol{x})$ of particles, which in the limit of hard-spheres can take only the values 0 (empty) or 1 (occupied). In the LP model this restriction is relaxed and $\rho$ takes any continuous value allowed by a spherical constraint:

$$
\sigma_{1} \equiv \sum_{\boldsymbol{x}} \rho(\boldsymbol{x})^{2}-\sum_{\boldsymbol{x}} \rho(\boldsymbol{x})=0
$$

There is the additional restriction that the density-density correlation function between nearest neighbors vanishes. This is added to mimic some kind of extended hard-core:

$$
\sigma_{2} \equiv \sum_{\boldsymbol{x}} \sum_{\boldsymbol{q}} \rho(\boldsymbol{x}) \rho(\boldsymbol{x}+\boldsymbol{q})=0
$$

where $\boldsymbol{q}$ are the vectors that join a lattice site $\boldsymbol{x}$ to its nearest neighbors. In [31] the following Langevin dynamics for an open system has been studied

$$
\frac{\partial}{\partial t} \rho\left(\boldsymbol{x}^{\prime}, t\right)=\mu-\frac{\partial}{\partial \rho\left(\boldsymbol{x}^{\prime}\right)} \mathcal{H}\left[\rho\left(\boldsymbol{x}^{\prime}\right), t\right]+\eta\left(\boldsymbol{x}^{\prime}, t\right)
$$

where $\mu$ is the chemical potential, $\eta$ the thermal noise, and $\mathcal{H}$ the Hamiltonian

$$
\mathcal{H}=\lambda_{0}(t) \sum_{\boldsymbol{x}}\left[\rho(\boldsymbol{x})^{2}-\rho(\boldsymbol{x})\right]+\lambda_{1}(t) \sum_{\boldsymbol{x}} \sum_{\boldsymbol{q}} \rho(\boldsymbol{x}) \rho(\boldsymbol{x}+\boldsymbol{q})
$$

Strictly speaking in this model there is no energy, but only entropy. The role of $\lambda_{0}(t)$ and $\lambda_{1}(t)$ in the Hamiltonian is to make the dynamics to fulfill the constraints (257) and (258) at all times. The non-equilibrium dynamics of this model shares a large number of features with that of the spherical SK model [281, 149, 150] where the dynamics is driven by the macroscopic condensation of the system onto the disordered ground state. A positive chemical potential would increase the local density, thus starting from an empty state the local density relaxes toward the equilibrium value. The relaxation can be divided into two regimes, the first one where the system is filled in a spatially uncorrelated way. The typical time for this process is order $t^{*} \sim O(1)$. It is only later that slow relaxation starts when the system is spatially correlated and needs to reorganize large regions in order to increase its density. The model has no built-in disorder, and the slowing down is a purely entropic, direct consequence of the decrease of the number of available configurations imposed by the the shortrange constraints. When the temperature is below the critical temperature $T_{\mathrm{c}}$ the two motions have well separated timescales and the two-times correlation function shows the usual two-steps form with a first part TTI and the second part scaling as $t / t_{w}$, see figure 2 (that plot corresponds to the $3 \mathrm{~d}$ model at $T=0.1$. Different waiting times from top to bottom are $\left.t_{w}=10000,1000,300,100,30,10,3,1\right)$ The study of the FDR reveals that there is no anomaly in the response function [31 and $X$ vanishes for values of $C$ below the plateau value of the correlation. The glassy scenario of this model corresponds to that of phase-ordering kinetics with non-conserved order parameter. Similar results have been reported in spherical models with long-range ferromagnetic interactions [282]. 


\subsection{Non-relaxational driven systems}

We have been underlining throughout this review that aging systems are characterized by a non-equilibrium behavior with lack of TTI and by the presence of FDT violations. The relevant parameter which controls the aging non-equilibrium state is the waiting time or time elapsed since the system was quenched. However, there is another way to generate a non-equilibrium state that can be characterized by a given timescale in the same fashion as the waiting time characterizes the aging state. For instance, adding a time-dependent perturbation of frequency $\omega$ to a time-independent Hamiltonian. In the regime where $\omega t_{w} \ll 1$ the perturbation oscillates slow enough to probe only slow processes occurring at time-scales $\tau \gg t_{w}$. While in the opposite regime $\omega t_{w} \gg 1$ the oscillatory perturbation probes fast relaxation processes occurring at timescales $\tau \ll t_{w}$. The line $\omega t_{w} \sim 1$ marks the onset of glassy behavior, the shape of this line depending also on the intensity of the perturbation.

Driven systems have advantages when compared to aging systems. One of the most important differences is that driven systems, in the stationary state, are TTI but FDT is still violated. Again the concept of an effective temperature can be introduced as a measure of these violations. However, as the non-equilibrium stationary state of driven systems can be described by the intensity of the driving force, they are more experimentally accessible than aging systems, where the waiting time appears as an external parameter difficult to control. For this reason, it has been advocated that experimental measurements of FDT violations and the effective temperature should be done in driven systems rather than aging systems.

There are many ways to put a system into a driven stationary state and these have been investigated in the literature for different types of models. Driven systems can be classified into two main groups:

- Sheared systems. In this case one considers systems where, in addition to conservative forces, other non-conservative forces (i.e. that cannot be derived from a potential function) act upon the system. In these systems the non-conservative forces maybe time-dependent or not. In both cases, the non-conservative forces do net work along a given closed dynamical path. This implies that energy power is continually supplied to the system by the driving force. The parameter which describes the stationary state is the intensity of the shearing or driving force which we will identify by the symbol $\epsilon$ or $\dot{\gamma}$. These systems include models that violate the action-reaction principle (such as models with asymmetric couplings) and sheared fluids. These systems are described in section 7.4.1

- Tapped systems. In this class, systems are driven to a non-equilibrium state by a time-dependent force which, however, derives from a time-dependent potential. This means that the driving force, if constant in time, does not exert work upon the system whatever its intensity $\epsilon$. This class of systems includes spinglass models in a oscillating magnetic field and tapped granular systems where the relevant parameter is the frequency of the driving force. These systems are described in section 7.4 .2

7.4.1. Sheared systems Studies of models described by non-conservative forces go back to the study of neural network models described by synaptic interactions that are non-symmetric [283. This has inspired future investigations of disordered models where couplings among spins include an important degree of asymmetry. In [284, 285] 
it was considered the relaxational dynamics of the $p=2$ spherical spin-glass with pairwise interactions $J_{i j}$ given by $J_{i j}=J_{i j}^{S}+\epsilon J_{i j}^{A}$ where $J_{i j}^{S}=J_{j i}^{S}$ denotes the symmetric (therefore conservative) part and $J_{i j}^{A}=-J_{i j}^{A}$ stands for the antisymmetric (therefore non-conservative) part. It was shown that any finite amount of asymmetry is enough to destroy the spin-glass phase. The relaxational time of the system was found to diverge as $\epsilon^{-6}$ for $\epsilon \rightarrow 0$. Importantly, this result suggests the following general scenario: whatever the intensity of the non-conservative force, the stationary state has a finite relaxation time, therefore the stationary state, although of nonequilibrium nature, must be TTI (and therefore, correlations and response functions do not display aging). Subsequent investigations have confirmed this result showing that this is a generic feature of driven systems.

Among this family of asymmetric spin-glass models, one which has been intensively investigated in the past years is the $p$-spin spherical spin-glass Hamiltonian (150), (151) with asymmetry in the interactions. The model is defined according to the following Langevin dynamics [286, 287]

$$
\frac{\partial \sigma_{i}(t)}{\partial t}=-r(t) \sigma_{i}(t)+F_{i}(\{\sigma\})+\eta_{i} .
$$

This dynamics is the same as (153) but now the force $F_{i}(\{\sigma\})$ is replaced by,

$$
\begin{aligned}
F_{i}(\{\sigma\}) & =-\frac{\delta \mathcal{H}}{\delta \sigma_{i}}+F_{i}^{\text {drive }}(\{\sigma\}) \\
& =-\frac{\delta \mathcal{H}}{\delta \sigma_{i}}+\epsilon \sum_{j_{1}<. .<j_{k-1}} K_{i}^{j_{1}, j_{2}, . ., j_{k-1}} \sigma_{j_{1} . . \sigma_{j_{k-1}}}
\end{aligned}
$$

where $\mathcal{H}$ is the Hamiltonian given in (150) and the driving force $F_{i}^{\text {drive }}(\{\sigma\})$ describes a $k$-spin interaction term where the couplings $K_{i}^{j_{1}, j_{2}, \ldots, j_{k-1}}$ are uncorrelated among all permutations of the $k$ different indexes $\left(i, j_{1}, j_{2}, . ., j_{k-1}\right)$,

$$
\overline{K_{i}^{j_{1}, j_{2}, \ldots, j_{k-1}} K_{j_{r}}^{j_{1}, j_{2}, \ldots, j_{r-1}, i, j_{r+1}, \ldots, j_{k-1}}}=0
$$

These models show the following behavior. In the regime above the mode-coupling temperature $T_{\mathrm{c}}$, the relaxation time is finite for the unsheared model $\epsilon=0$. Therefore both TTI and FDT hold in the stationary state. A small driving force $\epsilon>0$ puts the system in a new stationary state where TTI holds but FDT is violated. The numerical analysis of the mean-field equations 287 reveals that both FD plots and the value of the FDR are very similar to those found in aging systems. Figure 31]shows these quantities above $T_{\mathrm{c}}$ for the model with parameters $k=p=3$. Below $T_{\mathrm{c}}$ a new phenomenon, called "shear thinning" occurs. At $\epsilon=0$ the relaxation time diverges (as is common in mean-field (MF) models where activated processes are neglected, see discussion in section 6.1). However, as $\epsilon>0$ the relaxation time becomes finite and decreases with $\epsilon$ (shear thinning). Again, numerical analysis of the mean-field equations reveals that for finite $\epsilon$ the resulting FD plots are the same as for an aging system with a waiting time $t_{w}$ given by $t_{w} \sim \epsilon^{-\alpha(T)}$ where $\alpha(T)$ is a temperature dependent exponent that takes the value 2 at $T_{\mathrm{c}}$ and slowly increases as $T$ decreases. This scenario, as derived from the study of MF spin-glass models, has been confirmed in numerical studies of binary mixtures of Lennard-Jones (LJ) sheared fluids (see section 7.1.2). In a series of papers, Barrat and Berthier 288, 289, 290 have shown that driven short-range systems display the same features as their equivalent MF disordered models. These similarities have been confirmed by other studies that measure the temperature dependence of the shearing rate at which the average potential energy 


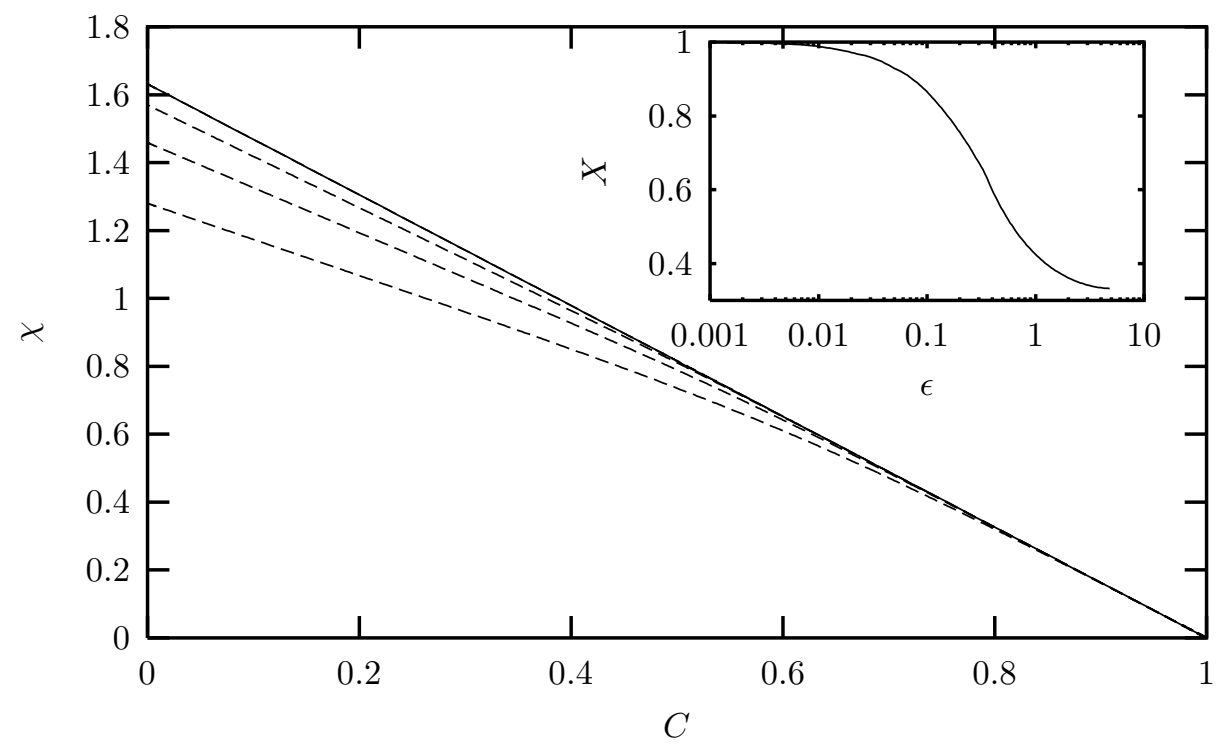

Figure 31. FD plot for the model 261, 262, 263 with $k=p=3$ and $T=0.613>T_{\mathrm{c}}=0.612$. The full line is the $\epsilon=0$ equilibrium curve. Dashed lines correspond (from bottom to top) $\epsilon=0.333,0.143,0.05,0$. The inset is the FDR as function of the intensity of the perturbation. From [287.

deviates from its equilibrium value 291]. The similarity between LJ models and MF spin glasses is striking concerning rheological properties. In a sheared fluid the velocity field $\boldsymbol{v}=\dot{\gamma} y \boldsymbol{e}_{x}$ induces a stress $\sigma_{x y}$ that is well described by the phenomenological law: $\sigma_{x y}=\sigma_{0}+a \dot{\gamma}^{n}$. This law is commonly found in rheological systems 292]. The viscosity is defined by $\eta=\sigma / \dot{\gamma}$ : for Newtonian fluids $n=1$ and $\sigma_{0}=0$, the viscosity is therefore independent of the stress. However, this is known to be inaccurate as many complex fluids show transport coefficients (such as the viscosity) that depend on the shear rate (for a discussion of these effects in the framework of non-equilibrium thermodynamic theories see 293,294 ). In figure 32 we show the flow curves for the viscosity as function of the shear rate for both the LJ fluid and the MF spin-glass, the viscosity $\eta$ and the shear $\dot{\gamma}$ in the LJ model corresponding to the terminal relaxation time $\tau_{\alpha}$ and $\epsilon / \tau_{\alpha}$ in the MF spin glass respectively. The similarity is noteworthy. There are two regimes depending whether $T>T_{\mathrm{c}}$ or $T<T_{\mathrm{c}}$. In the first regime the fluid is Newtonian at low shearing rates so the viscosity is shear independent. In this regime, standard non-equilibrium thermodynamics 295] is applicable. However, for $T<T_{\mathrm{c}}$ the fluid is non Newtonian and the viscosity diverges at zero shear, $\eta \sim \dot{\gamma}^{-\alpha(T)}$ with $\alpha(T)$ between $2 / 3$ (at $T_{\mathrm{c}}$ ) and 1 (for $T \rightarrow 0$ ) in agreement with the aforementioned results found in the MF spin glass. The central question addressed in [288, 289, 290] was the dependence of the resulting FD plots on the type of observable used as a perturbation. They considered 5 different classes of observables: 1) The incoherent part of scattering functions (corresponding to single particle density fluctuations), 2) the coherent part or the correlations, 3) The "chemical" observables associated to correlations of a single species of particles in the binary mixture, 4) The mean-square 

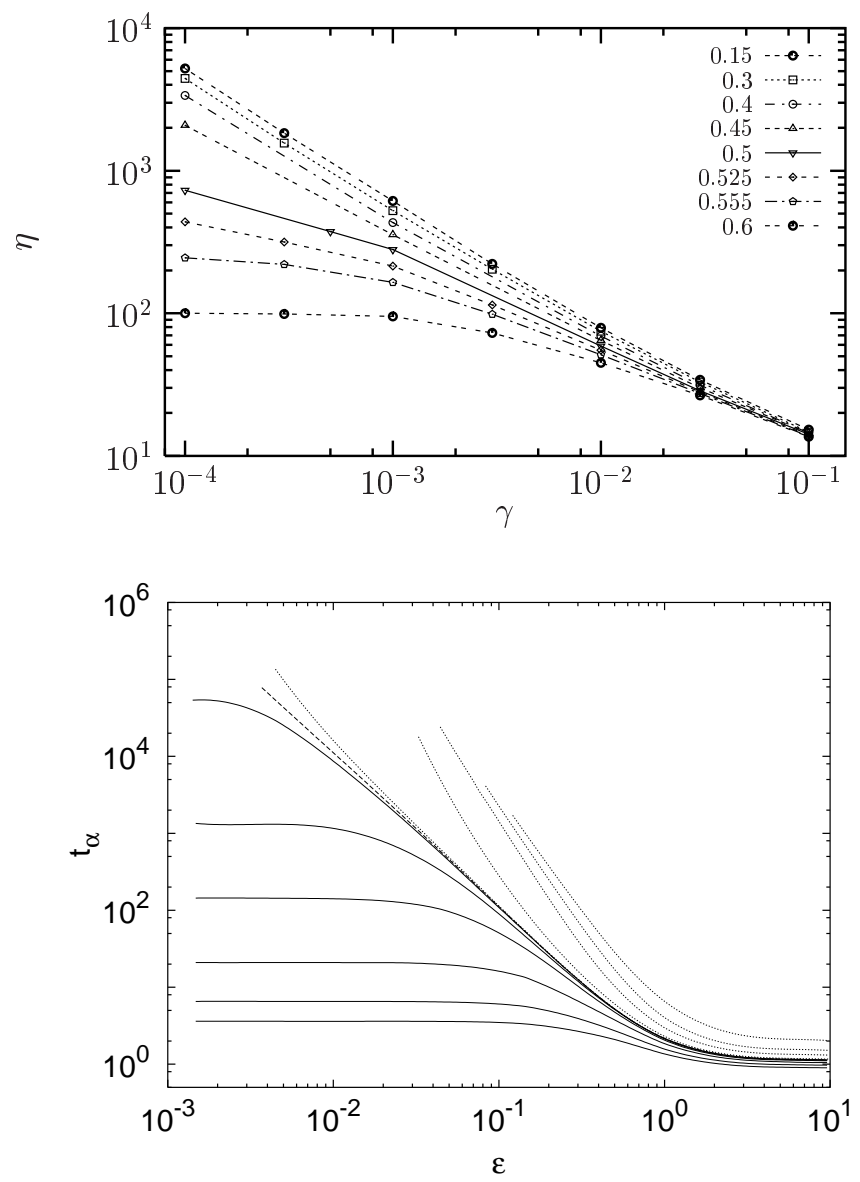

Figure 32. Viscosity as a function of the shear rate for the LJ (top panel) and MF spin-glass models (bottom panel). The viscosity $\eta$ and shear rate $\dot{\gamma}$ in the LJ fluid correspond to the terminal time $\tau_{\alpha}$ and $\epsilon / \tau_{\alpha}$ respectively in the MF model. $T_{\mathrm{c}} \simeq 0.435$ in the LJ model and $T_{\mathrm{c}} \simeq$ 0.612 in the MF model. Temperatures are (from bottom to top): (bottom panel) $0.9,0.8 .0 .7,0.64,0.62,0.613, T_{\mathrm{C}}, 0.6115,0.58,0.45,0.3,0.01$ and (upper panel) as indicated in the box. From [287] (left panel) and 289.

displacement of particles associated to a constant small force transverse to the flow and 5 ) the stress in the transverse direction after compression of the box. 1), 2) and 3) were measured at different wavevectors. In all cases, the effective temperature was found to be the same within numerical accuracy. A compendium of their results is shown in figure 33 There have been other studies on driven systems that have investigated the shear thinning effect, i.e. whether a driving force of rate $\dot{\gamma}$ stops aging up to a timescale proportional to $1 / \dot{\gamma}$ by restoring TTI. Corberi et al. 296] have investigated coarsening models in the presence of a driving force. In particular, they have considered a $\mathrm{N}$ component ferromagnetic model with non-conserved order parameter in the large $N$ limit. The authors find that, above the ferro-paramagnetic transition temperature $T_{\mathrm{c}}$, the inverse of the driving rate $1 / \dot{\gamma}$ sets the time scale after which aging stops and the system becomes TTI. However, below $T_{\mathrm{c}}$, contrarily to what is observed in MF spin 


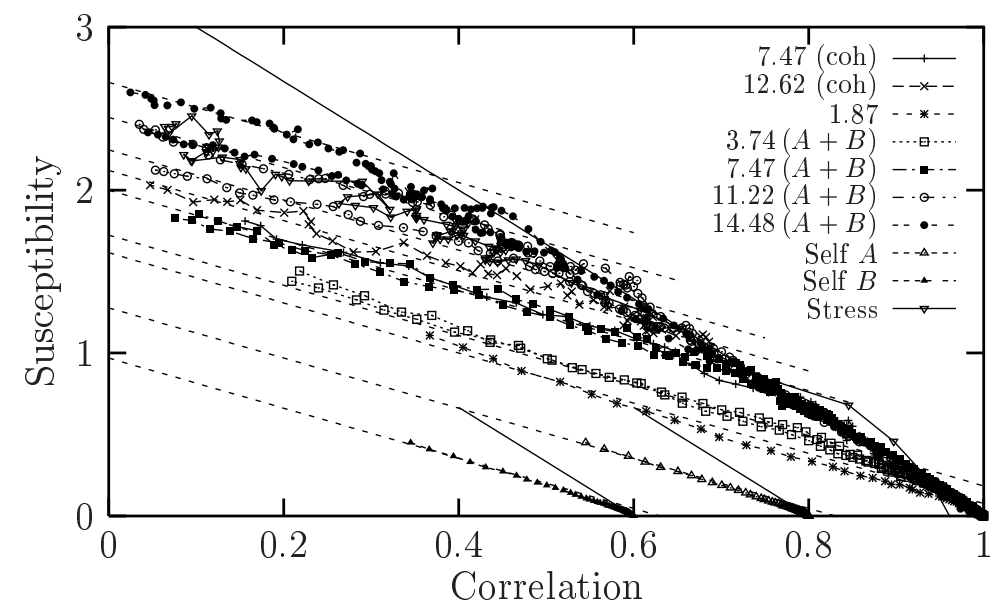

Figure 33. $14 \mathrm{FD}$ parametric plots in the LJ model showing the independence of the effective temperature on the observable. The numbers in the key of the figure indicate different values of the wavevectors. 1.87 refers to class 1 in the text. coh stands for coherent or global scattering functions (class 2). $A$ and $B$ refer to the two different chemical species, $(\mathrm{A}+\mathrm{B})$ standing for incoherent scattering observables independently measured for $A$ and $B$ species (class 3 ). self indicates the use of the mean-squared displacement (class 4). stress refers t class 5 . The value of the effective temperature is compatible with $T_{\text {eff }}=0.65>T=0.3$ for all cases. From 289].

glasses, aging never stops even in the presence of shearing. The origin of this difference is unclear. It could be related to the fact that in coarsening systems the mechanism responsible of aging is different than in MF spin glasses or LJ glasses. This difference can be traced back to the absence of complexity in the coarsening model as compared to the other cases. Moreover, this behavior has to be contrasted with what is found in tapped systems such as MF spin glasses in an AC field [297] where aging survives below $T_{\mathrm{c}}$ in a certain range of magnetic field values (see further discussion on this model in section (7.4.2). Other, studies have considered models such as vortex glasses with random pinning centers in two dimensions [298] where the external driving force is uniform over all vortices. Also in this case FDT violations appear to be described by an effective temperature related to the slow motion of vortices. Trapping dynamics due to pinning defects has also been considered in the study of a driven classical particle submitted to a force [299, 300]

A common feature of all studies reported here, that has not been emphasized enough in the existing literature, is that measured correlations must be transverse to the direction of the shear flow to yield a meaningful effective temperature. FDT violations for longitudinal observables are apparently not described by an effective temperature. This suggests that neutral observables describing an effective temperature are restricted to the transverse direction, a fact that lacks yet a clear explanation.

7.4.2. Tapped systems As already said, another class of driven systems correspond to those where the external force derives from a time-dependent potential that pumps 
energy into the system. This includes also driven granular media that have recently received considerable attention.

In 297, the authors have studied the MF $p$-spin glass model in an oscillating AC field. This problem is interesting as it shows a behavior different to that observed in sheared MF spin glasses or LJ glasses (see section [7.4.1). The model considered was again (150), (151) but in the presence of an uniform AC field of frequency $\omega$ and intensity $h$. The phase diagram of the model is described by three parameters $T, h, \omega$. Below $T_{\mathrm{c}}$, at fixed $\omega$, the glassy phase survives below a critical field $h_{\omega}^{*}(T)$ meaning that, contrarily to sheared systems, a small driving force does not destroy the glassy phase and aging never stops, hence TTI is not restored. This justifies the use of AC fields in experiments to explore the aging regime within the linear response region. A striking result in these studies is the presence of reentrant behavior at constant field $h$, as $\omega$ is varied, indicating that $\lim _{\omega \rightarrow 0} h_{\omega}^{*}(T, \omega)<h_{\omega}^{*}(T, \omega=0)$, a result that needs yet to be clarified. All over the glassy phase of the driven model FDT is violated with the characteristic FD plots of the corresponding relaxational model.

Granular systems may present very slow processes, analogous to what is seen in glasses. Similar to what has been done for other glassy systems one can try to describe the dynamics of the slow degrees of freedom through an effective temperature defined from the FDR [100]. The first example we consider is the kinetically constrained Kob-Andersen model [301] that, even if very schematic, reproduces rather well several aspects of glasses 302 and of granular compaction [303. Although next section 7.5 is devoted to kinetically constrained models we prefer to describe the Kob-Andersen model here since it is a good model for granular media. This model consist of $N$ particles in a cubic lattice, with periodic boundary conditions. There can be at most one particle per site. Apart from the hard-core repulsion there are no other static interactions among particles. At each time step a particle can move to a neighboring empty site of a three-dimensional lattice only if it has less than four neighbors in the initial and final position. In its simpler version there is no gravity, but the system is subject to a constant pressure on its surface, obtained by adding or destroying particles on the topmost layer with a chemical potential $\mu$. The dynamic rule guarantees that at equilibrium all configurations of a given density are equally probable. Indeed when the density are of the order of the the jamming density $\rho_{g} \simeq 0.88$ the particle diffusion becomes extremely slow, due to the kinetic constraints, slowing down the whole compaction process. As done for glasses, one can try to describe the slow non-equilibrium motion through an effective dynamical temperature $T_{\text {eff }}$ which can be defined via a generalization of the Einstein-Stokes relation between the diffusion coefficient and the viscosity to a non-equilibrium (aging) situation:

$$
\frac{\partial}{\partial \boldsymbol{f}}\left\langle\boldsymbol{r}(t)-\boldsymbol{r}\left(t_{w}\right)\right\rangle=-\frac{X(B)}{2 T} \frac{\partial}{\partial t_{w}}\left\langle\left(\boldsymbol{r}(t)-\boldsymbol{r}\left(t_{w}\right)\right)^{2}\right\rangle
$$

where $\boldsymbol{r}$ is the particle position, $\boldsymbol{f}$ a (small) perturbing field, $t>t_{w}$ two widely separated times, and $B\left(t, t_{w}\right)$ the mean square displacement:

$$
\begin{aligned}
B\left(t, t_{w}\right) & =\left\langle\left(\boldsymbol{r}(t)-\boldsymbol{r}\left(t_{w}\right)\right)^{2}\right\rangle \\
& =\frac{1}{3 N} \sum_{a=1}^{3} \sum_{i=1}^{N}\left\langle\left[r_{i}^{a}(t)-r_{i}^{a}\left(t_{w}\right)\right]^{2}\right\rangle
\end{aligned}
$$

The linear response function can be computed numerically by applying a small random 


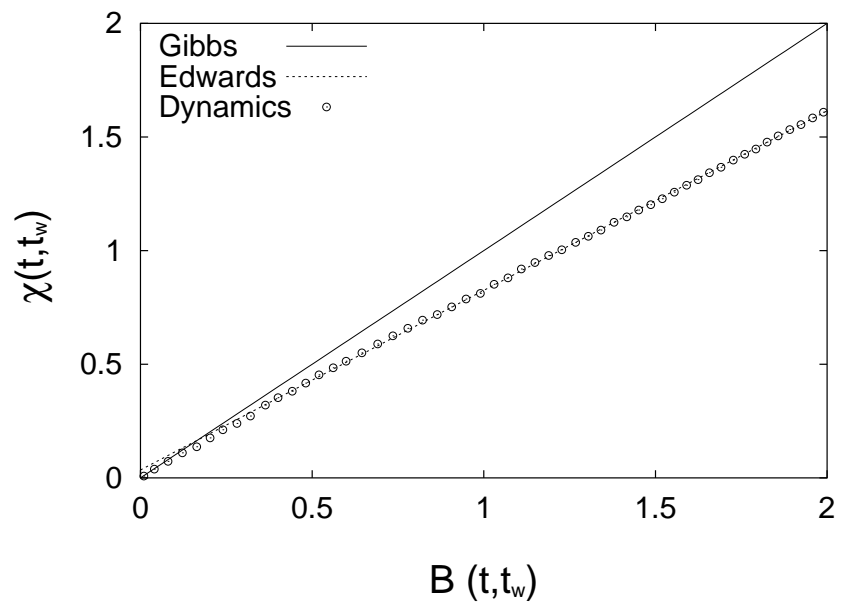

Figure 34. Einstein relation in the Kob-Andersen model: plot of the mobility $\chi\left(t, t_{w}\right)$ vs. the mean-square displacement $B\left(t, t_{w}\right)$ (data shown as circles). The slope of the full straight line corresponds to the equilibrium temperature $(T=1)$, and the slope of the dashed one to Edwards' prescription obtained from figure 35 at $\rho\left(t_{w}\right)=0.848$. From 305.

perturbation at time $t_{w}$ of the form 304:

$$
\delta \mathcal{H}_{\epsilon}=\epsilon \sum_{a=1}^{3} \sum_{i=1}^{N} f_{i}^{a} r_{i}^{a}
$$

where $f_{i}^{a}$ are independent quenched random variables which take the value \pm 1 with equal probability. With this choice the IRF is defined as:

$$
\chi\left(t, t_{w}\right)=\frac{1}{3 N} \sum_{a=1}^{3} \sum_{i=1}^{N}\left(\left\langle f_{i}^{a} \Delta r_{i}^{a}(t)\right\rangle\right.
$$

where $\Delta \boldsymbol{r}\left(t+t_{w}\right)$ is the difference between the displacement of the same particle in two identical copies of the system one evolving in presence of the external perturbation and one without. From (264) $\chi$ is related to $B$ through;

$$
\begin{aligned}
\chi\left(t, t_{w}\right) & =\frac{\epsilon}{2 T} \int_{0}^{B\left(t, t_{w}\right)} X(B) d B \\
& =\frac{\epsilon}{2 T_{\text {eff }}} B\left(t, t_{w}\right), \quad \text { if } X(B)=m=\text { const }
\end{aligned}
$$

with $T_{\text {eff }}=T / m$. The numerical simulations show a two regime scenario 304] similar to what is seen in glasses, i.e., for $t-t_{w}$ smaller than $t_{w} m=1$ and FDT holds, while for $t-t_{w} \gg t_{w}$ FDT is violated with $m<1$, figure 34 As discussed in section 5.3 the value of the observables attained dynamically in a granular system could be computed from the usual equilibrium microcanonical distribution at the corresponding density $\rho$ restricted to the subset of blocked configurations (Edwards ensemble), i.e., only to those configurations in which every grain is unable to move. Much alike to the IS analysis in glassy systems (section 4.5), the Edwards ensemble leads to the definition a temperature, called the Edwards' temperature, as

$$
\frac{1}{T_{\mathrm{Edw}}(\rho)}=-\frac{1}{\mu} \frac{\partial s_{\mathrm{Edw}}(\rho)}{\partial \rho}
$$


where $s_{\mathrm{Edw}}(\rho)$ is the Edwards' entropy density obtained from the logarithm of the number of blocked configurations of given $\rho$, see (135). The chemical potential fixes the dimension [306, 305].

The Edwards' entropy for this model has been computed in 306, 305 through the use of an auxiliary model in which each particle has energy equal to one if the dynamic rules allow it to move, and zero otherwise. The auxiliary energy $E_{\text {aux }}$ is hence equal to the number of mobile particles. The configurations of the auxiliary model are sampled with a Monte Carlo procedure with non-local moves at the auxiliary temperature $1 / \beta_{\text {aux }}$. These non-local moves have nothing to do with the true dynamics of the original model, and the auxiliary model is not glassy. This allows to obtain equilibrium properties such as the auxiliary energy density $e_{\text {aux }}$ from which the entropy density can be obtained via thermodynamic integration:

$$
\begin{aligned}
s_{\text {aux }}\left(\beta_{\text {aux }}, \rho\right)= & s_{\text {equil }}(\rho)+\beta_{\text {aux }} e_{\text {aux }}\left(\beta_{\text {aux }}, \rho\right) \\
& -\int_{0}^{\beta_{\text {aux }}} e_{\text {aux }}\left(\beta_{\text {aux }}^{\prime}, \rho\right) d \beta_{\text {aux }}^{\prime}
\end{aligned}
$$

since $s_{\text {aux }}(0, \rho)=s_{\text {equil }}(\rho)$. The blocked configurations at a given density can be computed performing a simulated annealing down to $\beta_{\text {aux }} \rightarrow \infty$ of the auxiliary model at fixed particle density. The Edwards' entropy density is then obtained as

$$
\begin{aligned}
s_{\text {Edw }}(\rho) & =\lim _{\beta_{\text {aux }} \rightarrow \infty} s_{\text {aux }}\left(\beta_{\text {aux }}, \rho\right) \\
& ==s_{\text {equil }}(\rho)-\int_{0}^{\infty} e_{\text {aux }}\left(\beta_{\text {aux }}, \rho\right) d \beta_{\text {aux }} .
\end{aligned}
$$

since $e\left(\beta_{\text {aux }}, \rho\right)$ vanishes for $\beta_{\text {aux }} \rightarrow \infty$. The Edwards' entropy is shown in figure 35] as function of density. We are now in a position to compare the long-time nonequilibrium results with those obtained from the Edwards' measure. In figure 34 it is shown the mobility $\chi\left(t, t_{w}\right)$ versus the mean square displacement $B\left(t, t_{w}\right)$. The agreement between $T_{\text {eff }}$ and $T_{\mathrm{Edw}}$ is clearly satisfactory.

Similar results have been found [306, 305, 307] also for models with geometrical, rather than kinetical, constrains, the so called "Tetris" models [308, 309. These models are defined on a two-dimensional lattice with particles of randomly chosen shapes an sizes. The only constraint is that particles cannot overlap: for two nearest neighbor particles the sum of the arms oriented along the bond connecting them has to be smaller that the bond length.

Tapped granular matter [310, 311] has been considered in several models such as one-dimensional model ferromagnet [312] showing that the Edwards measure provides a very good description of the stationary state. A similar agreement is found in simulations of sheared granular matter [313]. Real compaction occurs in presence of gravity [314. The gravity introduces an extra term in the Hamiltonian

$$
\mathcal{H}_{g}=m g \sum_{i=1}^{N} h_{i}
$$

where $g$ is the gravity constant, $m$ the particle mass and $h_{i}$ the eight of $i$-th particle. The simplest way of including gravity in the above models is assuming that particles can move up and down (if they respect the geometrical or kinetical constraints) with different probability, $p=\min [1, \exp (-m g \Delta h / T)]$ where $\Delta h=-1,0,1$ is the elementary vertical displacement. A closed boundary is situated at the bottom of the system. The control parameter $x=\exp (-m g / T)$ represents the "vibration". 


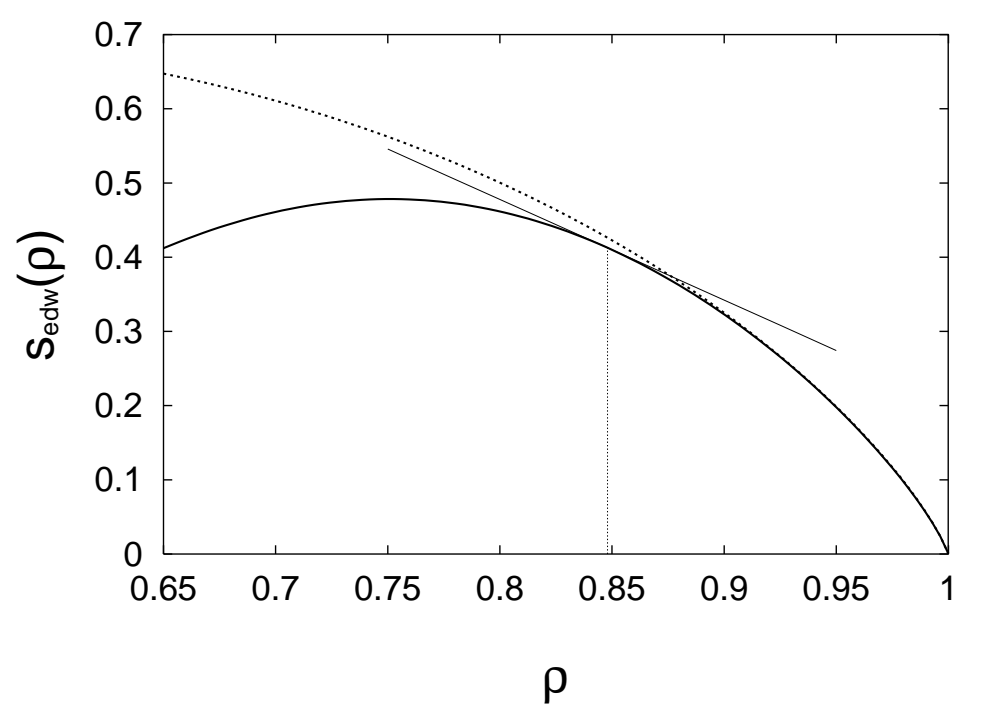

Figure 35. Edwards entropy per particle of the Kob-Andersen model vs. density (full curve). For comparison we also show the equilibrium entropy (dashed curve). At high enough density the curves are indistinguishable, and join exactly only at $\rho=1$. The slope of the tangent to $s_{\mathrm{Edw}}(\rho)$ for a generic $\rho$ allows to extract $T_{\mathrm{Edw}}(\rho)$ from the relation $T_{\mathrm{Edw}}(\rho) \frac{d s_{\mathrm{Edw}}}{d \rho}=\mu$. From [305].

The presence of gravity introduces a preferred direction in the diffusive motion (downward) which in turn may produce inhomogeneities in the vertical density profile making the situation more complicated, moreover horizontal (transversal) and vertical (longitudinal) quantities must be treated separately.

Inhomogeneities are only along the vertical direction, so transversal observables when measured well inside the bulk are not too sensitive to the detailed form of the density profile. Indeed if homogeneity of the bulk is imposed, the dynamical temperature obtained from the FDT ratio for the horizontal displacement-mobility coincides with the Edwards' temperature 307.

The analysis of the FDT ratio for the longitudinal motion [315 316 also shows a two-slope scenario, however the comparison with the Edwards' measure is more complex due to the presence of inhomogeneities. In [316, based on the observation that the density profile far from the top and bottom layers is rather flat, the effective dynamical temperature for longitudinal observables and the Edwards' temperature have been compared assuming an homogeneous density. The comparison, however, reveal strong deviations when inhomogeneities are stronger indicating that inhomogeneities of the density profile must be included. This could be done by using the recent introduced restricted Edwards measure [116, 317, a route not yet explored.

We stress that the Edwards' measure is constructed from a white sampling of blocked configurations, hence it reproduces the physical quantities at large times only if this condition is satisfied by the long-time dynamics. In other words the Edwards' measure can be inappropriate even though the system presents a slow dynamics. This is for example the case of the three-dimensional Ising model in a weak random magnetic field 306, 305]. Here the long-time configurations at low temperatures are made of domains of "up" and "down" spins of similar volumes, so that the global magnetization 
is zero. This is quite different from either the equilibrium or blocked configurations since both of them are magnetized. Other studies have extended this method to the study of kinetically constrained models (KCMs) (see section 7.5) such as the KobAndersen model 318.

\subsection{Kinetically constrained models}

A category of statistical models that has received considerably attention during the last years are kinetically constrained models $(\mathrm{KCMs})$. In a nutshell, $\mathrm{KCMs}$ are models with trivial thermodynamics but complicated dynamics arising from a, put by hand, set of forbidden transitions in configurational space. Allowed transitions are selected according to a given transition rule that constrains the dynamics of the system. There are different ways to justify $\mathrm{KCMs}$ as valuable models for glassy dynamics. One can think of KCMs as effective models in which the slowest degrees of freedom are idealized as quenched variables that manifest as dynamical constraints. Therefore, the statistical variables of KCMs can be seen as the fast dynamical variables that are slaved, through the dynamical constraints, to the motion of the slowest ones. These constraints are of local nature as also is the interaction between the original degrees of freedom.

KCMs display most (if not all) of the features characteristic of glassy systems including slow relaxation, activated behavior, cooperativity and non-equilibrium phenomena such as aging and FDT violations. Recently, a review on KCMs has been written that covers all these aspects. For this reason, here in this review we will not dwell much on discussing FDT violations on these models but content ourselves on underlining some of the most important results. We refer the reader to 15 for a comprehensive and exhaustive survey.

The most representative families of KCMs are spin-facilitated models [319, 320], lattice gases 301, 321, 302, 322, 304, topological cellular models 323, 324, and plaquette models 325, 326, 327. We already discussed in section 7.4 .2 the KobAndersen model as an example of kinetically constrained lattice gas for granular matter. There are two aspects of KCM's that makes them specially attractive from a theoretical point of view. On the one hand, the thermodynamics of KCMs is straightforward, there is no underlying thermodynamic singularity, and even more astonishing, often the model is purely non-interacting from the point of view of its equilibrium properties. On the other hand, the non-interacting character of the energy function, entails that the slow dynamics is described by representing the original variables in terms of a new set of effective variables. Relaxation can be visualized as a dynamical process where defects diffuse (in either a free or cooperative way) and annihilate each other, leading to a more tractable problem from the analytical point of view.

We begin our tour presenting the simplest among these type models. Our intention is to illustrate with an example what type of models KCMs are. Maybe the simplest KCM is the spin facilitated model (SFM) introduced by Fredrickson and Andersen (FA) model 319, 320 consisting of free "spins" in a field. The model is defined by,

$$
E=h \sum_{i=1}^{N} n_{i}
$$

where $n_{i}=0,1$ corresponding to two possible orientations of the spin ( $n=1 \mathrm{up}, n=0$ 
down) $\dagger$ that occupy a D-dimensional lattice. This model would be trivial if it were not by the dynamical rules that describe how spins can flip. In the standard SFM model transitions $n_{i} \rightarrow 1-n_{i}$ are allowed if at least $f$ among the possible nearest neighbours are up or $n=1$. This model defines the $f, d$-SFM that shows different behaviors according to whether $f=1$ (diffusive) of $f>2$ (cooperative). Variations of the SFM include KCMs with directed constraints such as the 1d East model [328] or the 2d North-East model [329], defined again by (273), but where a spin $n_{i}$ can flip only if the spin on its left is up (East model) and if both spins, that are nearest neighbours in two fixed orthogonal directions (North-East model), point up. In 1d, it is also possible to define a model that interpolates between the 1,1-SFM and the East model [330.

In what follows we concentrate our attention in FDT violations in diffusive $\mathrm{KCMs}$ where some understanding has been recently gained. A generic aspect of KCM's is that dynamics is determined by the motion and annihilation of isolated defects. Slow dynamics strictly occurs at temperatures close to $T=0$ (and timescales larger than an initial fast transient). The relevant relaxing variable is the number of isolated defects $c(t)$ (e.g. in the 1,1-SFM this corresponds to the number of up spins). This has interesting consequences [327, 217, 331] in the behavior of the IRF. The IRF in the presence of an external random staggered field $\chi\left(t, t_{w}\right)$ is then proportional to the product of the number of isolated defects $c(t)$ and the individual local response typical of one defect $\chi_{\text {eff }}$. This local response is TTI as the defect is isolated leading to,

$$
\chi\left(t, t_{w}\right) \simeq \chi_{\mathrm{ag}}\left(t, t_{w}\right)=c(t) \chi_{\mathrm{eff}}\left(\frac{t-t_{w}}{\tau(T)}\right)
$$

where $\tau(T)$ is the relaxation time of the defect. This relation has two important consequences: 1) The function $\chi\left(t, t_{w}\right) / c(t)$ is TTI, a result which is characteristic of coarsening systems (see below) but not of other (cooperative) glassy models where the IRF is a genuine aging function depending, for instance, on the ratio $h(t) / h\left(t_{w}\right)$ and 2) $\chi\left(t, t_{w}\right)$ has a maximum as function of $t-t_{w}$ when the defect concentration (that decreases with time) compensates the growth of the monotonically increasing effective response $\chi_{\text {eff. }}$.

Non-equilibrium measurements for the 1,1-SFM 70,332 and related models such as the 2d triangle model 333, 334 or topological cellular models 323, 324] show an IRF (274) displaying a maximum as a function of $t-t_{w}$ and leading to awkward FD plots. One example is shown in figure 36] Buhot and Garrahan 327 have explained how to recover well defined FD plots by using (274) and plotting $\chi\left(t, t_{w}\right)$ as function of the difference $C_{c}(t, t)-C_{c}\left(t, t_{w}\right)$ where $C_{c}$ is the standard connected correlation function $C_{c}(t, s)=\langle(n(t)-c(t))(n(s)-c(s))\rangle$ with $c(t)=\langle n(t)\rangle$ for the 1, 1-SFM. In this case, the resulting FD curve corresponds to a straight line (corresponding to the equilibrium result) casting doubts on the usefulness of FD plots for non-cooperative models.

The origin of the maximum in the IRF has also been considered in the 1d RFIM with infinite-ferromagnetic coupling $J=\infty$ 331. Actually, in this limit the model turns out to be a KCM as transitions are only allowed on the spins sitting on the interfaces of the ferromagnetic domains, i.e. a spin can flip only if its left and right

$\dagger$ The variables $n$ cannot be considered as occupancies and 273 does not define a lattice gas model. In lattice gas models the total number of particles (i.e. the energy $E$ in (273) is conserved while in the SFM it is not. See [15] for a throughout discussion. However, we will continue denoting the spins by $n$. 


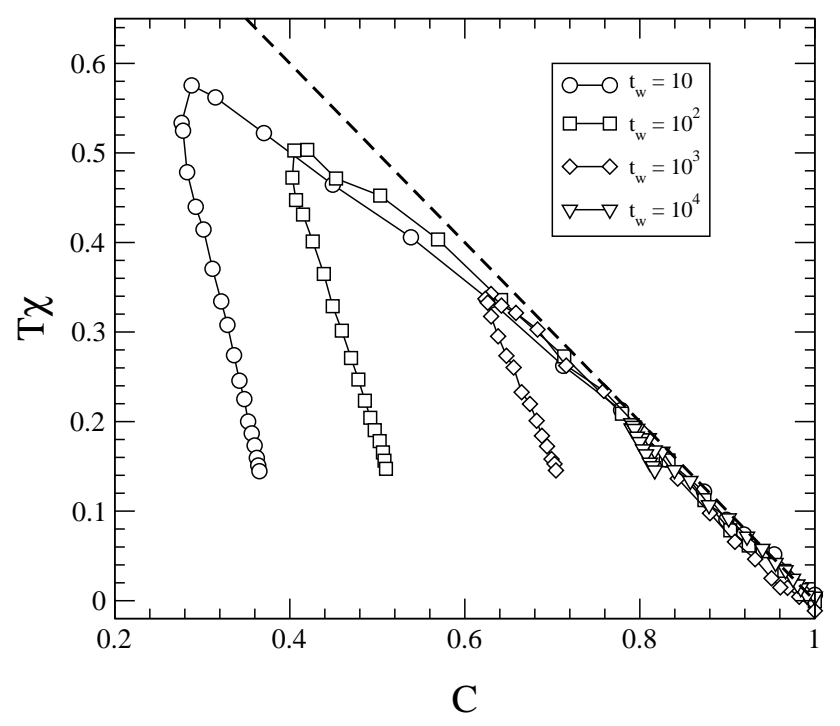

Figure 36. FD plots for the 1, 1-SFM 273) with $N=10^{5}$, quench temperature $T=0.3$ and different waiting times (see the box). The straight line is the FDT relation. From [0].

neighbours point in different directions (meaning that the ferromagnetic contribution to the local field acting on that spin vanishes). If quenched at low enough temperature this model displays a coarsening behavior with two well separated regimes. If the average distance between interfaces (or average domain length) $L(t)$ is smaller than a length scale $L_{g} \sim\left(T / h_{0}\right)^{2}$ (where $h_{0}$ is the mean square deviation of the intensity of the random field) then dynamics is diffusive. However, if $L(t)>L_{g}$ dynamics becomes activated of the Sinai type. In the Sinai regime the IRF is well described by the relation (274), $\chi\left(t, t_{w}\right)=\chi_{\mathrm{ag}}\left(t, t_{w}\right)=c(t) \chi_{\mathrm{eff}}\left(t, t_{w}\right)$ leading to a maximum of the IRF at intermediate times where $L(t) \sim L_{g}$. This relation sets a crossover timescale where pure diffusion takes over to Sinai diffusion. Comparing this result with (70) we note that there is no stationary contribution to the IRF because for $J=\infty$ thermal fluctuations within domains are suppressed. Again this leads to FD plots similar to figure 36. For the 1d RFIM in the asymptotic long-time limit $L(t) \gg L_{g}$ the $\chi\left(t, t_{w}\right)$ decays to zero, a property required to establish a link between static and dynamic properties. As remarked in section 6.6. however, this property does not hold for the 1d Ising model.

A description of the IRF for the $2 \mathrm{~d}$ plaquette model along the same lines of (274), but modified to account for the diffusion and annihilation of oscillating pairs of defects, has been presented in [327. The resulting FD plots have been shown to display the characteristic two slope curves of two-timescale systems. However, it is unclear what is the physical meaning of the piece of the curve with slope $X<1$ and whether indeed $T_{\text {eff }}=T / X$ can be considered as a thermodynamic temperature. Future research will show what is the true meaning of these violation factors in KCMs of the coarsening type.

FDT violations in the glassy regime of KCMs are not simply described within a thermodynamic IS formalism, the Kob-Andersen model perhaps being an exception. In fact, the blocked states in the 1,1-SFM and the East model generate identical 
configurational entropies [70] albeit they show very different dynamics (diffusive and cooperative respectively). The applicability of thermodynamic non-equilibrium concepts to models with trivial equilibrium thermodynamics remains an open question.

\section{QFDT: the experimental evidence}

Any valuable physical theory must be successfully challenged by experiments. Traditionally, the most direct way to experimentally access FDT violations is through noise measurements. In the frequency domain the FDT (60) corresponds to the Nyquist formula that relates the power spectrum of an observable to the imaginary part of its susceptibility. With the same notation we used in sections [213] we can write define the power spectrum $S_{A, B}(\omega)$ and the complex susceptibility $\chi_{A, B}(\omega)$,

$$
\begin{aligned}
& S_{A, B}(\omega)=\frac{1}{\pi} \int_{-\infty}^{\infty} C_{A, B}(t) \exp (i \omega t) d t \\
& \chi_{A, B}(\omega)=\frac{\delta\langle\widehat{A}(\omega)\rangle}{\delta B(\omega)}
\end{aligned}
$$

where $\widehat{A}(\omega)$ (and analogously $B$ ) is given by,

$$
\widehat{A}(\omega)=\int_{-\infty}^{\infty} A(t) \exp (i \omega t) d t
$$

If $\chi_{A, B}(\omega)=\chi_{A, B}^{\prime}(\omega)+i \chi_{A, B}^{\prime \prime}(\omega)$ the Nyquist formula (133) reads [295],

$$
S_{A, B}(\omega)=\frac{2 k_{B} T}{\pi} \frac{\chi_{A, B}^{\prime \prime}(\omega)}{\omega}
$$

The power spectrum can be experimentally measured considering the case where the external perturbation couples to the measured observable $A=B$. In the experimental protocol, the time evolution of the observable $A(t)$ and the out-of-phase susceptibility $\chi_{A, B}^{\prime \prime}(\omega)$ are recorded. The power spectrum is given by $S_{A}(\omega) \propto\left\langle|\hat{A}(\omega)|^{2}\right\rangle$. This allows to verify the Nyquist relation (278). Typical noise experiments are the observation of electric voltage fluctuations or the motion of a Brownian particle. Other measurements included sample to sample fluctuations of the resistivity in small samples [335, 336]. Observations of magnetic noise were successfully undertaken in spin-glasses [337, 338, 339, more than ten years ago, and despite of the extremely low noise signal characteristic of magnetic systems. Globally, these experiments show that, within the accessible window of frequencies and times, no systematic FDT violations are observed. The out-of-phase susceptibility $\chi^{\prime \prime}(\omega)$ associated to $\chi(\omega)$ is practically frequency independent and the power spectrum shows the characteristic $1 / f$ behavior. The negative result of these experiments points out one of the most important difficulties encountered in these type experiments. As they cover the frequency range $10^{-2}, 10^{2}$ Hertz, these frequencies are much larger than the inverse of the aging time, therefore only the locally equilibrated regime $\omega t \gg 1$ is explored. Only seldom the "slow" regime $\omega t \sim 1$, where FDT violations are expected, is measured. Very recently, direct measurements of FDT violations in the time domain have been reported for insulating spin glasses [340. FDT violations have been measured beyond the quasistationary regime and experimental FD plots have been found to be consistent with results obtained by numerical simulations in spin glass models (see section 77.2.1). However the value of the effective temperature obtained in these experiments is much larger than the annealing temperature at which the system is equilibrated before the 


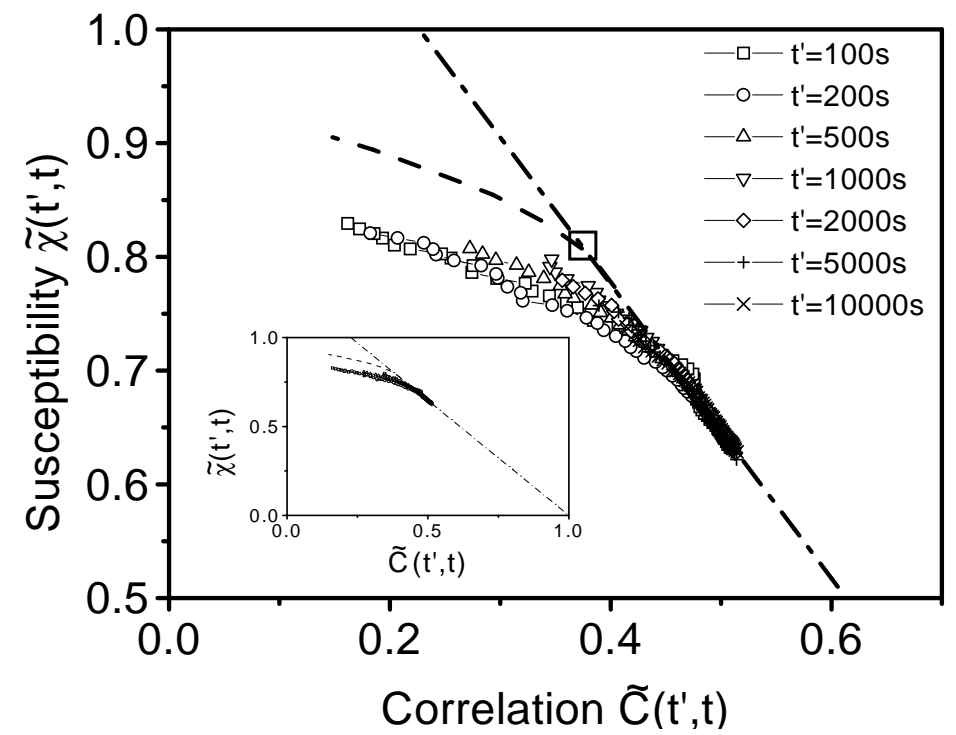

Figure 37. FD plot obtained by measuring voltage autocorrelations and relaxation susceptibility in the insulating spin glass $C d C r_{1.7} I n_{0.3} S_{4}$ quenched from $T=1.2 T_{\mathrm{g}}$ down to $T=0.8 T_{\mathrm{g}}\left(T_{\mathrm{g}}=16.2 K\right)$. Effective temperatures are around $30 K$ and much larger than the annealing temperature 19.4K. From [340].

quenching takes place, casting doubts on the meaning of the effective temperature as a thermodynamic temperature in these experiments. Moreover these measurements reveal that, within the experimentally accessible time window, FD curves $\chi(C)$ (103) are time-dependent and quite far from the expected asymptotic curve. This may explain previously reported discrepancies among FD plots obtained by analyzing the magnetization data of several spin glass systems 341]. These indirect measurements of FDT violations do not show any clear evidence of a universal curve $\chi(C)$ and suggest that experimental measurements are quite far from the asymptotic regime. Experimental FD plots are shown in figure 37

In structural glasses recent measurements show also the existence of FDT violations, however the physical interpretation of these experiments is still unclear. Voltage noise measurements 342 in an electric resonant circuit formed by a capacitor containing glycerol and an inductance show that the Nyquist formula (278) is violated depending on the waiting time and the frequency. By defining the effective temperature $T_{\text {eff }}\left(\omega, t_{w}\right)$ as in (133) (i.e. the temperature that satisfies the Nyquist formula (278) ) experiments reveal that the capacitance ages and FDT violations appear also in the range $\omega t_{w} \gg 1$. This seems to be in contradiction with previous old experimental results in spin glasses. The origin of this discrepancy is presently unclear. Other recent experiments by Ciliberto and coworkers 343, 344] on Laponite, a synthetic clay of charged particles that have the shape of a disc, show strong FD violations. If let evolve inside water, Laponite generates a colloid glass consisting of a packed irregular structure of disks due to the complex pattern of quadrupolar interactions. It is found that the value of the effective temperature $T_{\text {eff }}\left(\omega, t_{w}\right)$, for low enough frequencies, is up to 3 or 4 orders of magnitude larger than the bath temperature. Although this has been interpreted as an evidence for coarsening dynamics (where $T_{\text {eff }} \rightarrow \infty$ ) a conclusive explanation of the origin of these high values 
is still unknown. One interesting aspect of these experiments is the scaling behavior $\omega t^{1 / 2}$ observed in the power spectrum indicative that FDT violations persist even in the regime $\omega t_{w} \gg 1$, in agreement with the previous results on glycerol. Contrarily to what would be expected, noise measurements in a rheological experiment for Laponite do not detect significant FDT violations.

Experiments that successfully clearly demonstrate the existence of effective temperatures related to FDT violations are certainly needed. These preliminary account of results shows that still much work has to be done in aging, driven or granular systems to provide a safe ground to many of these ideas.

\section{Conclusions}

In this review we have presented an overview on what is now an active area of research, i.e. the study of FDT violations in glassy systems. Glassy systems are widespread in nature and found in many different areas covering physics, chemistry or biology. These are non-equilibrium systems which are either in a non-stationary slowly relaxing aging state or in a weakly driven stationary state. Equilibrium systems are often described by a set of intensive parameters such as temperature, pressure or density. In a similar way, in glassy systems an important role is played by the waiting time (aging systems) or the intensity of the driving force and/or its frequency (driven systems). These parameters describe how far from equilibrium the system is. In fact, it is becoming steadily clear that a thermodynamic description of glassy systems can be partially rescued. In this description the glassy regime can be rationalized by using some of the concepts of equilibrium statistical physics such as the existence of a modified version of the FDT, the so called quasi-FDT (QFDT).

Associated to the existence of the QFDT there is the concept of effective temperature. Rather than being a useful parameter to describe the behavior of nonequilibrium systems, the effective temperature has a deeper physical meaning. It could be the vestige of the existence of some dynamical measure underlying the nonequilibrium regime. However we do not know how to prove the existence of the QFDT from first principles in the same way we do not know how to prove Boltzmann equal probability hypothesis in equilibrium theory. Therefore, establishing the existence of this dynamical measure is somehow equivalent to assuming the existence of a QFDT. Then the crucial point is which are the specific predictions, that can be experimentally tested, one can derive from the existence of a QFDT. The measurement of the effective temperature itself appears as the most direct way of challenging the QFDT. However, direct measurements of this non-equilibrium temperature appear quite difficult, results are still very preliminary and much progress is yet to be done to reach a convincing and clear evidence.

The study of several families of models, as described in this review, appears as a very fruitful source of inspiration for new concepts and ideas that could be eventually exported to different classes of problems. However this path is not free of challenges and ambiguities. Indeed what are natural concepts for some families of models appear to be quite artificial in other families. As an example, key concepts in a thermodynamic formulation of the glassy state are the existence of an effective temperature associated to the configurational entropy or complexity, in the same way the bath temperature is associated to the Boltzmann entropy in equilibrium theory. However, what appears to be an interesting quantity describing glassy systems with two-timescales cannot be easily translated into systems with many timescales. Even more, what appears to be a 
meaningful quantity for models having a complex thermodynamics (such as spin-glass models) appears to be meaningless in models described by a trivial Hamiltonian (such as kinetically constrained models).

From the point of view of theoretical studies, our understanding of the existence of a QFDT appears also quite problematic. Most of the solutions we have described in this review are only valid in the case of mean-field interactions, however their validity beyond that limit remains speculative. The use of numerical simulations has aided to bridge the gap. It is quite interesting that most of the results predicted in mean-field models are qualitatively also observed in short-range systems. This tendency to rationalize the behavior of real systems within a mean-field scenario, i.e. far beyond their natural domain of applicability, has become quite standard in the study of glassy systems. The description of the equilibrium properties of spin-glass systems has for many years followed a similar route. Although the knowledge we can gain from numerical simulations in glassy systems is always quite qualitative (either due to the limited range of timescales or to the inherent simplicity of the simulated model) the accumulated evidence, as reported along this review, points toward the emergence of a QFDT in the non-equilibrium regime of glassy systems, reminiscent of how the Boltzmann measure emerges in equilibrium systems. The same conclusion holds for the Edwards measure in granular media.

What will be the future in the research of FDT violations? Although modeling promises to offer new ideas and will clarify our understanding we feel that more progress is certainly needed in basic theory and experiment. In theory we need to understand the origin of the existence of a QFDT right from the microscopics. Cooperative processes in glasses involve a few tens of atoms and occur along nanometric length-scales. How to link the microscopic activated processes to the emergence of macroscopic properties (such as the effective temperature) is a real challenge. In this context, it appears quite interesting to pursue the investigation of the so called fluctuation theorems recently proposed to quantify transient violations of the second law of thermodynamics. From the experimental side the current accumulated knowledge is still too poor and more experiments are certainly needed for this field of research to grow. A future line of progress is the use of nanotechnology devices to do noise measurements over spatially localized regions of nanometric sizes. These devices could be used as a microscope to measure activated processes occurring in small length scales.

Certainly we will see upcoming developments in this exciting area of research. A continuous exchange of ideas among theory, simulation and experiments is highly desirable and certainly needed to improve our current understanding in this field.

Acknowledgments: We are grateful to all our collaborators, much of whose work has been described in this review, including L. L. Bonilla, J. P. Bouchaud, S. Ciuchi, B. Coluzzi, L. F. Cugliandolo, K. Dawson, S. Franz, A. Garriga, J. Hertz, H. Horner, J. Kurchan, L. Leuzzi, E. Marinari, U. Marini Bettolo, M. Mezard, Th. M. Nieuwenhuizen, F. G. Padilla, I. Pagonabarraga, G. Parisi, M. Picco, A. Puglisi, F. Ricci-Tersenghi, F. Rao, M. Rubí, A. Rocco, G. Ruocco, M. Sales, D. Sherrington, F. Sciortino, M. Sellitto, P. Sollich, H.-J. Sommers, H. Sompolinsky and P. Tartaglia. We are also indebted to many other colleagues for enlightening discussions on many of the topics covered in this review. We are particularly grateful to P. Sollich for useful comments and improvement suggestions. FR has been supported by the Spanish Ministerio de Ciencia y Tecnología Grant BFM2001-3525, Generalitat de Catalunya and Acciones-Integradas Italia-España (HI2000-0087). 
Violation of FDT in glassy systems

\section{List of abbreviations}

1RSB One-step Replica Symmetry Breaking

BG Backgammon

BTM Bouchaud Trap Model

EA Edwards-Anderson model

FD Fluctuation-Dissipation

FDR Fluctuation-Dissipation Ratio

FDT Fluctuation-Dissipation Theorem

FILG Frustrated Ising Lattice Gas

IRF Integrated Response Function

IS Inherent Structure

LJ Lennard-Jones

ME Master Equation

MF Mean Field

MCT Mode-Coupling Theory

OSC Oscillator

QFDT Quasi-Fluctuation-Dissipation Theorem

REM Random Energy Model

RFIM Random Field Ising Model

ROM Random Orthogonal Model

RSB Replica Symmetry Breaking

SK Sherrington-Kirkpatrick

TAP Thouless-Anderson-Palmer

$\boldsymbol{T}_{\mathrm{c}}$ Mode-Coupling critical temperature, Dynamical critical temperature

$\boldsymbol{T}_{\text {eff }}$ Effective temperature

$\boldsymbol{T}_{\mathrm{g}}$ Glass transition temperature

$\boldsymbol{T}_{\mathrm{K}}$ Kauzmann temperature

$\boldsymbol{T}_{\mathrm{RSB}}$ Static transition temperature in replica calculations

TRM Thermoremanent Magnetization

TTI Time Translation Invariance

ZFC Zero Field Cooled

\section{References}

[1] L. C. E Struik. Physical Aging in Amorphous Polymers and Other Materials. Elsevier, 1978.

[2] A. Q. Tool. Relation between inelastic deformability and thermal expansion of glass in its annealing range. Acta Mechanica., 29:240-253, 1946.

[3] L. F. Cugliandolo. Dynamics of glassy systems. Preprint, cond-mat/0210312

[4] J. Jackle. Models of the glass transition. Reports on Progres in Physics, 49:171-231, 1986.

[5] C. A. Angell. The glass transition: an assessment of current thinking. Nuclear Physics B, 5A:69-80, 1988.

[6] M. D. Ediger, C. A. Angell and S. R. Nagel. Supercooled liquids and glasses. Journal of Physical Chemistry, 100:13200 -13212, 1996. 
[7] P. G. De Benedetti. Metastable Liquids : concepts and principles. Princeton University Press, 1996.

[8] P. G. Wolynes. Entropy crises in glasses and random heteropolymers. Journal of Research of the National Institute of Standards and Technology, 102:187-194, 1997.

[9] E. A. Di Marzio. The entropy theory of glass formation after 40 years. Computational Materials Science, 4:317-324, 1995.

[10] W. Gotze and L. Sjogren. Relaxation processes in supercooled liquids. Reports on Progres in Physics, 55:241-376, 1992.

[11] W. Gotze. Recent tests of the mode-coupling theory for glassy dynamics. Journal of PhysicsCondensed Matter, 11:A1-A45, 1999.

[12] W. Kob. Computer simulations of supercooled liquids and glasses. Journal of PhysicsCondensed Matter, 11:R85, 1999.

[13] A. P. Young (Ed). Spin Glasses and Random Fields. World Scientific, Singapore, 1998.

[14] J.-P. Bouchaud, L. F. Cugliandolo, J. Kurchan and M. Mezard. Mode-coupling approximations, glass theory and disordered systems. Physica A, 226:243-73, 1996.

[15] F. Ritort and P. Sollich. Glassy dynamics of kinetically constrained models. Preprint, cond-mat/0210382

[16] J.-P. Bouchaud. Granular media: some ideas from statistical physics. Preprint, cond-mat/0211196

[17] Proceedings of the sitges conference, 1996: Complex behaviour of glassy systems. SpringerVerlag, 1997.

[18] Proceedings of the trieste workshop unifying concepts in glassy physiscs. Journal of PhysicsCondensed Matter, 12, 1999.

[19] P. Sollich and F. Ritort (Eds.). Proceedings of the barcelona workshop on glassy dynamics in kinetically constrained models. Journal of Physics-Condensed Matter, 14, 2002.

[20] S.-K. Ma. Statistical Mechanics. World Scientific, 1985.

[21] H. B. Callen. Thermodynamics and introduction to thermostatistics. John Wiley and Sons, 1985.

[22] C. Beck and F. Schloegel. Thermodynamics of Chaotic Systems: an Introduction. Cambridge Nonlinear Science Series. Cambridge University Press, 1993.

[23] L. Onsager. Reciprocal relations in irreversible processes. i. Physical Review, 37:405 - 426, 1931.

[24] L. Onsager. Reciprocal relations in irreversible processes. ii. Physical Review, 38:2265 - 2279, 1931.

[25] M. Plischke and B. Bergensen. Equilibrium statistical physics. World Scientific, 1994.

[26] H. Risken. The Fokker-Planck Equation: Methods of Solution and Applications. Springer, 1996.

[27] G. Parisi. Statistical Field Theory. Frontiers in Physics. Addison-Wesley, 1998.

[28] J. Zinn-Justin. Quantum Field Theory and Critical Phenomena. Internationa Series of Monographs on Physics, n 92. Claredon Press, 1996.

[29] F. R. Gantmacher. The Theory of Matrices. Chelsea, 1959.

[30] R. S. Miller and R. A. MacPhail. Ultraslow nonequilibrium dynamics in supercooled glycerol by stimulated brillouin gain spectroscopy. Journal of Chemical Physics, 106:3393-3401, 1997.

[31] F. G. Padilla and F. Ritort. Langevin dynamics of the lebowitz - percus model. Journal of Physics A, 30:7089-7114, 1997.

[32] H. Horner. Dynamic mean field theory of the sk-spin glass. i. regularization by bond dynamics. Zeitschrift für Physik B, 57:29-37, 1984.

[33] L. F. Cugliandolo and J. Kurchan. Weak ergodicity breaking in mean-field spin-glass models. Philosophical Magazine B, 71:501-14, 1995.

[34] S. Franz and M. Mezard. On mean-field glassy dynamics out of equilibrium. Physica A, 210:48-72, 1994.

[35] M. Mezard, G. Parisi and M. A. Virasoro. Spin-Glass Theory and Beyond. World Scientific (Singapore), 1987.

[36] A. Crisanti and F. Ritort. Inherent structures, configurational entropy and slow glassy dynamics. Journal of Physics-Condensed Matter, 14:1381-1395, 2002.

[37] R. G. Palmer. Broken ergodicity. Advances in Physics, 31:669-735, 1982.

[38] S. A. Brawer. Theory of relaxation in viscous liquids and glasses. Journal of Chemical Physics, 81:954-975, 1984.

[39] J. C. Dyre. Master-equation appoach to the glass transition. Physical Review Letters, 58:792$795,1987$. 
[40] J.-P. Bouchaud. Weak ergodicity breaking and aging in disordered systems. Journal de Physique I (France), 2:1705-13, 1992.

[41] A. Coniglio and M. Nicodemi. A statistical mechanics approach to the inherent states of granular media. Physica A, 296:451-459, 2001.

[42] T. R. Kirkpatrick and P. G. Wolynes. Stable and metastable states in mean-field potts and structural glasses. Physical Review B, 36:8552-8564, 1987.

[43] T. R. Kirkpatrick and D. Thirumalai. Dynamics of the structural glass transition and the p-spin-interaction spin-glass model. Physical Review Letters, 58:2091-2094, 1987.

[44] T. R. Kirkpatrick and D. Thirumalai. p-spin-interaction spin-glass models: Connections with the structural glass problem. Physical Review B, 36:5388-5397, 1987.

[45] W. Kauzmann. Chem. Rev., 43:219, 1948.

[46] S. Franz and M. A. Virasoro. Quasi equilibirum interpretation of aging dynamics. Journal of Physics A, 33:891-905, 2000.

[47] G. Biroli and J. Kurchan. Metastable states in glassy systems. Physical Review E, 64:016101, 2001.

[48] M. Mezard, G. Parisi and M. A. Virasoro. Random free energies in spin glasses. J. Physique Lett., 46:L217-22, 1985.

[49] Th. M. Nieuwenhuizen. Thermodynamic description of a dynamical glassy transition. Journal of Physics A, 31:L201-L207, 1998.

[50] A. Crisanti and F. Ritort. A glass transition scenario based on heterogeneities and entropy barriers. Philosophical Magazine B, 82:143 - 149, 2002.

[51] A. Crisanti and F. Ritort. A real-space description of the glass transition based on heterogeneities and entropy barriers. Preprint, cond-mat/0102104

[52] L. F. Cugliandolo and J. Kurchan. Analytical solution of the off-equilibrium dynamics of a long-range spin-glass model. Physical Review Letters, 71:173-6, 1993.

[53] L. F. Cugliandolo and J. Kurchan. On the out-of-equilibrium relaxation of the sherringtonkirkpatrick model. Journal of Physics A, 27:5749-72, 1994.

[54] S. M. Fielding and P. Sollich. Observable dependence of fluctuation-dissipation relations and effective temperatures. Physical Review Letters, 88:050603, 2002.

[55] P. Sollich, S. Fielding and P. Mayer. Fluctuation-dissipation relations and effective temperatures in simple non-mean field systems. Journal of Physics-Condensed Matter, 14:1683-96, 2002.

[56] M. Goldstein. Viscous liquids and the glass transition: a potential energy barrier picture. Journal of Physical Chemistry, 51:3728-3739, 1969.

[57] F. H. Stillinger and T. A. Weber. Hidden structure in liquids. Physical Review A, 25:978-989, 1982.

[58] F. H. Stillinger and T. A. Weber. Packing structures and transitions in liquids and solids. Science, 225:983-989, 1984.

[59] F. H. Stillinger. Statistical-mechanics of metastable matter - superheated and stretched liquids. Physical Review E, 52:4685-4690, 1995.

[60] A. Heuer. Properties of a glass-forming system as derived from its potential energy landscape. Physical Review Letters, 78:4051-4054, 1997.

[61] S. Buchner and A. Heuer. Potential energy landscape of a model glass former: Thermodynamics, anharmonicities, and finite size effects. Physical Review E, 60:6507-6518, 1999.

[62] S. Sastry, P. G. Debenedetti, F. H. Stillinger, T. B. Schroder, J. C. Dyre and S. C. Glotzer. Potential energy landscape signatures of slow dynamics in glass forming liquids. Physica A, 270:301-8, 1999.

[63] T. B. Schroder, S. Sastry, J. C. Dyre and S. C. Glotzer. Crossover to potential energy landscape dominated dynamics in a model glass-forming liquid. Journal of Chemical Physics, 112:9834-9840, 2000.

[64] L. Angelani, R. Di Leonardo, G. Ruocco, A. Scala and F. Sciortino. Saddles in the energy landscape probed by supercooled liquids. Physical Review Letters, 85:5356-5359, 2000.

[65] A. Crisanti and F. Ritort. Potential energy landscape of finite-size mean-field models for glasses. Europhysics Letters, 51:147, 2000.

[66] A. Crisanti and F. Ritort. Activated processes and inherent structure dynamics of finite-size mean-field models for glasses. Europhysics Letters, 52:640, 2000.

[67] A. Crisanti and F. Ritort. Equilibrium and ageing dynamics of simple models for glasses. Journal of Physics-Condensed Matter, 12:6413-6422, 2000.

[68] A. Crisanti and F. Ritort. Configurational entropy and the one-step rsb scenario in glasses. In A.C.C. Coolen L.P.Hughston P. Sollich and R.F. Streater, editors, Disordered and Complex 
Systems, volume 553. AIP Conference Proceedings, 2001.

[69] G. Biroli and R. Monasson. From inherent structures to pure states: some simple remarks and examples. Europhysics Letters, 50:155-161, 2000.

[70] A. Crisanti, F. Ritort, A. Rocco and M. Sellitto. Inherent structures and nonequilibrium dynamics of one- dimensional constrained kinetic models: a comparison study. Journal of Chemical Physics, 113:10615-10634, 2000.

[71] D. J. Thouless, P. W. Anderson and R. G. Palmer. Solution of 'solvable model of a spin glass'. Philos. Mag., 35:593-601, 1977.

[72] A. J. Bray and M. A. Moore. Metastable states in spin glasses. Journal of Physics C, 13:L469L476, 1980.

[73] A. Crisanti and H.-J. Sommers. Thouless-anderson-palmer approach to the spherical $p$-spin spin glass model. Journal de Physique (France), 5:805-813, 1995.

[74] G. Parisi and M. Potters. Mean-field equations for spin models with orthogonal interaction matrices. Journal of Physics A, 28:5267-85, 1995.

[75] G. Parisi and M. Potters. On the number of metastable states in spin glasses. Europhysics Letters, 32:13-17, 1995.

[76] A. Cavagna, I. Giardina and G. Parisi. Stationary points of the thouless-anderson-palmer free energy. Physical Review B, 57:11251-7, 1998.

[77] R. Monasson. Structural glass transition and the entropy of the metastable states. Physical Review Letters, 75:2847-2850, 1995.

[78] M. Mezard. How to compute the thermodynamics of a glass using a cloned liquid. Physica A, 265:352, 1999.

[79] M. Mezard and G. Parisi. Thermodynamics of glasses: A first principles computation. Physical Review Letters, 82:747-750, 1999.

[80] M. Mezard and G. Parisi. A first-principle computation of the thermodynamics of glasses. Journal of Chemical Physics, 111:1076-1095, 1999.

[81] B. Coluzzi, G. Parisi and P. Verrocchio. Thermodynamical liquid-glass transition in a lennardjones binary mixture. Physical Review Letters, 84:306-309, 2000.

[82] B. Coluzzi, G. Parisi and P. Verrocchio. Lennard-jones binary mixture: A thermodynamical approach to glass transition. Journal of Chemical Physics, 112:2933, 2000.

[83] E. De Santis, G. Parisi and F. Ritort. On the static and dynamical transition in the mean-field potts glass. Journal of Physics A, 28:3025-3041, 1995.

[84] F. Ritort. Classical and quantum behavior in mean-field glassy systems. In C. J. Perez-Vicente and M. Rubi, editors, Complex behavior in glassy systems. Springer Verlag, 1996.

[85] S. Franz and G. Parisi. Recipes for metastable states in spin glasses. Journal de Physique I (France), 5:1401-15, 1995.

[86] M. Cardenas, S. Franz and G. Parisi. Glass transition and effective potential in the hypernetted chain approximation. Journal of Physics A, 31:L163-L169, 1998.

[87] M. Cardenas, S. Franz and G. Parisi. Constrained boltzmann-gibbs measures and effective potential for glasses in hypernetted chain approximation and numerical simulations. Journal of Chemical Physics, 110:1726-34, 1999.

[88] R. J. Speedy. The entropy of a glass. Molecular Physics, 80:1105-1120, 1993.

[89] R. J. Speedy. Estimates of the configurational entropy of a liquid. Journal of Physical Chemistry B, 105:11737 -11742, 2001.

[90] R. J. Speedy. Configurational entropy and diffusion in a hard disc fluid. Journal of Chemical Physics, 114:9069-9074, 2001.

[91] F. Sciortino, W. Kob and P. Tartaglia. Inherent structure entropy of supercooled liquids. Physical Review Letters, 83:3214-3217, 1999.

[92] S. Mossa, E. La Nave, H. E. Stanley, C. Donati, F. Sciortino and P. Tartaglia. Dynamics and configurational entropy in the lewis-wahnstrm model for supercooled orthoterphenyl. Physical Review E, 65:041205, 2002.

[93] A. Crisanti and F. Ritort. unpublished, 2002.

[94] S. Sastry. The relationship between fragility, configurational entropy and the potential energy landscape of glass-forming liquids. Nature, 409:164-167, 1999.

[95] B. Coluzzi, E. Marinari, G. Parisi and H. Rieger. On the energy minima of the sherringtonkirkpatrick model. Journal of Physics A, 33:3851-62, 2000.

[96] B. Coluzzi, A. Crisanti, E. Marinari, F. Ritort and A. Rocco. A new method to compute the configurational entropy in spin glasses. Preprint, cond-mat/0105391

[97] P. Hohenberg and B. Shraiman. Chaotic behavior of an extended system. Physica D, 37:109$15,1989$.

[98] I. M. Hodge. Macromolecules, 20:2897, 1987. 
[99] I. M. Hodge. Enthalpy relaxation and recovery in amorphous materials. Journal of NonCrystalline Solids, 169:211-66, 1994.

[100] L. F. Cugliandolo, J. Kurchan and L. Peliti. Energy flow, partial equilibration, and effective temperatures in systems with slow dynamics. Physical Review E, 55:3898-914, 1997.

[101] L. F. Cugliandolo and J. Kurchan. Thermal properties of slow dynamics. Physica A, 263:242$51,1999$.

[102] L. F. Cugliandolo and J. Kurchan. Thermal properties of slow dynamics. Physica A, 263:242251, 1999.

[103] A. Garriga and F. Ritort. Validity of the zero-thermodynamic law in off-equilibrium coupled harmonic oscillators. European Physical Journal B, 20:105-122, 2001.

[104] L. F. Cugliandolo, D. S. Dean and J. Kurchan. Fluctuation-dissipation theorems and entropy production in relaxational systems. Physical Review Letters, 79:2168-71, 1997.

[105] A. Garriga and F. Ritort. Heat transfer and fourier's law in off-equilibrium systems. European Physical Journal B, 21:115-120, 2001.

[106] R. Exartier and L. Peliti. Measuring effective temperatures in out-of-equilibrium driven systems. European Physical Journal B, 16:119-26, 2000.

[107] Th. M. Nieuwenhuizen. Thermodynamics of the glassy state: effective temperature as an additional parameter. Physical Review Letters, 80:5580-5583, 1998.

[108] Th. M. Nieuwenhuizen. Thermodynamic picture of the glassy state gained from exactly solvable models. Physical Review E, 61:267-292, 2000.

[109] Th. M. Nieuwenhuizen. Ehrenfest relations at the glass transition: solution to an old paradox. Physical Review Letters, 79:1317-1320, 1997.

[110] S. F. Edwards. Theory of powders. Physica A, 157:1080-90, 1898.

[111] S. F. Edwards and R. B. S. Oakeshott. Theory of powders. Physica A, 157:1080-90, 1989.

[112] A. Mehta and S. F. Edwards. Statistical mechanics of powder mixtures. Physica A, 157:1091$100,1989$.

[113] S. F. Edwards. The flow of powders and of liquids of high viscosity. Journal of PhysicsCondensed Matter, 2:63-9, 1990.

[114] A. Mehta and S. F. Edwards. A phenomenological approach to relaxation in powders. Physica A, 168:714-22, 1990.

[115] R. Monasson and O. Pouliquen. Entropy of particle packings: an illustration on a toy model. Physica A, 236:395-410, 1997.

[116] J. Berg, S. Franz and M. Sellitto. Testing the edwards hypothesis in spin systems under tapping dynamics. European Physical Journal B, 26:349, 2002.

[117] L. Berthier, J.-L. Barrat and J. Kurchan. Dynamic ultrametricity in spin glasses. Physical Review E, 63:016105, 2001.

[118] A. Coniglio, A. Fierro and M. Nicodemi. Applications of the statistical mechanics of inherent states to granular media. Physica A, 302:193-201, 2001.

[119] J. Kurchan. Emergence of macroscopic temperatures in systems that are not thermodynamical macroscopically: towards a thermodynamical description of slow granular rheology. Journal of Physics-Condensed Matter, 12:6611-17, 2002.

[120] T. R. Kirkpatrick and P. G. Wolynes. Connections between some kinetic and equilibrium theories of the glass transition. Physical Review A, 35:3072-3080, 1987.

[121] E. Leutheusser. Dynamical model of the liquid-glass transition. Physical Review A, 29:2765$73,1984$.

[122] U. Bengtzelius, W. Goetze and A. Sjolander. Dynamics of supercooled liquids and the glass transition. Journal of Physics C, 17:5915-34, 1984.

[123] W. Goetze. Aspect of structural glass transitions. In D. Levesque J. P. Hansen and J. ZinnJustin, editors, Liquids, Freezing and Glass Transition. North-Holland, Amsterdam, 1991.

[124] D. J. Gross, I. Kanter and H. Sopolinsky. Mean-field theory of the potts glass. Physical Review Letters, 55:304307, 1985.

[125] P. Goldbardt and D. Sherrington. Replica theory of the uniaxial quadrupolar glass. Journal of Physics C, 18:1923-1940, 1985.

[126] B. Derrida. Random-energy model: An exactly solvable model of disordered systems. Physical Review B, page 26132626, 1981.

[127] D. J. Gross and M. Mezard. The simplest spin glass. Nuclear Physics B, 240:431-52, 1984.

[128] E. Gardner. Spin glasses with p-spin interactions. Nuclear Physics B, pages 747-65, 1985.

[129] D. Sherrington and A. Kirkpatrick. Solvable model of a spin-glass. Physical Review Letters, 35:17921796, 1975.

[130] S. Kirkpatrick and D. Sherrington. Infinite-ranged models of spin-glasses. Physical Review B, 17:43844403, 1978. 
[131] A. Crisanti and H.-J. Sommers. The spherical p-spin interaction spin glass model: The statics. Zeitschrift für Physik B, 87:341-354, 1992.

[132] A. Crisanti, H. Horner and H.-J. Sommers. The spherical $p$-spin interaction spin glass model: The dynamics. Zeitschrift für Physik B, 92:257-271, 1993.

[133] P. C. Hohenberg and B. I. Halperin. Theory of dynamic critical phenomena. Review of Modern Physics, 49:435-79, 1977.

[134] H. Sompolinsky and A. Zippelius. Relaxational dynamics of the edwards-anderson model and the mean-field theory of spin-glasses. Physical Review B, 25:6860-75, 1982.

[135] G. Parisi. Order parameter for spin-glasses. Physical Review Letters, 50:19461948, 1983.

[136] J. Kurchan, G. Parisi and M. A. Virasoro. Barriers and metastable states as saddle points in the replica approach. Journal de Physique I (France), 3:1819-38, 1993.

[137] S. Franz and G. Parisi. Phase diagram of coupled glassy systems: A mean-field study. Physical Review Letters, 79:2486-2489, 1997.

[138] A. Cavagna, I. Giardina and G. Parisi. An investigation of the hidden structure of states in a mean-field spin-glass model. Journal of Physics A, 30:7021-7038, 1997.

[139] A. Barrat, S. Franz and G. Parisi. Temperature evolution and bifurcations of metastable states in mean-field spin glasses, with connections with structural glasses. Journal of Physics A, 30:5593-5612, 1997.

[140] A. Cavagna, I. Giardina and G. Parisi. Structure of metastable states in spin glasses by means of a three replica potential. Journal of Physics A, 30:4449-4466, 1997.

[141] H. Sompolinsky. Time-dependent order parameters in spin-glasses. Physical Review Letters, 47:935938, 1981.

[142] H. Horner. Dynamic mean-field theory of a slowly cooled spin glass. Europhysics Letters, 2:487-91, 1986.

[143] L. B. Ioffe. Quasiequilibrium states of spin glasses. Physical Review B, 38:51815183, 1988.

[144] L. F. Cugliandolo and P. Le Doussal. Large time nonequilibrium dynamics of a particle in a random potential. Physical Review E, 53:1525-52, 1996.

[145] Th. M. Nieuwenhuizen. Exactly solvable model of a quantum spin glass. Physical Review Letters, 74:4289-4292, 1995.

[146] J. A. Hertz, D. Sherrington and Th. M. Nieuwenhuizen. Competition between glassiness and order in a multispin glass. Physical Review E, 60:R2460-R2463, 1999.

[147] S. Ciuchi and A. Crisanti. Different scenarios for critical glassy dynamics. Europhysics Letters, 49:754-760, 2000.

[148] A. Crisanti, J. Hertz, Th. M. Nieuwenhuizen and D. Sherrington. Static and dynamical direct and tap approaches to the p-spin model with a ferromagnetic bias. unpublished, 2003.

[149] S. Ciuchi and F. De Pasquale. Nonlinear relaxation and ergodicity breakdown in random anisotropy spin glasses. Nuclear Physics B, 300(FS22):31-60, 1988.

[150] L. F. Cugliandolo and D. S. Dean. ull dynamical solution for a spherical spin-glass model. Journal of Physics A, 28:4213-4234, 1995.

[151] L. F. Cugliandolo and D. S. Dean. Full dynamical solution for a spherical spin-glass model. Journal of Physics A, 28:4213-4234, 1995.

[152] M. Campellone, G. Parisi and P. Ranieri. Dynamical fluctuations in an exactly solvable model of spin glasses. Journal of Physics A, 31:1893-900, 1998.

[153] W. Zippold, R. Kuhn and H. Horner. Non-equilibrium dynamics of simple spherical spin models. European Physical Journal B, 13:531-537, 2000.

[154] S. Ciuchi, F. De Pasquale and P. Monachesi. Relaxation phenomena in two interacting spin lattices. Journal of Physics-Condensed Matter, 2:4921-4934, 1990.

[155] H. Kinzelbach and H. Horner. Dynamics of manifolds in random media. ii. long range correlations in the disordered medium. Journal de Physique I (France), 3:1901-19, 1993.

[156] H. Sompolinsky and A. Zippelius. Dynamic theory of the spin-glass phase. Physical Review Letters, 47:359362, 1981.

[157] H.-J. Sommers and K. H. Fisher. Dynamic scaling for spin glasses near the de almedia-thouless line. Zeitschrift für Physik B, 58:125-31, 1985.

[158] H.-J. Sommers. On the dynamic mean field theory of spin glasses. Zeitschrift für Physik B, 50:97-105, 1983

[159] A. Baldassarri, L. F. Cugliandolo, J. Kurchan and G. Parisi. On the out-of-equilibrium order parameter in long-range spin-glasses. Journal of Physics A, 28:1831-1845, 1995.

[160] S. Franz, M. Mezard, G. Parisi and L. Peliti. Measuring equilibrium properties in aging systems. Physical Review Letters, 81:1758-1761, 1998.

[161] S. Franz, M. Mezard, G. Parisi and L. Peliti. The response of glassy systems to random perturbations: A bridge between equilibrium and off-equilibrium. Journal of Statistical 
Physics, 97:459-88, 1999.

[162] G. Blatter, M. V. Feigel'man, V. B. Geshkenbein, A. L. Larkin and V. M. Vinokur. Vortices in high-temperature superconductors. Review of Modern Physics, 66:1125-388, 1994.

[163] T. Nattermann and P. Rujan. Random field and other systems dominated by disorder fluctuations. Int. J. Mod. Phys. B, 3:1597-654, 1989.

[164] D. Cule and Y. Shapir. Glassy roughness of a crystalline surface upon a disordered substrate. Physical Review Letters, 74:114117, 1995.

[165] Th. M. Nieuwenhuizen. Solvable glassy system: Static versus dynamical transition. Physical Review Letters, 78:34913494, 1997.

[166] L. F. Cugliandolo, J. Kurchan and P. Le Doussal. Large time out-of-equilibrium dynamics of a manifold in a random potential. Physical Review Letters, 76:2390-3, 1996.

[167] A. Engel. Replica symmetry breaking in zero dimension. Nuclear Physics B, 410:617-46, 1993.

[168] H. Yoshino. Aging effects of an elastic string diffusing in a disordered media. Physical Review Letters, 81:1493-1496, 1998.

[169] E. Pitard and E. I. Shakhnovich. Mode-coupling theory for heteropolymers. Physical Review E, 63:041501/1-14, 2001.

[170] L. F. Cugliandolo, J. Kurchan and G. Parisi. Off equilibrium dynamics and aging in unfrustrated systems. Journal de Physique I (France), 4:1641-56, 1994.

[171] F. Corberi, M. Nicodemi, M. Piccioni and A. Coniglio. Off-equilibrium dynamics in a singular diffusion model. Physical Review Letters, 83:5054-7, 1999.

[172] F. Corberi, M. Nicodemi, M. Piccioni and A. Coniglio. Slow dynamics and aging in a constrained diffusion model. Physical Review E, 63:031106, 2001.

[173] A. Barrat and M. Mezard. Phase space diffusion and low temperature aging. Journal de Physique I (France), 5:941-947, 1995

[174] B. Derrida. Random-energy model: Limit of a family of disordered models. Physical Review Letters, 45:7982, 1980.

[175] B. Derrida. Random-energy model: An exactly solvable model of disordered systems. Physical Review B, 24:26132626, 1981.

[176] C. Monthus and J.-P. Bouchaud. Models of traps and glass phenomenology. Journal of Physics A, 29:3847-3869, 1996

[177] J.-P. Bouchaud, A. Comtet and C. Monthus. On a dynamical model of glasses. Journal de Physique (France), 5:1521-6, 1995.

[178] J.-P. Bouchaud and D. S. Dean. Aging on parisi's tree. Journal de Physique I (France), 5:265, 1995.

[179] E. Bertin and J.-P. Bouchaud. Dynamical ultrametricity in the critical trap model. Journal of Physics A, 35:3039-3051, 2002.

[180] T. Odagaki. Glass transition singularities. Physical Review Letters, 75:3701-3704, 1995.

[181] F. Ritort. Universal dependence of the fluctuation-dissipation ratio on the transition rates in trap models. Preprint, cond-mat/0303445

[182] E. Lippiello and M. Zannetti. Fluctuation dissipation ratio in the one-dimensional kinetic Ising model. Physical Review E, 61:3369-3374, 2000.

[183] P. Sollich, F. Lequeux, P. Hebraud and M. Cates. Rheology of soft glassy materials. Physical Review Letters, 70:2020, 1997.

[184] P. Sollich. Rheological constitutive equation for a model of soft glassy materials. Physical Review E, 58:738-759, 1998.

[185] S. M. Fielding and P. Sollich. Equivalence of driven and ageing fluctuation-dissipation relation in the trap model. Physical Review E, 67:011101, 2003.

[186] J. Kurchan and L. Laloux. Phase space geometry and slow dynamics. Journal of Physics A, 29:1929-48, 1996

[187] L. Leuzzi and F. Ritort. Disordered backgammon model. Physical Review E, 65:56125, 2002.

[188] A. Garriga. The zeroth law in structural glasses: an example. Journal of Physics-Condensed Matter, 14:1581-1588, 2002.

[189] L. L. Bonilla, F. G. Padilla, G. Parisi and F. Ritort. Analytical solution of the monte carlo dynamics of a simple spin-glass model. Europhysics Letters, 34:159-64, 1996.

[190] L. L. Bonilla, F. G. Padilla, G. Parisi and F. Ritort. Closure of the monte carlo dynamical equations in the spherical sherrington-kirkpatrick model. Physical Review B, 54:4170-4182, 1996.

[191] L. L. Bonilla, F. G. Padilla and F. Ritort. Aging in the linear harmonic oscillator. Physica A, 250:315-326, 1998.

[192] L. Leuzzi and Th. M. Nieuwenhuizen. Inherent structures in models for fragile and strong glass. Physical Review E, 64:066125, 2001. 
[193] L. Leuzzi and Th. M. Nieuwenhuizen. Effective temperatures in an exactly solvable model for a fragile glass. Physical Review E, 64:011508, 2001.

[194] L. Leuzzi and Th. M Nieuwenhuizen. Exactly solvable model glass with a facilitated dynamics. Journal of Physics-Condensed Matter, 14:1637, 2002.

[195] F. Ritort. Glassiness in a model without energy barriers. Physical Review Letters, 75:11901193, 1995.

[196] C. Godreche, J.-P. Bouchaud and M. Mezard. Entropy barriers and slow relaxation in some random walk models. Journal of Physics A, 28:L603-L611, 1995.

[197] D. Arora, D. P. Bhatia and M.A. Prasad. Exact solutions of some urn models of relaxation in glassy dynamics. Physical Review E, 60:145-148, 1999.

[198] A. Lipowski. Absorption time in certain urn models. Journal of Physics A, 30:L91-L94, 1997.

[199] S. Franz and F. Ritort. Dynamical solution of a model without energy barriers. Europhysics Letters, 31:507-512, 1995.

[200] S. Franz and F. Ritort. Glassy mean-field dynamics of the backgammon model. Journal of Statistical Physics, 85:131-150, 1996.

[201] C. Godreche and J. M. Luck. Long-time regime and scaling of correlations in a simple model with glassy behaviour. Journal of Physics A, 29:1915-1928, 1996.

[202] S. Franz and F. Ritort. Relaxation processes and entropic traps in the backgammon model. Journal of Physics A, 30:L359-L365, 1997.

[203] A. Prados, J. J. Brey and B. Sanchezrey. Glassy behavior in a simple model with entropy barriers. Physical Review B, 55:6343-6355, 1997.

[204] C. Godreche and J. M. Luck. Correlation and response in the Backgammon model: the Ehrenfest legacy. Journal of Physics A, 32:6033-6054, 1999.

[205] C. Godreche and J. M. Luck. Response of non-equilibrium systems at criticality: ferromagnetic models in dimension two and above. Journal of Physics A, 33:9141-9164, 2000.

[206] A. Garriga, I. Pagonabarraga and F. Ritort. work in progress, 2003.

[207] C. Godreche and J. M. Luck. Nonequilibrium dynamics of the zeta urn model. European Physical Journal B, 23:473-486, 2001.

[208] C. Godreche and J. M. Luck. Nonequilibrium dynamics of urn models. Journal of PhysicsCondensed Matter, 14:1601-15, 2002.

[209] R. J. Glauber. Time-dependent statistics of the ising model. Journal Mathematical Physics, 4:294-307, 1963.

[210] A. J. Bray. Upper and lower bounds on dynamic correlations in the griffiths phase. Journal of Physics A, 22:L81-L85, 1989.

[211] A. Prados, J. J. Brey and B. Sanchezrey. Aging in the one-dimensional Ising model with Glauber dynamics. Europhysics Letters, 40:13-18, 1997.

[212] C. Godreche and J. M. Luck. Response of non-equilibrium systems at criticality: exact results for the Glauber-Ising chain. Journal of Physics A, 33:1151-1170, 2000.

[213] C. Godreche and J. M. Luck. Nonequilibrium critical dynamics of ferromagnetic spin systems. Journal of Physics-Condensed Matter, 14:1589-99, 2002.

[214] F. Corberi, E. Lippiello and M. Zannetti. Slow relaxation in the large-n model for phase ordering. Physical Review E, 65:046136-11, 2002.

[215] J. P. Garrahan P. M. Mayer, L. Berthier and P. Sollich. Fluctuation-dissipation relations in the non-equilibrium dynamics of ising models. Preprint, cond-mat/0301493

[216] M. Henkel, M. Pleimling, C. Godreche and J. M. Luck. Aging, phase ordering, and conformal invariance. Physical Review Letters, 87:265701, 2001.

[217] F. Corberi, E. Lippiello and M. Zannetti. Interface fluctuations, bulk fluctuations, and dimensionality in the off-equilibrium response of coarsening systems. Physical Review E, 63:061506, 2001.

[218] P. Calabrese and A. Gambassi. Aging in ferromagnetic systems at criticality near four dimensions. Physical Review E, 65:066120, 2002.

[219] P. Calabrese and A. Gambassi. Two-loop critical fluctuation-dissipation ratio for the relaxational dynamics of the o(n) landau-ginzburg hamiltonian. Physical Review E, 66:066101, 2002.

[220] L. Berthier, P. C. W. Holsworth and M. Sellitto. Nonequilibrium critical dynamics of the two-dimensional xy model. Journal of Physics A, 34:1805-1824, 2001.

[221] B. Bernu, J. P. Hansen, Y. Hiwatari and G. Pastore. Soft-sphere model for the glass transition in binary alloys: Pair structure and self-diffusion. Physical Review A, 36:48914903, 1987.

[222] J.-L. Barrat, J. N. Roux and J. P. Hansen. Diffusion, viscosity and structural slowing down in soft sphere alloys near the kinetic glass transition. Chemical Physics, 149:197-208, 1990.

[223] J. P. Hansen and S. Yip. Molecular dynamics investigations of slow relaxations in supercooled 
liquids. Transp.Theory Stat. Phys., 24:1149-78, 1995.

[224] G. Parisi. Short-time aging in binary glasses. Journal of Physics A, 30:L765-70, 1997.

[225] G. Parisi. Off-equilibrium fluctuation-dissipation relation in fragile glasses. Physical Review Letters, 79:3660-3663, 1997.

[226] G. Parisi. Numerical indications for the existence of a thermodynamic transition in binary glasses. Journal of Physics A, 30:8523-8539, 1997.

[227] R. Di Leonardo, L. Angelani, G. Parisi and G. Ruocco. Off-equilibrium effective temperature in monatomic lennard-jones glass. Physical Review Letters, 84:6054-6057, 2000.

[228] L. Angelani, R. Di Leonardo, G. Parisi and G. Ruocco. Topological description of the aging dynamics in simple glasses. Physical Review Letters, 87:055502/1-4, 2001.

[229] W. Kob and H. C. Andersen. Scaling behavior in the beta -relaxation regime of a supercooled lennard-jones mixture. Physical Review Letters, 73:13761379, 1994.

[230] W. Kob and H. C. Andersen. Testing mode-coupling theory for a supercooled binary lennardjones mixture i: The van hove correlation function. Physical Review E, 51:46264641, 1995.

[231] W. Kob and H. C. Andersen. Testing mode-coupling theory for a supercooled binary lennardjones mixture. ii. intermediate scattering function and dynamic susceptibility. Physical Review E, 52:41344153, 1995.

[232] T. Gleim, W. Kob and K. Binder. ow does the relaxation of a supercooled liquid depend on its microscopic dynamics? Physical Review Letters, 81:44044407, 1998.

[233] J.-L. Barrat and W. Kob. Aging and the fluctuation dissipation ratio in a lennard-jones fluid. Journal of Physics A, 11:A247-A252, 1999.

[234] J.-L. Barrat and W. Kob. Fluctuation-dissipation ratio in an aging lennard-jones glass. Europhysics Letters, 46:637-42, 1999.

[235] W. Kob and J.-L. Barrat. Fluctuations, response and aging dynamics in a simple glass-forming liquid out of equilibrium. European Physical Journal B, 13:319, 2000.

[236] L. Angelani, G. Parisi, G. Ruocco and G. Viliani. Connected network of minima as a model glass:long time dynamics. Physical Review Letters, 81:4648, 1998.

[237] L. Angelani, G. Parisi, G. Ruocco and G. Viliani. Potential energy landscape and long-time dynamics in a simple model glass. Physical Review E, 61:1681-91, 2000.

[238] F. Sciortino and P. Tartaglia. Extension of the fluctuation-dissipation theorem to the physical aging of a model glass-formingliquid. Physical Review Letters, 86:107-110, 2001.

[239] C. Brangian, W. Kob and K. Binder. Finite-size scaling at the dynamical transition of the mean-field 10-state potts glass. Europhysics Letters, 53:756-61, 2001.

[240] C. Brangian, W. Kob and K. Binder. Statics and dynamics of the ten-state mean-field potts glass model: a monte carlo study. Journal of Physics A, 35:191-216, 2002.

[241] E. Marinari, G. Giorgio and F. Ritrot. Replica field theory for deterministic models. ii. a non-random spin glass with glassy behaviour. Journal of Physics A, 27:7647-68, 1994.

[242] A. Barrat and S. Franz. Basins of attraction of metastable states of the spherical p-spin model. Journal of Physics A, 31:L119-L127, 1998.

[243] F. Rao, A. Crisanti and F. Ritort. Frequency-domain study of relaxation in a spin glass model for the structural glass transition. Preprint, cond-mat/xxxxxx.

[244] W. Kob, F. Sciortino and P. Tartaglia. Aging as dynamics in configuration space. Europhysics Letters, 49:590, 2000.

[245] E. Marinari, G. Parisi and J. J. Ruiz-Lorenzo. Numerical simulations of spin glass systems. In A. P. Young, editor, Spin glasses and random fields. World Scientific, 1997.

[246] J-O. Andersson, J. Mattson and P. Svedlindh. Monte carlo studies of ising spin-glass systems: Aging behavior and crossover between equilibrium and nonequilibrium dynamics. Physical Review B, 46:82978304, 1992.

[247] S. Franz and H. Rieger. Fluctuation-dissipation ratio in three-dimensional spin glasses. Journal of Statistical Physics, 79:749-58, 1995.

[248] L. F. Cugliandolo, J. Kurchan and F. Ritort. Evidence of aging in spin-glass mean-field models. Physical Review B, 49:6331-6334, 1994.

[249] E. Marinari, G. Parisi, F. Ricci-Tersenghi and J. J. Ruiz-Lorenzo. Violation of the fluctuationdissipation theorem in finite-dimensional spin glasses. Journal of Physics A, 31:2611-2620, 1998.

[250] G. Parisi, F. Ricci-Tersenghi and J. J. Ruiz-Lorenzo. Generalized off-equilibrium fluctuationdissipation relations in random ising systems. European Physical Journal B, 11:317-325, 1999.

[251] G. Parisi, F. Ricci-Tersenghi and J. J. Ruiz-Lorenzo. Dynamics of the four-dimensional spin glass in a magnetic field. Physical Review B, 57:13617-13623, 1998.

[252] G. Parisi, F. Ricci-Tersenghi and J. J. Ruiz-Lorenzo. Universality in the off-equilibrium critical 
Violation of FDT in glassy systems

dynamics of the three-dimensional diluted ising model. Physical Review E, 60:5198-5201, 1999.

[253] E. Marinari, G. Parisi, F. Ricci-Tersenghi and J. J. Ruiz-Lorenzo. Off-equilibrium dynamics at very low temperatures in three-dimensional spin glasses. Journal of Physics A, 33:23732382, 2000.

[254] M. Picco, F. Ricci-Tersenghi and F. Ritort. Aging effects and dynamic scaling in the 3d edwards-anderson spin glasses: a comparison with experiments. European Physical Journal B, 21:211-217, 2001.

[255] G. Parisi and G. Toulouse. A simple hypothesis for the spin glass phase of the infinite-ranged sk model. J. Physique Lett., 41:L361-4, 1980.

[256] J. Vannimenus, G. Toulouse and G. Parisi. Study of a simple hypothesis for the mean-field theory of spin-glasses. Journal de Physique (France), 42:565-71, 1981.

[257] D. Alvarez, S. Franz and F. Ritort. Fragile-glass behavior of a short-range p-spin model. Physical Review B, 54:9756-9764, 1996.

[258] E. Marinari, C. Naitza, G. Parisi, M. Picco, F. Ritort and F. Zuliani. A general method to determine replica symmetry breaking transitions. Physical Review Letters, 81:1698-1702, 1998.

[259] S. Franz and G. Parisi. Critical properties of a three dimensional p-spin model. European Physical Journal B, 8:417-422, 1999.

[260] M. Campellone, B. Coluzzi and G. Parisi. Numerical study of a short-range p-spin glass model in three dimensions. Physical Review B, 58:12081-12089, 1998.

[261] G. Parisi, M. Picco and F. Ritort. Continuous phase transition in a model without time-reversal symmetry. Physical Review E, 60:58-68, 1999.

[262] A. Barrat and L. Berthier. Real-space application of the mean-field description of spin-glass dynamics. Physical Review Letters, 87:087204, 2001.

[263] H. E. Castillo, C. Chamon, L. F. Cugliandolo and M. P. Kennett. Heterogeneous aging in spin glasses. Physical Review Letters, 88:237201-4, 2002.

[264] M. Nicodemi and A. Coniglio. The glassy transition of the frustrated ising lattice gas. Journal of Physics A, 30:L187-94, 1997.

[265] D.A. Stariolo and J. J. Arenzon. Off-equilibrium dynamics of the frustrated ising lattice gas. Physical Review E, 59:R4762-R4765, 1999.

[266] J. J. Arenzon, F. Ricci-Tersenghi and D. A. Stariolo. Dynamics of the frustrated ising lattice gas. Physical Review E, 62:5978-5985, 2000.

[267] A. Crisanti and L. Leuzzi. First order phase transition and phase coexistence in a spin-glass model. Physical Review Letters, 89:237204, 2002.

[268] A. J. Bray. Theory of phase ordering kinetics. Advances in Physics, 43:357, 1994.

[269] D. S. Fisher and D. A. Huse. Nonequilibrium dynamics of spin glasses. Physical Review B, 38:373385, 1988

[270] D. S. Fisher. Dynamics and domain walls: Is the landscape paradigm instructive? Physica $D, 107: 204-217,1997$.

[271] D. Kivelson and G. Tarjus. The kauzmann paradox interpreted via the theory of frustrationlimited-domains. Journal of Chemical Physics, 109:5481-5486, 1998.

[272] A. Barrat. Monte-carlo simulations of the violation of the fluctuation-dissipation theorem in domain growth processes. Physical Review E, 57:3629-3632, 1998.

[273] L. Berthier, J.-L. Barrat and J. Kurchan. Response function of coarsening systems. European Physical Journal B, 11:635-641, 1999.

$[274]$ D. A. Stariolo and S. A. Cannas. Violation of the fluctuation-dissipation theorem in a twodimensional ising model with dipolar interactions. Physical Review B, 60:3013-16, 1999.

[275] D. Kivelson, S. A. Kivelson, X. Zhao, Z. Nussinov and T. Gilles. A thermodynamic theory of supercooled liquids. Physica A, 219:27-38, 1995.

[276] L. P. Pryadko, S. Kivelson and D. W. Hone. Instability of charge ordered states in doped antiferromagnets. Physical Review Letters, 80:56515654, 1998.

[277] L. C. Sampaio, M. P. de Albuquerque and F. S. de Menezes. Magnetic relaxation and formation of magnetic domains in ultrathin films with perpendicular anisotropy. Physical Review B, 54:64656472, 1996.

[278] A. B. MacIsaac, J. P. Whitehead, M. C. Robinson and K. De Bell. Striped phases in twodimensional dipolar ferromagnets. Physical Review B, 51:1603316045, 1995.

[279] F. Ricci-Tersenghi and F. Ritort. Absence of ageing in the remanent magnetization in migdalkadanoff spin glasses. Journal of Physics A, pages 3727-3734, 2000.

[280] J. L. Lebowitz and J. K. Percus. Mean spherical model for lattice gases with extended hard cores and continuum fluids. Physical Review, 144:251258, 1966. 
[281] J. M. Kosterlitz, D. J. Thouless and R. C. Jones. Spherical model of a spin-glass. Physical Review Letters, 36:12171220, 1976.

[282] A. Cannas, D. A. Stariolo and F. A. Tamarit. Dynamics of ferromagnetic spherical spin models with power law interactions: exact solution. Physica A, 294:362-74, 2001.

[283] G. Parisi. Asymmetric neural networks and the process of learning. Journal of Physics A, 19:L675-L680, 1986.

[284] A. Crisanti and H. Sompolinsky. Dynamics of spin system with random asymmetric bonds: Langevin dynamics and a spherical model. Physical Review A, 36:4922-4939, 1987.

[285] A. Crisanti and H. Sompolinsky. Dynamics of spin system with random asymmetric bonds: Ising spins and glauber dynamics. Physical Review A, 37:4865-4874, 1988.

[286] L. F. Cugliandolo, J. Kurchan, P. Le Doussal and L. Peliti. Glassy behaviour in disordered systems with nonrelaxational dynamics. Physical Review Letters, 78:350-3, 1997.

[287] L. Berthier, J.-L. Barrat and J. Kurchan. A two-time-scale, two-temperature scenario for nonlinear rheology. Physical Review E, 61:5464-5472, 2000.

[288] L. Berthier and J.-L. Barrat. Shearing a glassy material: Numerical tests of non-equilibirum mode-coupling approaches and experimental proposals. Physical Review Letters, 89:095702, 2002.

[289] L. Berthier and J.-L. Barrat. Non-equilibrium dynamics and fluctuation-dissipation relation in a sheared fluid. Journal of Chemical Physics, 116:6228-42, 2002.

[290] J.-L. Barrat and L. Berthier. Fluctuation-dissipation relation in a sheared fluid. Physical Review E, 63:012503, 2001.

[291] L. Angelani, G. Ruocco, F. Sciortino, P. Tartaglia and F. Zamponi. Crossover between equilibrium and shear-controlled dynamics in sheared liquids. Physical Review E, 66:061505, 2002.

[292] R. G. Larson. The structure and Rheology of complex fluids. Oxford University Press, New York, 1999

[293] D. Bedeaux and J. M. Rubi. Nonequilibrium thermodynamics of colloids. Physica A, 305:360$370,2002$.

[294] D. Jou, J. Casas-Vazquez and G. Lebon. Extended irreversible thermodynamics revisited (1988-98). Reports on Progres in Physics, 62:1035-1142, 1999.

[295] S. R. de Groot and P. Mazur. Non-Equilibrium Thermodynamics. Dover, 1984.

[296] F. Corberi, G. Gonnella, E. Lippiello and M. Zannetti. Correlation functions and fluctuation-dissipation relation in driven mixtures: an exactly solvable model. Preprint, cond-mat/0210464

[297] L. Berthier, L. F. Cugliandolo and J. L. Iguain. Glassy systems under time-dependent driving forces: Application to slow granular rheology. Physical Review E, 63:051302/1-15, 2001.

[298] A. B. Kolton, R. Exartier, L. F. Cugliandolo, D. Dominguez and N. Gronbech-Jensen. Effective temperature in driven vortex lattices with random pinning. Preprint, cond-mat/0206042

[299] H. Horner. Drift, creep and pinning of a particle in a correlated random potential. Zeitschrift für Physik B, 100:243-258, 1996.

[300] F. Thalmann. Activated drift motion of a classical particle with a dynamical pinning effect. European Physical Journal B, 3:497-505, 1998.

[301] W. Kob and H. C. Andersen. Kinetic lattice-gas model of cage effects in high-density liquids and a test of mode-coupling theory of the ideal-glass transition. Physical Review E, 48:43644377, 1993.

[302] J. Kurchan, L. Peliti and M. Sellitto. Aging in lattice-gas models with constrained dynamics. Europhysics Letters, 39:365-370, 1997.

[303] M. Sellitto and J. J. Arenzon. Free-volume kinetic models of granular matter. Physical Review $E, 62: 7793-7796,2000$.

[304] M. Sellitto. Fluctuation-dissipation ratio in lattice-gas models with kinetic constraints. Acta Mechanica., 4:135-138, 1998.

[305] A. Barrat, J. Kurchan, V. Loreto and M. Sellitto. Edwards' measures: A thermodynamic construction for dense granular media and glasses. Physical Review E, 63:051301, 2001.

[306] A. Barrat, J. Kurchan, V. Loreto and M. Sellitto. Edwards' measures for powders and glasses. Physical Review Letters, 85:5034-5037, 2000.

[307] A. Barrat, V. Colizza and V. Loreto. Fluctuation-dissipation ratio for compacting granular media. Physical Review E, 66:011310, 2002.

[308] E. Caglioti, V. Loreto, H. J. Herrmann and M. Nicodemi. A "tetris-like" model for the compaction of dry granular media. Physical Review Letters, 79:15751578, 1997.

[309] M. Nicodemi. Dynamical response functions in models of vibrated granular media. Physical Review Letters, 82:3734-3737, 1999. 
[310] S. F. Edwards and D. V. Grinev. Statistical mechanics of vibration-induced compaction of powders. Physical Review E, 58:4758-62, 1998.

[311] S. F. Edwards and D. V. Grinev. Compactivity and transmission of stress in granular materials. Chaos, 82:5397-400, 1999.

[312] A. Lefevre and D. S. Dean. Tapping thermodynamics of the one-dimensional Ising model. Journal of Physics A, 34:L213-L220, 2001.

[313] H. A. Maakse and J. Kurchan. Testing the thermodynamic approach to granular matter with a numerical model of a decisive experiment. Nature, 415:614-617, 2002.

[314] Y. Levin, J. J. Arenzon and M. Sellitto. Aging dynamics and density relaxation in kinetic lattice gases under gravity. Europhysics Letters, 55:767-773, 2001.

[315] M. Sellitto. Effective temperature of an aging powder. Physical Review E, 63:060301(R), 2001.

[316] M. Sellitto. Effective temperature and compactivity of a lattice-gas under gravity. Physical Review E, 66:042101, 2002.

[317] A. Lefevre. Edwards measure and the steady state regime of a model with kinetic constraints under tapping. Journal of Physics A, 35:9037, 2002.

[318] S. Franz, R. Mulet and G. Parisi. Kob-andersen model: a nonstandard mechanism for the glassy transition. Physical Review E, 65:021506, 2002.

[319] G. H. Fredrickson and H. C. Andersen. Kinetic Ising-model of the glass-transition. Physical Review Letters, 53:1244-1247, 1984.

[320] G. H. Fredrickson and H. C. Andersen. Facilitated kinetic Ising-models and the glass-transition. Journal of Chemical Physics, 83:5822-5831, 1985.

[321] W. Kob and H. C. Andersen. Relaxation dynamics in a lattice gas: A test of the mode-coupling theory of the ideal glass transition. Physical Review E, 47:3281-3302, 1993.

[322] L. Peliti and M. Sellitto. Aging in a simple model of a structural glass. Journal de Physique (France), 8:49-56, 1998.

[323] L. Davison and D. Sherrington. Glassy behaviour in a simple topological model. Journal of Physics A, 33:8615-8625, 2000.

[324] L. Davison, D. Sherrington, J. P. Garrahan and A. Buhot. Glassy behaviour in a 3-state spin model. Journal of Physics A, 34:5147-5182, 2001.

[325] A. Lipowski and D. Johnston. Metastability in a four-spin Ising model. Journal of Physics A, 33:4451-4460, 2000.

[326] A. Lipowski and D. Johnston. Cooling-rate effects in a model of glasses. Physical Review E, 61:6375-6382, 2000.

[327] A. Buhot and J. P. Garrahan. Fluctuation-dissipation relations in the activated regime of simple strong-glass models. Physical Review Letters, 88:225702, 2002.

[328] J. Jackle and S. Eisinger. A hierarchically constrained kinetic ising-model. Zeitschrift für Physik B, 84:115-124, 1991.

[329] J. Reiter, F. Mauch and J. Jackle. Blocking transitions in lattice spin models with directed kinetic constraints. Physica A, 184:458-476, 1992.

[330] A. Buhot and J. P. Garrahan. Crossover from fragile to strong glassy behavior in kinetically constrained systems - art. no. 021505. Physical Review E, 6402:1505-+, 2001.

[331] F. Corberi, A. de Candia, E. Lippiello and M. Zannetti. Off equilibrium response function in the one dimensional random field ising model. Physical Review E, 65:046114, 2002.

[332] A. Crisanti, F. Ritort, A. Rocco and M. Sellitto. Is the stillinger and weber decomposition relevant for coarsening models? Journal of Physics-Condensed Matter, 14:1523-1538, 2002.

[333] M. E. J. Newman and C. Moore. Glassy dynamics and aging in an exactly solvable spin model. Physical Review E, 60:5068-5072, 1999.

[334] J. P. Garrahan and M. E. J. Newman. Glassiness and constrained dynamics of a short-range nondisordered spin model. Physical Review E, 62:7670-7678, 2000.

[335] G.B. Alers, M.B. Weissmann and N.E. Israeloff. Mesoscopic tests for thermally chaotic states in a cumn spin glass. Physical Review B, 46:507509, 1992.

[336] M. B. Weissman. What is a spin glass? a glimpse via mesoscopic noise. Review of Modern Physics, 65:829-839, 1993.

[337] M. Alba, J. Hammann, M. Ocio, P. Refregier and H. Bouchiat. Spin-glass dynamics from magnetic noise, relaxation, and susceptibility measurements. Journal Applied Physics, 61:3683-8, 1987.

[338] P. Refregier and M. Ocio. Measurement of spontaneous magnetic fluctuations. Revue de Physique Appliquee, 22:367-74, 1987.

[339] H. Bouchiat. Experimental studies of the spin glass dynamics: towards a better understanding of the spatial correlations in the spin glass phase? Physica A, 163:284-290, 1990.

[340] D. Herisson and M. Ocio. Fluctuation-dissipation ratio of a spin glass in the aging regime. 
Physical Review Letters, 88:257202, 2002.

[341] L. F. Cugliandolo, D. R. Grempel , J. Kurchan and E. Vincent. A search for fluctuationdissipation theorem violations in spin-glasses from susceptibility data. Europhysics Letters, 48:699-705, 1999.

[342] T. S. Grigera and N. E. Israeloff. Observation of fluctuation-dissipation-theorem violations in a structural glass. Physical Review Letters, 83:5038, 1999.

[343] L. Bellon, S. Ciliberto and C. Laroche. Violation of the fluctuation-dissipation relation during the formation of a colloidal glass. Europhysics Letters, 53:511-17, 2001.

[344] L. Bellon and S. Ciliberto. Experimental study of the fluctuation-dissipation-relation during an aging process. Preprint, cond-mat/0201224 